SPATIAL AND TEMPORAL GENETIC STRUCTURE OF THE

NEW ZEALAND SCALLOP Pecten novaezelandiae:

\title{
A MULTIDISCIPLINARY PERSPECTIVE
}

BY

CATARINA NUNES SOARES SILVA

A thesis submitted to the Victoria University of Wellington

in fulfilment of the requirements for the degree of

Doctor of Philosophy

Victoria University of Wellington

Te Whare Wānanga o te Ūpoko o te Ika a Māui

2015 
This thesis was conducted under the supervision of

\author{
Prof. Jonathan Gardner
}

$\&$

A. Prof. James Bell

Victoria University of Wellington

Wellington, New Zealand

TE WHARE WĀNANGA O TE ŪPOKO O TE IKA A MĀUI

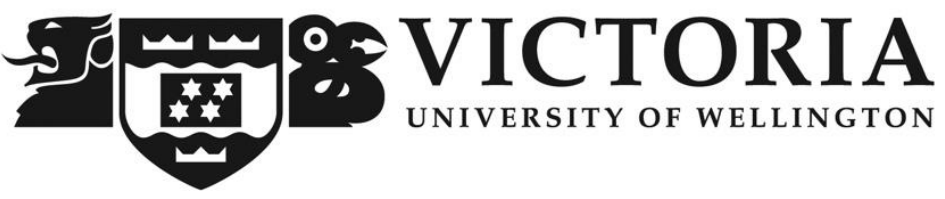




\section{Abstract}

Knowledge about the population genetic structure of species and the factors shaping such patterns is crucial for effective management and conservation. The complexity of New Zealand's marine environment presents a challenge for management and the classification of its marine biogeographic areas. As such, it is an interesting system to investigate marine connectivity dynamics and the evolutionary processes shaping the population structure of marine species. An accurate description of spatial and temporal patterns of dispersal and population structure requires the use of tools capable of incorporating the variability of the mechanisms involved. However, these techniques are yet to be broadly applied to New Zealand marine organisms.

This study used genetic markers to assess the genetic variation of the endemic New Zealand scallop, Pecten novaezelandiae, at different spatial and temporal scales. A multidisciplinary approach was used integrating genetic with environmental data (seascape genetics) and hydrodynamic modelling tools. $P$. novaezelandiae supports important commercial, recreational and customary fisheries but there is no previous information about its genetic structure. Therefore, twelve microsatellite markers were developed for this study (Chapter 2).

Samples $(n=952)$ were collected from 15 locations to determine the genetic structure across the distribution range of $P$. novaezelandiae. The low genetic structure detected in this study is expected given the recent evolutionary history, the large reproductive potential and the pelagic larval duration of the species (approximately 3 weeks). A significant isolation by distance signal and a degree of differentiation from north to south was apparent, but this structure conflicted with some evidence of panmixia. A latitudinal genetic diversity gradient was observed that might reflect the colonisation and extinction events and insufficient time to reach migration-drift equilibrium during a recent range expansion (Chapter 3).

A seascape genetic approach was used to test for associations between patterns of genetic variation in $P$. novaezelandiae and environmental variables (three geospatial and six environmental variables). Although the geographic distance between populations was an important variable explaining the genetic variation among populations, it appears that levels of genetic differentiation are not a simple function of distance. Evidence suggests 
that some environmental factors such as freshwater discharge and suspended particulate matter can be contributing to the patterns of genetic differentiation of $P$. novaezelandiae in New Zealand (Chapter 4).

Dispersal of $P$. novaezelandiae was investigated at a small spatial and temporal scale in the Coromandel fishery using genetic markers integrated with hydrodynamic modelling. For the spatial analysis, samples $(n=402)$ were collected in 2012 from 5 locations and for the temporal analysis samples $(n=383)$ were collected in 2012 and 2014 from 3 locations. Results showed small but significant spatial and temporal genetic differentiation, suggesting that the Coromandel fishery does not form a single panmictic unit with free gene flow and supporting a model of source-sink population dynamics (Chapter 5).

The importance of using multidisciplinary approaches at different spatial and temporal scales is widely recognized as a means to better understand the complex processes affecting marine connectivity. The outcomes of this study highlight the importance of incorporating these different approaches, provide vital information to assist in effective management and conservation of $P$. novaezelandiae and contribute to our understanding of evolutionary processes shaping population structure of marine species. 


\section{Acknowledgments}

I would like to thank my primary supervisor Professor Jonathan Gardner for the opportunity to do this research in such a beautiful corner of the world and for his contribution to the work contained herein. I also greatly appreciate the support of my secondary supervisor Associate Professor James Bell shortly after my arrival and his helpful feedback on the content of this thesis. Thank you to the members of my committee Dr. Peter Ritchie, Dr. Shane Lavery, Associate Professor Joe Zuccarello and Dr. Martin Cryer (MPI) for the valuable advice and input in the early stages of my studies.

This research would not have been possible without the enormous generosity of everyone who helped with sample collection. I would like to thank Dr. James Williams (NIWA) for all the support and for connecting me with fishermen for sample collection (Karl Aislabie from Scallop Coromandel Fishery and Donna and Ewan MacMillan from Sodablast Northland ltd). Thank you to Daniel McNaughtan and Paul Mensink for assisting and collecting scallops from Wellington Harbour. Dr. Shane Geange kindly collected scallops from Fiordland, Dr. Debbie Freeman and Brenton Twist provided tissue of scallops from Stewart Is (thanks to Dr. Chris Hepburn for putting me in contact with Brenton), David Middleton and Oliver Wilson provided scallops from Coromandel and Reid Forrest provided scallops from the Marlborough sounds. I would also like to thank Dr. Shane Lavery for putting me in contact with students and staff during my time at Auckland University; thanks to Paul Caiger for helping with sample collection and everyone at Auckland University who welcomed me so warmly. I would also like to thanks Dr. Matt Pinkerton for generating the variables from the ocean colour satellite data MODIS project.

I would like to thank my lab mates for sharing their knowledge with me and for their friendship, especially Danielle Hannan and Heather Constable for all the valuable advice and support in the beginning of this research. I would also like to thank the staff from the School of Biological Sciences and the Faculty of Sciences, specially Mary Murray, Sandra Taylor, Patricia Stein, Paul Marsden and Mark Stephen for all the logistical support and infinite patience. I am also thankful for the helpful feedback that Dr. Simon Davy, Dr. Ian Hogg and Dr. Jennifer Ovenden gave on my thesis and for the time and effort they spent in the examination process. 
The Ministry for Primary Industries provided funding for this research (Biodiversity Research Advisory Group project ZBD2009-10), which gave me the privilege to work on this research. I also received considerable funding from Victoria University of Wellington; I am grateful for the Victoria Doctoral Scholarship, Victoria PhD Submission Scholarship, Victoria University of Wellington Students Association Sponsorship, VUW Faculty of Science Strategic Research Grant and Kathleen Stewart Postgraduate Scholarship that allowed me to pursue my studies and to attend international conferences.

I am thankful for the opportunity to volunteer as Vice-President of the Society for Conservation Biology VUW Chapter. Thanks to the committee and other members for all the valuable knowledge and for our stimulating discussions. I am very grateful for the financial support of the Society for Conservation Biology to attend international conferences.

This journey would not have been so rewarding without the support of my Wellingtonian family. I am very grateful to have made such good friends, thank you all for the good moments. I would also like to thank many good friends overseas for their support and their special sense of humour.

Last but not least, a very special thank you to my parents, Madalena and António, who always encouraged my decisions and fuelled my passion for biology and to my partner João for all the support and for ensuring my healthy levels of insanity. 
"One planet, one experiment."

E. O. Wilson 


\section{Table of contents}

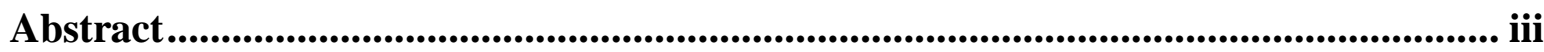

Acknowledgments ...............................................................................................................................v

Table of contents............................................................................................................................. viii

List of Figures ………….......................................................................................................................

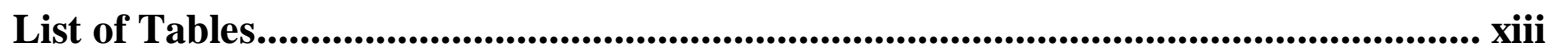

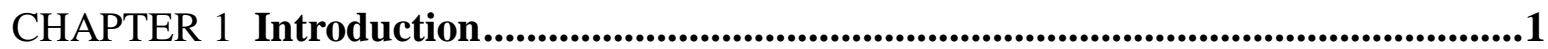

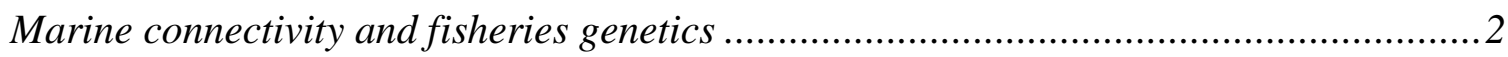

Environmental factors affecting genetic variation ......................................................6

The New Zealand marine environment ....................................................................

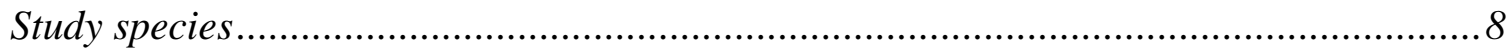

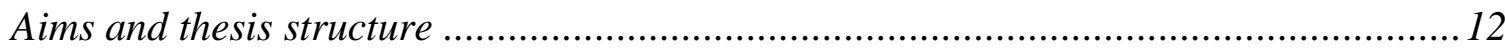

CHAPTER 2 Development of microsatellite DNA markers for the New Zealand scallop Pecten novaezelandiae ....................................................................................................15

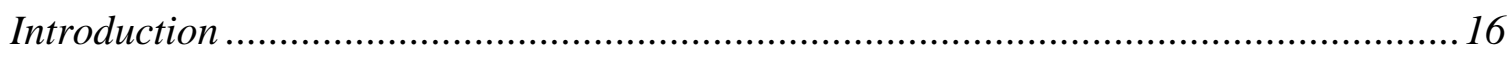

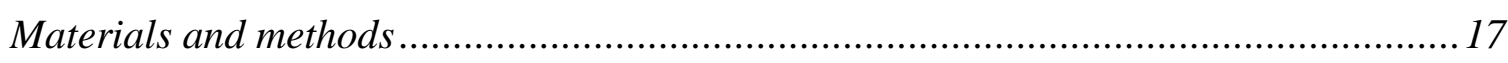

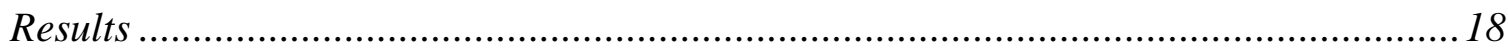

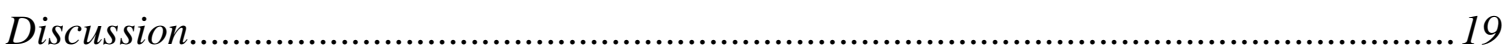

CHAPTER 3 Genetic structure of Pecten novaezelandiae throughout its distribution range...............................................................................................................................23

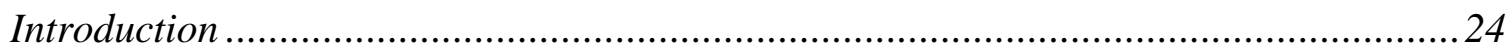

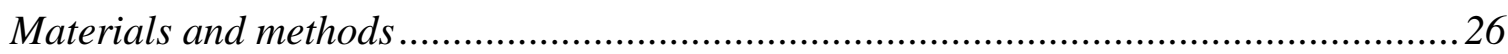

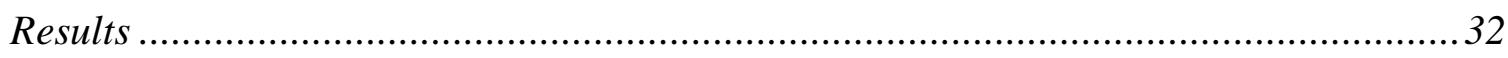

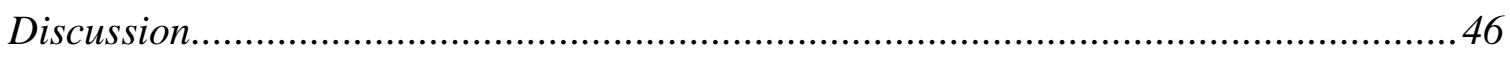


CHAPTER 4 Seascape genetics of Pecten novaezelandiae: linking environmental and genetic variation.............................................................................................................53

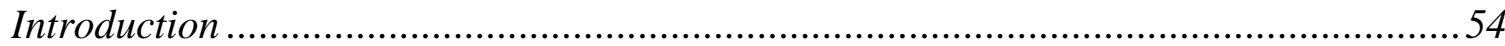

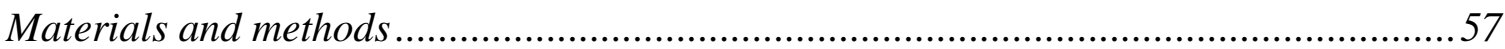

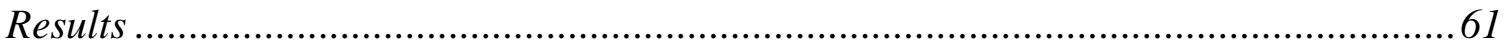

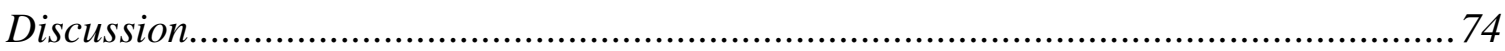

CHAPTER 5 Spatial and temporal patterns of dispersal of Pecten novaezelandiae in the Coromandel fishery: implications for management ............................................81

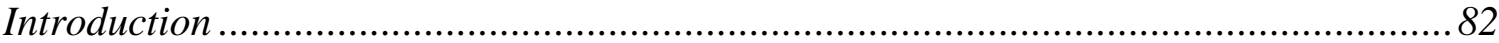

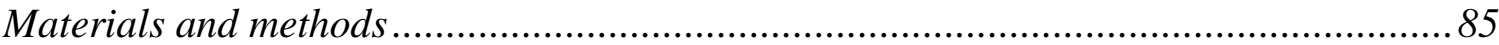

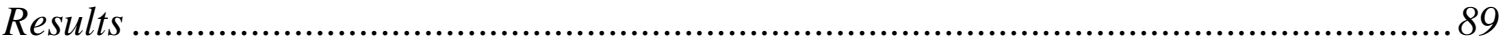

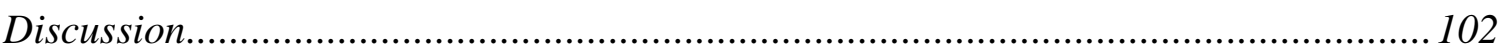

CHAPTER 6 General discussion .............................................................................109

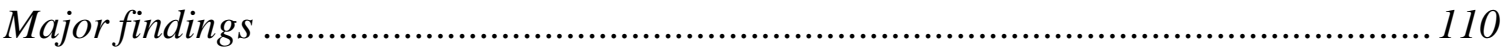

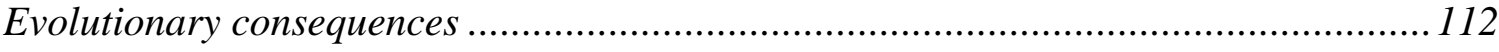

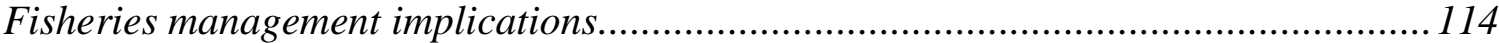

Contribution to the field and future directions ..................................................... 117

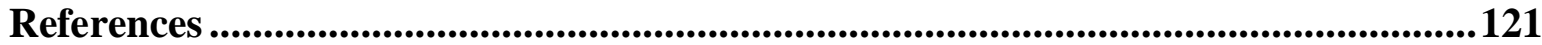

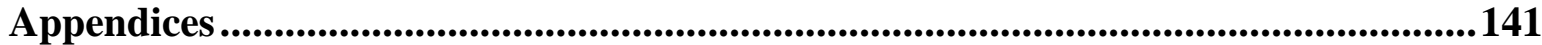




\section{List of Figures}

Figure 1.1 Diagrammatic representation of the life cycle of Pecten maximus (Pennec et al. 2003)

Figure 1.2 Map of the location of quota management areas (QMAs) used for management of the commercial and recreational fishery of Pecten novaezelandiae (sourced from www.nabis.govt.nz)

Figure 2.1 Length frequency of the 88199,8597 and 218608 reads, respectively, obtained with a 1/8th run on a 454 GS FLX instrument for Pecten novaezelandiae. 19

Figure 3.1 Map of sampling sites of Pecten novaezelandiae collected from New Zealand. Abbreviations as per Table 3.1

Figure 3.2 Size structure of Pecten novaezelandiae from 14 study sites in New Zealand (shells from samples collected in STE12 were not available). Dashed lines represent the maximum and minimum size at each site, while the bars represent the 25 th and 75th quartile of size ranges, with the median indicated within each bar. 32

Figure 3.3 Principal components analysis (PCoA) for Pecten novaezelandiae using pairwise genetic distance based on 10 microsatellite loci. 38

Figure 3.4 Neighbor joining population tree for Pecten novaezelandiae based on $F_{\mathrm{ST}}$ values using 14 populations.

Figure 3.5 AWclust results for Pecten novaezelandiae using 10 loci: expected (E) and observed $(\mathrm{O}) \log$ pooled with-in cluster sum of squares (Wk) (on the left) and gap statistic values between the observed and expected (on the right).

Figure 3.6 Bayesian cluster analysis (Structure) results for Pecten novaezelandiae using 10 loci $(\mathrm{K}=2)$. Samples were assigned to two colour coded-genetic clusters (blue and red).

Figure 3.7 Spatial Bayesian analysis (Geneland) results for Pecten novaezelandiae using 10 loci: (a) posterior density distribution of the number of clusters $(k=5)$, (b) map of population membership for $\mathrm{k}=5$ 
Figure 3.8 Spatial Bayesian analysis (Geneland) results for Pecten novaezelandiae using 10 loci: (a-e) assignment of individuals to clusters for $\mathrm{K}=1$ to $\mathrm{K}=5$, respectively. The highest population membership values are in light yellow and level curves indicate spatial changes in assignment values.

Figure 3.9 Scatterplot of Mantel tests using $F_{\mathrm{ST}}$ for all the mainland populations $\left(\mathrm{R}^{2}=0.324\right.$, $\mathrm{p}<0.01)$, when excluding STE12 $(\mathrm{n}=10) \mathrm{R}^{2}=0.461(\mathrm{p}<0.01)$ and when just the north and central groups were included $\mathrm{R}^{2}=0.148$ ( $\left.\mathrm{p}=0.05\right)$. STE14: Stewart Is, Port Pegasus; FIO: Fiordland; STE12: Stewart Is, Paterson Inlet; North Central Groups: remaining populations.

Figure 3.10 Linear regression analyses of allelic richness, $A_{R}\left(R^{2}=0.688, p=0.0001\right)$ and private allelic richness, $\mathrm{PA}_{\mathrm{R}}\left(\mathrm{R}^{2}=0.683, \mathrm{p}=0.0002\right)$ as a function of latitude. 45

Figure 4.1 Map of sampling sites of Pecten novaezelandiae collected from New Zealand. Locations abbreviations as per Table 3.1. .58

Figure 4.2 Principal component analysis (PCA) of both the geospatial and environmental variables. Abbreviations as per Table 4.1.

Figure 5.1 Map of sampling sites in the Coromandel region: (a) for the spatial analyses individuals of Pecten novaezelandiae were collected in 2012 and (b) for the temporal analyses a subset of individuals collected in 2012 and additional samples collected in 2014 was used. Locations abbreviations as per Table 5.1. 85

Figure 5.2 Size structure of individuals of Pecten novaezelandiae collected in the Coromandel region for the (a) spatial and (b) temporal studies. Dashed lines represent the maximum and minimum size at each site, while the bars represent the 25th and 75 th quartile of size ranges, with the median indicated within each bar. Location abbreviations as per Table 5.1

Figure 5.3 Length-frequency histograms of Pecten novaezelandiae collected in 2012 and 2014 in the Coromandel region for the temporal analyses. Location abbreviations as per Table 5.1. 90

Figure 5.4 Neighbour joining population tree for Pecten novaezelandiae based on $\mathrm{F}_{\mathrm{ST}}$ values for (a) the spatial study and (b) the temporal study. Location abbreviations as per Table 5.1. 
Figure 5.5 Principal components analysis (PCoA) for Pecten novaezelandiae using pairwise genetic distance for (a) the spatial and (b) the temporal study. Locations abbreviations as per Table 5.1 .

Figure 5.6 AWclust analyses for Pecten novaezelandiae for (a) the spatial study and (b) the temporal study.

Figure 5.7 AWclust analysis results for Pecten novaezelandiae for the spatial study. Each colour represents an estimated portion of $\mathrm{K}=3$ clusters. Locations abbreviations as per Table 5.1 .95

Figure 5.8 AWclust analysis results for Pecten novaezelandiae for the temporal study. Each colour represents an estimated portion of $\mathrm{K}=6$ clusters. Locations abbreviations as per Table 5.1 .95

Figure 5.9 Mean values of observed relatedness for Pecten novaezelandiae for the temporal study. Upper and lower error bars represent the 95\% confidence interval about the mean values as determined by 1000 bootstraps resampling. Upper (U) and lower (L) confidence limits represent the $95 \%$ confidence interval for the null hypothesis of 'No Difference' across the locations as determined by 999 permutations. Location abbreviations as per Table 5.1. 101

Figure 5.10 Probability distributions of particles released at (a) Little Barrier Island (LBI), (b) Mercury Island (MER) and (c) Tauranga (TAU). Maps show results of particles with diel vertical migration run for 25 days and released in early summer. Results are averaged across ten years (1997-2007). The colour bar indicates final position probability (\%). Location abbreviations as per Table 5.1. 102

Figure 5.11 Expected relationships between locations sampled in Coromandel with estimated levels of larval dispersal and self-recruitment (low, moderate and high) indicated by the arrows. Circles are locations sampled, site abbreviations as per Table 5.1 .

Supplementary Figure 1 Allele discovery curves for Pecten novaezelandiae (Chapter 3).142 


\section{List of Tables}

Table 2.1 Polymorphic microsatellite markers for $P$. novaezelandiae with locus characteristics, number of alleles $\left(\mathrm{N}_{\mathrm{a}}\right)$, observed heterozygosity $\left(\mathrm{H}_{\mathrm{o}}\right)$, expected heterozygosity $\left(\mathrm{H}_{\mathrm{e}}\right)$, probability of being out of Hardy-Weinberg Equilibrium (HWE).

Table 3.1 Pecten novaezelandiae collecting site descriptions, sample sizes $(\mathrm{N})$, mean depth $(\mathrm{m})$, dates and geospatial coordinates

Table 3.2 Descriptive measures of genetic variation in $P$. novaezelandiae for 10 loci. Allelic richness $\left(A_{R}\right)$, private allelic richness $\left(P_{R}\right)$, number of private alleles per site $(\mathrm{Pa})$, observed heterozygosity $\left(\mathrm{H}_{\mathrm{O}}\right)$, expected heterozygosity $\left(\mathrm{H}_{\mathrm{E}}\right)$ and fixation index $\left(\mathrm{F}_{\mathrm{IS}}\right)$

Table 3.3 Pairwise $F_{\mathrm{ST}}$ values for Pecten novaezelandiae using 10 loci are below the diagonal and $\mathrm{R}_{\mathrm{ST}}$ values are above the diagonal. Significant values are in bold after FDR testing. Mean $F_{\mathrm{ST}}$ values for each population are in the bottom row 36

Table $3.4 F^{\prime}$ ST values for Pecten novaezelandiae using 10 loci below diagonal and $\mathrm{D}_{\mathrm{ST}}$ values above diagonal. 37

Table 3.5 Analysis of molecular variance (AMOVA) for 14 populations from 4 regions using 10 loci.

Table 3.6 Evanno table (Evanno et al. 2005) output for 14 populations from 4 regions of Pecten novaezelandiae.

Table 3.7 Number of first generation (F0) migrants detected for Pecten novaezelandiae at each sampled region.

Table 3.8 Percentage of individuals of Pecten novaezelandiae collected from each sampling region, assigned to each sampled potential source region. "Total other regions" is the percentage of individuals recruited from regions other than the collection site. 46

Table 4.1 Environmental variables (name), abbreviation, description and units from (a) the Marine Environment Classification (MEC), (b) the Benthic-optimised Marine Environment Classification (BOMEC) and (c) data obtained from the MODIS project. 
Table 4.2 Correlations between variables as defined by Pearson's correlation test. Significantly correlated variables at $\mathrm{p}<0.05$ are in bold. Abbreviations as per Table 4.1 . 62

Table 4.3 Results of the top ten best fitting models of generalized linear model analyses (GLZ routine in STATISTICA v12) testing for the contribution of all 9 geospatial and environmental variables to explain variation in $F_{\mathrm{ST}}$ and $F_{\text {ST }}^{\prime}$ for Pecten novaezelandiae. The checkmarks $(\checkmark)$ indicate which variables were included in each model. ${ }^{1}$ p-value of each model; ${ }^{2} \mathrm{p}$-value of the test of all effects, significant values at $\mathrm{p}<0.05$ are in bold.

Table 4.4 Results of the top ten best fitting models of generalized linear model analyses (GLZ routine in STATISTICA v12) testing for the contribution of 3 geospatial variables to explain variation in $F_{\mathrm{ST}}$ and $F_{\mathrm{ST}}^{\prime}$ for Pecten novaezelandiae. The checkmarks $(\checkmark)$ indicate which variables were included in each model. ${ }^{1}$ p-value of each model; ${ }^{2}$ p-value of the test of all effects.

Table 4.5 Results of the top ten best fitting models of generalized linear model analyses (GLZ routine in STATISTICA v12) testing for the contribution of 6 environmental variables to explain variation in $F_{\mathrm{ST}}$ and $F_{\text {ST }}^{\prime}$ for Pecten novaezelandiae. The checkmarks $(\checkmark)$ indicate which variables were included in each model. ${ }^{1} \mathrm{p}$-value of each model; ${ }^{2} \mathrm{p}$-value of the test of all effects, significant values at $\mathrm{p}<0.05$ are in bold.

Table 4.6 Results of the ten best fitting models with respective Spearman's Rho $\left(\mathrm{r}_{\mathrm{s}}\right)$ values from the BEST analyses testing for the contribution of all 9 geospatial and environmental variables to explain variation in allele frequencies for Pecten novaezelandiae. The checkmarks $(\checkmark)$ indicate which variables were included in each model. 70

Table 4.7 Results of the ten best fitting models with respective Spearman's Rho $\left(\mathrm{r}_{\mathrm{s}}\right)$ values from the BEST analyses testing for the contribution of 3 geospatial variables to explain variation in allele frequencies for Pecten novaezelandiae. The checkmarks $(\checkmark)$ indicate which variables were included in each model.

Table 4.8 Results of the ten best fitting models with respective Spearman's Rho $\left(\mathrm{r}_{\mathrm{s}}\right)$ values from the BEST analyses testing for the contribution of 6 environmental variables to 
explain variation in allele frequencies for Pecten novaezelandiae. The checkmarks $(\checkmark)$ indicate which variables were included in each model.

Table 4.9 Results of the BEST analyses testing for the contribution of 9 geospatial and environmental variables to explain variation in allele frequencies for each locus for Pecten novaezelandiae for all 14 locations. Top part of table represents the best fitting model for each locus; the checkmarks $(\checkmark)$ indicate which variables were included in the model. Bottom part of table indicates the number of times that each variable was included in the top ten best fitting models. 73

Table 4.10 Summary of GLZ and BEST results with important variables explaining genetic variation as determined by the number of times it was included in the models, model $p$-values, test of all effects $p$-values and significance of $r_{s}$ coefficients. .74

Table 5.1 Collecting site descriptions for the (a) spatial and (b) temporal analyses of Pecten novaezelandiae in the Coromandel region with sample sizes $(\mathrm{N})$, mean depth $(\mathrm{m})$, dates and geospatial coordinates.

Table 5.2 Genetic variation in P. novaezelandiae in the Coromandel region for locations used for (a) the spatial study and (b) the temporal study. Allelic richness $\left(A_{R}\right)$, private allelic richness $\left(P A_{R}\right)$, number of private alleles per site $(\mathrm{Pa})$, observed heterozygosity $\left(\mathrm{H}_{\mathrm{O}}\right)$, expected heterozygosity $\left(\mathrm{H}_{\mathrm{E}}\right)$ and fixation index $\left(\mathrm{F}_{\mathrm{IS}}\right)$. $\mathrm{F}_{\mathrm{IS}}$ values in bold show significant departure from HWE expectations after FDR correction $(p<0.05)$. Locations abbreviations as per Table 5.1.

Table 5.3 Pairwise F $_{\mathrm{ST}}$ values for Pecten novaezelandiae for the spatial study are below the diagonal and p-values are above the diagonal. Significant values are in bold after FDR testing $(\mathrm{p}<0.05)$. Locations abbreviations as per Table 5.1.

Table 5.4 Pairwise FST values for Pecten novaezelandiae for the temporal study are below the diagonal and p-values are above the diagonal. Significant values are in bold after FDR testing $(\mathrm{p}<0.05)$. Locations abbreviations as per Table 5.1. 92

Table 5.5 Evanno table (Evanno et al. 2005) output for (a) the spatial study and (b) the temporal study of genetic structure of Pecten novaezelandiae. 96

Table 5.6 Analysis of molecular variance (AMOVA) for the spatial study of $P$. novaezelandiae using 3 regions. 
Table 5.7 Analysis of molecular variance (AMOVA) for the spatial study of $P$. novaezelandiae using 4 regions.

Table 5.8 Analysis of molecular variance (AMOVA) for the temporal study of $P$. novaezelandiae using all locations grouped in 2 regions (between years). 98

Table 5.9 Analysis of molecular variance (AMOVA) for the temporal study of $P$. novaezelandiae using locations MER and TAU grouped in 2 regions (between years).

Table 5.10 Number of first generation (F0) migrants detected for Pecten novaezelandiae at each sampled location for the spatial study in Coromandel. Locations abbreviations as per Table 5.1.

Table 5.11 Percentage of individuals of Pecten novaezelandiae collected from each sampling location, assigned to each sampled potential source location for the spatial study in the Coromandel. "Total other regions" is the percentage of individuals recruited from regions other than the collection site. Location abbreviations as per Table 5.1

Table 5.12 Number of first generation (F0) migrants detected for Pecten novaezelandiae at each sampled location for the temporal study in the Coromandel. Location abbreviations as per Table 5.1. 100

Table 5.13 Percentage of individuals of Pecten novaezelandiae collected from each sampling location, assigned to each sampled potential source location for the temporal study in the Coromandel. "Total other regions" is the percentage of individuals recruited from regions other than the collection site. Location abbreviations as per Table 5.1

Supplementary Table 1 PCR conditions for 12 microsatellite loci of Pecten novaezelandiae (Chapter 2). 141

Supplementary Table 2 Allele frequencies for 10 microsatellite loci of Pecten novaezelandiae for 15 locations (Chapter 3). 144

Supplementary Table 3 Allele frequencies for 10 microsatellite loci of Pecten novaezelandiae for 5 locations in the Coromandel fishery (Chapter 5 - spatial study). 
Supplementary Table 4 Allele frequencies for 10 microsatellite loci of Pecten novaezelandiae for 6 locations in the Coromandel fishery (Chapter 5 -temporal

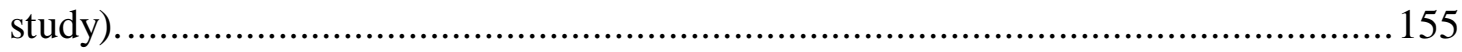



CHAPTER 1

Introduction 


\section{Marine connectivity and fisheries genetics}

Most marine invertebrates have a planktonic larval stage of development (Strathmann et al. 2002). This period in the water column is typically the larval dispersal phase of the species and greatly determines population connectivity and patterns of population structure of species. Population connectivity can be defined as the exchange of individuals among geographically separated subpopulations (Cowen et al. 2007), with connectivity also being influenced by post-settlement mortality, growth, and fitness from settlement to successful reproduction (Pineda et al. 2007). Population connectivity is therefore an important process for population replenishment and the management of marine ecosystems. Understanding the patterns of genetic structure of marine species, and the processes driving such patterns, is essential not only for learning about the ecology and evolution of the species but also for implementing effective management strategies. To identify the most likely source populations, i.e. populations with larvae contributing to local recruitment, is particularly important in fisheries since it influences population growth in fished areas (Soria et al. 2012). Moreover, biologically distinctive populations play a key role in providing resilience and in assuring fisheries productivity (Aguirre \& Marshall 2012). This is particularly important for organisms with large periodic population fluctuations and highly variable recruitment, such as most scallop species (Shumway \& Parsons 2006).

Scallops support important fisheries around the world. More than 400 species are known to occur from the tropics to the poles and over 30 species are commercially important. King scallops (Pecten spp.) are the most commercially exploited species and are found mostly at high latitudes in both hemispheres, along the coasts of Europe, Africa, Asia, Australia, New Zealand and their neighbouring islands (Brand, 2006 in Shumway \& Parsons, 2006). Because of the general interest in scallops associated with their high economic value, they have been subject of considerable research effort. Studies on population genetic structure have been conducted for Aequipecten opercularis (Arias et al. 2010), Argopecten irradians irradians (Bricelj \& Krause 1992), Chlamys farreri (Zhan et al. 2009), Chlamys opercularis (Macleod et al. 1985), Nodipecten subnodosus (Petersen et al. 2010), Patinopecten yessoensis (Nagashima et al. 2005; Sato et al. 2005; Chen et al. 2009), Pecten fumatus (Woodburn 1990), Pecten jacobaeus (Rios et al. 2002), Pecten maximus (Wilding et al. 1997; Heipel et al. 1998; Rios et al. 2002) and Placopecten magellanicus (e.g. Kenchington et al. 2006). However, in New Zealand, the population genetic structure of the 
endemic Pecten novaezelandiae Reeve, 1852 is currently unknown. As observed in other species, the New Zealand scallop has highly variable recruitment (Shumway \& Parsons 2006) and the processes that result in large periodic population fluctuations are poorly understood; therefore fisheries managers are confronted with uncertainty during the decision-making process.

Typically, fisheries are divided into management units or stocks, which can be homogeneous groups regarding, for example, growth and mortality rates. There are several stock definitions (reviewed in Carvalho and Hauser 1994), but in general, a stock can be defined as a group of individuals sharing similar vital rates such as maturity, growth and mortality and with a closed life cycle in which recruits are originated by previous generations in the same group (Cadrin et al. 2005). In practice, the application of the stock definition is very complex. Populations are rarely differentiated by evident boundaries and in marine organisms this categorisation is even more complex when compared to terrestrial organisms. This is because marine organisms are commonly expected to show lower geographical differentiation. Such an assumption is the result of, for example, less physical barriers observed in an "open" ocean and the planktonic larval phases responsible for the dispersal of some marine species. Nevertheless, recent studies have highlighted the complexity of marine population dynamics and geographical differentiation across many species (e.g. Apte and Gardner 2002; Kenchington et al. 2006; Zhan et al. 2009).

An understanding of population genetic structure was incorporated in stock definition in the late twentieth-century. Larkin (1972) described a stock as "... a population of organisms which, sharing a common gene pool, is sufficiently discrete to warrant consideration as a self-perpetuating system which can be managed". In the past three decades a great variety of studies in the field of fisheries genetics have demonstrated that integrating genetic information with traditional methods of fishery stock assessment can extensively improve the quality of management recommendations (e.g. Sweijd et al. 2000; Ward 2000; Hauser \& Carvalho 2008; Saillant et al. 2012). As a consequence, the importance of genetic diversity at the population level for assuring adaptive responses to natural modifications as well as environmental changes caused by man is now broadly recognized (Bonin et al. 2007; Aguirre \& Marshall 2012; Allendorf et al. 2014).

Several different approaches have been used to identify stocks and investigate population structure and connectivity. Direct methods, such as tagging, have been 
extremely useful for defining the movement of fish species (McFarlane et al. 1990). However, invertebrate species with a dispersal period as a pelagic larva are logistically very difficult to track due to the small sizes of larvae. The use of molecular markers can provide valuable information on the dispersal movement among populations or stocks, on the reproductive success of individuals and can also provide genetic information over previous generations (Selkoe \& Toonen 2006). Consequently, they allow for the assessment of variability between stocks and a better understanding of the replenishment of fisheries (Carvalho \& Hauser 1994).

Different genetic markers have been used for the assessment of population genetic structure and connectivity. For example, in the 1960s, protein allozymes were the first markers used to address genetic variation within individuals. However, as allozymes are proteins encoded by DNA and not all variation in DNA sequence results in different proteins, allozyme studies did not capture all the information contained in the DNA. It tended to underestimate levels of genetic variability and caused constraints on sample collection and storage (Carvalho 1998). With the technical advance of molecular biology, several types of molecular markers have become prevalent in ecological studies. Mitochondrial DNA (mtDNA) markers have been used extensively mainly because they are relatively easy to amplify without previous information on the DNA sequence, mutation rates are reasonably high, in most animals mtDNA is inherited maternally which makes individual lineages easy to track and because there are few copies of mtDNA (it is effectively a quarter of the size of diploid nuclear DNA) it is reasonably sensitive to demographic events. Nevertheless, the use of mtDNA has some limitations as it may not be representative of the whole population and the high probability of haplotype losses may underestimate the levels of genetic diversity (Park \& Moran 1994). Another method that has been used since the 1990s is the PCR-based method named random amplified polymorphic DNA (RAPDs). Although RAPDs can be a relatively quick method, amplification of bands frequently varies and bands that are amplified from non-target DNA can be difficult to detect. Another method also used is the amplified fragment length polymorphism (AFLPs), which is more reliable than RAPDs but it is also more labour intensive. Finally, single nucleotide polymorphisms (SNPs) are becoming increasingly more popular for population genetic structure studies and although their development and use is relatively recent and expensive they hold great potential for a wide range of applications (Freeland 2005). 
As a result of being easily influenced by migration, rapidly developed and because of their decreasing cost of development, microsatellites are currently the most popular and versatile DNA markers for ecological applications; their utility and limitations are well known (Freeland 2005; Selkoe \& Toonen 2006). Microsatellites are short sequences of DNA consisting of tandem repeats of 2-10 bp. They are highly polymorphic (i.e., multiple alleles at each locus due to high rates of mutation), co-dominant (i.e., homozygous and heterozygous individuals can be identified, meaning that allele frequencies are easily calculated), usually species-specific, commonly considered as selectivity neutral and broadly distributed in the genome (Freeland 2005). Microsatellites are regarded as high resolution genetic markers and can reveal very high genetic diversity at fine geographical scales (e.g. Zhan et al. 2009). Microsatellites have high mutation rates compared to other types of sequences. Although these mutations can be highly variable, microsatellite estimated mutation rates, as for example $5.56 \times 10^{-4}$ in the common carp (Cyprinus carpio) (Yue et al., 2007), are considerably higher than the estimated overall point mutation rate of nuclear DNA, which is approximately $10^{-9}$ to $10^{-10}(\mathrm{Li} 1997)$. The high number of mutations in microsatellites is usually attributed to slipped-strand mis-pairing that occurs during DNA replication. This characteristic makes microsatellite data particularly appropriate for deducing comparatively recent population genetic events (Freeland et al., 2011). Microsatellite markers had been developed for many scallop species (e.g. Sato et al. 2005; Watts et al. 2005; Ma \& Yu 2009; Arias et al. 2010; Charrier et al. 2012; Marín et al. 2012) and have shown high variability, suggesting that they are suitable for being used in the family Pectinidae.

Population genetics is a powerful tool to address marine connectivity and population dynamics. Although random genetic drift, gene flow, mutation and selection are the main forces shaping the patterns of genetic structure, stochastic processes add an inevitable uncertainty in the recruitment of organisms (Siegel et al. 2008). Indirect techniques such as the use of molecular markers have been widely used to understand dispersal patterns and marine connectivity (Hellberg et al. 2002). However, the integrated use of molecular markers with other methods is recognized as a better approach to help clarify the complex dynamics of marine populations (Cowen \& Sponaugle 2009). Multidisciplinary approaches combining genetic and ecological information are particularly valuable when genetic differentiation is low, which is the case for many marine species (Selkoe et al. 2008). 


\section{Environmental factors affecting genetic variation}

Population genetic structure of organisms can be strongly influenced by biological factors such as the pelagic larval duration of the species and also by current and historical environmental features. For example, it is commonly recognized that connectivity among marine populations is strongly influenced by coastal currents (Ross et al. 2009; White et al. 2010). Understanding the effects that environmental variation has on population genetic structure is a central topic for management and conservation. Consequently, approaches that integrate biological characteristics, spatial structure and oceanographic features are valuable tools for understanding connectivity among populations (Selkoe et al. 2010).

Landscape genetics is a discipline that analyses the influence of landscape characteristics on genetic structure of populations (Manel et al. 2003). In the aquatic environment it is often named seascape genetics and aims to understand the factors (e.g. ocean currents) that shape gene flow dynamics; however, compared to landscape genetics, studies applying this concept in the marine environment are still scarce (but see Galindo et al. 2006; White et al. 2010; Schunter et al. 2011; Wei et al. 2013b; Constable 2014; Hannan 2014) and in only one recent study with the rock scallop Spondylus calcifer (Soria et al. 2012).

Seascape genetics is an increasingly powerful tool to identify marine environmental features that can be correlated with the genetic structure of species (Liggins et al. 2013). The influence of environmental variables on population subdivision can be measured using two different approaches: (1) comparing simulations of physical oceanographic models, e.g. based on ocean currents and larval characteristics, with genetic data represent a powerful tool for predicting larvae dispersal (e.g. Galindo et al. 2006, Galindo et al. 2010); and (2) assessing the influence of environmental factors such as sea surface temperature and tidal current on geographic genetic variation, i.e. testing the hypothesis that environmental features can explain the genetic structure of populations (GonzálezWangüemert et al. 2010; Wei et al. 2013b).

When inferring gene flow, it is also essential to consider different spatial scales. Important factors affecting dispersal might not be obvious in a large spatial scale study, since seascape effects on migration and genetic drift might be difficult to distinguish from microevolutionary forces such as mutations. On the other hand, studies considering only a 
fine spatial scale can be affected by noise in the genetic data associated with population subunits that persist across generations (intrademic structure). Therefore, the choice of sampling scale introduces different features that may affect interpretations of patterns of gene flow (Conover et al. 2006; Anderson et al. 2010).

Temporal variation in genetic structure can also be the result of environmental factors and it is very well documented in many marine invertebrates (e.g. Kovach et al. 2010; Perrier et al. 2013; Owen \& Rawson 2013; Kesäniemi et al. 2014; Pusack et al. 2014). Environmental conditions may lead to 'sweepstake recruitment', where random events determine which adults are successful each spawning season (Hedgecock 1994). Variation in oceanic currents can also affect the movement of larvae and consequently the patterns of genetic structure of species (Sotka et al. 2004; Selkoe \& Toonen 2006). These complex recruitment dynamics, which are characteristic of many marine invertebrate species, have very important consequences for fisheries management. However this represents a challenge for assessing the size of the fishable component of stocks, which is dependent on the successful recruitment of individuals. Therefore, population genetic studies at different spatial and temporal scales can potentially provide a clearer interpretation of the factors influencing population dynamics.

Finally, historical environmental processes such as glacial periods and changes in sea level can also affect the distribution of species and consequently lead to the observed population genetic structure (Gaggiotti et al. 2009). For example, in New Zealand several species have shown a genetic break at around $41-42^{\circ} \mathrm{S}$, just south of the Cook Strait (Gardner et al. 2010) and studies suggest that this genetic split is the result of historic land subdivision combined with the complex hydrography of the Cook Strait region (Apte \& Gardner 2002; Goldstien et al. 2006). Therefore, historical barriers can restrict connectivity between populations even if currently connected by strong oceanic currents, such as observed for a reef system in Indonesia where the patterns of genetic structure reflect the separation of ocean basins during the Pleistocene low-sea-levels (Barber et al. 2000).

\section{The New Zealand marine environment}

New Zealand is an archipelago of more than 700 islands with a complex oceanography. Extending from the subtropical Kermadec Islands at $29^{\circ} \mathrm{S}$ to the subantarctic 
Campbell Islands at $52^{\circ} \mathrm{S}$, it is located in the subtropical convergence, the division between subtropical water in the north and subantarctic water in the south (Wallis \& Trewick 2009). The complexity of the region, for example due to several small currents and eddies, variable wind, waves and tides as well as coastal currents shaped by interactions with ocean floor topography, makes it difficult to predict the influence of the marine environment on marine populations structure (Ross et al. 2009). On the other hand, it is an ideal system to investigate the influence of these current systems and environmental variability on patterns of genetic structure and connectivity in coastal marine species.

Identifying spatial and temporal genetic boundaries across species in the New Zealand marine environment can bring insights about the processes that shape patterns of genetic structure and marine connectivity at the ecosystem level, which is required for management efforts. Fifty-eight studies concerning the genetic structure of 42 coastal marine taxa were conducted during the past 32 years in New Zealand (Gardner et al. 2010). Twenty (33\%) of these studies reported north-south divergence, 16 (28\%) reported no genetic structure among populations, $12(21 \%)$ reported divergence within and/or among populations, 9 $(16 \%)$ reported isolation by distance and one (2\%) reported east-west divergence. Studies that described north-south differentiation frequently reported the location of the genetic break at around $41-42^{\circ} \mathrm{S}$, just south of the Cook Strait (Gardner et al. 2010).

Gardner et al. (2010) drew attention to gaps in knowledge concerning coastal population structure particularly in estuarine and soft substrate habitats. To fully understand the patterns of regional connectivity in New Zealand and characterize the geographic units of genetic diversity for conservation, these gaps in knowledge must be addressed. Furthermore, knowledge of population connectivity among these fragile habitats is essential because they hold assemblages of commercially important species and are subjected to intense human activity, such as dredging and port activities (Cole et al. 2000).

\section{Study species}

The New Zealand scallop, Pecten novaezelandiae, is a large bivalve with a flat upper left valve and a convex lower right valve. Growth to $100 \mathrm{~mm}$ shell length takes between 1.5 and 3.5 years although growth rates are spatially and temporally variable. Morrison (1999) 
observed that the maximum average size and average growth rates diminish with increasing depth and the maximum reported age in unexploited populations is 7 years.

The New Zealand scallop is an exceptionally fecund hermaphroditic species. Size at full maturity is reported at $65 \mathrm{~mm}$ shell height in the Hauraki Gulf and the smallest mature individuals reported are $55 \mathrm{~mm}$ shell height (Williams \& Babcock 2005). However, studies suggest that scallops might reach maturity at different sizes in different locations (Williams \& Babcock 2005). This species generally breeds in early summer even though partial spawning may occur from August to February (Williams \& Parkinson 2010) and individual populations are usually synchronous, i.e. individuals spawn at the same time (Shumway \& Parsons 2006). Factors such as latitude, depth and temperature may influence the spawning dynamics of $P$. novaezelandiae. For example, it has been demonstrated for other scallop species, such as Argopecten irradians and Pecten maximus, that higher latitude populations spawn earlier in the season and over longer periods of time (reviewed in Shumway \& Parsons 2006). Tunbridge (1968) showed that Pecten novaezelandiae spawned first in the deeper beds (28-40 m) among seven sites ranging from 6-40 m depth. Finally, evidence suggests that elevated food (chlorophyll $a$ ) levels and temperature can also influence spawning dynamics in Argopecten purpuratus, populations of which exhibited intense spawning during an El Niño period (Shumway \& Parsons 2006).

The endemic New Zealand scallop is an epibenthic organism found in diverse types of soft substrate from low tide to $90 \mathrm{~m}$ deep and patchily distributed throughout coastal waters (Bull 1976; Williams \& Parkinson 2010). Similarly to other scallops, P. novaezelandiae is able to move quickly by clapping its valves, thereby propelling the animal forward. However, most species become rapidly exhausted after moving short distances (Morrison 1999) which possibly means that they are potentially unable to achieve movement on the large scale of large beds and therefore the main dispersal period occurs through the larval stage. The duration of scallop larvae stages generally ranges from 6 - 70 days (Shumway \& Parsons 2006) and has been shown to vary within species depending on temperature (Beaumont \& Barnes 1992). The estimated pelagic larval duration of $P$. novaezelandiae is three to four weeks (Bull 1976; Jong 2013), which is within the range described for other species such as 25 days for Pecten maximus (Pennec et al. 2003; Figure 1.1). 


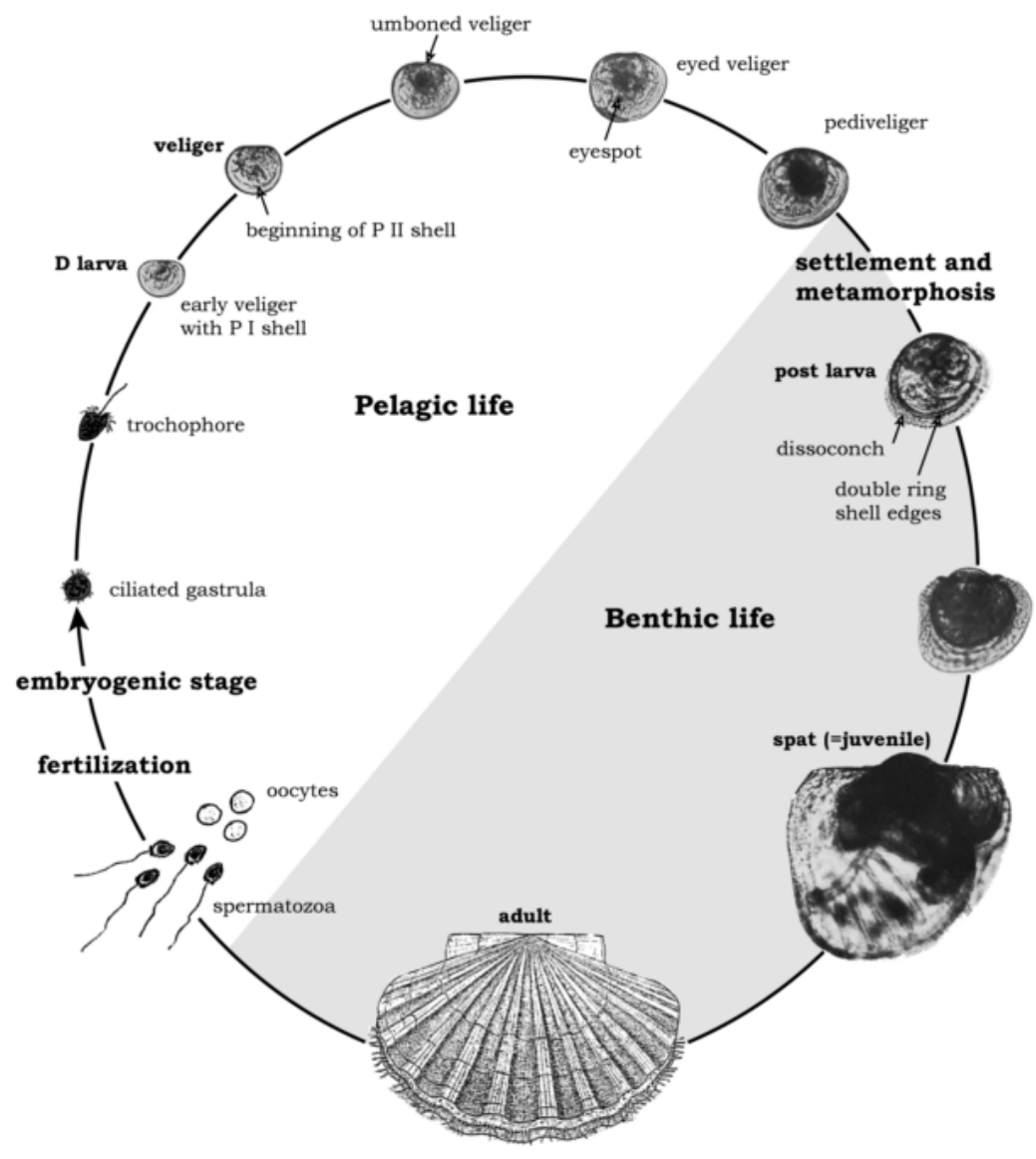

Figure 1.1 Diagrammatic representation of the life cycle of Pecten maximus (Pennec et al. 2003)

The New Zealand scallop is a highly valued species, supporting important commercial, recreational and customary fisheries. However, its exploitation faces several problems mainly because its recruitment is highly variable and the processes that result in large periodic population fluctuations are poorly understood (Ministry of Primary Industries 2013a). Spat variability can also be the result of human-induced processes such as fishingrelated changes to habitat that affect juvenile scallop survival and an increase in sedimentation input, which can affect adults and consequently reduce the likelihood of future larval settlement (Talman et al. 2004; Szostek et al. 2013). In New Zealand, evidence of declining stocks is found: (1) in the Coromandel fishery, which has been declining in terms of recruited biomass since 2005-06 (Williams \& Parkinson 2010); (2) in Golden Bay, which was effectively closed to commercial fishing in 2011 and in 2012 two scallop stocks or sub-stocks were considered to be overfished; and (3) in Tasman Bay, 
which has undergone a voluntary closure to commercial fishing since 2006 due to fishery collapse (Ministry of Primary Industries, 2012).

Currently, the New Zealand scallop is managed under the Quota Management System (QMS). The New Zealand Ministry of Primary Industries (MPI) works closely with stakeholders to establish a sustainable and cautious Total Allowable Commercial Catch (TACC) for each quota management area (QMA; Figure 1.2). Thus, an annual pre-season research survey is carried out to estimate abundance and determine sustainable yields and to determine the quantity that the commercial fishery can catch of each stock or QMA in that year. Additionally, there is also a size limit, measured in terms of shell length, which varies accordingly to each QMA (Williams \& Parkinson 2010). However, there is little information on the stock structure and population dynamics of this species and this substantial uncertainty may lead to inappropriate estimates of TACC.

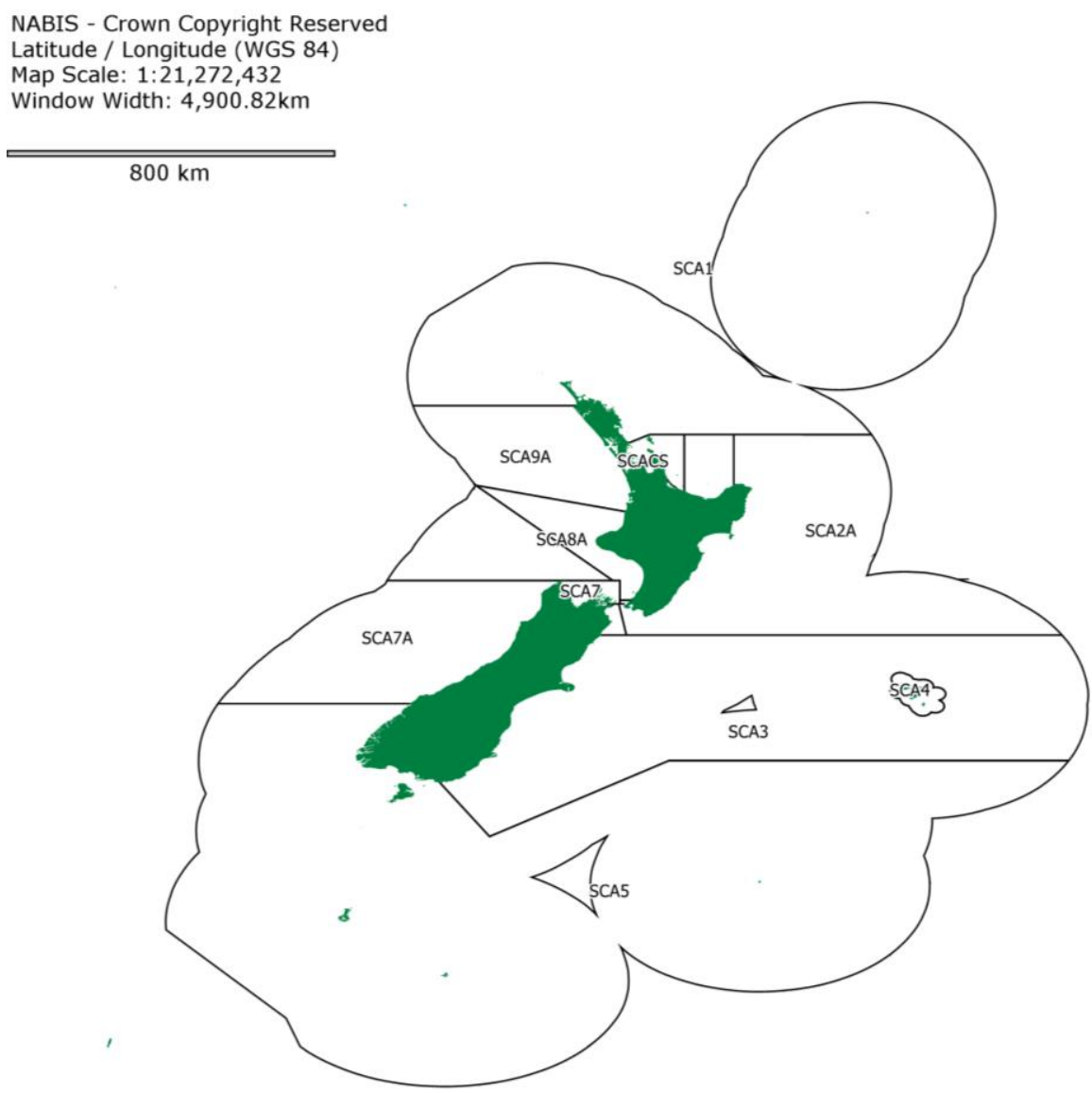

Figure 1.2 Map of the location of quota management areas (QMAs) used for management of the commercial and recreational fishery of Pecten novaezelandiae (sourced from www.nabis.govt.nz) 


\section{Aims and thesis structure}

The overall aim of this $\mathrm{PhD}$ thesis was to investigate the patterns of connectivity and genetic structure of the endemic scallop Pecten novaezelandiae. This study brings together information for management of the New Zealand scallop and also knowledge about population dynamics of scallops in general, an important step to fully understand the processes that regulate scallops biomass fluctuations and to achieve sustainable fisheries management. It also provides information about the patterns of gene flow and the processes shaping those patterns in the New Zealand marine environment. Specific objectives and hypotheses tested included:

1) To develop and characterize a set of microsatellite markers for P. novaezelandiae;

2) To characterize the genetic variability of populations of $P$. novaezelandiae encompassing the species' range; evaluate the genetic differentiation and understand factors shaping the patterns found; test the null hypothesis of panmixia; and compare the observed patterns with other species, based on hypotheses of evolutionary age;

3) To identify environmental variables likely to explain the observed patterns of genetic structure across the species' range; test the null hypothesis that environmental variables are not correlated with genetic variation;

4) To estimate spatial and temporal genetic variation of $P$. novaezelandiae at a small scale (within the limits of the Coromandel fishery) using microsatellites and a hydrodynamic model; test the null hypothesis of panmixia and temporal stability; and study the possibility of a model of source-sink population dynamics.

This work is part of the project "Multi-species coastal marine connectivity" (ZBD2009_10) funded by the Ministry of Primary Industries (MPI), formerly Ministry of Fisheries. Sampling comprised small and large scale dredging and diving carried out from February 2012 until August 2014.

Each data chapter of this thesis (i.e. except Chapters 1 and 6) represents a different unit with specific objectives and individual sections of Introduction, Methods, Results and Discussion. The purpose of Chapter 1 (present chapter) is to give a general introduction to the research topics and an overview on the approaches used. Chapter 2 describes the 
microsatellites markers developed for $P$. novaezelandiae from 454 sequence data and corresponds to a manuscript published in Conservation Genetics Resources "Silva CNS, Gardner JPA (2014). Development and characterisation of 12 microsatellite markers for the New Zealand endemic scallop Pecten novaezelandiae. 6(2), 327-328". In Chapter 3 the genetic structure of $P$. novaezelandiae is described, the null hypothesis of panmixia (i.e., no genetic structure) is tested and the factors that shape the patterns observed are characterized. In Chapter 4 a seascape genetics approach is used to identify associations between the genetic structure of the New Zealand scallop across its distribution range and nine environmental variables. Chapter 5 describes a spatial and temporal analysis of dispersal of $P$. novaezelandiae in the Coromandel fishery region, using microsatellites integrated with hydrodynamic modelling to test the null hypothesis of panmixia. Finally, Chapter 6 summarizes the key findings, indicates future directions and provides recommendation about the sustainable management of the New Zealand scallop. 


\section{CHAPTER 2}

\section{Development of microsatellite DNA markers for the New}

\section{Zealand scallop Pecten novaezelandiae}

Manuscript published in Conservation Genetics Resources:

Silva CNS, Gardner JPA (2014). Development and characterisation of 12 microsatellite markers for the New Zealand endemic scallop Pecten novaezelandiae.

Conservation Genetics Resources 6(2), 327-328. 


\section{Introduction}

The New Zealand scallop (Pecten novaezelandiae Reeve, 1853), (Bivalvia: Pectinidae) is distributed throughout coastal and semi-estuarine waters in the North, South, Stewart and Chatham Islands. It is a highly valued species supporting important fisheries although stocks have been declining and there is still substantial uncertainty about stock status (Williams \& Parkinson 2010). Despite its ecological and economic importance, there is still a lack of genomic resources for the New Zealand scallop and no studies of population connectivity have been conducted to date.

Marine species commonly have vast population sizes and widespread dispersal capabilities and the expectations are populations exhibiting high levels of genetic diversity. Therefore, studies of population connectivity and stock structure require sensitive tools to detect low levels of differentiation such as the use of microsatellite markers (Avise 2004). Microsatellites are currently the most popular and versatile DNA markers for ecological applications. With the advances in next generation sequencing (NGS) technologies, the development of microsatellite markers has become cheaper, easier and much faster compared to traditional methods (Abdelkrim et al. 2009). A commonly used NGS technology uses the GS-FLX Titanium platform (Roche 454 Life Science, USA) and is generally named as 454 sequencing.

The analysis of PCR products by laser detection requires one of the primers to be fluorescently labelled and these dyes are very expensive. To overcome this issue, Schuelke (2000) developed the M13 protocol which enables a single set of fluorescently labelled markers to be used in conjunction with a range of PCR primers and it can also be multiplexed (PCR-amplified as a multiplex of loci) or pooled together after single PCRs (pool-plexed).

In this study, microsatellite markers were developed de novo for the New Zealand scallop Pecten novaezelandiae using NGS (454 sequencing). These markers were developed with the intention of investigating genetic diversity and population structure connectivity among populations. 


\section{Materials and methods}

High quality genomic DNA was extracted from the adductor muscle of one individual of Pecten novaezelandiae collected from Wellington Harbour using a Phenol-ChloroformIsoamyl method. In order to precipitate the polysaccharides present in the mucus, a volume of $100 \mu \mathrm{L}$ of saturated $\mathrm{KCl}$ solution $(40 \mathrm{~g} / 100 \mathrm{ml})$ was added to the digested DNA. Samples were sent to the High-Throughput DNA Sequencing Unit at University of Otago (Dunedin, New Zealand) for microsatellite-enriched library preparation and 454 sequencing on a Roche GS-FLX instrument. The software iQDD V1.3 (Meglécz et al. 2010) was used to search for Short Tandem Repeats (STRs) and design possible primers for PCR amplification, setting $90 \mathrm{bp}$ as minimum PCR product size and $100 \mathrm{bp}$ as minimum sequence length. The minimum repeat length for di-, tri-, tetra- and pentanucleotide motifs was set to $8,6,4$ and 4 , respectively. The best primer pair for each repeat sequence was selected and a total of 49 primers were retained for PCR amplification.

A M13 (5'-TGT AAA ACG ACG GCC AGT) tail was added to the 5' end of each forward primer for detection purposes following Schuelke (2000). Four primers each labelled with a fluorescent dye with the same M13 primer sequence were ordered. The fluorochrome labels 6-FAM, NED, PET and VIC were used: 5'-FAM- ACG ACG TTG TAA AAC GAC-3', 5'-NED- ACG ACG TTG TAA AAC GAC-3', 5'-PET- ACG ACG TTG TAA AAC GAC-3', 5'-VIC- ACG ACG TTG TAA AAC GAC-3'. PCR amplification conditions were: $94^{\circ} \mathrm{C}(5 \mathrm{~min}), 30$ cycles at $94^{\circ} \mathrm{C}(30 \mathrm{~s}) / 56^{\circ} \mathrm{C}(45 \mathrm{~s}) / 72^{\circ} \mathrm{C}$ (45 s), followed by 8 cycles $94^{\circ} \mathrm{C}(30 \mathrm{~s}) / 53^{\circ} \mathrm{C}(45 \mathrm{~s}) / 72^{\circ} \mathrm{C}(45 \mathrm{~s})$, and a final extension at $72^{\circ} \mathrm{C}$ for $10 \mathrm{~min}$ (Schuelke 2000). Each reaction $(15 \mu \mathrm{L})$ contained $70 \mathrm{ng}$ of DNA template, 0.5 units/ $\mu \mathrm{L}$ Taq DNA polymerase, $67 \mathrm{mM}$ Tris-Hcl pH 8.8, $16 \mathrm{mM}\left(\mathrm{NH}_{4}\right) \mathrm{SO}_{4}, 2 \mathrm{mM}$ $\mathrm{MgCl}_{2}, 0.2 \mathrm{mM}$ dNTPs, $0.075 \mu \mathrm{M}$ of Forward primer, $0.3 \mu \mathrm{M}$ of Reverse and M13 primers and $\mathrm{ddH}_{2} \mathrm{O}$ to volume.

After locus validation, 12 loci were selected and successfully combined in four multiplex groups (Table 2.1) using the software Multiplex Manager 1.0 (Holleley \& Geerts 2009). Conditions of the PCR amplification were as follows: $94^{\circ} \mathrm{C}(5 \mathrm{~min})$, then 36 cycles at $94^{\circ} \mathrm{C}(30 \mathrm{~s}) / 60^{\circ} \mathrm{C}(45 \mathrm{~s}) / 74^{\circ} \mathrm{C}(60 \mathrm{~s})$ and a final extension at $74^{\circ} \mathrm{C}(10 \mathrm{~min})$. Each reaction $(15 \mu \mathrm{L})$ contained $70 \mathrm{ng}$ of DNA template, 0.5 units/ $\mu \mathrm{L}$ Taq DNA polymerase, 67 $\mathrm{mM}$ Tris- $\mathrm{HCl} \mathrm{pH} 8.8,16 \mathrm{mM}\left(\mathrm{NH}_{4}\right) \mathrm{SO}_{4}, 2 \mathrm{mM} \mathrm{MgCl}_{2}, 0.2 \mathrm{mM}$ dNTPs, $0.3 \mu \mathrm{M}$ of Forward and Reverse primers and $\mathrm{ddH}_{2} \mathrm{O}$ to volume. A total of 32 STRs (65\%) were 
successfully amplified. PCR products were sent for genotyping on an ABI3730 Genetic Analyzer (Macrogen Inc., Korea) and fragment sizes were scored manually. To improve cost-effectiveness, the PCR products were mixed together in two groups and analyzed simultaneously (PCR pool-plexing). The markers were tested for polymorphism in 122 randomly selected individuals collected from Little Barrier Island $\left(-36^{\circ} 13^{\prime} 51.0^{\prime \prime},+175^{\circ} 4^{\prime}\right.$ $\left.17.40^{\prime \prime}\right)$ and Wellington Harbour ( $\left.-41^{\circ} 18^{\prime} 05.0^{\prime \prime},+174^{\circ} 48^{\prime} 35.0^{\prime \prime}\right)$ and basic statistics were calculated for each locus. Microchecker (Van Oosterhout et al. 2004) was used to check for null alleles, stuttering and large allele dropout. Tests of linkage disequilibrium, departure from Hardy-Weinberg equilibrium (HWE), estimates of diversity and the expected and observed heterozygosities $\left(\mathrm{H}_{\mathrm{e}}\right.$ and $\mathrm{H}_{\mathrm{o}}$ ) were performed in GENEPOP (Rousset 2008).

\section{Results}

The runs yielded a total of 315,404 DNA sequences (Figure 2.1) and iQDD identified 448 perfect microsatellite motif sequences with primers. The best primer pairs were selected and a total of 49 primers were tested for PCR amplification. Loci were discarded if they failed to amplify, showed high levels of allelic drop out, were confounded by artefacts or were monomorphic. Of these, 12 loci gave consistent results and were retained. All loci were polymorphic and the number of alleles ranged from 6 to 37 (Table 2.1). No evidence of stuttering or large allele dropout was detected and no significant linkage disequilibrium was detected between locus pairs (GENEPOP v.4.2 using the Markov chain method and Fisher's exact test). The observed $\left(\mathrm{H}_{\mathrm{o}}\right)$ and expected $\left(\mathrm{H}_{\mathrm{e}}\right)$ heterozygosities ranged from 0.209 to 0.926 and from 0.318 to 0.961 , respectively. Three loci (Pnova_01, Pnova_24 and Pnova_27) showed significant deviation from HWE and evidence of null alleles. 

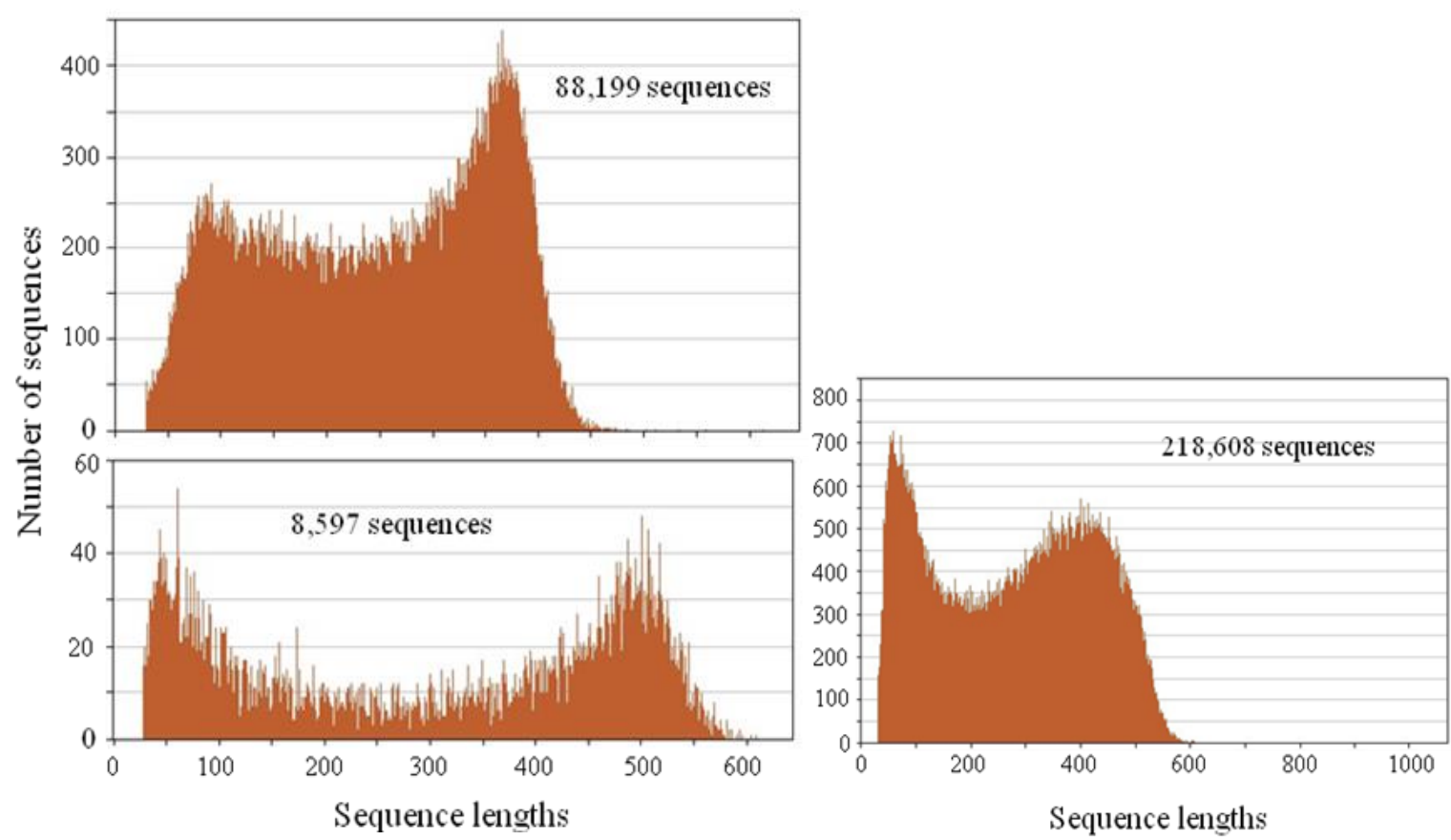

Figure 2.1 Length frequency of the 88199,8597 and 218608 reads, respectively, obtained with a 1/8th run on a 454 GS FLX instrument for Pecten novaezelandiae.

\section{Discussion}

The method used in this study allows a relatively rapid and affordable development of microsatellite markers. The next generation sequencing yields a vast amount of data (over 100,000 sequences produced by the 454 method) and the software iQDD V1.3 (Meglécz et al. 2010) scans potential primers, therefore prioritizing a potential number of primers before the laboratory testing phase. In addition, using the M13(-21) primer genotyping protocol (Schuelke 2000) and PCR pool-plexing can save thousands of dollars offering a better alternative for small research budgets.

Microsatellite development can be challenging particularly for highly fecund marine bivalves with high mutation rates. Previous studies have reported null alleles (i.e. alleles that fail to amplify by the polymerase chain reaction) in bivalves (Hedgecock et al. 2004; Marín et al. 2012) causing the underestimation of heterozygotes and consequently deviations from Hardy-Weinberg equilibrium (HWE) expectations. For that reason the 
markers Pnova_01, Pnova_24 and Pnova_27 should be used with caution having in mind the scale to which they might influence results.

The scallop Pecten novaezelandiae has been widely studied in New Zealand as an important and valued fishery resource (e.g. Williams and Babcock 2004; Williams and Babcock 2005). A few phylogenetic studies have been done to date to elucidate the evolutionary relationships and taxonomy of the family Pectinidae (Saavedra \& Peña 2006; Puslednik \& Serb 2008; Salvi et al. 2010). However, there was no previous information on the genetic structure of $P$. novaezelandiae. These newly developed microsatellite markers are particularly suitable for assessing current levels of genetic variability within and between populations of $P$. novaezelandiae as well as identifying the degree of differentiation between stocks and therefore are of great utility as a tool for informing conservation management. 
Table 2.1 Polymorphic microsatellite markers for $P$. novaezelandiae with locus characteristics, number of alleles $\left(\mathrm{N}_{\mathrm{a}}\right)$, observed heterozygosity $\left(\mathrm{H}_{\mathrm{o}}\right)$, expected heterozygosity $\left(\mathrm{H}_{\mathrm{e}}\right)$, probability of being out of Hardy-Weinberg Equilibrium (HWE).

\begin{tabular}{|c|c|c|c|c|c|c|c|c|c|}
\hline $\begin{array}{l}\text { Multiplex } \\
\text { group }\end{array}$ & Locus & $\begin{array}{c}\text { Accession } \\
\text { no. }\end{array}$ & Repeat & Forward primer sequence $\left(5^{\prime}-3^{\prime}\right)$ & $\begin{array}{l}\text { Allele size } \\
\text { range (bp) }\end{array}$ & $\mathrm{N}_{\mathrm{a}}$ & $\mathrm{H}_{\mathrm{o}}$ & $\mathrm{H}_{\mathrm{e}}$ & $\begin{array}{l}\text { HWE } \\
\text { p-value }\end{array}$ \\
\hline \multirow[t]{2}{*}{1 (Pool 1) } & Pnova_09 & KF732829 & $(\mathrm{AGTT})_{5}$ & GGCGTCCACTGACAGATAAG & $112-144$ & 7 & 0.351 & 0.349 & 0.870 \\
\hline & Pnova_24 & KF732831 & $(\mathrm{AGAC})_{5}$ & CACTGACGAAACGTTGGTGT & $116-140$ & 7 & 0.307 & 0.395 & 0.003 \\
\hline \multirow[t]{2}{*}{2 (Pool 1) } & Pnova_02 & KF732826 & $(\mathrm{TA})_{11}$ & CAGTAGCTCCTGCCCCATTA & $163-185$ & 11 & 0.661 & 0.695 & 0.792 \\
\hline & Pnova_27 & KF732832 & $(\mathrm{TATC})_{33}$ & CAGATACGTCAGAGTGCTGATTC & $191-343$ & 37 & 0.877 & 0.961 & 0.007 \\
\hline \multirow[t]{3}{*}{3 (Pool 1) } & Pnova_01 & KF732825 & $(\mathrm{TA})_{8}$ & GTAGCACACGCAAATGCCTA & $207-229$ & 6 & 0.209 & 0.377 & 0.000 \\
\hline & Pnova_04 & KF732827 & $(\mathrm{TTGT})_{7}$ & AATGCAGGAAGGTTTTGGTTT & $276-300$ & 7 & 0.681 & 0.627 & 0.312 \\
\hline & Pnova_28 & KF732833 & $(\mathrm{CAA})_{19}$ & AAGGCATATAACTCAGCTGGAA & $254-377$ & 34 & 0.926 & 0.959 & 0.138 \\
\hline \multirow{2}{*}{4 (Pool 2) } & Pnova_32 & KF732835 & $(\mathrm{AT})_{11}$ & GTGTCGTCTACAGCCGGAAT & $175-185$ & 6 & 0.458 & 0.496 & 0.626 \\
\hline & Pnova_33 & KF732836 & $(\text { AATAT })_{10}$ & CTCCAGGAGTAGAGCCGATG & $164-214$ & 10 & 0.691 & 0.789 & 0.380 \\
\hline
\end{tabular}


CHAPTER 3

Genetic structure of Pecten novaezelandiae throughout its distribution range 


\section{Introduction}

Many marine species have low levels of population genetic differentiation as a result of large population sizes, high fecundity and a pelagic larval stage (Cowen \& Sponaugle 2009). Pelagic larvae provide species with a high dispersal capability and larval transport is expected to play an important role in determining population structure (Hedgecock 1986). The maximum dispersal ability can be related to the pelagic larval duration and the distance able to be travelled during that time (Bohonak 1999). There are now many examples of species showing extensive gene flow and panmixia (Apte \& Gardner 2001; Neethling et al. 2008; Reisser et al. 2014), but it has become clear that even marine invertebrates with a long pelagic larval duration can exhibit significant genetic differentiation (Thomas \& Bell 2013). The trade-off between potential and realised gene flow has only recently begun to be appreciated (Shanks 2009; Dawson et al. 2014; Reisser et al. 2014).

There are many factors that influence the population genetic structure of species, particularly life history strategy and ocean currents (Shanks 2009), which highlights the importance of considering each species independently. Usually, the observed population genetic structure of a species is shaped by historical factors, such as past glaciations or changes in sea level, which leave a present genetic signature (Hewitt 1996), and contemporary factors such as current barriers to gene flow. Therefore, the degree of genetic variation between populations is the result of the interaction between gene flow, genetic drift, mutation, natural selection and historical processes (Gaggiotti et al. 2009).

For widely distributed species, populations at the edges of their distributions are expected to have lower abundance as they become more spatially isolated from the geographical centre of their distribution (Vucetich \& Waite 2003). This is commonly referred to as the 'abundant centre' model, which has been frequently discussed within the context of the ecology and evolution of species' ranges (Sagarin \& Gaines 2002). A resulting debate has ensued in evolutionary biology as to the degree to which the 'abundant centre' distribution model is expressed in the amount of genetic diversity among populations in the geographic range of the species. While some studies show a decline in genetic diversity and increased differentiation towards the species range limits (reviewed in Eckert et al. 2008), the mechanisms that create these patterns are not clear. Therefore, studies that can encompass the entire distribution range of a species can provide valuable 
information about the relative roles of historical and contemporary factors in shaping genetic variability and patterns of genetic structure (Hasselman et al. 2013).

New Zealand has a wide diversity of habitats and high levels of endemism. Its marine environment ranges from $34^{\circ}$ to $47^{\circ}$ latitude south, with subtropical to sub-polar characteristics and a very complex oceanography (see Gordon et al. 2010). In New Zealand most studies on the population genetic structure of marine organisms are of rocky reef species; studies of species living on sandy and soft bottom habitats are scarce (see Gardner et al. 2010). The endemic New Zealand scallop Pecten novaezelandiae supports a number of important fisheries but there is no previous information regarding its genetic structure. On the one hand, the species has traits commonly associated with high potential for dispersal, as a broadcast spawner with high fecundity (Williams \& Babcock 2004; Shumway \& Parsons 2006). In contrast, these scallops have characteristics that might be expected to lead to population differentiation since they are relatively long-lived ( $>7$ years), adults are sedentary and patchily distributed (Morrison 1999), and they have an estimated pelagic larval duration of approximately two to three weeks (Williams \& Babcock 2004; Shumway \& Parsons 2006). As a comparison, the New Zealand bivalve Paphies subtriangulata, which has a similar pelagic larval duration, showed evidence of strong differentiation between the Chatham Islands and the mainland populations (Hannan 2014), while Perna canaliculus with a longer pelagic larval duration (three to five weeks), shows north-south differentiation in New Zealand, with a genetic break at about $42^{\circ} \mathrm{S}$ latitude (Apte \& Gardner 2002).

A variety of patterns of population genetic structure have been observed in New Zealand. Gardner et al. (2010) categorised these patterns as: (1) no structure; (2) isolation by distance; (3) divergence within and among populations; (4) east-west divergence; and (5) north-south divergence. Most of the studies (33\%) reported a north-south split around the southern region of the Cook Strait, at about $42^{\circ}$ S. For some species, such as Perna canaliculus and Patiriella regularis, the reported divergence time between North and South Island populations dates to approximately 1.3 Ma ago (Apte \& Gardner 2002; Ayers \& Waters 2005), which corresponds to a period of hydrographic change across the Cook Strait region (Lewis et al. 1994). In contrast, the contemporary North and South Island lineages of each of the three endemic intertidal limpets Cellana ornata, Cellana radians and Cellana flava diverged approximately 200000 to 300000 years ago (Goldstien et al. 2006). 
While both historical and current factors are expected to shape existing patterns of genetic differentiation, examining their relative importance can be challenging. The recent evolutionary history of Pecten novaezelandiae might be indicative of a potentially weak genetic differentiation across its distributional range. Fossil records in New Zealand document the origin of the genus Pecten as a result of succession of populations during the Pleistocene, approximately $1 \mathrm{Ma}$ ago (Beu 2010). Therefore, the recent evolutionary history of the New Zealand scallop allows for testing of the effects of more recent evolutionary processes in shaping species population genetic structure. In addition, evaluating latitudinal changes in genetic diversity of Pecten novaezelandiae can provide more information on its colonization history and on recent factors affecting gene flow. Finally, as microsatellite markers are highly variable, they are especially useful for the interpretation of recent demographic events. In this study, the null hypothesis of panmixia of $P$. novaezelandiae was tested using 12 microsatellites (Silva \& Gardner 2014) and a range of statistical analyses.

\section{Materials and methods}

\section{Sample collections}

In total, 952 individuals of Pecten novaezelandiae were sampled throughout its distribution range between 2012 and 2014. Sampling was dependent on the hotspots of distribution of the species. Samples were collected from 15 populations (Figure 3.1) using dredging from depths between $15-50 \mathrm{~m}$ and SCUBA diving from depths between $7-15$ m. The number of individuals per site varied between 10 and 99 (Table 3.1). After collection, scallops were measured (length of the top shell), size structure was analysed using the software R 3.1.0 (R Core Team 2014) and samples of the adductor muscle were preserved in $80 \%$ ethanol and frozen $\left(-20^{\circ} \mathrm{C}\right)$ for later processing. 


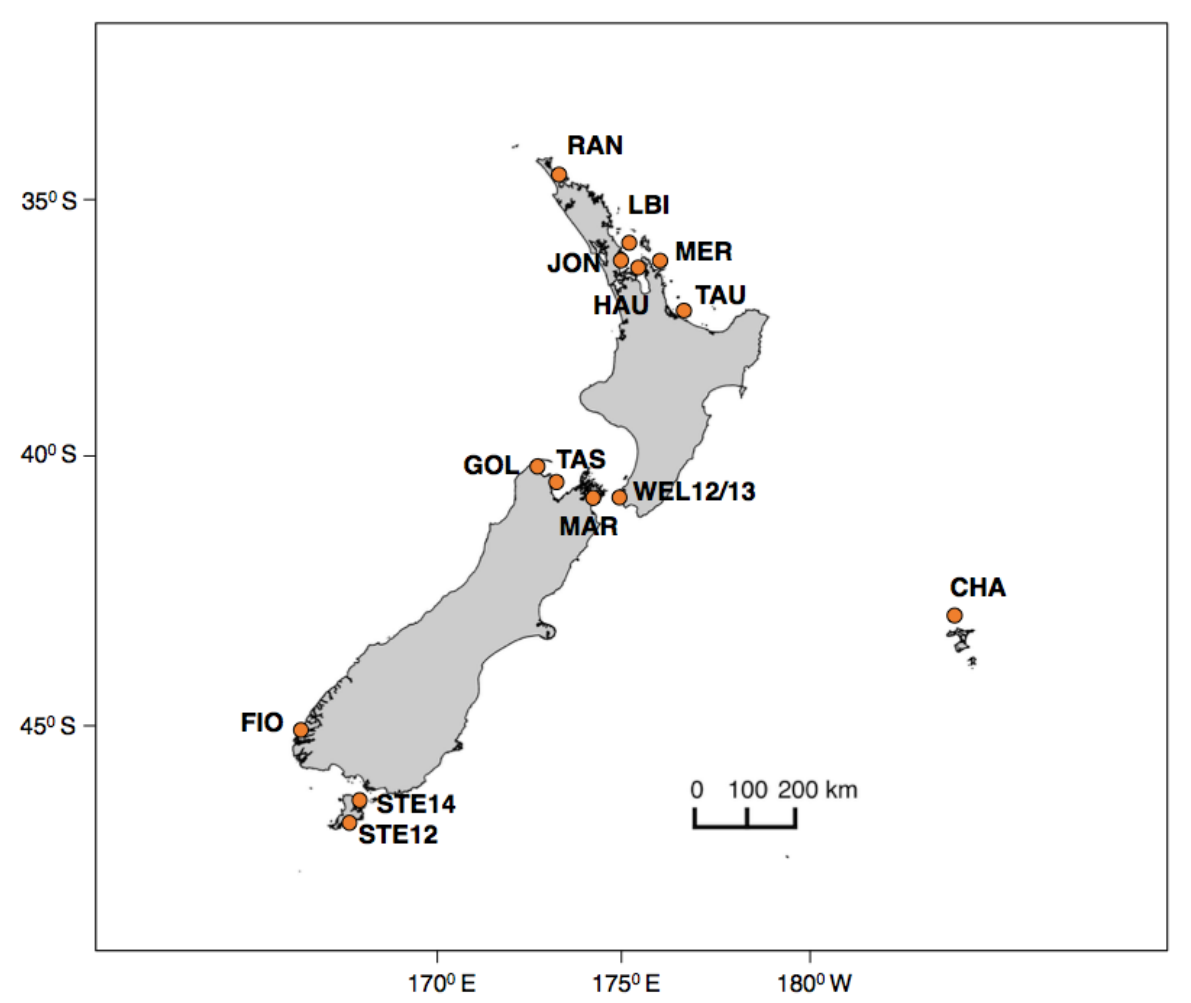

Figure 3.1 Map of sampling sites of Pecten novaezelandiae collected from New Zealand. Abbreviations as per Table 3.1

Table 3.1 Pecten novaezelandiae collecting site descriptions, sample sizes $(\mathrm{N})$, mean depth (m), dates and geospatial coordinates

\begin{tabular}{ccccccc}
\hline Code & Site Description & N & $\begin{array}{c}\text { Depth } \\
(\mathbf{m})\end{array}$ & $\begin{array}{c}\text { Date } \\
\text { collected }\end{array}$ & Latitude & Longitude \\
\hline RAN & Rangaunu Bay & 98 & 42 & $3 / 11 / 2012$ & $-34^{\circ} 49^{\prime} 2.8^{\prime \prime}$ & $+173^{\circ} 18^{\prime} 6.45^{\prime \prime}$ \\
LBI & Little Barrier Is & 95 & 15 & $1 / 05 / 2012$ & $-36^{\circ} 13^{\prime} 51.0^{\prime \prime}$ & $+175^{\circ} 44^{\prime} 17.40^{\prime \prime}$ \\
JON & Jones Bay & 40 & 10 & $15 / 11 / 2012$ & $-36^{\circ} 22^{\prime} 53.6^{\prime \prime}$ & $+174^{\circ} 48^{\prime} 59.48^{\prime \prime}$ \\
HAU & Hauraki Gulf & 99 & 43 & $28 / 11 / 2012$ & $-36^{\circ} 34^{\prime} 9.60^{\prime \prime}$ & $+175^{\circ} 14^{\prime} 6.57^{\prime \prime}$ \\
MER & Mercury Is & 69 & 15 & $1 / 05 / 2012$ & $-36^{\circ} 41^{\prime} 3.60^{\prime \prime}$ & $+175^{\circ} 43^{\prime} 33.60^{\prime \prime}$ \\
TAU & Tauranga & 99 & 22 & $3 / 05 / 2012$ & $-37^{\circ} 40^{\prime} 14.4^{\prime \prime}$ & $+176^{\circ} 24^{\prime} 10.80^{\prime \prime}$ \\
WEL12 & Wellington Harbour & 67 & 10 & $3 / 02 / 2012$ & $-41^{\circ} 18^{\prime} 05.0^{\prime \prime}$ & $+174^{\circ} 48^{\prime} 35.0^{\prime \prime}$ \\
WEL13 & Wellington Harbour & 48 & 10 & $31 / 05 / 2013$ & $-41^{\circ} 18^{\prime} 05.0^{\prime \prime}$ & $+174^{\circ} 48^{\prime} 35.0^{\prime \prime}$ \\
MAR & Marlborough Sounds & 50 & 15 & $11 / 12 / 2012$ & $-41^{\circ} 11^{\prime} 54.8^{\prime \prime}$ & $+174^{\circ} 07^{\prime} 09.2^{\prime \prime}$ \\
TAS & Tasman Bay & 48 & 22 & $13 / 05 / 2014$ & $-41^{\circ} 04^{\prime} 27.0^{\prime \prime}$ & $+173^{\circ} 05^{\prime} 48.0^{\prime \prime}$ \\
GOL & Golden Bay & 48 & 20 & $14 / 05 / 2014$ & $-40^{\circ} 36^{\prime} 27.6^{\prime \prime}$ & $+172^{\circ} 46^{\prime} 40.8^{\prime \prime}$ \\
FIO & Fiordland & 35 & 9 & $1 / 10 / 2012$ & $-45^{\circ} 35^{\prime} 7.51^{\prime \prime}$ & $+166^{\circ} 44^{\prime} 4.34^{\prime \prime}$ \\
STE12 & Stewart Is, Port Pegasus & 10 & 10 & $11 / 05 / 2012$ & $-47^{\circ} 10^{\prime} 6.53^{\prime \prime}$ & $+167^{\circ} 42^{\prime} 0.97^{\prime \prime}$ \\
STE14 & Stewart Is, Paterson Inlet & 48 & 7 & $4 / 04 / 2014$ & $-46^{\circ} 55^{\prime} 58.0^{\prime \prime}$ & $+168^{\circ} 04^{\prime} 15.0^{\prime \prime}$ \\
CHA & Chatham Is & 98 & 50 & $11 / 03 / 2013$ & $-43^{\circ} 42^{\prime} 32.40^{\prime \prime}$ & $-176^{\circ} 23^{\prime} 38.40^{\prime \prime}$ \\
\hline Total & & 952 & & & & \\
\hline
\end{tabular}




\section{Laboratory protocols}

Total DNA was extracted from the adductor muscle using Geneaid Genomic DNA Mini Kits (Tissue) following the manufacturer's instructions. DNA concentrations and the A260/A280 ratios were quantified using a NanoDrop ${ }^{\text {TM }}$ ND-1000 (Thermo Scientific). Specimens were genotyped at 12 polymorphic microsatellite loci: Pnova_01, Pnova_02, Pnova_04, Pnova_05, Pnova_09, Pnova_10,Pnova_24, Pnova_27, Pnova_28, Pnova_31, Pnova_32, Pnova_33 (Silva \& Gardner 2014). Microsatellite loci were PCR-amplified in reactions $(15 \mu \mathrm{L})$ containing $70 \mathrm{ng}$ of DNA template, 0.5 units $/ \mu \mathrm{L}$ Taq DNA polymerase, $67 \mathrm{mM}$ Tris- $\mathrm{HCl} \mathrm{pH} 8.8,16 \mathrm{mM}\left(\mathrm{NH}_{4}\right) \mathrm{SO}_{4}, 2 \mathrm{mM} \mathrm{MgCl}_{2}, 0.2 \mathrm{mM} \mathrm{dNTPs}, 0.3 \mu \mathrm{M}$ of Forward and Reverse primers and $\mathrm{ddH}_{2} \mathrm{O}$ to volume. Based on the combinations recommended by the Multiplex Manager 1.0 program (Holleley \& Geerts 2009), primer pairs were PCR multiplexed using the conditions described in Chapter 2, Supplementary Table 1. Primers were fluorescently labelled (FAM, NED, PET and VIC) and groups 1, 2 and 3 were pool-plexed. PCR products were visualized with an automated sequencer (ABI PRISM 3730 DNA Sequencer, Applied Biosystems) with the GeneScan-500 (LIZ) internal size standard. The software GeneMarker V2.2.0 (SoftGenetics) was used to analyse electropherograms for allele scoring and the alleles were binned with manual checking using the AutoBin program (Guichoux et al. 2011).

\section{Analysis of genetic diversity}

Genotyping artefacts were assessed using the software Micro-Checker v.2.2.0.3 (Van Oosterhout et al. 2004). Analysis of departure from Hardy-Weinberg equilibrium (HWE) and linkage disequilibrium (LD) were performed using the software GenePop on the web using the Markov chain method and Fisher's exact test (Rousset 2008). False discovery rate (FDR) control (Verhoeven et al. 2005) was applied to p-values in all statistical analyses that included multiple comparisons.

To determine whether sufficient sample size was achieved to accurately represent allelic variation, allele discovery curves were computed for the twelve loci using the package "PopGenKit" (Rioux Paquette 2011) in R 3.1.0 (R Core Team 2014). Data were combined from all populations for this analysis. 
An outlier analysis was performed using the software Lositan (Antao et al. 2008), which identifies any outlier loci that have excessively high or low $F_{\mathrm{ST}}$ values compared to neutral expectations, considering the relationship between $F_{\mathrm{ST}}$ and $\mathrm{H}_{\mathrm{E}}$ (expected heterozygosity) in an island model. Fifty thousand simulations were run with a confidence interval (CI) of 0.95 under the infinite alleles model (IAM) for a sample size of 50 . The average neutral $F_{\mathrm{ST}}$ was used and the "force mean $F_{\mathrm{ST}}$ " option was selected to increase the reliability of mean $F_{\text {ST. }}$

The software HP-Rare (Kalinowski 2005) was used to quantify genetic diversity as allelic richness $\left(A_{R}\right)$ and private allelic richness $\left(P A_{R}\right)$ with a rarefaction sample size of 24 individuals and a minimum sample size of 16 genes due to missing data. GenAlEx 6.5 (Peakall \& Smouse 2012) was used to quantify the number of private alleles per site (Pa), observed $\left(\mathrm{H}_{\mathrm{O}}\right)$ and expected $\left(\mathrm{H}_{\mathrm{E}}\right)$ heterozygosity, and the fixation index $\left(\mathrm{F}_{\mathrm{IS}}\right)$.

\section{Population structure}

Genetic differentiation among populations was assessed using different approaches. Pairwise $F_{\mathrm{ST}}$ values were calculated using the software GenePop on the web (Rousset $2008)$. This $F_{\mathrm{ST}}$ value, formally known as theta $(\theta)$, was adapted by Weir \& Cockerham (1984) for using with multiallelic loci, it is unbiased to different sample sizes and uses an ANOVA approach to standardize variation within and among populations. An Exact G-test (Goudet et al. 1996) was also calculated in GenePop (Markov-chain parameters: 10,000 dememorization steps, 1,000 batches and 10,000 iterations per batch) for each population pair using the $\mathrm{G}$ log likelihood ratio. All p-values were corrected using the False Discovery Rate (FDR) (Verhoeven et al. 2005) and comparisons were considered significant at $\mathrm{p}<$ 0.05 .

Pairwise $\mathrm{R}_{\mathrm{ST}}$ values were calculated using the software Arlequin 3.5.1.3 (Excoffier \& Lischer 2010) with 10,000 permutations for significance. $\mathrm{R}_{\mathrm{ST}}$ is an $F_{\mathrm{ST}}$ analogue (Slatkin 1995) that considers a stepwise mutation model in microsatellite loci, meaning that each mutation changes an allele's length by only one repeat unit. All p-values were adjusted for multiple comparisons using FDR (Verhoeven et al. 2005).

Modified pairwise statistics $\left(F^{\prime}\right.$ ST $)$ were calculated using the software GenoDive 2.0b25 (Meirmans \& Van Tienderen 2004). The $F_{\text {ST }}^{\prime}$ index is based on the AMOVA (analysis of 
molecular variance) framework (Excoffier et al., 1992). It is a standardised measure based on a maximum possible value given the observed amount of within-population diversity and therefore has no p-value associated with it.

Pairwise Jost's D values (Jost 2008) were calculated using the software GenoDive $2.0 \mathrm{~b} 25$ (Meirmans \& Van Tienderen 2004). Jost (2008) proposed the $\mathrm{D}_{\mathrm{ST}}$ metric, which takes total variation and differentiation into account and has no p-value associated with it.

Powsim 4.1 (Ryman \& Palm 2006) was used to assess the statistical power and estimate the probability of detecting differentiation at $F_{\mathrm{ST}}$ values of $0.001,0.0025,0.005$ and 0.01 . Using 20 replications, the $\chi^{2}$ and Fisher's exact tests were used for a set of simulations with different combinations of $N_{e f}$ (effective population size) and $t$ (generations of drift).

A principal components analysis (PCoA) was performed in GenAlEx 6.5 (Peakall \& Smouse 2006) to examine the variation among populations using co-dominant genetic distance. GenAlEx 6.5 (Peakall \& Smouse 2012) was also used to conduct the Analysis of Molecular Variation (AMOVA) using 999 permutations. The sampling sites were grouped according to the PCoA and the $F_{\mathrm{ST}}$ values. The 15 populations were grouped into 4 regions (North: RAN, LBI, JON, HAU, MER, TAU; Central: WEL12, WEL13, MAR, TAS, GOL; South: FIO, STE12, STE14; and Chatham Islands: CHA). An AMOVA was also conducted in GenAlEx 6.5 for the populations WEL12 and WEL13 to test if the temporal sampling differences (2012 versus 2013) explained any of the variation in the Wellington Harbour dataset. The following analyses were conducted using WEL as one single population because temporal differences did not explain any of the variation in the datasets of WEL12 and WEL13 (see Results section).

The neighbor joining (NJ) method (Saitou \& Nei 1987) was used to generate a population tree using the software PopTreeW (Takezaki et al. 2014) based on $F_{\text {ST }}$ values with 1000 bootstrap replicates.

The software package AWclust (Gao \& Starmer 2008) was used to evaluate population genetic structure among populations. AWclust is a nonparametric analysis and therefore does not assume HWE or linkage disequilibrium. This analysis was specifically designed for SNPs, so each microsatellite allele at each locus was coded as zero, one (heterozygous) or two (homozygous), following the approach of Gruber et al. (2013) and Wei et al. (2013a). The gap statistic was calculated for values of K ranging from 1 to 14 using 100 
null simulations. As a comparison, Bayesian cluster analysis was performed in Structure 2.3.4 (Pritchard et al. 2000) using 100,000 interactions, a burn-in length of 10,000, 'admixture' model, 'correlated allele frequencies' and sampling regions were used as 'prior' for $\mathrm{k}=1$ through $\mathrm{k}=14$. LOCPRIOR models were used because they are best suited for cases of low genetic structure (Pritchard et al. 2000). Results were evaluated using the software Structure Harvester (Earl \& VonHoldt 2012) and the appropriate number of clusters was determined by plotting the log probability $(\mathrm{L}(\mathrm{K}))$ and $\Delta \mathrm{K}$ across multiple runs according to Evanno et al. (2005). A Spearman correlation test was done using the $\mathrm{r}$ software (R Core Team 2014) to test the correlation between the proportions of clusters with latitude. A spatial Bayesian analysis was also conducted in Geneland (Guillot et al. 2012) in order to incorporate information on the spatial distribution of samples and to relate observed patterns to geography. The model was implemented in a Markov Chain Monte Carlo scheme, using 100,000 interactions, and the correlated frequency model was used, which allows the detection of structure in the presence of low genetic differentiation.

Mantel tests were calculated in GenAlEx (Peakall \& Smouse 2012) using both $F_{\text {ST }}$ and $\mathrm{R}_{\mathrm{ST}}$ and the shortest coastal distances as measured on Google Earth. Isolation by distance was first tested using all the 14 mainland populations (CHA was excluded), then excluding STE12 (due to low sample size) and then only the north and central group were used (edge populations STE12, STE14 and FIO were excluded).

To test whether genetic variation within populations is related to geographical gradients, a linear regression analysis was carried out in the software R 3.1.0 (R Core Team 2014). Latitude was plotted as a function of allelic richness $\left(A_{R}\right)$ and private allelic richness $\left(P A_{R}\right)$.

\section{Estimates of migration}

Assignment tests were used to identify first-generation migrants as well as the proportion of individuals recruiting to a location. Assignment tests are known to be more effective when genetic structure is high, therefore populations were grouped based on the four regions identified by the significance of previous analyses. The Bayesian program Geneclass 2 (Piry et al. 2004) was used with the Markov chain Monte Carlo (MCMC) resembling the algorithm of Paetkau et al. (2004). The likelihood ratio 'L_home/L_max' was used with a 0.01 rejection level and a simulated population size of 10,000 individuals per site. This approach pulls an individual's genotype from the location in which it was 
sampled over the highest likelihood observed for this genotype in any location (Paetkau et al. 2004). The individuals were assigned to the region with the highest probability.

\section{Results}

All scallops sampled were $>55 \mathrm{~mm}$ in shell length and classified as mature (Williams \& Babcock 2005). The average size of all individuals was $99.68 \pm 11.55 \mathrm{~mm}$ (Figure 3.2) and at this length they are likely to have recruited between 1.5 and 3.5 years prior to collection (2009-2011).

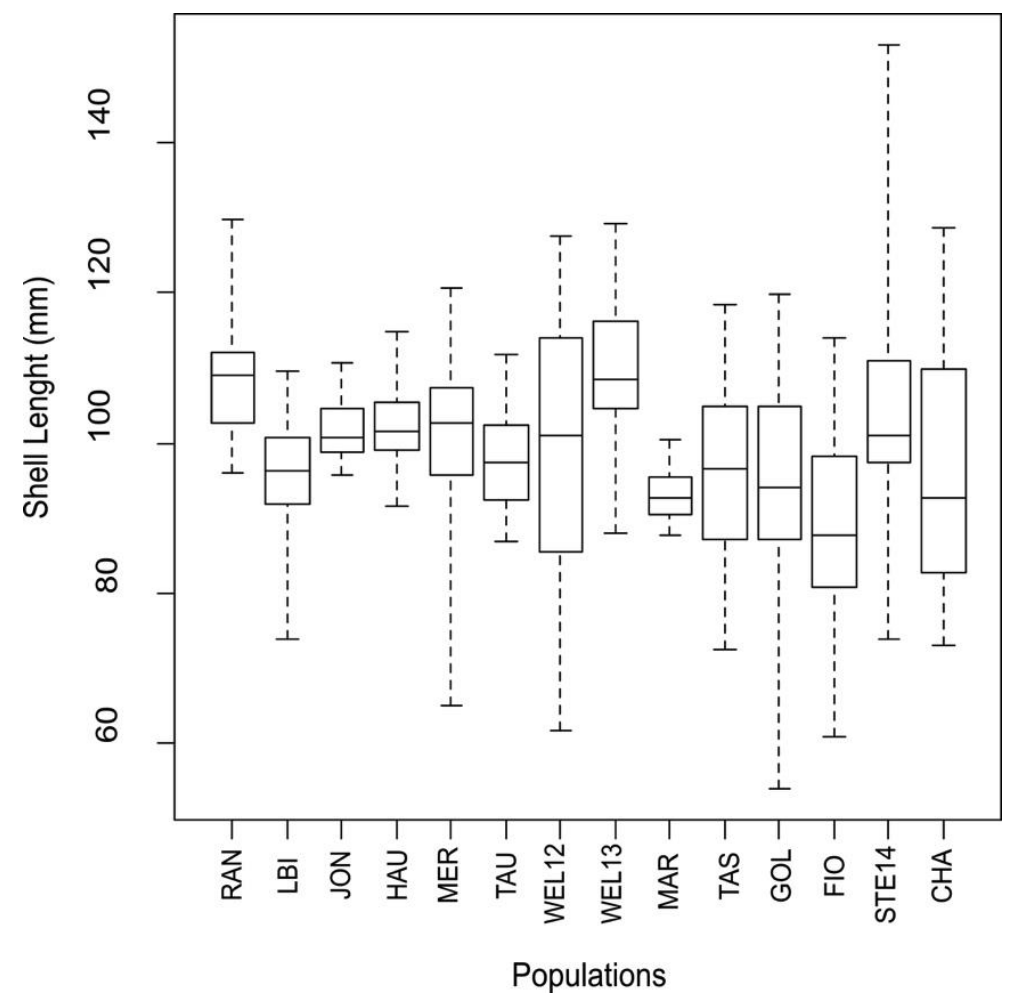

Figure 3.2 Size structure of Pecten novaezelandiae from 14 study sites in New Zealand (shells from samples collected in STE12 were not available). Dashed lines represent the maximum and minimum size at each site, while the bars represent the 25 th and 75 th quartile of size ranges, with the median indicated within each bar. 


\section{Genetic diversity}

In total, 952 individuals were genotyped for variation at 12 microsatellite loci. Pnova_24 and Pnova_31 had the most missing data at $21.3 \%$ and $19.1 \%$, respectively. The other 10 loci had an average of $2.7 \%$ of missing data.

Micro-Checker identified putative null alleles at Pnova_02, Pnova_05, Pnova_10, Pnova_24,Pnova_27 and Pnova_33 due to homozygote excess at each locus. However, no pattern or trend of null allele frequency as a function of either population or locus was identified. No long allele dropout was detected. After FDR correction for multiple tests, Pnova_24 and Pnova_33 were identified as being significantly out of HWE at more than half of the populations. No evidence of significant linkage disequilibrium was detected between locus pairs. Most of the allele discovery curves reached a plateau, with the exception of Pnova_01. The loci Pnova_27 and Pnova_28 were found to be the most polymorphic (Supplementary Figure 1).

Pnova_27 and Pnova_28 were identified as candidates for balancing selection under the stepwise mutation model and Pnova_27,Pnova_28 and Pnova_33 under the infinite alleles model. However, this is possibly an artefact due to the very low values of $F_{\mathrm{ST}}$. Pnova_01, Pnova_02, Pnova_04, Pnova_05, Pnova_09, Pnova_10 and Pnova_32 were identified as neutral markers under the infinite allele and stepwise mutation models. Pnova_24 and Pnova_31 were identified as $F_{\mathrm{ST}}$ outliers and candidates for positive selection under the infinite allele and stepwise mutation models. Pnova_24 and Pnova_31 were therefore excluded from the following analyses due to a combination of factors (i.e. missing data and $F_{\text {ST }}$ outliers).

Allelic richness ranged from 4.91 in FIO to 5.89 in RAN. Private allelic richness ranged from 0.08 in FIO to 0.26 in WEL13. In total there were 27 private alleles across all populations; RAN had the higher number (8). Overall, there were lower levels of observed heterozygosity $\mathrm{H}_{\mathrm{O}}=0.616$, than expected heterozygosity $\mathrm{H}_{\mathrm{E}}=0.659$. FIS ranged from 0.090 at STE12 to 0.146 at HAU (Table 3.2). 
Table 3.2 Descriptive measures of genetic variation in $P$. novaezelandiae for 10 loci. Allelic richness $\left(A_{R}\right)$, private allelic richness $\left(P A_{R}\right)$, number of private alleles per site $(\mathrm{Pa})$, observed heterozygosity $\left(\mathrm{H}_{\mathrm{O}}\right)$, expected heterozygosity $\left(\mathrm{H}_{\mathrm{E}}\right)$ and fixation index $\left(\mathrm{F}_{\mathrm{IS}}\right)$

\begin{tabular}{ccccccc}
\hline Population & $\mathbf{A}_{\mathbf{R}}$ & PA $_{\mathbf{R}}$ & Pa & $\mathbf{H}_{\mathbf{O}}$ & $\mathbf{H}_{\mathbf{E}}$ & $\mathbf{F}_{\mathbf{I S}}$ \\
\hline RAN & 5.89 & 0.25 & 8 & 0.616 & 0.676 & 0.078 \\
LBI & 5.83 & 0.21 & 2 & 0.605 & 0.665 & 0.088 \\
JON & 5.76 & 0.17 & 1 & 0.596 & 0.668 & 0.115 \\
HAU & 5.85 & 0.19 & 2 & 0.564 & 0.671 & 0.146 \\
MER & 5.87 & 0.21 & 5 & 0.662 & 0.670 & 0.006 \\
TAU & 5.70 & 0.19 & 1 & 0.596 & 0.658 & 0.083 \\
WEL12 & 5.73 & 0.16 & 1 & 0.639 & 0.648 & -0.001 \\
WEL13 & 5.75 & 0.26 & 1 & 0.608 & 0.651 & 0.058 \\
MAR & 5.69 & 0.16 & 0 & 0.609 & 0.661 & 0.095 \\
TAS & 5.45 & 0.12 & 1 & 0.575 & 0.642 & 0.088 \\
GOL & 5.66 & 0.11 & 0 & 0.583 & 0.641 & 0.062 \\
FIO & 4.91 & 0.08 & 0 & 0.593 & 0.635 & 0.059 \\
STE12 & 5.28 & 0.09 & 0 & 0.695 & 0.650 & -0.090 \\
STE14 & 5.41 & 0.12 & 4 & 0.689 & 0.680 & -0.013 \\
CHA & 5.50 & 0.12 & 1 & 0.611 & 0.663 & 0.074 \\
\hline
\end{tabular}

\section{Population structure}

The pairwise $F_{\mathrm{ST}}$ analysis for all comparisons ranged from 0 to 0.027 (Table 3.3). Of the 105 comparisons between pairs of populations, 44 were significant after FDR corrections. FIO, STE14 and CHA had the highest values of $F_{\mathrm{ST}}$ and therefore the highest number of statistically significant pairwise comparison outcomes. There were 6 significant comparisons between populations in the northern and central groups (WEL12-MER, WEL13-TAU, MAR-MER, MAR-TAU, TAS-HAU, TAS-MER), however there were no significant comparisons between sample sites within groups (northern and central). In terms of the mean $F_{\text {ST }}$ per population, HAU had the lowest value (0.0038) and FIO had the highest (0.0154; Table 3.3). Similar patterns were seen in values of $\mathrm{R}_{\mathrm{ST}}$, which ranged from 0 to 0.026 . However, more (45) significant $\mathrm{R}_{\mathrm{ST}}$ comparisons were observed, including one within the northern group (MER-LBI; Table 3.3). In total, $42 \%$ of the comparisons of $F_{\mathrm{ST}}$ values and $43 \%$ of $\mathrm{R}_{\mathrm{ST}}$ values were significantly different. 
The $F^{\prime}$ ST values ranged from 0 to 0.082 (Table 3.4). The populations FIO, STE12, STE14 and CHA had the highest values of $F^{\prime}$ ST when compared to other populations. A similar pattern was observed for values of $D_{\mathrm{ST}}$ that ranged from 0 to 0.060 and had the highest values for the populations FIO, STE12, STE14 and CHA (Table 3.4).

Power analysis indicated that pairwise $F_{\text {ST }}$ values as low as 0.0025 had a probability of $100 \%$ of detecting a true $F_{\mathrm{ST}}$ value for both Fisher's exact test and $\chi^{2}$. This indicates that there is sufficient resolution to detect weak differentiation among populations using 10 microsatellite loci. 
Table 3.3 Pairwise $F_{\mathrm{ST}}$ values for Pecten novaezelandiae using 10 loci are below the diagonal and $\mathrm{R}_{\mathrm{ST}}$ values are above the diagonal. Significant values are in bold after FDR testing. Mean $F_{\mathrm{ST}}$ values for each population are in the bottom row.

\begin{tabular}{|c|c|c|c|c|c|c|c|c|c|c|c|c|c|c|c|}
\hline Code & RAN & LBI & JON & HAU & MER & TAU & WEL 12 & WEL 13 & MAR & TAS & GOL & FIO & STE 12 & STE14 & CHA \\
\hline RAN & & -0.003 & -0.004 & -0.002 & 0.002 & 0.002 & 0.000 & -0.002 & -0.001 & -0.001 & 0.000 & 0.017 & 0.005 & 0.018 & 0.005 \\
\hline LBI & 0.000 & & -0.002 & 0.000 & 0.004 & 0.001 & 0.001 & 0.004 & 0.004 & -0.002 & 0.000 & 0.020 & 0.012 & 0.021 & 0.004 \\
\hline JON & 0.000 & 0.0010 & & 0.001 & -0.001 & 0.000 & -0.003 & -0.004 & -0.002 & -0.004 & 0.001 & 0.017 & 0.003 & 0.019 & 0.006 \\
\hline HAU & 0.000 & 0.001 & 0.001 & & 0.001 & 0.002 & 0.001 & 0.000 & 0.001 & 0.002 & 0.000 & 0.015 & -0.001 & 0.018 & 0.004 \\
\hline MER & 0.002 & 0.003 & 0.002 & 0.000 & & 0.000 & 0.008 & 0.006 & 0.008 & 0.007 & 0.008 & 0.016 & 0.007 & 0.026 & 0.010 \\
\hline TAU & 0.002 & 0.001 & 0.002 & 0.002 & 0.000 & & 0.005 & 0.007 & 0.009 & 0.002 & 0.004 & 0.018 & 0.008 & 0.023 & 0.008 \\
\hline WEL12 & 0.002 & 0.001 & 0.002 & 0.002 & 0.007 & 0.006 & & 0.001 & 0.003 & 0.002 & -0.002 & 0.011 & 0.007 & 0.016 & 0.003 \\
\hline WEL13 & 0.001 & 0.004 & 0.000 & 0.001 & 0.006 & 0.008 & 0.000 & & 0.003 & 0.002 & 0.002 & 0.017 & -0.002 & 0.015 & 0.007 \\
\hline MAR & 0.001 & 0.004 & 0.002 & 0.002 & 0.008 & 0.009 & 0.002 & 0.001 & & 0.002 & 0.003 & 0.011 & 0.001 & 0.016 & 0.006 \\
\hline TAS & 0.001 & 0.000 & 0.000 & 0.003 & 0.006 & 0.003 & 0.001 & 0.000 & 0.001 & & -0.002 & 0.011 & 0.003 & 0.015 & 0.005 \\
\hline GOL & 0.003 & 0.000 & 0.005 & 0.001 & 0.007 & 0.006 & 0.000 & 0.002 & 0.003 & 0.000 & & 0.009 & 0.011 & 0.017 & 0.004 \\
\hline FIO & 0.017 & 0.018 & 0.018 & 0.014 & 0.016 & 0.017 & 0.012 & 0.019 & 0.012 & 0.013 & 0.010 & & 0.016 & 0.015 & 0.019 \\
\hline STE12 & 0.005 & 0.011 & 0.004 & 0.000 & 0.009 & 0.008 & 0.011 & 0.003 & 0.004 & 0.008 & 0.015 & 0.015 & & -0.006 & 0.001 \\
\hline STE14 & 0.020 & 0.022 & 0.023 & 0.021 & 0.027 & 0.024 & 0.018 & 0.017 & 0.019 & 0.018 & 0.022 & 0.016 & 0.000 & & 0.017 \\
\hline $\mathrm{CHA}$ & 0.005 & 0.005 & 0.007 & 0.005 & 0.011 & 0.008 & 0.005 & 0.009 & 0.008 & 0.008 & 0.008 & 0.018 & 0.002 & 0.018 & \\
\hline Mean $F_{\mathrm{ST}}$ & 0.0042 & 0.0051 & 0.0048 & 0.0038 & 0.0074 & 0.0069 & 0.0049 & 0.0051 & 0.0054 & 0.0044 & 0.0059 & 0.0154 & 0.0068 & 0.0189 & 0.0084 \\
\hline
\end{tabular}


Table 3.4 $F^{\prime}$ ST values for Pecten novaezelandiae using 10 loci below diagonal and $\mathrm{D}_{\mathrm{ST}}$ values above diagonal.

\begin{tabular}{|c|c|c|c|c|c|c|c|c|c|c|c|c|c|c|c|}
\hline Code & RAN & LBI & JON & HAU & MER & TAU & WEL 12 & WEL 13 & MAR & TAS & GOL & FIO & STE 12 & STE14 & $\mathrm{CHA}$ \\
\hline JON & -0.009 & -0.002 & & 0.003 & 0.004 & 0.005 & 0.003 & 0.000 & 0.004 & 0.001 & 0.01 & 0.036 & 0.009 & 0.048 & 0.014 \\
\hline HAU & -0.007 & -0.001 & -0.004 & & 0.001 & 0.004 & 0.004 & 0.003 & 0.004 & 0.006 & 0.003 & 0.029 & -0.002 & 0.045 & 0.011 \\
\hline TAU & 0.003 & 0.000 & 0.001 & 0.003 & -0.002 & & 0.011 & 0.015 & 0.019 & 0.006 & 0.011 & 0.033 & 0.016 & 0.051 & 0.016 \\
\hline WEL12 & 0.001 & 0.000 & -0.002 & 0.001 & 0.019 & 0.013 & & 0.001 & 0.005 & 0.002 & -0.003 & 0.023 & 0.023 & 0.036 & 0.010 \\
\hline WEL13 & -0.003 & 0.008 & -0.007 & -0.001 & 0.014 & 0.018 & -0.005 & & 0.003 & 0.001 & 0.003 & 0.035 & 0.007 & 0.036 & 0.020 \\
\hline FIO & 0.045 & 0.047 & 0.045 & 0.034 & 0.042 & 0.043 & 0.029 & 0.046 & 0.027 & 0.031 & 0.02 & & 0.030 & 0.033 & 0.036 \\
\hline STE12 & -0.003 & 0.014 & -0.021 & -0.032 & 0.012 & 0.002 & 0.015 & -0.012 & -0.015 & 0.002 & 0.014 & 0.023 & & -0.001 & 0.006 \\
\hline STE14 & 0.057 & 0.063 & 0.063 & 0.058 & 0.082 & 0.069 & 0.049 & 0.048 & 0.052 & 0.051 & 0.059 & 0.044 & -0.014 & & 0.038 \\
\hline $\mathrm{CHA}$ & 0.012 & 0.014 & 0.017 & 0.014 & 0.030 & 0.021 & 0.012 & 0.026 & 0.023 & 0.023 & 0.020 & 0.050 & -0.004 & 0.053 & \\
\hline
\end{tabular}


Genetic structure was apparent from the principal components analysis (PCoA), which showed that Axis 1 explained 38\% of the variation and Axis 2 explained 25.3\% (Figure 3.3). The analysis grouped the populations sampled in the top of the North Island (RAN, LBI, JON, HAU, MER, TAU) in quadrant III and the populations sampled in central New Zealand (WEL12, WEL13, MAR, TAS, GOL) in quadrant II. Fiordland (FIO), Stewart (STE12, STE14) and Chatham Islands (CHA) were plotted separately from the remainder populations.

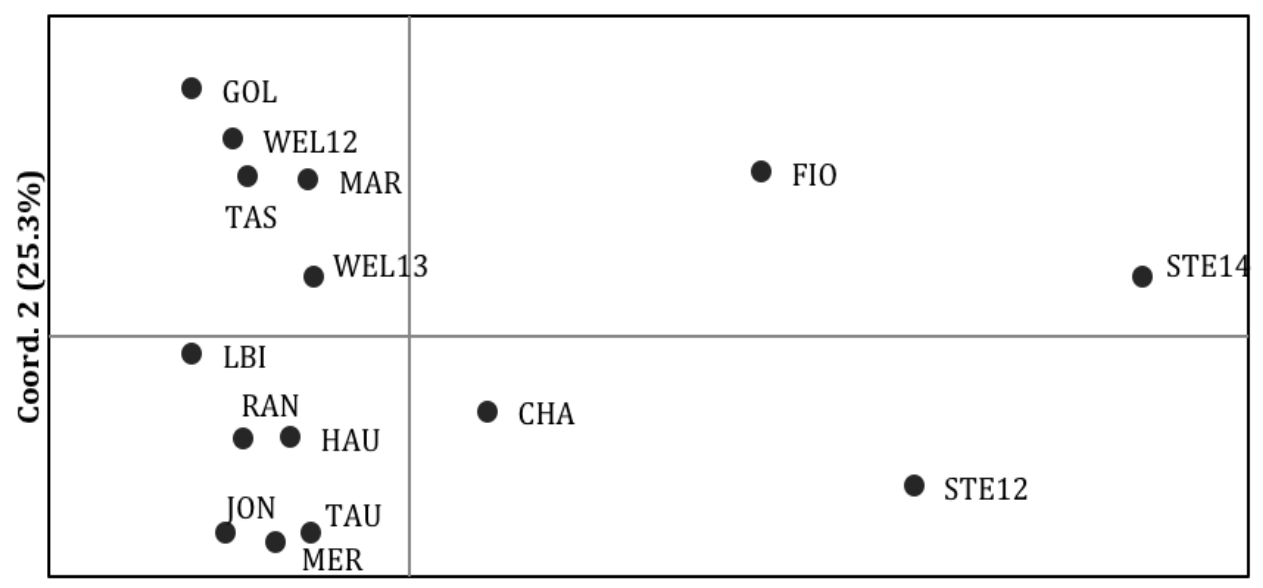

Coord. $1(38.0 \%)$

Figure 3.3 Principal components analysis (PCoA) for Pecten novaezelandiae using pairwise genetic distance based on 10 microsatellite loci.

AMOVA analysis for the four regions grouped in the PCoA analysis showed $86.2 \%$ of the variation was explained within populations, $12.8 \%$ explained among individuals, $0.3 \%$ explained among populations and $0.7 \%$ explained among regions: the model was significant at $\mathrm{p}=0.01$ (Table 3.5). Temporal differences did not explain any of the variation in the datasets of WEL12 and WEL13 populations (AMOVA, $\mathrm{p}=0.05$ ). Therefore the following analyses were conducted using WEL as one single population. 
Table 3.5 Analysis of molecular variance (AMOVA) for 14 populations from 4 regions using 10 loci.

\begin{tabular}{lllllll}
\hline $\begin{array}{l}\text { Source of } \\
\text { variation }\end{array}$ & $\begin{array}{l}\text { Degrees } \\
\text { of } \\
\text { Freedom }\end{array}$ & $\begin{array}{l}\text { Sum of } \\
\text { Squares }\end{array}$ & $\begin{array}{l}\text { Estimated } \\
\text { Variance }\end{array}$ & $\begin{array}{l}\% \\
\text { Variation }\end{array}$ & $\begin{array}{l}\text { Differentiation } \\
\text { Indexes }\end{array}$ & Significance \\
\hline $\begin{array}{l}\text { Among } \\
\text { Regions }\end{array}$ & 3 & 44.999 & 0.024 & $0.7 \%$ & $F_{\mathrm{RT}}=0.007$ & 0.010 \\
$\begin{array}{l}\text { Among } \\
\text { Populations }\end{array}$ & 10 & 52.207 & 0.011 & $0.3 \%$ & $F_{\mathrm{SR}}=0.003$ & 0.010 \\
$\begin{array}{l}\text { Among } \\
\text { Individuals }\end{array}$ & 938 & 3589.77 & 0.437 & $12.8 \%$ & $F_{\mathrm{ST}}=0.010$ & 0.010 \\
$\begin{array}{l}\text { Within } \\
\text { Populations }\end{array}$ & 952 & 2811.00 & 2.953 & $86.2 \%$ & $F_{\mathrm{IS}}=0.129$ & 0.010 \\
\hline Total & 1903 & 6497.97 & 3.425 & $100.0 \%$ & $F_{\mathrm{IT}}=0.138$ & 0.010 \\
\hline
\end{tabular}

The NJ tree showed populations STE12 and STE14 grouped together with 70\% support. The population FIO was grouped separately and CHA was grouped together with the north and central group but with low support (50\% bootstrap). All the populations from the north and central groups (RAN, LBI, JON, HAU, MER, TAU, WEL, MAR, TAS, GOL) were grouped together with $30 \%$ support. The populations GOL and TAS were grouped together with $45 \%$ support and there was high support (70\%) for the grouping of populations MER and TAU (Figure 3.4). 


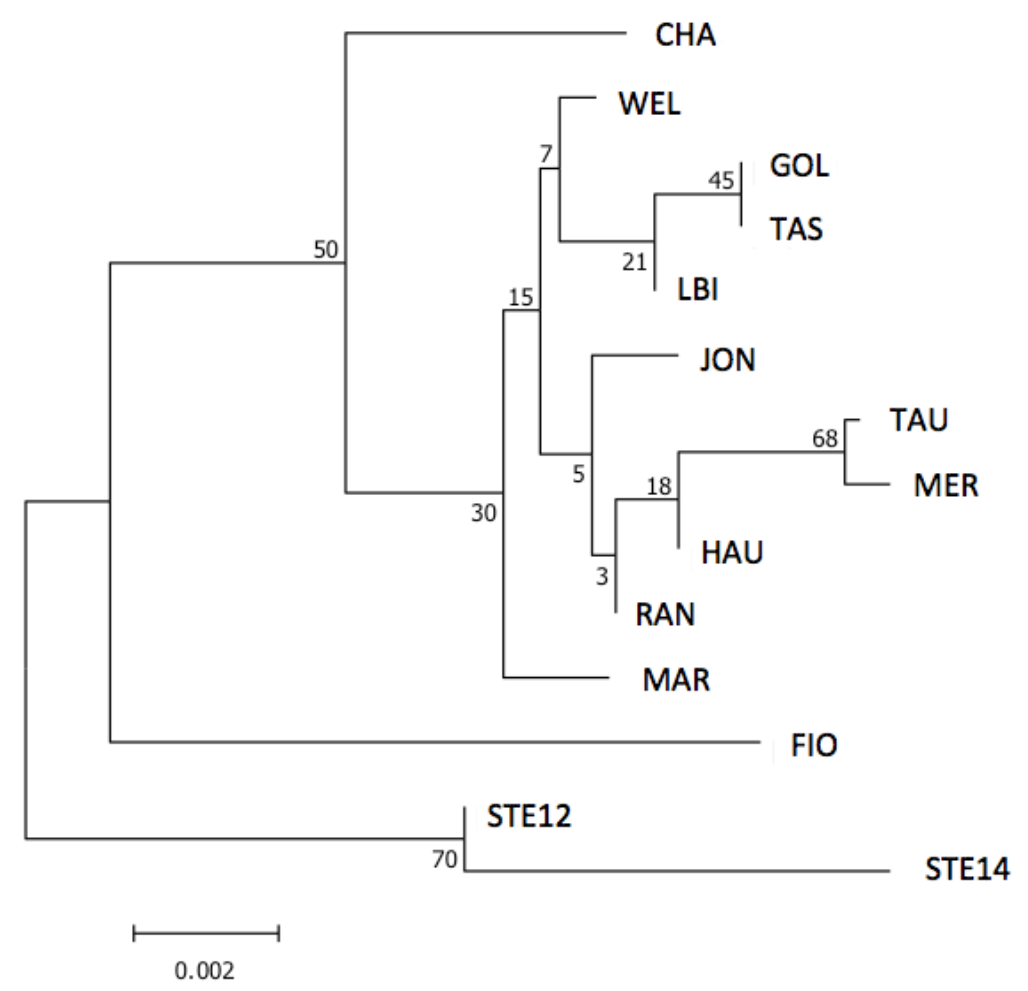

Figure 3.4 Neighbor joining population tree for Pecten novaezelandiae based on $F_{\mathrm{ST}}$ values using 14 populations.

The non-parametric (AWclust) analysis showed a large gap value at $\mathrm{k}=1$ and an increase towards $\mathrm{k}=14$ (Figure 3.5). For the Bayesian analysis (Structure) both the $\Delta \mathrm{K}$ (Evanno et al. 2005) and the maximum log-likelihood of $K$ methods showed the greatest value for 2 clusters (Table 3.6) and the bar plot showed a north-south cline of proportions of clusters (Figure 3.6). This cline was highly supported by a Spearman rank correlation test, which showed a significant decline in the proportion of Cluster 1 with increasing latitude ( $r=-$ $0.890, \mathrm{p}=0.000)$ and an increase of Cluster 2 with increasing latitude $(r=0.890, \mathrm{p}=0.000)$. 

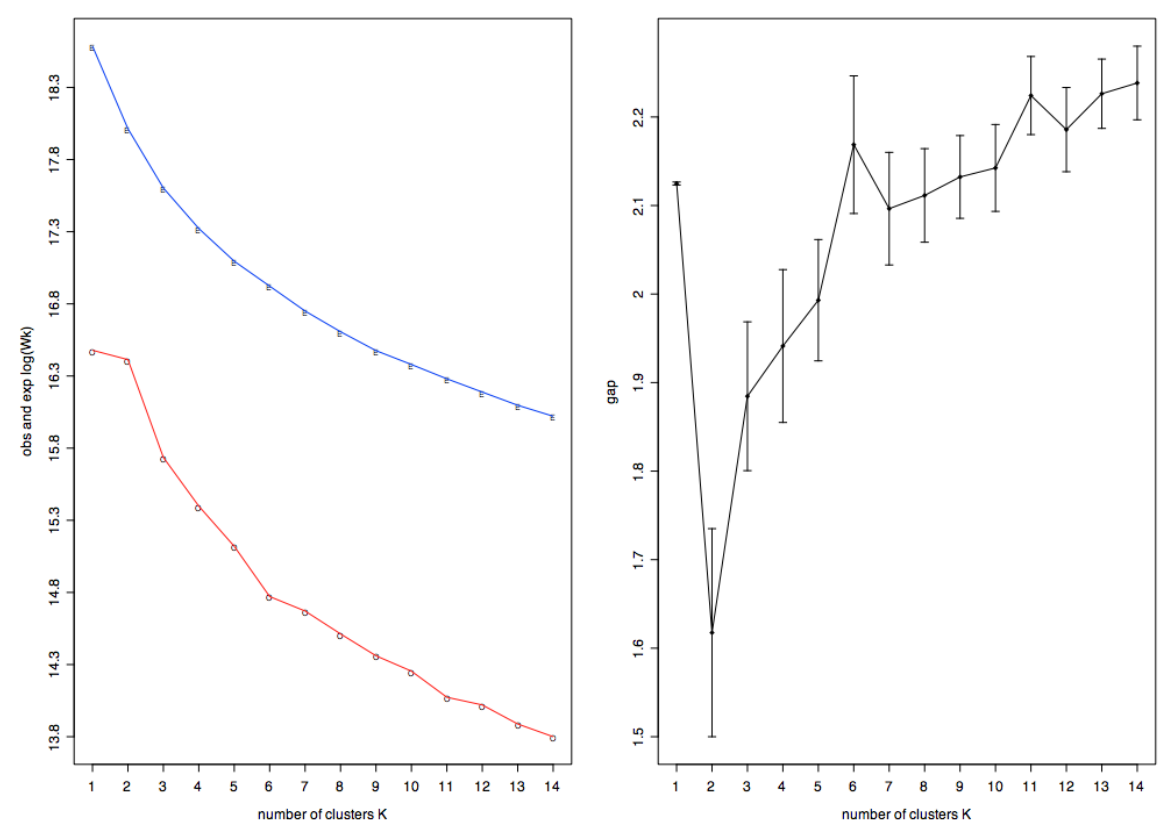

Figure 3.5 AWclust results for Pecten novaezelandiae using 10 loci: expected (E) and observed $(\mathrm{O}) \log$ pooled with-in cluster sum of squares $(\mathrm{Wk})$ (on the left) and gap statistic values between the observed and expected (on the right).

Table 3.6 Evanno table (Evanno et al. 2005) output for 14 populations from 4 regions of Pecten novaezelandiae.

$\begin{array}{ccccccc}\mathbf{K} & \text { Reps } & \text { Mean LnP(K) } & \mathbf{S t d e v} \mathbf{L n P ( K )} & \mathbf{L n}^{\prime}(\mathbf{K}) & \left|\mathbf{L n} \mathbf{n}^{\prime}(\mathbf{K})\right| & \text { Delta K } \\ 1 & 5 & -31200.08 & 0.4438 & - & - & - \\ 2 & 5 & -31069.18 & 27.740 & 130.900 & 241.36 & 8.7006 \\ 3 & 5 & -31179.64 & 171.174 & -110.460 & 264.20 & 1.5435 \\ 4 & 5 & -31554.30 & 195.677 & -374.660 & 492.92 & 2.5190 \\ 5 & 5 & -31436.04 & 169.526 & 118.260 & 637.22 & 3.7588 \\ 6 & 5 & -31955.00 & 407.973 & -518.960 & 199.40 & 0.4888 \\ 7 & 5 & -32274.56 & 1093.434 & -319.560 & 579.04 & 0.5296 \\ 8 & 5 & -32015.08 & 590.824 & 259.480 & 422.80 & 0.7156 \\ 9 & 5 & -32178.40 & 918.538 & -163.320 & 393.60 & 0.4285 \\ 10 & 5 & -31948.12 & 547.995 & 230.280 & 2425.10 & 4.4254 \\ 11 & 5 & -34142.94 & 795.644 & -2194.820 & 2853.92 & 3.5869 \\ 12 & 5 & -33483.84 & 947.134 & 659.100 & 330.36 & 0.3488 \\ 13 & 5 & -33155.10 & 552.145 & 328.740 & 934.76 & 1.6921 \\ 14 & 5 & -33761.12 & 2471.232 & -606.020 & - & -\end{array}$




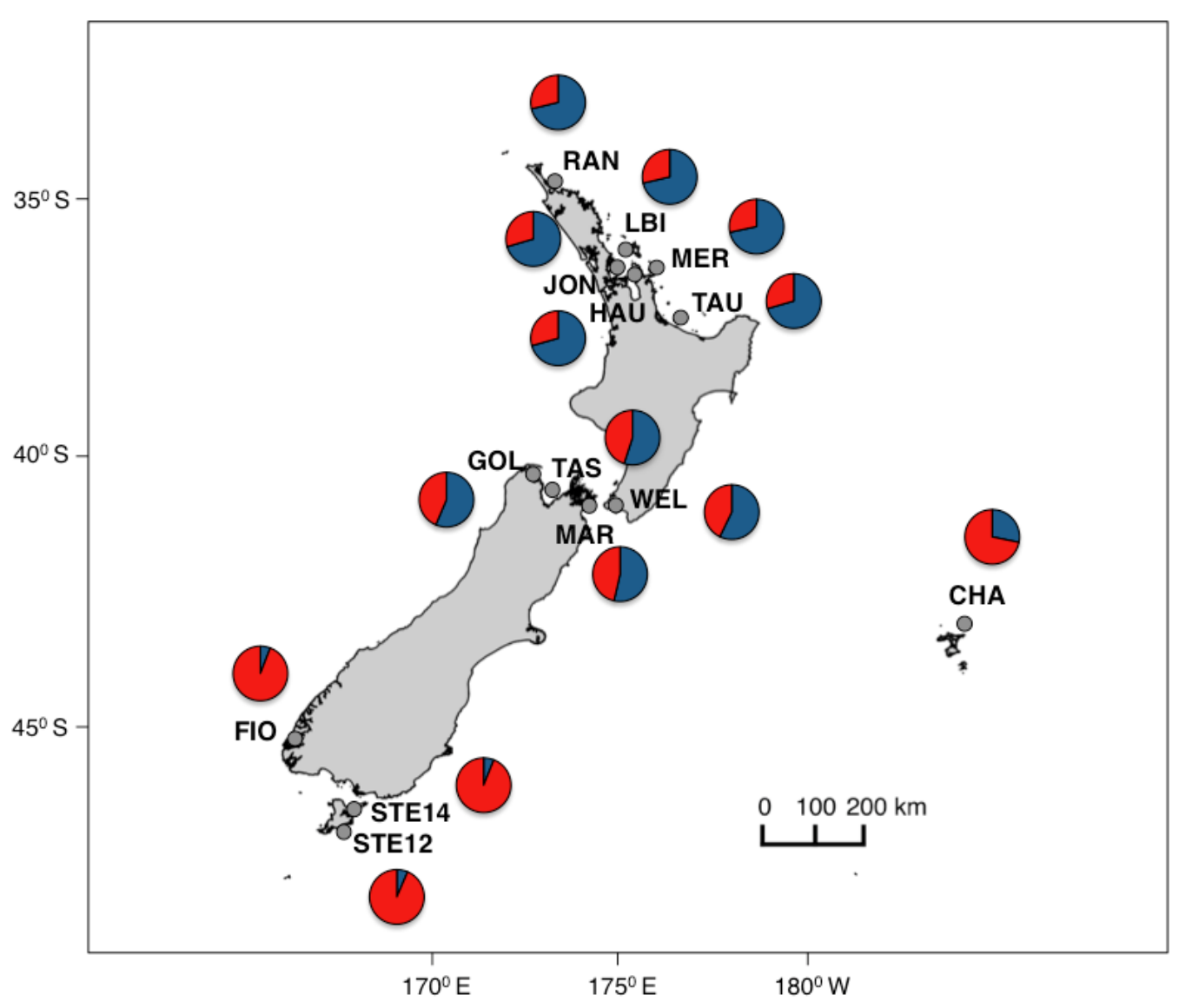

Figure 3.6 Bayesian cluster analysis (Structure) results for Pecten novaezelandiae using 10 loci $(\mathrm{K}=2)$. Samples were assigned to two colour coded-genetic clusters (blue and red).

Spatial Bayesian analysis showed that posterior distributions of $\mathrm{K}$ displayed a mode at $\mathrm{K}=5$ (Figure $3.7 \mathrm{a}, \mathrm{b}$ ). The Geneland model identified 5 spatially coherent clusters with the following groups: north (Figure 3.8b), central (Figure 3.8c), Fiordland (Figure 3.8d), Stewart Island (Figure 3.8a) and Chatham Islands (Figure 3.8e). Each cluster had a probability of $0.65,0.55,0.45,0.40$ and 0.45 of belonging to the respective group (Figure 3.8). This structure was undetected under an uncorrelated frequencies model (results not shown), possibly due to the low levels of genetic differentiation observed between populations. 
(a)

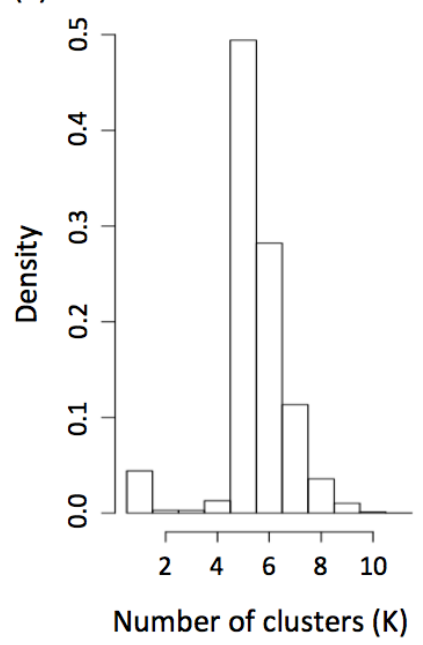

(b)

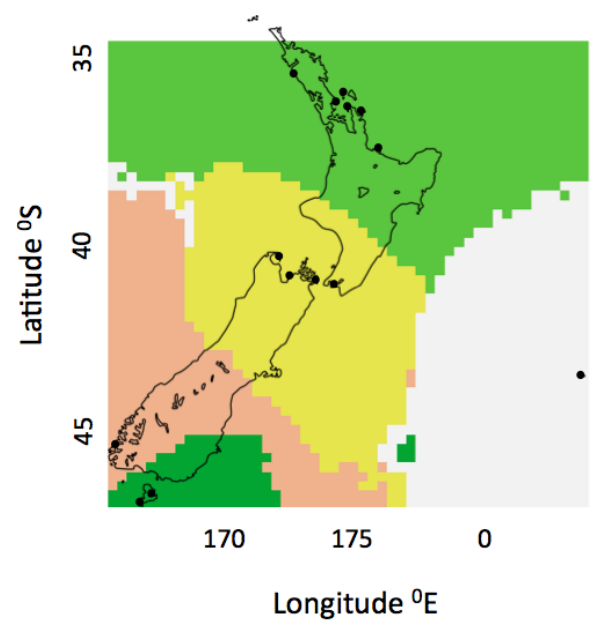

Figure 3.7 Spatial Bayesian analysis (Geneland) results for Pecten novaezelandiae using 10 loci: (a) posterior density distribution of the number of clusters $(k=5)$, (b) map of population membership for $\mathrm{k}=5$.
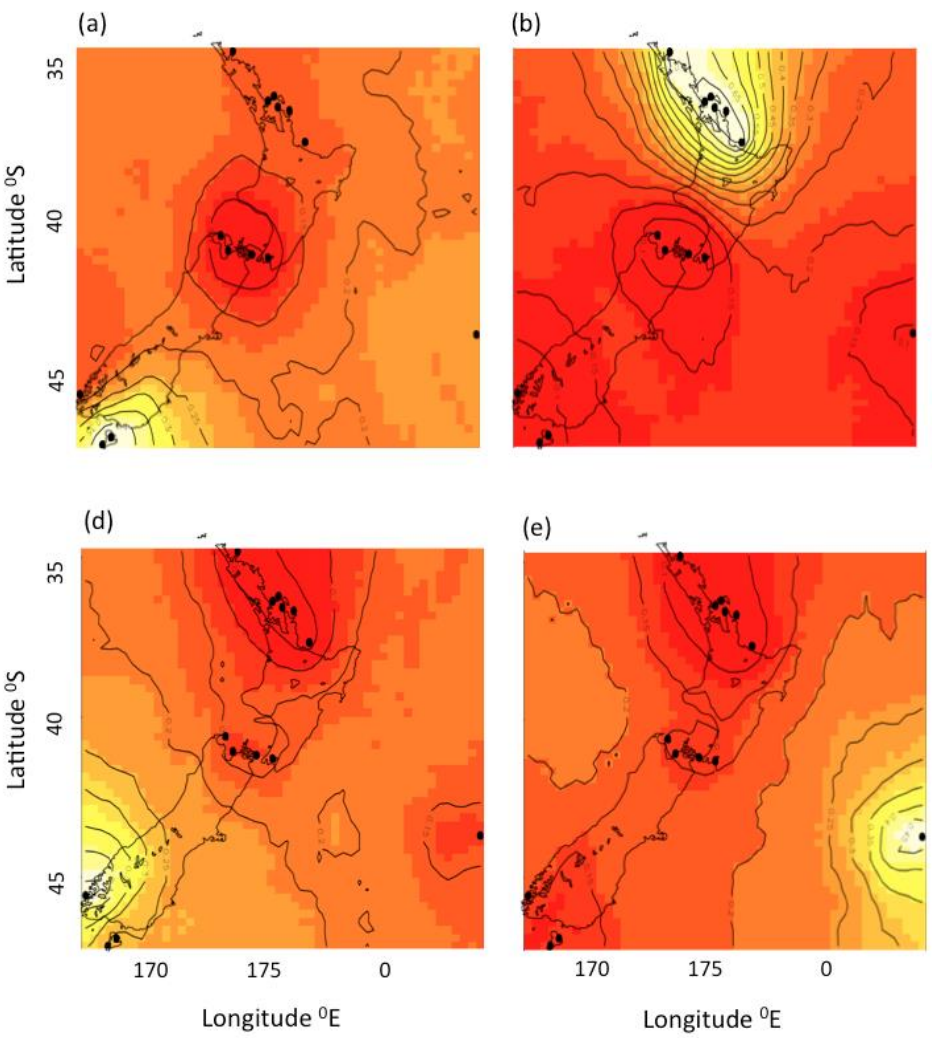

(c)

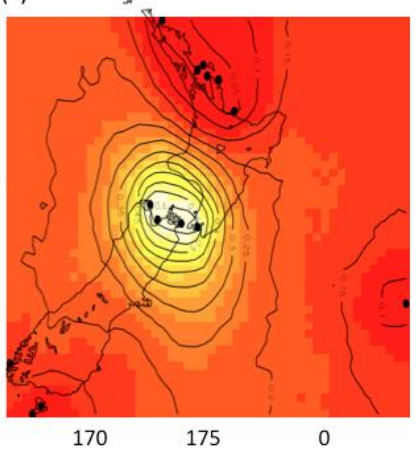

Longitude ${ }^{0} \mathrm{E}$

Figure 3.8 Spatial Bayesian analysis (Geneland) results for Pecten novaezelandiae using 10 loci: (a-e) assignment of individuals to clusters for $\mathrm{K}=1$ to $\mathrm{K}=5$, respectively. The highest population membership values are in light yellow and level curves indicate spatial changes in assignment values. 
Mantel tests showed a weak but significant isolation by distance signal when using all populations in the mainland $\left(\mathrm{R}^{2}=0.315, \mathrm{p}<0.01\right.$; Figure 3.9$)$, when excluding STE12 $\left(\mathrm{R}^{2}=0.435, \mathrm{p}<0.01\right)$ and when using only the north and central groups $\left(\mathrm{R}^{2}=0.151, \mathrm{p}=0.01\right)$. A similar trend was observed when using $\mathrm{R}_{\mathrm{ST}}$ (results not shown) excluding CHA $\left(\mathrm{R}^{2}=0.289, \mathrm{p}<0.01\right)$ and excluding STE12 $\left(\mathrm{R}^{2}=0.393, \mathrm{p}<0.01\right)$. However, when using only the north and central groups the result was not significant $\left(\mathrm{R}^{2}=0.015, \mathrm{p}=0.110\right)$.

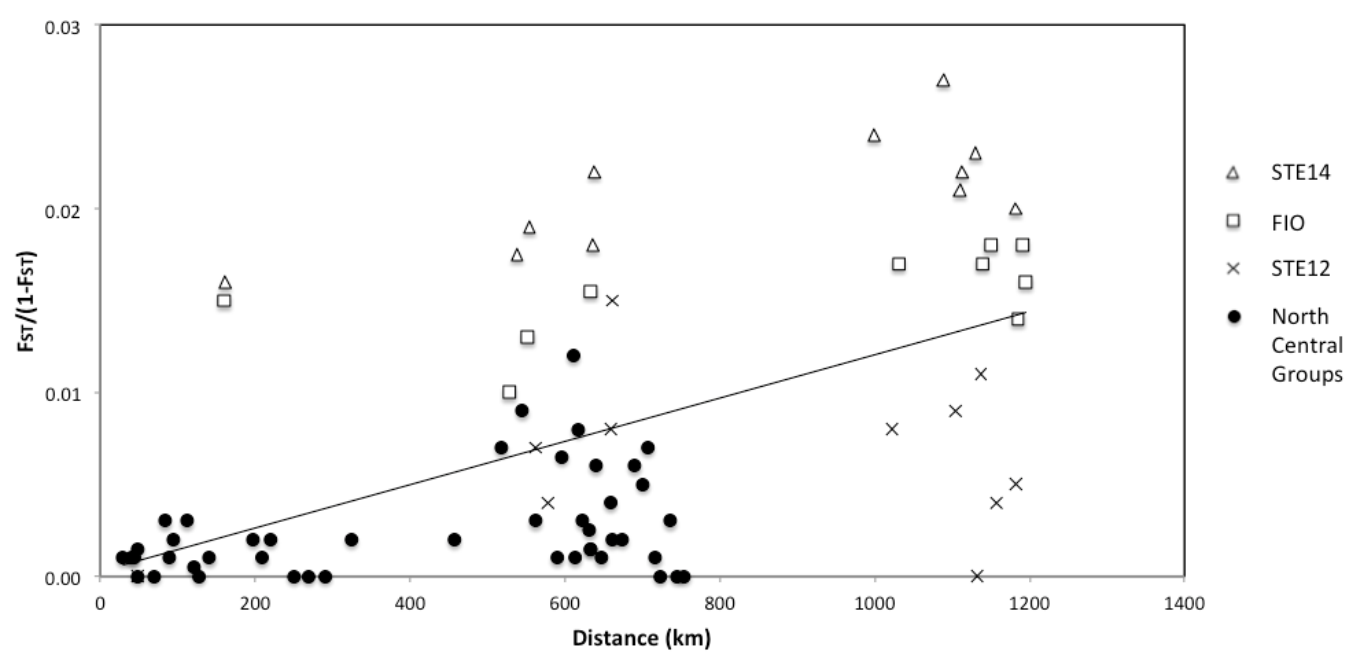

Figure 3.9 Scatterplot of Mantel tests using $F_{\mathrm{ST}}$ for all the mainland populations $\left(\mathrm{R}^{2}=0.324\right.$, $\mathrm{p}<0.01)$, when excluding STE12 $(\mathrm{n}=10) \mathrm{R}^{2}=0.461(\mathrm{p}<0.01)$ and when just the north and central groups were included $\mathrm{R}^{2}=0.148(\mathrm{p}=0.05)$. STE14: Stewart Is, Port Pegasus; FIO: Fiordland; STE12: Stewart Is, Paterson Inlet; North Central Groups: remaining populations.

Linear regression analysis showed that allelic richness $\left(A_{R}\right)$ and private allelic richness $\left(\mathrm{PA}_{\mathrm{R}}\right)$ were significantly negatively related to latitude ( $\mathrm{p}<0.001$ in both cases; Figure 3.10$)$. 

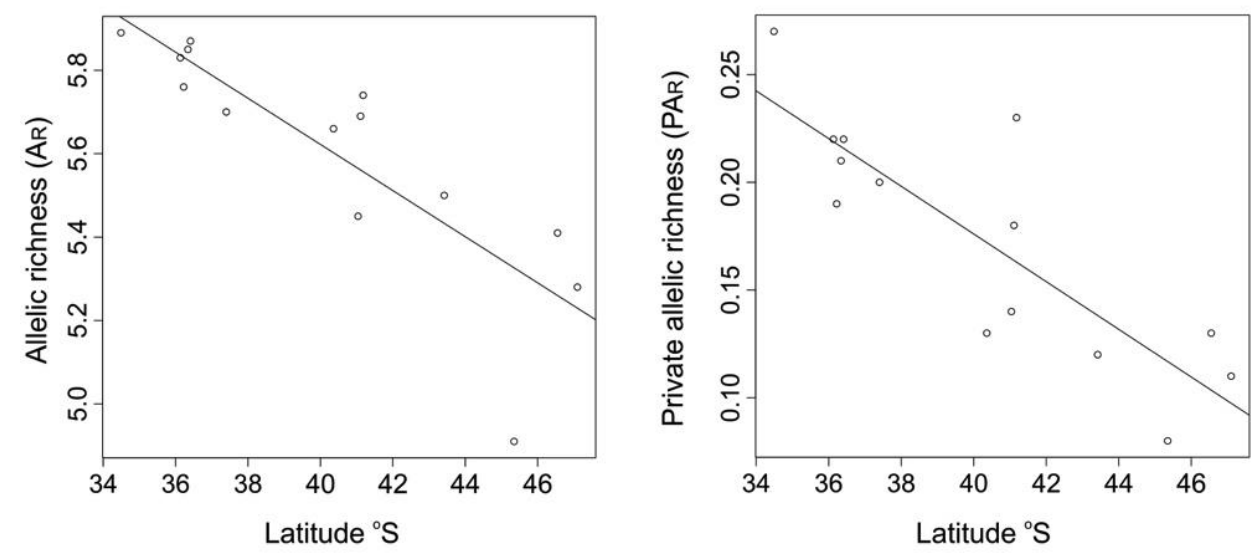

Figure 3.10 Linear regression analyses of allelic richness, $A_{R}\left(R^{2}=0.688, p=0.0001\right)$ and private allelic richness, $\mathrm{PA}_{\mathrm{R}}\left(\mathrm{R}^{2}=0.683, \mathrm{p}=0.0002\right)$ as a function of latitude.

\section{Estimates of migration}

Assignment analysis detected a low number (9) of first generation (F0) migrants for all regions (Table 3.7). Populations in the northern region were not likely to receive F0 migrants while CHA was likely to receive the most F0 migrants (4). The low number of all F0 migrants was probably an underestimation due to low levels of differentiation because migrant individuals will have very similar genotypes to the individuals from the original locality. The region with the highest level of correct assignment was the north (at 85\%), while the region with the lowest level was the Chatham Islands (CHA) at 17.3\%. There was a southwards increase in the percentage of individuals recruited from regions other than the collection site and the Chatham Islands was the region with the highest percentage at $82.7 \%$ (Table 3.8).

Table 3.7 Number of first generation (F0) migrants detected for Pecten novaezelandiae at each sampled region.

\begin{tabular}{|c|c|c|c|c|c|c|}
\hline \multicolumn{7}{|c|}{ Source of F0 migrants } \\
\hline & & North & Central & South & CHA & $\begin{array}{l}\text { Total F0 } \\
\text { migrants }\end{array}$ \\
\hline \multirow{4}{*}{ 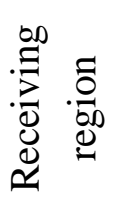 } & North & & & & & \\
\hline & Central & 2 & & & 1 & 3 \\
\hline & South & 1 & & & 1 & 2 \\
\hline & CHA & 2 & 2 & & & 4 \\
\hline
\end{tabular}


Table 3.8 Percentage of individuals of Pecten novaezelandiae collected from each sampling region, assigned to each sampled potential source region. "Total other regions" is the percentage of individuals recruited from regions other than the collection site.

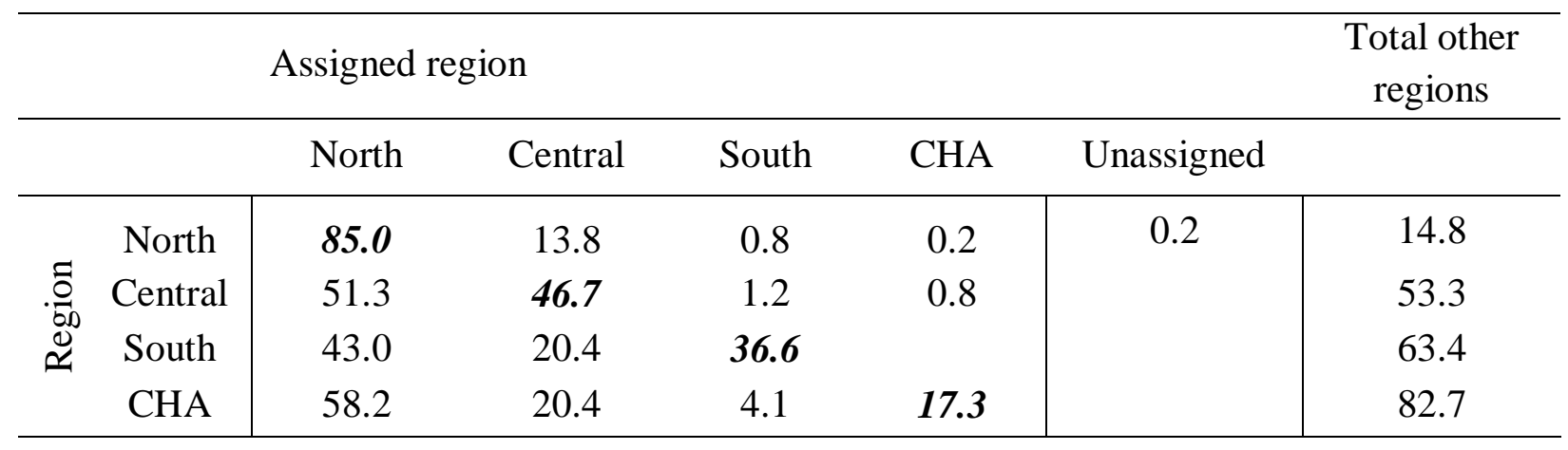

\section{Discussion}

In this chapter, the factors that shape the genetic variability and patterns of genetic structure of Pecten novaezelandiae were examined. Populations within regions appeared to be well connected with little evidence of genetic differentiation, but some level of population structure were found among regions. A significant isolation by distance signal and a degree of differentiation from north to south was apparent, but conflicted with some evidence of panmixia. The observed patterns could be associated with the geographic distance among regions, the prevailing oceanographic processes and the comparatively young evolutionary age of $P$. novaezelandiae.

\section{Population genetic structure}

Overall, population genetic differentiation was low but there were significant differences between north and central regions, and populations in the south. A multi-taxon genetic break just south of the Cook Strait at $42^{\circ} \mathrm{S}$ latitude has been observed for many coastal marine species in New Zealand (Apte \& Gardner 2002; Ayers \& Waters 2005; Goldstien et al. 2006; Ross et al. 2009; Gardner et al. 2010; Veale \& Lavery 2011). However, the populations of Pecten novaezelandiae sampled were not continuous along the coast and I was unable to obtain samples from the southern margin of the $42^{\circ} \mathrm{S}$ parallel so it was not possible to test for a similar pattern of genetic break. There may still be undetected population subdivision and a finer differentiation scale in populations that were not 
sampled. Additionally, the detection of patterns of genetic structure is dependent on the genetic markers used. Although unlikely, it is possible that the microsatellites were unable to detect a finer population structure, which could potentially be revealed by molecular markers with higher resolution such as single nucleotide polymorphisms (SNPs).

The southern region showed the highest differentiation levels from the remaining populations and higher levels of larval retention could be contributing to this structure. Chiswell \& Rickard (2011) estimated eddy diffusivity to be higher in the south of New Zealand and although the Subtropical Front flows mainly to the south turning into Cook Strait, it is weak and highly variable. Therefore, eddies in these areas may be promoting larval retention and therefore preventing larvae exchange with other populations. Also, the population in the fiords (FIO) showed the lowest levels of genetic diversity $\left(A_{R}\right.$ and $\left.P A_{R}\right)$ and high differentiation from the remaining populations. This distinct pattern of the New Zealand fiords has been observed in other studies for species with a broad distribution in New Zealand (Mladenov et al. 1997) and differentiation between fiords has also been observed elsewhere (Perrin et al. 2004) reflecting the unusual conditions of this habitat type with a semi-closed estuarine circulation.

The Chatham Islands were not very differentiated from other populations and had similarities with the mainland populations. Although the Chatham Islands are approximately $660 \mathrm{~km}$ southeast of the North Island, the subtropical convergence flowing eastward may be facilitating larval transport. This indicates that larvae of Pecten novaezelandiae have the potential to disperse over large distances and are strongly influenced by ocean currents. A similar pattern of differentiation was observed in the bivalve Austrovenus stutchburyi with similar larval duration (Ross et al. 2012) and also in the blue mussels Mytilus galloprovincialis (Westfall 2011). However, Paphies subtriangulata, also with similar pelagic larval duration shows significant differentiation from the mainland (Hannan 2014). These different patterns of genetic differentiation across multiple species likely reflect the influence of the life history and the evolutionary age of each species (Austrovenus stutchburyi - approx. 5Ma, Mytilus galloprovincialis and Pecten novaezelandiae - approx. 1Ma, Paphies subtriangulata - approx. 20Ma; Beu \& Maxwell 1990).

Fossil records in New Zealand document the origin of the genus Pecten as a result of succession of populations in the middle to late Pleistocene, approximately $1 \mathrm{Ma}$ ago (Beu 
2010). The recent evolutionary history of Pecten novaezelandiae could help explain the differences on genetic differentiation observed between $P$. novaezelandiae and other species in New Zealand. For example, the bivalve Paphies subtriangulata (Hannan 2014) is also a broadcast spawner with a similar pelagic larval duration but its older evolutionary history which dates approximately 23-16 Ma on fossil records (Fleming 1979) might help explain the stronger population structure patterns observed, particularly between the mainland and the Chatham Islands. Other species showing a genetic break at about $42^{\circ} \mathrm{S}$ latitude such Perna canaliculus and Patiriella regularis have a reported divergence time between North and South populations of approximately 1.3 Ma ago (Apte \& Gardner 2002; Ayers \& Waters 2005). On the other hand, North and South lineages of the intertidal limpets Cellana ornata, Cellana radians and Cellana flava diverged more recently, approximately 200000 to 300000 years ago (Goldstien et al. 2006). These divergence periods, correspond to stages of hydrographic change across the Cook Strait region (Lewis et al. 1994) that could have shaped the currently observed structure in some evolutionary older species in New Zealand. In addition, it is known that microsatellites can reveal the influence of both historical and contemporary processes in shaping genetic structure, for example of the North American brook trout (Pilgrim et al. 2012), and they also revealed a stepping-stone model of colonization with decline of allelic richness in the Eurasian marine invader Mnemiopsis leidyi (Reusch et al. 2010).

A loss of genetic diversity in the populations located at the edge of colonization is predicted as a consequence of the species expansion, owing to a smaller amount of genetic variation that is represented in the sink populations (Shirk et al. 2014). An increase in $F_{\mathrm{ST}}$ values towards the edges is also expected as a result of the founder events that happen as colonization advances (Gaggiotti et al. 2009). However, results showed a decrease in genetic diversity $\left(\mathrm{A}_{\mathrm{R}}\right.$ and $\left.\mathrm{PA}_{\mathrm{R}}\right)$ and an increase in differentiation ( $F_{\mathrm{ST}}$ values) towards the south. These results are in agreement with the recent evolutionary history of Pecten novaezelandiae and suggest that a possible route of colonization occurred from the north of New Zealand reflecting the species' recent expansion. In addition, the low levels of differentiation detected across the distribution range of the species might also reflect a recent range expansion and insufficient time to reach migration-drift equilibrium. Nevertheless, there is no molecular information regarding the time that lineages diverged 
and the use of mitochondrial DNA (mtDNA) could potentially add more information and increase the support in examining the phylogeographic patterns of the species.

The hierarchical genetic structure observed for $P$. novaezelandiae can also be the result of local adaptation during colonization towards the south. For example, rapid population growth and local adaptation of individuals to a new habitat can result in a successful monopolisation of resources, giving these individuals a strong priority effect (De Meester $e t$ al. 2002; Orsini et al. 2013) and these patterns of differentiation can last thousands of generations (Boileau et al. 1992). Therefore, in areas with high population densities, locally adapted individuals of $P$. novaezelandiae could be favoured over immigrants, maintaining population genetic subdivision even when levels of migration are high. Also, it has been shown for other scallop species, such as Argopecten irradians and Pecten maximus, that populations at higher latitudes spawn earlier in the season and over longer periods of time (Shumway \& Parsons 2006). This could also favour individuals locally adapted to environmental conditions over immigrants coming from northern New Zealand.

The non-parametric clustering analysis (AWclust) indicated a similar ancestor for all populations sampled $(\mathrm{k}=1)$. In contrast, the Bayesian analysis (Structure) suggested the presence of two clusters and a north-south cline of proportions of clusters. Structure analysis can be confounded by a significant signal of isolation by distance, which is known to cause Bayesian methods to overestimate genetic structure (Frantz et al. 2009). In addition, it has been shown that the earlier Structure models perform well at levels of genetic differentiation of $0.02<F_{\mathrm{ST}}<0.10$ but fail at lower values (Duchesne \& Turgeon 2012). As the highest $F_{\mathrm{ST}}$ value in this study was 0.027 and the majority of comparisons were below 0.01, it is possible that Structure failed to detect population genetic structure.

On the other hand, the spatial Bayesian analysis (Geneland) indicated the presence the 5 groups $(\mathrm{K}=5)$. The cluster probability provided strong support to the north and central clusters but the probability was below 0.5 for the groups Fiordland, Stewart Island and Chatham Islands. In general, Geneland results supported the $F_{\mathrm{ST}}$ and $\mathrm{R}_{\mathrm{ST}}$ estimates suggesting limited dispersal between these 5 groups and a low but significant genetic structure. Different methods are expected to perform better under some particular scenarios and Geneland is known to be a more sensitive approach for species with very low levels of genetic differentiation (Guillot 2008). In addition, by accounting for correlation of allele frequencies across populations this model assumes that present time populations result from 
the separation of an ancestral population which might give more clues about the on going evolutionary processes shaping the genetic structure of $P$. novaezelandiae.

Mantel tests showed a significant isolation by distance signal among samples overall and a weak but significant signal among samples in the north and central group, indicating that geographic distance may be an important factor explaining the population genetic structure of $P$. novaezelandiae. However, the Mantel test model is not a comprehensive description because sampling was not continuous along the coast but based on the hotspots of distribution of scallops. Therefore, these results might be biased, reflecting the hierarchical structure and the latitudinal genetic diversity gradient observed. The genetic signal of isolation by distance may be driven by colonization/extinction events and genetic drift during the species' recent range expansion and the genetic differentiation of the southern populations rather than by equilibrium conditions between dispersal and genetic drift.

\section{Patterns of migration}

In general, patterns of migration supported the observed differentiation between regions. Simulations suggest that the northern group persists mainly through self-recruitment. It has been shown that the pelagic larval duration can vary within scallop species depending on temperature (Beaumont \& Barnes 1992). Therefore, populations in the north of New Zealand may have a shorter pelagic larval duration and shorter potential to disperse, which could potentially explain the higher levels of self-recruitment. Estimates of migration also suggest that the northern region is a possible important source of larvae as all other regions had a high percentage of individuals that were recruited from the north group. However, it is important to consider the limitations of these results, which might also be reflecting the hierarchical genetic structure observed.

The exchange of migrants between the mainland populations and the Chatham Islands was both recent and frequent historically. Migration was detected in both directions, however over the long-term it was mainly unidirectional with a high percentage of individuals assigned to localities on the mainland (82.7\%). This substantial exchange of individuals facilitated by larval transport flowing eastward may be one of the factors explaining the low level of differentiation found between the Chatham Islands and the mainland populations. 


\section{Genetic diversity}

Levels of genetic diversity in Pecten novaezelandiae are similar to other scallop species (Pitcher et al. 2002; Kenchington et al. 2006; Zhan et al. 2009; Arias et al. 2010; Hemond \& Wilbur 2011; Marín et al. 2012) and might reflect scallops' high fecundity and ability to produce a large number of eggs resulting in large population sizes. Null alleles are frequently observed in other bivalve species (Hedgecock et al. 2004; Kenchington et al. 2006; Marín et al. 2012; Wei et al. 2013a). Similarly, they were observed for $P$. novaezelandiae, but it is unlikely that they affected the estimation of population differentiation as 1) there was no pattern or trend of null allele frequency as a function of either population or locus, 2) results from this study are consistent with other marine bivalves studies (e.g. Kenchington et al. 2006), 3) simulations suggest that $F_{\mathrm{ST}}$ values are unbiased when there is high gene flow, high effective population size $(\mathrm{Ne})$ and moderate frequencies of null alleles (Chapuis \& Estoup 2007).

The highest value of $\mathrm{F}_{\mathrm{IS}}$, observed for HAU, indicates that this is the population with the highest inbreeding rate. A possible explanation for the homozygote excess in HAU is that this population has been recently found and exploited by the fishing industry and a recent population size contraction from the resulting exploitation might have had effects on the genetic structure of this population. In contrast, the populations at Stewart Island that had the lowest values of $F_{I S}$ might be experiencing an increase in heterozygosity due to a reduction in scallop exploitation in the region. In Paterson Inlet in particular, all commercial fishing has been prohibited since 1994 and recreational fishing is more restricted than in other areas to allow scallops stocks to regenerate; fishing is permitted only in Big Glory Bay, with a limit of 10 scallops of minimum $100 \mathrm{~mm}$ shell length per diver (Carbines \& Michael 2007; Ministry of Primary Industries 2014). Interestingly, no strong evidence was found for inbreeding in the populations that recently have been described as depleted such as Tasman Bay, Golden Bay and Chatham Islands (Ministry of Primary Industries 2013b).

\section{Conclusions}

The observed geographic patterns of genetic variation of the New Zealand scallop reflect the life history of the species and the effects of ocean currents on promoting larval 
mixing/retention. Larvae of $P$. novaezelandiae have the potential for extensive dispersal and are highly influenced by changes in the prevailing ocean currents that can limit connectivity. As a consequence, patterns of genetic structure could change over time. Temporal analyses as well as the integrated use of oceanographic approaches could be particularly informative (White et al. 2010). In addition, environmental variables can also cause non-random gene flow and therefore influence patterns of genetic structure (refer to Chapter 4).

Populations in the southern margin of the scallops' distribution, such as Fiordland and Stewart Island, were significantly different, which may reflect how ecologically distinct these locations are. Since these populations have atypical genetic characteristics, they are less likely to maintain their evolutionary potential and should be managed accordingly (Johannesson \& André 2006). Furthermore, the northern region appears to be playing an important role as a source of larvae and has the highest levels of genetic diversity therefore is of particular importance for management and conservation. 


\section{CHAPTER 4}

Seascape genetics of Pecten novaezelandiae: linking environmental and genetic variation 


\section{Introduction}

Population genetic studies of marine species can be challenging because samples can be difficult to collect, neutral genetic markers can be difficult to develop, and data often do not follow traditional theoretical models (Selkoe et al. 2008). Consequently, it can be hard to identify clear geographic patterns for marine species (White et al. 2010). It has been recognized that the interactions between organisms and their environment play a crucial role in shaping spatial genetic structure. Independent of the geographic distance among populations, genetic differentiation between pairs of populations can increase with environmental differences as a result of the interactions between organisms and their environment (Wang \& Summers 2010; Wang \& Bradburd 2014). The environmental variables that might explain the genetic patterns of differentiation can be continuous, such as salinity variation, or discrete, such as sediment type; they can also describe abiotic factors such as sea surface temperature or biotic factors as chlorophyll $a$ concentration. One of the main goals of population genetic studies is therefore to understand the processes responsible for shaping the spatial genetic patterns of species. Seascape genetics is a multidisciplinary approach that supplements the genetic data with environmental variability to assess how marine environmental features may contribute to the genetic structure of organisms (Liggins et al. 2013). Complementing traditional population genetics techniques with environmental information can increase the explanatory power of population genetic studies and also elucidate about the role of environmental features in shaping the patterns of genetic structure.

Seascape genetic studies typically use neutral genetic markers to understand associations between environmental factors and neutral genetic variation (Wei et al. 2013b; D'Aloia et al. 2014). In this case, since there is no selection, the occurrence of new alleles is dependent on neutral population processes such as gene flow, genetic drift and mutation (Liggins et al. 2013). Therefore, if a particular environmental factor explains the genetic variation of a species, this environmental feature might be promoting and/or preventing gene flow among population. However, tests for selection generally have low power (Slatkin 1994, 1996), it is unclear how many studies that used genetic markers assumed to be neutral have been influenced by selection (Hudson \& Turelli 2003; Yang \& Nielsen 2008) and it is debatable whether any locus is ever absolutely neutral (Liggins et al. 2013). Nonetheless, coding 
genes under selection can obscure the processes of genetic drift and migration among populations, so neutral markers such as microsatellites are ideal for inferring demographic processes such as isolation or migration and changes in population size (Riginos \& Liggins 2013).

Organisms with a pelagic larval stage are particularly influenced by environmental variability and their dispersion and successful recruitment is highly dependent on the prevailing environmental conditions (White et al. 2010). The use of multidisciplinary tools to investigate these processes has been recognized to be particularly important for marine species, increasing the power that describes the drivers of genetic patterns in species that often exhibit a relatively weak genetic signal (Selkoe et al. 2008). Our understanding of how environmental factors affect the maintenance of genetic structure and species evolution is still very limited, and multidisciplinary studies that integrate genetics with other tools to understand which and how environmental factors influence genetic patterns are scarce. Examples include the population structure of the Atlantic herring Clupea harengus is affected by salinity at spawning sites (Gaggiotti et al. 2009), kelp cover predicts genetic structure of the three temperate reef species Paralabrax clathratus, Kelletia kelletii and Panulirus interruptus (Selkoe et al. 2010), sea surface temperature explains the genetic variation of the New Zealand greenshell mussel Perna canaliculus (Wei et al. 2013b) and environmental gradients (using chlorophyll $a$ concentration as a proxy) affect the genetic structure of the anemonefish Amphiprion bicinctus in the Red Sea (Nanninga et al. 2014).

Extending from subtropical to subantarctic waters, the New Zealand marine environment is very complex and its different habitats are characterised by a wide range of environmental factors (Shears et al. 2008). New Zealand is therefore ideal to investigate how environmental factors affect the spatial genetic patterns of marine species. However, only a limited number of seascape genetic studies have been conducted in New Zealand (e.g. Wei et al. 2013b; Constable 2014; Hannan 2014). As the endemic scallop Pecten novaezelandiae is distributed throughout New Zealand's North, South, Stewart and Chatham Islands (Shumway \& Parsons 2006), all of which encompass a wide range of environments, it is an ideal species to investigate the correlation between genetic and environmental variability. The first conducted study on the population genetic structure of the New Zealand scallop (Pecten novaezelandiae) is presented in Chapter 3. The low 
genetic signal detected across the scallop's distribution range highlights the importance of using a variety of approaches in marine population connectivity studies, especially because the statistical power to detect genetic structure in marine species can be low (Selkoe et al. 2008).

Although there is a paucity of marine environmental data for seascape genetic analyses, there has been an increase on local and global datasets that have become available to the public. To investigate the influence of environmental factors on the genetic variation of Pecten novaezelandiae, different environmental datasets were used for this study. The Marine Environmental Classification (MEC) system is an ecosystem-based spatial framework developed for marine management applications and is composed of a number of data layers with information on the New Zealand marine physical environment (Snelder $e t$ al. 2005). The benthic-optimised marine environment classification (BOMEC) scheme was specifically developed to assess the impacts of bottom trawling on benthic organisms in New Zealand (Boyer et al. 2005; Pinkerton \& Richardson 2005). Additional data layers covering a global scale now exist, some of which address gaps in the New Zealand specific data. The MODIS instrument from NASA, operating on Aqua spacecraft, views the entire surface of the Earth and acquires data every one to two days. The Ocean Color Data Processing System (OCDPS) produces and distributes the ocean colour data (Thomas \& Franz 2005).

The use of different analytical methods can increase confidence that the environmental variables are truly associated with the genetic variation. Therefore, to test the null hypothesis that none of the geospatial or environmental variables explain significant genetic variation in Pecten novaezelandiae in terms of $F_{\mathrm{ST}}$ values, $F^{\prime}$ ST values and allelic frequencies, two statistical methodologies were employed: (1) a generalised linear model (GLZ), which involves multiple regression analysis between a dependent variable and a number of predictor variables following Wei et al. (2013b) and (2) a biological environmental stepwise (BEST) analysis that tests for the relationship between resemblance matrices of dependent and predictor variables following Wei et al. (2013b). This is one of the few studies to explore the associations between New Zealand's environment features and population genetics of marine organisms (but see Wei et al. 2013b; Constable 2014; Hannan 2014). This study aimed to identify environmental variables that determine the 
population genetic structure of the New Zealand scallop Pecten novaezelandiae, bringing us one step closer to understanding the factors that shape genetic structure of populations.

\section{Materials and methods}

\section{Data collection}

Multilocus microsatellite genetic data were obtained for 952 individuals of Pecten novaezelandiae collected from 14 sites around New Zealand as described in Chapter 3 (Figure 4.1). Three geospatial variables were obtained from Google Maps for each site: (1) latitude (Lat), (2) longitude (Lon) and (3) index of geographic distance (Geo_dist) calculated as the sum of all shortest possible coastal distances $(\mathrm{km})$ between all pairs of populations (a low value indicates a population's proximity to all other populations, whereas a high value indicates its isolation, e.g. Wei et al. (2013b). Thirteen site-specific environmental variables (Table 4.1a) were obtained from the New Zealand Marine Environment Classification (MEC) scheme (New Zealand Ministry for the Environment 2005). These data are from the exclusive economic zone (EEZ) with a spatial resolution of $1 \mathrm{~km}$ and are drawn from multiple years between 1983 and 2000. The environmental variables salinity (Boyer et al. 2005), suspended particulate matter and dissolved organic matter (Pinkerton \& Richardson 2005) were obtained from the Benthic-optimised Marine Environment Classification (BOMEC) scheme (Leathwick et al. 2012; Table 4.1b). The open source Geographic Information System software QGIS 2.4 (QGIS Development Team 2014) was used to obtain site-specific data for each of the 14 locations. 


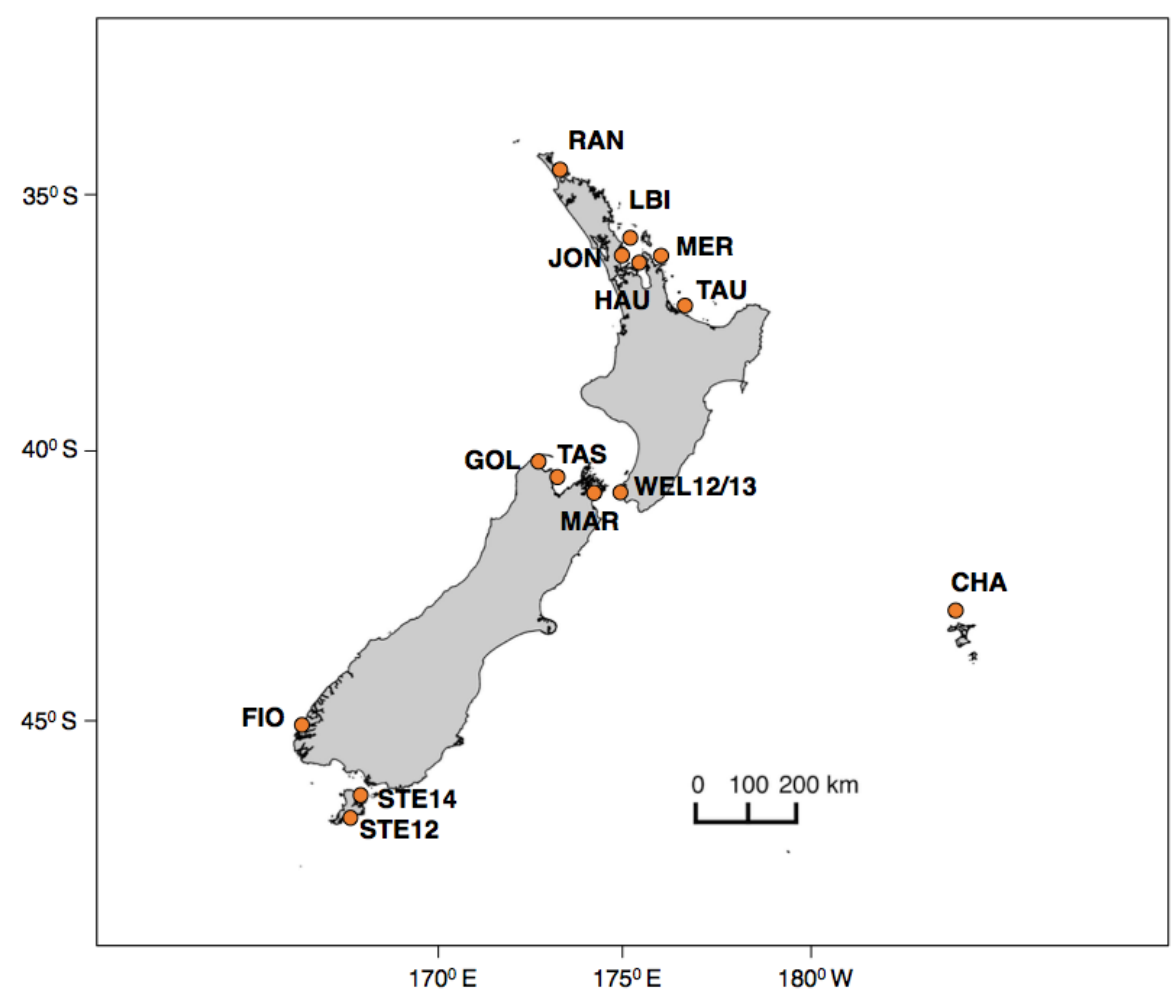

Figure 4.1 Map of sampling sites of Pecten novaezelandiae collected from New Zealand. Locations abbreviations as per Table 3.1.

The variables chlorophyll $a$ (Chl_a) and total suspended sediment (TSS) were obtained from the ocean colour satellite data MODIS project for multiple years between 2002 and 2014 (NASA 2014; Table 4.1c). Chlorophyll $a$ and total suspended sediment data were not available for the populations MAR, FIO, STE12 and CHA, which highlights that the marine environmental data available for seascape genetic studies are still limited. 
Table 4.1 Environmental variables (name), abbreviation, description and units from (a) the Marine Environment Classification (MEC), (b) the Benthic-optimised Marine Environment Classification (BOMEC) and (c) data obtained from the MODIS project.

(a)

\begin{tabular}{|c|c|c|c|}
\hline Name & Abbreviation & Description & Units \\
\hline Depth & Depth & $\begin{array}{l}\text { Bathymetry grid }(1 \mathrm{~km} \\
\text { resolution) }\end{array}$ & $\mathrm{m}$ \\
\hline $\begin{array}{l}\text { Annual mean solar } \\
\text { radiation }\end{array}$ & Rad_mean & $\begin{array}{l}\text { Mean extra atmospheric solar } \\
\text { radiation modified by mean } \\
\text { annual cloud cover }\end{array}$ & $\mathrm{Wm}^{-2}$ \\
\hline Winter solar radiation & Rad_wint & $\begin{array}{l}\text { Extra atmospheric solar radiation } \\
\text { in June, modified by mean } \\
\text { annual cloud cover }\end{array}$ & $\mathrm{Wm}^{-2}$ \\
\hline $\begin{array}{l}\text { Wintertime sea surface } \\
\text { temperature }\end{array}$ & SSTwint & $\begin{array}{l}\text { Mean of daily data from early } \\
\text { September when SST is } \\
\text { typically lowest }\end{array}$ & ${ }^{\circ} \mathrm{C}$ \\
\hline $\begin{array}{l}\text { Annual amplitude of sea } \\
\text { surface temperature }\end{array}$ & SSTanamp & $\begin{array}{l}\text { Smoothed annual amplitude of } \\
\text { SST }\end{array}$ & ${ }^{\circ} \mathrm{C}$ \\
\hline $\begin{array}{l}\text { Spatial gradient annual } \\
\text { mean sea surface } \\
\text { temperature }\end{array}$ & SSTgrad & $\begin{array}{l}\text { Smoothed magnitude of the } \\
\text { spatial gradient of annual mean } \\
\text { SST }\end{array}$ & ${ }^{\mathrm{o}} \mathrm{C} \mathrm{km}{ }^{-1}$ \\
\hline $\begin{array}{l}\text { Summertime sea surface } \\
\text { temperature anomaly }\end{array}$ & SSTanom & $\begin{array}{l}\text { Spatial anomalies with scales } \\
\text { between } 20 \text { and } 450 \mathrm{~km} \text { in late } \\
\text { February when SST is typically } \\
\text { highest }\end{array}$ & ${ }^{\circ} \mathrm{C}$ \\
\hline Mean orbital velocity & Orb_v_mean & $\begin{array}{l}\text { Orbital velocity at the bed for } \\
\text { the mean significant wave height } \\
\text { calculated from a } 20 \text {-year wave } \\
\text { hindcast }\end{array}$ & $\mathrm{m} / \mathrm{s}$ \\
\hline Extreme orbital velocity & Orb_v_95 & $\begin{array}{l}\text { Orbital velocity at the bed for } \\
\text { the } 95 \text { th percentile significant } \\
\text { wave height calculated from a } \\
20 \text {-year wave hindcast }\end{array}$ & $\mathrm{m} / \mathrm{s}$ \\
\hline Tidal current & Tidal & $\begin{array}{l}\text { Depth averaged maximum tidal } \\
\text { current }\end{array}$ & $\mathrm{m} / \mathrm{s}$ \\
\hline $\begin{array}{l}\text { Sediment type } \\
\text { (categorical variable) }\end{array}$ & Sed & $\begin{array}{l}\text { Sediment type as a categorical } \\
\text { variable }\end{array}$ & na \\
\hline $\begin{array}{l}\text { Seabed rate of change of } \\
\text { slope (profile) }\end{array}$ & Bed_prof & $\begin{array}{l}\text { The rate of change of slope for } \\
\text { each cell }\end{array}$ & $0.01 \mathrm{~m}^{-1}$ \\
\hline Freshwater fraction & FW & $\begin{array}{l}\text { Proportion of fresh water based } \\
\text { on river inputs }\end{array}$ & proportion \\
\hline Salinity & Sal & World Ocean Atlas & psu \\
\hline Dissolved organic matter & DOM & Remote sensed & $\begin{array}{l}\text { Arbitrary } \\
\text { units }\end{array}$ \\
\hline $\begin{array}{l}\text { Suspended particulate } \\
\text { matter }\end{array}$ & SPM & Remote sensed & $\begin{array}{l}\text { Arbitrary } \\
\text { units }\end{array}$ \\
\hline Chlorophyll $a$ & Chl_a & Near surface concentration & $\begin{array}{l}\mathrm{mg} \text { chl-a } \\
\mathrm{m}^{-3}\end{array}$ \\
\hline $\begin{array}{l}\text { Total suspended } \\
\text { sediment }\end{array}$ & TSS & $\begin{array}{l}\text { Total gravimetric suspended } \\
\text { sediment }\end{array}$ & $\mathrm{gm}^{-3}$ \\
\hline
\end{tabular}




\section{Correlation of variables}

The software package Statistica 12.0 was used to test for independence of the environmental variables. A Pearson correlation test and a principal component analysis (PCA) were performed and a subset of environmental variables that were correlated at the 0.05 level was removed from further analysis. The three geospatial variables and the following six independent environmental variables were used for the seascape analysis: spatial gradient annual mean sea surface temperature (SSTgrad; ${ }^{\circ} \mathrm{C} \mathrm{km}^{-1}$ ), mean orbital velocity (Orb_v_mean; $\mathrm{m} / \mathrm{s}$ ), tidal current (Tidal; $\mathrm{m} / \mathrm{s}$ ), freshwater fraction (FW; proportion), dissolved organic matter (DOM; arbitrary units) and suspended particulate matter (SPM; arbitrary units).

\section{Estimates of population genetic diversity}

Analyses were based on 952 individuals from 14 locations, as described in chapter 3 (WEL12 and WEL13 were treated as one location). Pairwise $F_{\mathrm{ST}}$ values (Weir \& Cockerham 1984) were calculated across 10 microsatellite markers using the software GenePop on the web (Rousset 2008). Modified pairwise phi-statistics ( $\left.F_{\text {ST }}^{\prime}\right)$ were calculated using the software GenoDive 2.0b25 (Meirmans \& Van Tienderen 2004; Meirmans 2006), as detailed in chapter 3. Mean multilocus $F_{\mathrm{ST}}$ and $F_{\text {ST }}^{\prime}$ values were averaged across locations following Wei et al. (2013b).

\section{Generalized Linear Model analyses}

The generalized linear model (GLZ) was calculated using the software Statistica 12.0. Akaike information criterion (AIC) was used to rank the models (best fit model with the lowest AIC score) at the significance level of $\mathrm{p}<0.05$. The analyses were run for all variables and then repeated using only geospatial or only environmental variables to determine their relative importance in explaining genetic variation, following Wei et al. (2013b). Analyses were done for all 14 locations and also excluding the 3 locations in the south, as they were outliers and it might be difficult to assess the meaningfulness of the results (FIO and STE14 were the most genetically differentiated and the sample size of STE12 was only $n=10)$. 


\section{BEST analyses}

Allele frequencies at each location were calculated using the software GenePop on the web (Rousset 2008). Following Wei et al. (2013b), to examine the association between allele frequencies and environmental/geospatial variables, the BEST routine was implemented in Primer v.6 (Clarke \& Gorley 2006). First, the analyses were run for all variables and then repeated using only geospatial or only environmental variables to determine their relative importance in explaining genetic variation. As for the GLZ, BEST analyses were done for all 14 locations and also for only 11 locations (excluding the 3 locations in the south). A Bray-Curtis resemblance matrix was employed for the allele frequencies and a Euclidean distance resemblance matrix was employed for the environmental/geospatial variables. To test for correlation between the two matrices the BIOENV routine was implemented using the Spearman correlation coefficient method $\left(\mathrm{r}_{\mathrm{s}}\right)$. Models were considered significant at $\mathrm{p}<0.05$ after 1,000 permutations. In addition, the association between allele frequencies and environmental/geospatial variables was also investigated for each locus for all 14 locations using the BEST routine to test for locusspecific responses.

\section{Results}

\section{Correlation of variables}

Pearson correlation analyses and PCA showed that the environmental variables FW, DOM and SPM were independent of all other variables. The variables Rad_mean, Rad_wint, SSTwint, SSTanamp, Sed, Sal, Chl_a and TSS were correlated with latitude whilst the variables Depth and Sed were correlated with longitude (Table 4.2; Figure 4.2). Therefore, the environmental variables SSTgrad, Orb_v_mean, Tidal, FW, DOM and SPM were used in the GLZ and BEST analyses. 
Table 4.2 Correlations between variables as defined by Pearson's correlation test. Significantly correlated variables at $\mathrm{p}<0.05$ are in bold. Abbreviations as per Table 4.1 .

\begin{tabular}{|c|c|c|c|c|c|c|c|c|c|c|c|}
\hline & Lat & Lon & Geo_dist & Depth & Rad_mean & Rad_wint & SSTwint & SSTanamp & SSTgrad & SSTanom & Orb_v_mean \\
\hline Lat & 1.000 & 0.253 & -0.525 & 0.337 & 0.969 & 0.993 & 0.953 & 0.742 & 0.006 & -0.077 & -0.422 \\
\hline Lon & 0.253 & 1.000 & -0.254 & -0.587 & 0.437 & 0.349 & 0.424 & 0.207 & 0.312 & 0.289 & 0.090 \\
\hline Geo_dist & -0.525 & -0.254 & 1.000 & -0.013 & -0.650 & -0.551 & -0.382 & -0.591 & -0.347 & 0.254 & 0.361 \\
\hline Depth & 0.337 & -0.587 & -0.013 & 1.000 & 0.163 & 0.246 & 0.182 & 0.274 & -0.213 & -0.147 & -0.425 \\
\hline Rad_mean & 0.969 & 0.437 & -0.650 & 0.163 & 1.000 & 0.987 & 0.938 & 0.761 & 0.105 & -0.053 & -0.373 \\
\hline Rad_wint & 0.993 & 0.349 & -0.551 & 0.246 & 0.987 & 1.000 & 0.962 & 0.746 & 0.038 & -0.058 & -0.401 \\
\hline SSTwint & 0.953 & 0.424 & -0.382 & 0.182 & 0.938 & 0.962 & 1.000 & 0.632 & 0.029 & 0.013 & -0.245 \\
\hline SSTanamp & 0.742 & 0.207 & -0.591 & 0.274 & 0.761 & 0.746 & 0.632 & 1.000 & -0.403 & 0.405 & -0.406 \\
\hline SSTgrad & 0.006 & 0.312 & -0.347 & -0.213 & 0.105 & 0.038 & 0.029 & -0.403 & 1.000 & -0.696 & -0.180 \\
\hline SSTanom & -0.077 & 0.289 & 0.254 & -0.148 & -0.053 & -0.058 & 0.013 & 0.405 & -0.696 & 1.000 & 0.161 \\
\hline Orb_v_mean & -0.423 & 0.090 & 0.361 & -0.425 & -0.373 & -0.401 & -0.245 & -0.406 & -0.180 & 0.161 & 1.000 \\
\hline Orb_v_95 & -0.340 & 0.110 & 0.135 & -0.499 & -0.270 & -0.313 & -0.201 & -0.330 & -0.093 & 0.030 & 0.876 \\
\hline Tidal & 0.409 & -0.029 & -0.102 & 0.169 & 0.370 & 0.414 & 0.276 & 0.585 & -0.445 & 0.191 & -0.408 \\
\hline Sed & 0.656 & 0.541 & -0.615 & -0.088 & 0.762 & 0.692 & 0.709 & 0.625 & 0.040 & 0.168 & 0.163 \\
\hline Bed_prof & 0.267 & -0.026 & -0.293 & 0.124 & 0.249 & 0.266 & 0.099 & 0.371 & 0.052 & -0.040 & -0.889 \\
\hline FW & 0.032 & 0.242 & -0.062 & -0.376 & 0.108 & 0.064 & 0.103 & -0.025 & 0.103 & 0.007 & 0.409 \\
\hline Sal & 0.972 & 0.234 & -0.380 & 0.320 & 0.922 & 0.965 & 0.966 & 0.709 & -0.096 & 0.011 & -0.356 \\
\hline DOM & 0.320 & 0.235 & -0.518 & -0.015 & 0.381 & 0.325 & 0.240 & 0.412 & 0.114 & 0.001 & -0.285 \\
\hline SPM & -0.359 & 0.125 & 0.118 & -0.310 & -0.314 & -0.341 & -0.390 & -0.162 & -0.005 & 0.211 & -0.109 \\
\hline Chl_a & -0.635 & -0.456 & 0.432 & -0.001 & -0.676 & -0.660 & -0.623 & -0.663 & 0.132 & -0.299 & 0.320 \\
\hline TSS & -0.634 & -0.456 & 0.434 & 0.007 & -0.675 & -0.659 & -0.621 & -0.658 & 0.128 & -0.290 & 0.314 \\
\hline
\end{tabular}


Table 4.2 (cont)

\begin{tabular}{|c|c|c|c|c|c|c|c|c|c|c|}
\hline & Orb_v_95 & Tidal & Sed & Bed_prof & FW & Sal & DOM & SPM & Chl_a & TSS \\
\hline Lat & -0.340 & 0.409 & 0.656 & 0.267 & 0.032 & 0.972 & 0.320 & -0.359 & -0.635 & -0.634 \\
\hline Lon & 0.110 & -0.029 & 0.541 & -0.026 & 0.242 & 0.234 & 0.235 & 0.125 & -0.456 & -0.456 \\
\hline Geo_dist & 0.135 & -0.102 & -0.615 & -0.293 & -0.062 & -0.380 & -0.518 & 0.118 & 0.432 & 0.434 \\
\hline Depth & -0.499 & 0.169 & -0.088 & 0.124 & -0.376 & 0.320 & -0.015 & -0.310 & -0.001 & 0.007 \\
\hline Rad_mean & -0.270 & 0.370 & 0.762 & 0.249 & 0.108 & 0.922 & 0.381 & -0.314 & -0.676 & -0.675 \\
\hline Rad_wint & -0.313 & 0.414 & 0.692 & 0.266 & 0.064 & 0.965 & 0.325 & -0.341 & -0.660 & -0.659 \\
\hline SSTwint & -0.201 & 0.276 & 0.709 & 0.099 & 0.103 & 0.966 & 0.240 & -0.390 & -0.623 & -0.621 \\
\hline SSTanamp & -0.330 & 0.585 & 0.625 & 0.371 & -0.025 & 0.709 & 0.412 & -0.162 & -0.663 & -0.658 \\
\hline SSTgrad & -0.093 & -0.445 & 0.040 & 0.052 & 0.103 & -0.096 & 0.114 & -0.005 & 0.132 & 0.128 \\
\hline SSTanom & 0.030 & 0.191 & 0.168 & -0.040 & 0.007 & 0.011 & 0.001 & 0.2110 & -0.299 & -0.290 \\
\hline Orb_v_mean & 0.876 & -0.408 & 0.1628 & -0.890 & 0.409 & -0.356 & -0.285 & -0.109 & 0.320 & 0.314 \\
\hline Orb_v_95 & 1.000 & -0.397 & 0.125 & -0.676 & 0.113 & -0.319 & -0.255 & -0.048 & 0.074 & 0.063 \\
\hline Tidal & -0.397 & 1.000 & 0.043 & 0.546 & -0.094 & 0.395 & 0.267 & 0.092 & -0.346 & -0.349 \\
\hline Sed & 0.125 & 0.043 & 1.000 & -0.280 & 0.464 & 0.631 & 0.407 & -0.338 & -0.437 & -0.436 \\
\hline Bed_prof & -0.676 & 0.546 & -0.280 & 1.000 & -0.458 & 0.190 & 0.261 & 0.249 & -0.360 & -0.358 \\
\hline FW & 0.113 & -0.094 & 0.464 & -0.458 & 1.000 & 0.067 & 0.198 & -0.206 & 0.383 & 0.382 \\
\hline Sal & -0.319 & 0.395 & 0.631 & 0.190 & 0.067 & 1.000 & 0.232 & -0.406 & -0.589 & -0.586 \\
\hline DOM & -0.255 & 0.267 & 0.407 & 0.261 & 0.198 & 0.232 & 1.000 & 0.212 & -0.196 & -0.197 \\
\hline SPM & -0.048 & 0.092 & -0.338 & 0.249 & -0.206 & -0.406 & 0.212 & 1.000 & -0.275 & -0.277 \\
\hline Chl_a & 0.074 & -0.346 & -0.437 & -0.360 & 0.383 & -0.589 & -0.196 & -0.275 & 1.000 & 0.999 \\
\hline TSS & 0.063 & -0.349 & -0.436 & -0.358 & 0.382 & -0.586 & -0.197 & -0.277 & 0.999 & 1.000 \\
\hline
\end{tabular}




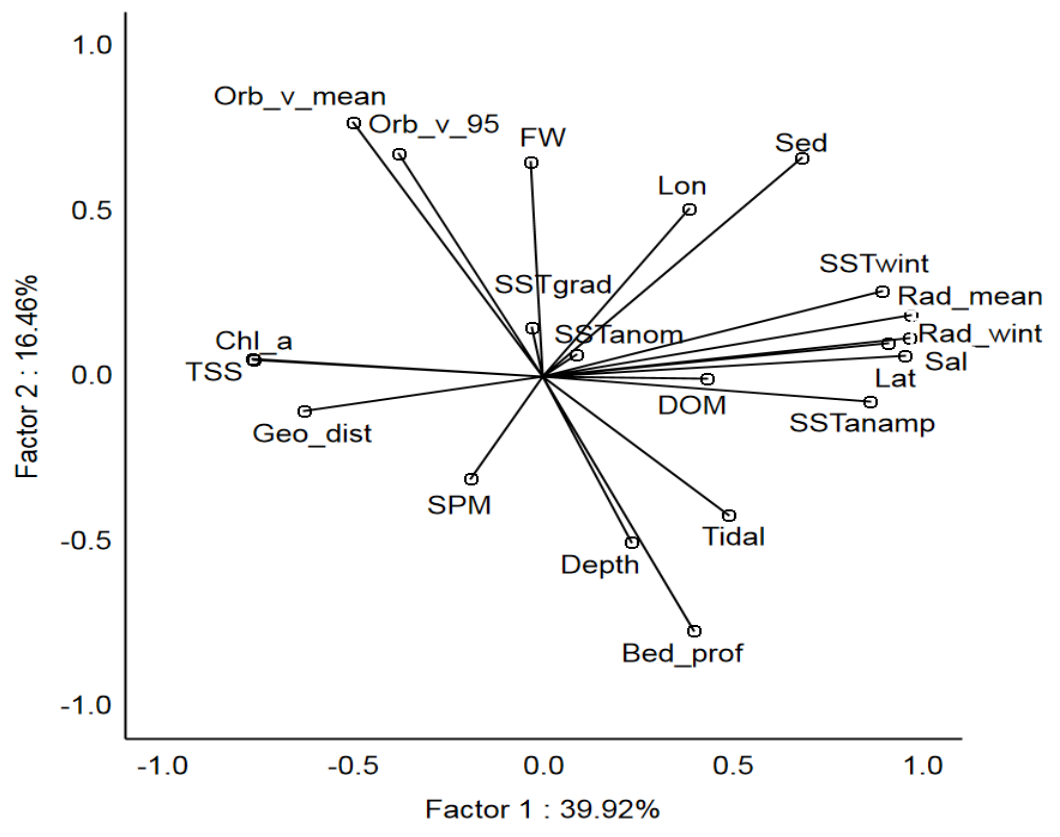

Figure 4.2 Principal component analysis (PCA) of both the geospatial and environmental variables. Abbreviations as per Table 4.1.

\section{Generalized Linear Models}

The pairwise $F_{\text {ST }}$ values for all comparisons ranged from 0 to 0.027 while the $F^{\prime}$ ST values ranged from 0 to 0.082 . Mean $F_{\text {ST }}$ values ranged from 0.004 at HAU to 0.019 at STE14 and mean $F^{\prime}$ ST values ranged from -0.001 at STE12 to 0.052 at STE14.

Generalized linear models based on data from all 14 locations showed that the top ten best fitting models were all significant at $\mathrm{p}<0.01$ when testing variation in both $F_{\text {ST }}$ and $F^{\prime}$ ST values against variation in all 9 geospatial and environmental variables. All the variables were included in the models, however only the variables Lon, Geo_dist, Tidal, FW and SPM were significant at $\mathrm{p}<0.05$ for the test of all effects (Table 4.3).

Generalized linear model analyses based on data from 11 locations (excluding south) showed that the top 9 best fitting models were significant at $\mathrm{p}<0.05$ when testing variation in $F_{\mathrm{ST}}$ values against variation in all 9 geospatial and environmental variables. All variables were included in these models but only Lon appeared in all the models and Tidal was significant at $\mathrm{p}<0.05$ for the test of all effects. When testing variation in $F^{\prime}$ ST values against variation in all 9 geospatial and environmental variables, the top ten best fitting models 
were all significant at $\mathrm{p}<0.05$, all the variables were included in the models and all were significant at $\mathrm{p}<0.05$ for the test of all effects (Table 4.3).

Table 4.3 Results of the top ten best fitting models of generalized linear model analyses (GLZ routine in STATISTICA v12) testing for the contribution of all 9 geospatial and environmental variables to explain variation in $F_{\mathrm{ST}}$ and $F_{\text {ST }}^{\prime}$ for Pecten novaezelandiae. The checkmarks $(\checkmark)$ indicate which variables were included in each model. ${ }^{1}$ p-value of each model; ${ }^{2} \mathrm{p}$-value of the test of all effects, significant values at $\mathrm{p}<0.05$ are in bold.

\begin{tabular}{|c|c|c|c|c|c|c|c|c|c|}
\hline \multicolumn{10}{|c|}{$F_{\mathrm{ST}}$ based on all 14 locations } \\
\hline p-value ${ }^{1}$ & Lat & Lon & $\begin{array}{c}\mathrm{Geo}_{-} \\
\text {dist }\end{array}$ & $\begin{array}{l}\text { SST } \\
\text { grad }\end{array}$ & $\begin{array}{c}\text { Orb_v } \\
\text { mean }\end{array}$ & Tidal & FW & DOM & SPM \\
\hline$<0.001$ & & $\checkmark$ & $\checkmark$ & & & $\checkmark$ & $\checkmark$ & & $\checkmark$ \\
\hline$<0.001$ & & $\checkmark$ & $\checkmark$ & & & $\checkmark$ & $\checkmark$ & $\checkmark$ & $\checkmark$ \\
\hline$<0.001$ & & $\checkmark$ & $\checkmark$ & $\checkmark$ & & $\checkmark$ & $\checkmark$ & & $\checkmark$ \\
\hline$<0.001$ & & $\checkmark$ & $\checkmark$ & & $\checkmark$ & $\checkmark$ & $\checkmark$ & & $\checkmark$ \\
\hline$<0.001$ & & $\checkmark$ & $\checkmark$ & $\checkmark$ & & $\checkmark$ & $\checkmark$ & $\checkmark$ & $\checkmark$ \\
\hline$<0.001$ & & $\checkmark$ & $\checkmark$ & & $\checkmark$ & $\checkmark$ & $\checkmark$ & $\checkmark$ & $\checkmark$ \\
\hline$<0.001$ & & & $\checkmark$ & $\checkmark$ & & $\checkmark$ & $\checkmark$ & & $\checkmark$ \\
\hline$<0.001$ & & & $\checkmark$ & & & $\checkmark$ & $\checkmark$ & & $\checkmark$ \\
\hline$<0.001$ & $\checkmark$ & $\checkmark$ & $\checkmark$ & & & $\checkmark$ & $\checkmark$ & & $\checkmark$ \\
\hline$<0.001$ & & $\checkmark$ & $\checkmark$ & $\checkmark$ & $\checkmark$ & $\checkmark$ & $\checkmark$ & & $\checkmark$ \\
\hline p-value ${ }^{2}$ & 0.824 & 0.065 & 0.004 & 0.339 & 0.429 & 0.029 & $<0.001$ & 0.183 & $<0.001$ \\
\hline \multicolumn{10}{|c|}{$F_{\text {ST }}^{\prime}$ based on all 14 locations } \\
\hline p-value ${ }^{1}$ & Lat & Lon & $\begin{array}{c}\mathrm{GeO}_{-} \\
\text {dist }\end{array}$ & $\begin{array}{l}\text { SST } \\
\text { grad }\end{array}$ & $\begin{array}{c}\text { Orb_v__ } \\
\text { mean }\end{array}$ & Tidal & FW & DOM & SPM \\
\hline$<0.001$ & & $\checkmark$ & & & & & $\checkmark$ & $\checkmark$ & $\checkmark$ \\
\hline$<0.001$ & $\checkmark$ & $\checkmark$ & & & & & $\checkmark$ & $\checkmark$ & $\checkmark$ \\
\hline$<0.001$ & $\checkmark$ & $\checkmark$ & & & & $\checkmark$ & $\checkmark$ & $\checkmark$ & $\checkmark$ \\
\hline$<0.001$ & & $\checkmark$ & & & & $\checkmark$ & $\checkmark$ & $\checkmark$ & $\checkmark$ \\
\hline$<0.001$ & & $\checkmark$ & $\checkmark$ & & & & $\checkmark$ & $\checkmark$ & $\checkmark$ \\
\hline$<0.001$ & $\checkmark$ & $\checkmark$ & & $\checkmark$ & & $\checkmark$ & $\checkmark$ & $\checkmark$ & $\checkmark$ \\
\hline$<0.001$ & & $\checkmark$ & & $\checkmark$ & & & $\checkmark$ & $\checkmark$ & $\checkmark$ \\
\hline$<0.001$ & & $\checkmark$ & & & $\checkmark$ & & $\checkmark$ & $\checkmark$ & $\checkmark$ \\
\hline$<0.001$ & & $\checkmark$ & & $\checkmark$ & & $\checkmark$ & $\checkmark$ & $\checkmark$ & $\checkmark$ \\
\hline$<0.001$ & $\checkmark$ & $\checkmark$ & $\checkmark$ & & & & $\checkmark$ & $\checkmark$ & $\checkmark$ \\
\hline p-value ${ }^{2}$ & 0.136 & 0.014 & 0.491 & 0.243 & 0.410 & 0.025 & 0.016 & 0.085 & 0.001 \\
\hline
\end{tabular}


Table 4.3 (Cont.)

\begin{tabular}{|c|c|c|c|c|c|c|c|c|c|}
\hline \multicolumn{10}{|c|}{$F_{\mathrm{ST}}$ based on 11 locations (Excluding south) } \\
\hline p-value ${ }^{1}$ & Lat & Lon & $\begin{array}{c}\mathrm{Geo}_{-} \\
\text {dist }\end{array}$ & $\begin{array}{l}\text { SST } \\
\text { grad }\end{array}$ & $\begin{array}{c}\text { Orb_v_- } \\
\text { mean }\end{array}$ & Tidal & FW & DOM & SPM \\
\hline 0.014 & & $\checkmark$ & & & & & & & \\
\hline 0.032 & & $\checkmark$ & & & & $\checkmark$ & & & \\
\hline 0.039 & & $\checkmark$ & & & & & & $\checkmark$ & \\
\hline 0.042 & & $\checkmark$ & & & & & & & $\checkmark$ \\
\hline 0.043 & & $\checkmark$ & & $\checkmark$ & & & & & \\
\hline 0.044 & & $\checkmark$ & & & & & $\checkmark$ & & \\
\hline 0.045 & & $\checkmark$ & & & $\checkmark$ & & & & \\
\hline 0.048 & & $\checkmark$ & $\checkmark$ & & & & & & \\
\hline 0.049 & $\checkmark$ & $\checkmark$ & & & & & & & \\
\hline 0.051 & & $\checkmark$ & & & & $\checkmark$ & & $\checkmark$ & \\
\hline p-value ${ }^{2}$ & 0.083 & 0.059 & 0.088 & 0.107 & 0.393 & $\mathbf{0 . 0 3 5}$ & 0.920 & 0.843 & 0.461 \\
\hline \multicolumn{10}{|c|}{$F_{\text {ST }}^{\prime}$ based on 11 locations (Excluding south) } \\
\hline p-value ${ }^{1}$ & Lat & Lon & $\begin{array}{c}\mathrm{Geo}_{-} \\
\text {dist }\end{array}$ & $\begin{array}{l}\text { SST } \\
\text { grad }\end{array}$ & $\begin{array}{c}\text { Orb_v } \\
\text { mean }\end{array}$ & Tidal & FW & DOM & SPM \\
\hline 0.002 & $\checkmark$ & $\checkmark$ & $\checkmark$ & $\checkmark$ & $\checkmark$ & $\checkmark$ & $\checkmark$ & $\checkmark$ & $\checkmark$ \\
\hline 0.007 & & $\checkmark$ & & & & & & & \\
\hline 0.015 & & $\checkmark$ & & & & $\checkmark$ & & & \\
\hline 0.023 & & $\checkmark$ & & $\checkmark$ & & & & & \\
\hline 0.023 & & $\checkmark$ & & & & & & & $\checkmark$ \\
\hline 0.025 & & $\checkmark$ & & & & & & $\checkmark$ & \\
\hline 0.027 & $\checkmark$ & $\checkmark$ & & & & & & & \\
\hline 0.027 & & $\checkmark$ & & & & & $\checkmark$ & & \\
\hline 0.027 & & $\checkmark$ & & & $\checkmark$ & & & & \\
\hline 0.028 & & $\checkmark$ & $\checkmark$ & & & & & & \\
\hline p-value ${ }^{2}$ & $<0.001$ & $<0.001$ & $<0.001$ & $<0.001$ & $<0.001$ & $<0.001$ & $<0.001$ & $<0.001$ & $<0.001$ \\
\hline
\end{tabular}

When generalized linear models were run for only 3 geospatial variables based on data from all 14 locations, the top six best fitting models were significant at $p<0.01$ when testing variation in $F_{\text {ST }}$ values and included all the geospatial variables. When testing variation in $F^{\prime}$ ST values against variation in geospatial variables, the top two best fitting models were significant at $\mathrm{p}<0.05$ and included only the variables Lat and Geo_dist. Generalized linear model analyses based on data from 11 locations showed that the top three best fitting models were significant at $\mathrm{p}<0.05$ when testing variation in $F_{\mathrm{ST}}$ values against variation in geospatial variables and included all geospatial variables. When testing variation in $F^{\prime}$ ST values against variation in geospatial variables, the top five best fitting models were 
significant at $\mathrm{p}<0.05$ and also included all geospatial variables. However, none of the geospatial variables were significant for the test of all effects for any scenario (Table 4.4).

Table 4.4 Results of the top ten best fitting models of generalized linear model analyses (GLZ routine in STATISTICA v12) testing for the contribution of 3 geospatial variables to explain variation in $F_{\mathrm{ST}}$ and $F_{\text {ST }}^{\prime}$ for Pecten novaezelandiae. The checkmarks $(\checkmark)$ indicate which variables were included in each model. ${ }^{1} \mathrm{p}$-value of each model; ${ }^{2} \mathrm{p}$-value of the test of all effects.

\begin{tabular}{|c|c|c|c|c|c|c|c|}
\hline \multicolumn{4}{|c|}{14 locations } & \multicolumn{4}{|c|}{11 locations (Excluding south) } \\
\hline \multicolumn{4}{|c|}{$F_{\mathrm{ST}}$} & \multicolumn{4}{|c|}{$F_{\mathrm{ST}}$} \\
\hline p-value ${ }^{1}$ & Lat & Lon & Geo_dist & p-value ${ }^{1}$ & Lat & Lon & Geo_dist \\
\hline 0.002 & $\checkmark$ & & $\checkmark$ & 0.014 & & $\checkmark$ & \\
\hline 0.001 & & & $\checkmark$ & 0.048 & & $\checkmark$ & $\checkmark$ \\
\hline 0.002 & $\checkmark$ & & & 0.049 & $\checkmark$ & $\checkmark$ & \\
\hline 0.004 & $\checkmark$ & $\checkmark$ & $\checkmark$ & 0.061 & $\checkmark$ & & $\checkmark$ \\
\hline 0.005 & & $\checkmark$ & $\checkmark$ & 0.096 & $\checkmark$ & $\checkmark$ & $\checkmark$ \\
\hline 0.007 & $\checkmark$ & $\checkmark$ & & 0.145 & $\checkmark$ & & \\
\hline 0.728 & & $\checkmark$ & & 0.251 & & & $\checkmark$ \\
\hline p-value ${ }^{2}$ & 0.125 & 0.639 & 0.091 & p-value ${ }^{2}$ & 0.614 & 0.389 & 0.575 \\
\hline \multicolumn{4}{|c|}{$F_{\mathrm{ST}}^{\prime}$} & \multicolumn{4}{|c|}{$F_{\mathrm{ST}}^{\prime}$} \\
\hline p-value ${ }^{1}$ & Lat & Lon & Geo_dist & p-value ${ }^{1}$ & Lat & Lon & Geo_dist \\
\hline 0.021 & & & $\checkmark$ & 0.007 & & $\checkmark$ & \\
\hline 0.036 & $\checkmark$ & & & 0.027 & $\checkmark$ & $\checkmark$ & \\
\hline 0.057 & $\checkmark$ & & $\checkmark$ & 0.028 & & $\checkmark$ & $\checkmark$ \\
\hline 0.067 & & $\checkmark$ & $\checkmark$ & 0.037 & $\checkmark$ & & $\checkmark$ \\
\hline 0.106 & $\checkmark$ & $\checkmark$ & & 0.041 & $\checkmark$ & $\checkmark$ & $\checkmark$ \\
\hline 0.123 & $\checkmark$ & $\checkmark$ & $\checkmark$ & 0.095 & & & $\checkmark$ \\
\hline 0.526 & & $\checkmark$ & & 0.197 & $\checkmark$ & & \\
\hline p-value ${ }^{2}$ & 0.558 & 0.809 & 0.313 & p-value ${ }^{2}$ & 0.314 & 0.209 & 0.323 \\
\hline
\end{tabular}

When generalized linear models were run for only 6 environmental variables based on data from all 14 locations, the top ten best fitting models were all significant at $p<0.01$ when testing variation in $F_{\mathrm{ST}}$ values against variation in environmental variables. All the environmental variables were included in the models, but only Tidal, FW, DOM and SPM were significant at $\mathrm{p}<0.05$ for the test of all effects. The top ten best fitting models were also all significant at $\mathrm{p}<0.01$ when testing variation in $F^{\prime}$ ST values against variation in 
environmenal variables. All the environmental variables were included in the models, but only SPM was significant at $\mathrm{p}<0.05$ for the test of all effects. Generalized linear model analyses based on data from 11 locations showed that none of the models were significant when testing variation in both $F_{\mathrm{ST}}$ and $F_{\text {ST }}^{\prime}$ values against variation in environmental variables and none of the variables was significant for the test of all effects (Table 4.5).

Table 4.5 Results of the top ten best fitting models of generalized linear model analyses (GLZ routine in STATISTICA v12) testing for the contribution of 6 environmental variables to explain variation in $F_{\mathrm{ST}}$ and $F_{\text {ST }}^{\prime}$ for Pecten novaezelandiae. The checkmarks $(\checkmark)$ indicate which variables were included in each model. ${ }^{1} \mathrm{p}$-value of each model; ${ }^{2} \mathrm{p}$-value of the test of all effects, significant values at $\mathrm{p}<0.05$ are in bold.

\begin{tabular}{|c|c|c|c|c|c|c|}
\hline \multicolumn{7}{|c|}{$F_{\mathrm{ST}}$ based on all 14 locations } \\
\hline $\mathrm{p}$-value ${ }^{1}$ & SST grad & Orb_v_mean & Tidal & FW & DOM & SPM \\
\hline$<0.001$ & $\checkmark$ & & $\checkmark$ & $\checkmark$ & $\checkmark$ & $\checkmark$ \\
\hline$<0.001$ & & & & $\checkmark$ & $\checkmark$ & $\checkmark$ \\
\hline$<0.001$ & & & $\checkmark$ & $\checkmark$ & $\checkmark$ & $\checkmark$ \\
\hline$<0.001$ & & $\checkmark$ & & $\checkmark$ & $\checkmark$ & $\checkmark$ \\
\hline$<0.001$ & $\checkmark$ & & & $\checkmark$ & $\checkmark$ & $\checkmark$ \\
\hline$<0.001$ & $\checkmark$ & $\checkmark$ & $\checkmark$ & $\checkmark$ & $\checkmark$ & $\checkmark$ \\
\hline$<0.001$ & & $\checkmark$ & $\checkmark$ & $\checkmark$ & $\checkmark$ & $\checkmark$ \\
\hline$<0.001$ & $\checkmark$ & $\checkmark$ & & $\checkmark$ & $\checkmark$ & $\checkmark$ \\
\hline$<0.001$ & $\checkmark$ & $\checkmark$ & $\checkmark$ & & $\checkmark$ & $\checkmark$ \\
\hline$<0.001$ & & $\checkmark$ & $\checkmark$ & & $\checkmark$ & $\checkmark$ \\
\hline $\mathrm{p}$-value ${ }^{2}$ & 0.060 & 0.604 & 0.039 & 0.024 & 0.005 & $<0.001$ \\
\hline \multicolumn{7}{|c|}{$F_{\text {ST }}^{\prime}$ based on all 14 locations } \\
\hline p-value ${ }^{1}$ & SST grad & Orb_v_mean & Tidal & FW & DOM & SPM \\
\hline$<0.001$ & & & & $\checkmark$ & $\checkmark$ & $\checkmark$ \\
\hline$<0.001$ & & $\checkmark$ & & $\checkmark$ & $\checkmark$ & $\checkmark$ \\
\hline$<0.001$ & $\checkmark$ & & & $\checkmark$ & $\checkmark$ & $\checkmark$ \\
\hline$<0.001$ & & $\checkmark$ & & & $\checkmark$ & $\checkmark$ \\
\hline$<0.001$ & & & $\checkmark$ & $\checkmark$ & $\checkmark$ & $\checkmark$ \\
\hline$<0.001$ & $\checkmark$ & & $\checkmark$ & $\checkmark$ & $\checkmark$ & $\checkmark$ \\
\hline$<0.001$ & $\checkmark$ & $\checkmark$ & & $\checkmark$ & $\checkmark$ & $\checkmark$ \\
\hline$<0.001$ & & $\checkmark$ & $\checkmark$ & $\checkmark$ & $\checkmark$ & $\checkmark$ \\
\hline$<0.001$ & & $\checkmark$ & $\checkmark$ & & $\checkmark$ & $\checkmark$ \\
\hline$<0.001$ & $\checkmark$ & $\checkmark$ & & & $\checkmark$ & $\checkmark$ \\
\hline $\mathrm{p}$-value ${ }^{2}$ & 0.253 & 0.578 & 0.366 & 0.148 & 0.076 & $<0.001$ \\
\hline
\end{tabular}


Table 4.5 (Cont.)

\begin{tabular}{|c|c|c|c|c|c|c|}
\hline \multicolumn{7}{|c|}{$F_{\mathrm{ST}}$ based on 11 locations (Excluding south) } \\
\hline p-value ${ }^{1}$ & SST grad & Orb_v_mean & Tidal & FW & DOM & SPM \\
\hline 0.470 & & & $\checkmark$ & & & \\
\hline 0.757 & $\checkmark$ & & & & & \\
\hline 0.758 & & & & $\checkmark$ & & \\
\hline 0.817 & & $\checkmark$ & & & & \\
\hline 0.860 & & & & & $\checkmark$ & \\
\hline 0.992 & & & & & & $\checkmark$ \\
\hline 0.488 & $\checkmark$ & & $\checkmark$ & & & \\
\hline 0.739 & & & $\checkmark$ & $\checkmark$ & & \\
\hline 0.769 & & & $\checkmark$ & & $\checkmark$ & \\
\hline 0.769 & & $\checkmark$ & $\checkmark$ & & & \\
\hline p-value ${ }^{2}$ & 0.291 & 0.872 & 0.214 & 0.993 & 0.668 & 0.676 \\
\hline \multicolumn{7}{|c|}{$F_{\text {ST }}^{\prime}$ based on 11 locations (Excluding south) } \\
\hline p-value ${ }^{1}$ & SST grad & Orb_v_mean & Tidal & FW & DOM & SPM \\
\hline 0.274 & & & & & $\checkmark$ & \\
\hline 0.354 & & & & & & $\checkmark$ \\
\hline 0.441 & & & $\checkmark$ & & & \\
\hline 0.567 & & & & $\checkmark$ & & \\
\hline 0.765 & $\checkmark$ & & & & & \\
\hline 0.982 & & $\checkmark$ & & & & \\
\hline 0.423 & $\checkmark$ & & $\checkmark$ & & & \\
\hline 0.483 & & & $\checkmark$ & & $\checkmark$ & \\
\hline 0.499 & & & $\checkmark$ & & & $\checkmark$ \\
\hline 0.507 & $\checkmark$ & & & & $\checkmark$ & \\
\hline $\mathrm{p}$-value ${ }^{2}$ & 0.206 & 0.953 & 0.185 & 0.746 & 0.831 & 0.554 \\
\hline
\end{tabular}

\section{BEST analyses}

BEST analyses based on data from all 14 locations and 9 geospatial and environmental variables showed that the top two best fitting models were significant at $\mathrm{p}<0.05$ and both included the variables Lat, Tidal, FW, DOM and SPM $\left(\mathrm{r}_{\mathrm{s}}=0.588\right)$. The remaining top eight best fitting models had similar Spearman's Rho value $(0.587$ and 0.586$)$ and the variables Lat and SPM were included in all models. The top ten best fitting models of BEST analyses based on data from 11 locations had low, non-significant Spearman's Rho values ranging from 0.340 to 0.335 , but the variables Lon and SPM were included in all models (Table 4.6). 
Table 4.6 Results of the ten best fitting models with respective Spearman's Rho $\left(\mathrm{r}_{\mathrm{s}}\right)$ values from the BEST analyses testing for the contribution of all 9 geospatial and environmental variables to explain variation in allele frequencies for Pecten novaezelandiae. The checkmarks $(\checkmark)$ indicate which variables were included in each model.

\begin{tabular}{|c|c|c|c|c|c|c|c|c|c|c|}
\hline \multicolumn{11}{|c|}{ All 14 locations } \\
\hline Rank & rs & Lat & Lon & Geo_dist & SST grad & Orb_v_mean & Tidal & FW & DOM & SPM \\
\hline 1 & 0.588 & $\checkmark$ & & & $\checkmark$ & & $\checkmark$ & $\checkmark$ & $\checkmark$ & $\checkmark$ \\
\hline 2 & 0.588 & $\checkmark$ & & & & & $\checkmark$ & $\checkmark$ & $\checkmark$ & $\checkmark$ \\
\hline 3 & 0.587 & $\checkmark$ & & & & & & $\checkmark$ & & $\checkmark$ \\
\hline 4 & 0.587 & $\checkmark$ & & & $\checkmark$ & & & $\checkmark$ & & $\checkmark$ \\
\hline 5 & 0.587 & $\checkmark$ & & & $\checkmark$ & & $\checkmark$ & $\checkmark$ & & $\checkmark$ \\
\hline 6 & 0.587 & $\checkmark$ & & & & & & $\checkmark$ & $\checkmark$ & $\checkmark$ \\
\hline 7 & 0.587 & $\checkmark$ & & & $\checkmark$ & & & $\checkmark$ & $\checkmark$ & $\checkmark$ \\
\hline 8 & 0.587 & $\checkmark$ & & & & & $\checkmark$ & $\checkmark$ & & $\checkmark$ \\
\hline 9 & 0.586 & $\checkmark$ & & & & & $\checkmark$ & & $\checkmark$ & $\checkmark$ \\
\hline 10 & 0.586 & $\checkmark$ & & & $\checkmark$ & & $\checkmark$ & & $\checkmark$ & $\checkmark$ \\
\hline
\end{tabular}

11 locations (Excluding south)

\begin{tabular}{|c|c|c|c|c|c|c|c|c|c|c|}
\hline Rank & $\mathrm{r}_{\mathrm{s}}$ & Lat & Lon & Geo_dist & SST grad & Orb_v_mean & Tidal & FW & DOM & SPM \\
\hline 1 & 0.340 & & $\checkmark$ & & & & & $\checkmark$ & & $\checkmark$ \\
\hline 2 & 0.340 & & $\checkmark$ & & $\checkmark$ & & & $\checkmark$ & & $\checkmark$ \\
\hline 3 & 0.338 & & $\checkmark$ & & & & $\checkmark$ & & $\checkmark$ & $\checkmark$ \\
\hline 4 & 0.338 & & $\checkmark$ & & $\checkmark$ & & $\checkmark$ & & $\checkmark$ & $\checkmark$ \\
\hline 5 & 0.337 & & $\checkmark$ & & & & & $\checkmark$ & $\checkmark$ & $\checkmark$ \\
\hline 6 & 0.337 & & $\checkmark$ & & $\checkmark$ & & & $\checkmark$ & $\checkmark$ & $\checkmark$ \\
\hline 7 & 0.336 & & $\checkmark$ & & $\checkmark$ & & $\checkmark$ & & & $\checkmark$ \\
\hline 8 & 0.336 & & $\checkmark$ & & & & & & & $\checkmark$ \\
\hline 9 & 0.336 & & $\checkmark$ & & $\checkmark$ & & & & & $\checkmark$ \\
\hline 10 & 0.335 & & $\checkmark$ & & & & $\checkmark$ & & & $\checkmark$ \\
\hline
\end{tabular}

When only the 3 geospatial variables were analysed, BEST analyses based on data from all 14 locations showed that the two best fitting models were significant at $\mathrm{p}<0.01$ with $\mathrm{r}_{\mathrm{s}}=0.560$ and included the variables Lat and Geo_dist. The remaining five best fitting models had very similar Spearman's Rho values ranging from 0.559 to 0.499 and included all the geospatial variables. BEST analyses based on data from 11 locations showed that the seven best fitting models were not significant and had low Spearman's Rho values ranging from 0.312 to 0.236 and all the variables were included in the models (Table 4.7). 
Table 4.7 Results of the ten best fitting models with respective Spearman's Rho $\left(\mathrm{r}_{\mathrm{s}}\right)$ values from the BEST analyses testing for the contribution of 3 geospatial variables to explain variation in allele frequencies for Pecten novaezelandiae. The checkmarks $(\checkmark)$ indicate which variables were included in each model.

\begin{tabular}{llccc}
\hline \multicolumn{5}{c}{ All 14 locations } \\
\hline Rank & $\mathrm{r}_{\mathrm{s}}$ & Lat & Lon & Geo_dist \\
\hline 1 & 0.560 & & & $\checkmark$ \\
2 & 0.560 & $\checkmark$ & & $\checkmark$ \\
3 & 0.559 & & $\checkmark$ & $\checkmark$ \\
4 & 0.559 & $\checkmark$ & $\checkmark$ & $\checkmark$ \\
5 & 0.558 & $\checkmark$ & & \\
6 & 0.501 & & $\checkmark$ & \\
7 & 0.499 & $\checkmark$ & $\checkmark$ & \\
\hline & 11 locations (Excluding south) & \\
\hline Rank & r & Lat & Lon & Geo_dist \\
\hline 1 & 0.312 & & $\checkmark$ & \\
2 & 0.306 & $\checkmark$ & $\checkmark$ & $\checkmark$ \\
3 & 0.289 & & & $\checkmark$ \\
4 & 0.289 & $\checkmark$ & & $\checkmark$ \\
5 & 0.285 & & $\checkmark$ & \\
6 & 0.285 & $\checkmark$ & $\checkmark$ & \\
7 & 0.236 & $\checkmark$ & &
\end{tabular}

When only the 6 environmental variables were analysed, BEST analyses based on data from all 14 locations showed that the ten best fitting models were not significant and had low Spearman's Rho values ranging from 0.327 to 0.234 . The variable SPM was included in all the models and the variable FW was included in the eight best fitting models. BEST analyses based on data from 11 locations also showed that the ten best fitting models were not significant and had low Spearman's Rho values ranging from 0.324 to 0.296 . The variables FW, DOM and SPM were included in the majority of the models (Table 4.8). 
Table 4.8 Results of the ten best fitting models with respective Spearman's Rho ( $\mathrm{r}_{\mathrm{s}}$ ) values from the BEST analyses testing for the contribution of 6 environmental variables to explain variation in allele frequencies for Pecten novaezelandiae. The checkmarks $(\checkmark)$ indicate which variables were included in each model.

\begin{tabular}{|c|c|c|c|c|c|c|c|}
\hline \multicolumn{8}{|c|}{ All 14 locations } \\
\hline Rank & $\mathrm{r}_{\mathrm{s}}$ & SST grad & Orb_v_mean & Tidal & FW & DOM & SPM \\
\hline 1 & 0.327 & $\checkmark$ & & & $\checkmark$ & & $\checkmark$ \\
\hline 2 & 0.326 & & & & $\checkmark$ & & $\checkmark$ \\
\hline 3 & 0.322 & & & & $\checkmark$ & $\checkmark$ & $\checkmark$ \\
\hline 4 & 0.322 & $\checkmark$ & & & $\checkmark$ & $\checkmark$ & $\checkmark$ \\
\hline 5 & 0.296 & $\checkmark$ & & $\checkmark$ & $\checkmark$ & & $\checkmark$ \\
\hline 6 & 0.295 & & & $\checkmark$ & $\checkmark$ & & $\checkmark$ \\
\hline 7 & 0.291 & & & $\checkmark$ & $\checkmark$ & $\checkmark$ & $\checkmark$ \\
\hline 8 & 0.291 & $\checkmark$ & & $\checkmark$ & $\checkmark$ & $\checkmark$ & $\checkmark$ \\
\hline 9 & 0.235 & & & & & & $\checkmark$ \\
\hline 10 & 0.234 & $\checkmark$ & & & & & $\checkmark$ \\
\hline
\end{tabular}

11 locations (Excluding south)

\begin{tabular}{|c|c|c|c|c|c|c|c|}
\hline Rank & $\mathrm{r}_{\mathrm{s}}$ & SST grad & Orb_v_mean & Tidal & FW & DOM & SPM \\
\hline 1 & 0.324 & & & & $\checkmark$ & $\checkmark$ & $\checkmark$ \\
\hline 2 & 0.322 & & & & $\checkmark$ & $\checkmark$ & \\
\hline 3 & 0.322 & $\checkmark$ & & & $\checkmark$ & $\checkmark$ & $\checkmark$ \\
\hline 4 & 0.320 & $\checkmark$ & & & $\checkmark$ & $\checkmark$ & \\
\hline 5 & 0.320 & & & & $\checkmark$ & & $\checkmark$ \\
\hline 6 & 0.320 & $\checkmark$ & & & $\checkmark$ & & $\checkmark$ \\
\hline 7 & 0.317 & $\checkmark$ & & & & $\checkmark$ & \\
\hline 8 & 0.299 & & & & & $\checkmark$ & $\checkmark$ \\
\hline 9 & 0.298 & & & & & $\checkmark$ & \\
\hline 10 & 0.296 & $\checkmark$ & & & & $\checkmark$ & $\checkmark$ \\
\hline
\end{tabular}

BEST analyses of locus-specific data showed that Spearman's Rho values ranged from 0.265 in Pnova_33 ( $>>0.05)$ to 0.572 in Pnova_04 $(\mathrm{p}<0.05)$ suggesting that genetic variation at some loci (as Pnova_04, Pnova_09 and Pnova_10) may be more affected by environmental variation than genetic variation at other loci. The variable Lat occurred 10 times in the top ten models for 7 loci, the variable Lon occurred 10 times for 2 loci and SPM occurred 10 times in the top ten models for 6 loci. Overall these results support the BEST analyses across all loci and suggest that individual locus effects are probably not occurring (Table 4.9). 
Table 4.9 Results of the BEST analyses testing for the contribution of 9 geospatial and environmental variables to explain variation in allele frequencies for each locus for Pecten novaezelandiae for all 14 locations. Top part of table represents the best fitting model for each locus; the checkmarks $(\checkmark)$ indicate which variables were included in the model. Bottom part of table indicates the number of times that each variable was included in the top ten best fitting models.

\begin{tabular}{|c|c|c|c|c|c|c|c|c|c|c|c|}
\hline Locus & $r_{s}$ & $\mathrm{p}$-value & Lat & Lon & Geo_dist & SST grad & Orb_v_mean & Tidal & FW & DOM & SPM \\
\hline Pnova_01 & 0.421 & 0.149 & & & & & & $\checkmark$ & $\checkmark$ & $\checkmark$ & $\checkmark$ \\
\hline Pnova_02 & 0.536 & 0.087 & $\checkmark$ & & & & & $\checkmark$ & & $\checkmark$ & $\checkmark$ \\
\hline Pnova_04 & 0.572 & 0.030 & $\checkmark$ & $\checkmark$ & & & & & $\checkmark$ & & $\checkmark$ \\
\hline Pnova_05 & 0.320 & 0.417 & & & & & & $\checkmark$ & $\checkmark$ & $\checkmark$ & \\
\hline Pnova_09 & 0.532 & 0.003 & $\checkmark$ & $\checkmark$ & & & & & $\checkmark$ & $\checkmark$ & $\checkmark$ \\
\hline Pnova_10 & 0.497 & 0.034 & $\checkmark$ & & & & & & & $\checkmark$ & \\
\hline Pnova_27 & 0.484 & 0.056 & $\checkmark$ & & & $\checkmark$ & & $\checkmark$ & $\checkmark$ & $\checkmark$ & $\checkmark$ \\
\hline Pnova_28 & 0.365 & 0.322 & $\checkmark$ & & & & & & & & \\
\hline Pnova_32 & 0.445 & 0.180 & $\checkmark$ & & & & & $\checkmark$ & & & $\checkmark$ \\
\hline Pnova_33 & 0.265 & 0.443 & & & & $\checkmark$ & & & $\checkmark$ & $\checkmark$ & \\
\hline Pnova_01 & - & - & 0 & 0 & 0 & 5 & 0 & 4 & 8 & 6 & 10 \\
\hline Pnova_02 & - & - & 10 & 0 & 0 & 4 & 0 & 7 & 3 & 5 & 10 \\
\hline Pnova_04 & - & - & 10 & 10 & $\mathbf{0}$ & 5 & $\mathbf{0}$ & 6 & 8 & 6 & 10 \\
\hline Pnova_05 & - & - & 0 & 0 & 0 & 4 & 0 & 8 & 6 & 5 & 0 \\
\hline Pnova_09 & - & - & 10 & 10 & $\mathbf{0}$ & 5 & $\mathbf{0}$ & 4 & 8 & 4 & 10 \\
\hline Pnova_10 & - & - & 10 & $\mathbf{0}$ & $\mathbf{0}$ & 5 & $\mathbf{0}$ & 2 & 6 & 6 & 0 \\
\hline Pnova_27 & - & - & 10 & 0 & 0 & 5 & 0 & 4 & 8 & 6 & 10 \\
\hline Pnova_28 & - & - & 10 & 0 & 0 & 5 & 0 & 4 & 2 & 6 & 0 \\
\hline Pnova_32 & - & - & 10 & 0 & 0 & 5 & 0 & 6 & 2 & 4 & 10 \\
\hline Pnova_33 & - & - & 1 & 4 & 4 & 4 & 4 & 3 & 6 & 4 & 0 \\
\hline
\end{tabular}


In summary, the main variables driving the genetic structure were longitude (Lon), freshwater fraction (FW) and suspended particulate matter (SPM). The variables latitude (Lat), index of geographic distance (Geo_dist), tidal current (Tidal), sea surface temperature (SSTgrad) and dissolved organic matter (DOM) also explained the genetic structure according to some of the models (Table 4.10).

Table 4.10 Summary of GLZ and BEST results with important variables explaining genetic variation as determined by the number of times they were included in the models, model pvalues, test of all effects $p$-values and significance of $r_{s}$ coefficients.

\begin{tabular}{ccc}
\hline Test & Analysis & Important variables \\
\hline$F_{\text {ST } 14 \text { locations }}$ & GLZ & Lon, Geo_dist, Tidal, FW, DOM, SPM \\
$F_{\text {ST } 14 \text { locations }}^{\prime}$ & GLZ & Lon, Tidal, FW, DOM, SPM \\
$F_{\text {ST } 11 \text { locations }}$ & GLZ & Lon, Tidal \\
$F_{\text {ST } 11 \text { locations }}^{\prime}$ & GLZ & All 9 variables \\
14 locations & BEST & Lat, FW, SPM \\
11 locations & BEST & Lon, FW, DOM, SPM \\
\hline
\end{tabular}

\section{Discussion}

In chapter 3, a low but significant level of population genetic structure of Pecten novaezelandiae was found between regions. While a degree of differentiation from north to south was apparent, it also conflicted with some evidence of panmixia. Seascape genetics can be a valuable tool for understanding these conflicting patterns of genetic differentiation and their association with the geographic and environmental variation (Riginos \& Liggins 2013). In this study, analyses showed that different combinations of environmental variables explain the genetic structure observed for $P$. novaezelandiae. In general, the main variables driving the genetic structure were longitude (Lon), freshwater fraction (FW) and suspended particulate matter (SPM). While the variables latitude (Lat), index of geographic 
distance (Geo_dist), tidal current (Tidal) and dissolved organic matter (DOM) explained the genetic structure according to some of the models, there was no evidence that spatial gradient annual mean sea surface temperature (SSTgrad) or mean orbital velocity (Orb_v_mean) were associated with the patterns of genetic differentiation observed.

Results showed that longitude explains genetic variation in terms of $F_{\mathrm{ST}}$ and $F_{\text {ST }}^{\prime}$ values for all 14 locations and allelic frequencies for 11 locations. Because sampling was based on the species distribution and was not continuous along the coast, samples were obtained from three groups of longitude values: a western group with values ranging from $+166^{\circ}$ (FIO) to $+168^{\circ}$ (STE14), a central group ranging from $+172^{\circ}$ (GOL) to $+176^{\circ}$ (TAU), and at the east the Chatham Islands at $-176^{\circ}$ (see Figure 4.1). The most differentiated populations were in the western group (FIO and STE14), so the results of seascape analyses might be biased by the distribution of the samples and reflect their differentiation. This might also be the case for the correlation of latitude with genetic variation, which was observed for allele frequencies when including all 14 locations. As the southern group (FIO and STE14) was the most differentiated, the results might reflect this hierarchical structure.

For most of the models, freshwater discharge was correlated with genetic variation in Pecten novaezelandiae. A significant association between genetic differentiation and salinity has been observed for the herring Clupea harengus (Jørgensen et al. 2005). Also, it has been observed a significant decrease in survivorship with the exposure to freshwater for the bivalve Paphies australis (McLeod \& Wing 2008) and more recently a correlation of genetic variation with the volume of freshwater entering the estuary (Hannan 2014). Biochemical genetic variation at the leucine aminopeptidase (LAP) locus has also been associated with salinity variation in Mytilus edulis in the U.S.A. (Hilbish 1985) and in Mytilus galloprovincialis in New Zealand (Gardner \& Palmer 1998). Although the present study does not test for causation, these results support the evidence that freshwater discharge can contribute to the population genetic variation of $P$. novaezelandiae.

There was strong evidence supporting the correlation of genetic variation $\left(F_{\mathrm{ST}}, F_{\mathrm{ST}}^{\prime}\right.$ and allelic frequencies) with levels of suspended particulate matter, except for $F_{\text {ST }}$ values when excluding the 3 locations in the south, which was probably due to lower levels of genetic variation among locations. Models that had significant correlation of genetic variation with levels of suspended particulate matter also showed some levels of correlation with 
dissolved organic matter, except for BEST analyses using 14 locations. Given that coastal areas are generally influenced by similar sources of suspended particulate matter and dissolved organic matter (D'Sa et al. 2007) it is not unreasonable to expect similar results.

It is known that bivalves' response to different concentrations of suspended particulate matter differs substantially among species. Bricelj \& Malouf (1984) suggested that bivalves such as clams and scallops that regulate ingestion primarily by reducing clearance rates are more susceptible to high concentrations of suspended sediment than mussels and oysters, which control ingestion mainly by increasing pseudofaecal production. However, studies suggest that intermediate strategies may occur and the ability to cope with different levels of suspended particulate matter may also vary within a species (Navarro \& Iglesias 1993). It has been shown that prolonged exposure to suspended particulate matter can affect both the feeding behaviour and growth rate of the scallop Pecten maximus. Although elevated suspended particulate matter did not have any short-term effects on survival of the scallops, significantly lower growth rates were observed under both low and high levels of suspended particulate matter (Szostek et al. 2013). Higher concentrations of suspended particulate matter can affect, for example, feeding and respiration of Pecten novaezelandiae (by gill-clogging) and might further modify their genetic variation by favouring locally adapted individuals. Therefore, different levels of suspended particulate matter among locations can be one of the factors contributing to population genetic structure of $P$. novaezelandiae.

According to some of the GLZ models, genetic variation was correlated with the index of geographic distance between locations. These results are in agreement with the significant isolation by distance signal detected in chapter 3 suggesting that the distance between locations is an important explanatory variable. The pattern of isolation by distance, where all populations are connected by continuous migration but gene flow is higher between nearby populations, has been shown for other bivalves such as the sea scallop Placopecten magellanicus (Kenchington et al. 2006) and the softshell clam Mya arenaria (St-Onge et al. 2013). However, for Pecten novaezelandiae it appears that the level of genetic differentiation is not a simple function of the geographic distance between populations. For example, it is not evident that the distance between the Chatham Islands and the mainland acts as an important barrier to larval dispersal. Other environmental or geospatial factors beyond the ones tested in this study could be playing an important role in 
explaining genetic variation. For example, the subtropical convergence flowing eastward might be facilitating gene flow and thus minimising the impact of geographic distance between the Chatham Islands and the mainland New Zealand.

Tidal current was one of the environmental factors explaining the genetic structure according to some of the models. In the Coromandel fishery for example, scallops are more common in depths of 10 to $30 \mathrm{~m}$ (Ministry of Primary Industries 2013a), so the flow of tidal currents could still represent a selective agent for $P$. novaezelandiae. The littorine snail Littorina saxatilis shows strong evidence for selection at a single locus (allozyme locus Aat; EC. 2.6.1.1). The enzyme aspartate aminotransferase plays an important role in amino acid metabolism and while $A a t^{120}$ is most frequent in high shore areas of Northern Europe, $\mathrm{Aat}^{100}$ is more common in low shore areas (Johannesson \& Johannesson 1989; Johannesson et al. 1995). There is also considerable evidence for adaptive variation associated with distinct intertidal microhabitats at the glucose phosphate isomerase locus (Gpi) and the mannose phosphate isomerase (Mpi) locus in the northern acorn barnacle Semibalanus balanoides (Rand et al. 2002).

Sea surface temperature has been identified as one important explanatory factor for the observed genetic variation in other species (Wei et al. 2013b; Constable 2014; Hannan 2014) and it is one important environmental factor triggering spawning in scallops (Shumway \& Parsons 2006). In this study, a few models related SSTgrad with genetic variation, and for locus-specific data this variable was included 4 to 5 times in the top ten best-fitting models. Although there was not strong support for a correlation between temperature and genetic variation of $P$. novaezelandiae, these results may be masked by the significant importance of other environmental factors.

In general, for the BEST analyses, correlations with the geospatial variables for both scenarios (for all locations and excluding south) were higher compared to correlations with environmental variables only. This suggests that geospatial variables might be an important factor determining the large-scale population genetic structure of Pecten novaezelandiae. However, as suggested by GLZ and BEST analyses, other environmental factors such as levels of suspended particulate matter and freshwater input could also be affecting larval settlement, feeding behaviour and growth rate of the New Zealand scallop, and therefore contributing to an increase in genetic differentiation among populations. 
Other unknown factors could also be limiting larval dispersal between populations but are undetected in this study. For example, Talman et al. (2004) showed that survival of juvenile Pecten novaezelandiae was affected by the ambient scallop density and that adult scallop predation increased with a smaller number of other species such as sponges and ascidians. In addition, ocean currents and hydrodynamic forces such as eddies and current discontinuities can strongly affect gene flow and population structure (White et al. 2010). This study provides valuable information not only for a better understanding of population genetics of $P$. novaezelandiae but also for identifying common environmental factors affecting population genetic structure of multiple species. Although the mechanisms behind local adaptation are unknown, studies like this one provide important insights on the processes shaping marine population structure and form a basis for identifying suitable management strategies across species.

\section{Conclusions}

In general, results of this study suggest that a combination of variables might be influencing the population genetic structure of Pecten novaezelandiae. Although several population genetic studies highlight the importance of geographic distance between populations, for Pecten novaezelandiae it appears that the level of genetic differentiation is not a simple function of this geospatial variable. There were strong evidences that levels of suspended particulate matter can be one of the factors contributing to population genetic structure of $P$. novaezelandiae.

Coastal marine environments often experience a large variation in levels of suspended particulate matter that can be driven by oceanographic cycles, wave action and high rainfall resulting in increased river output (Hall 1994). The increase of input of sediments into coastal areas can also be associated with the development of coastal regions and land use such as farming or forestry. This increase in suspended sediment can greatly alter the structure and function of coastal ecosystems including decreasing photosynthetic output and smothering of plants and animals (Thrush et al. 2004). As both ecosystems are highly linked, it is very important to account for these effects on marine ecosystems when planning land management and conservation practices (Stoms et al. 2005). 
As for most seascape genetic studies to date, the current study is not able to test for causation. Other techniques such as direct observation of dispersal and reproduction or functional genomics would be required to evaluate the link between genetic variation and a particular spatial or environmental factor (Liggins et al. 2013). Another limitation of this study is that extreme events and temporal variation of the environmental data are not specifically represented in the averaged mean and these environmental oscillations can highly influence gene flow and the genetic structure of organisms. While more environmental and genetic data have become available for seascape genetic studies, studies are still scarce and patchy. Therefore, interpretations of results are still limited. Nevertheless, the application of seascape genetics is a valuable tool, particularly for marine species exhibiting a weak genetic signal, which is the case of Pecten novaezelandiae. By identifying previously unknown environmental factors that can be driving genetic variation, the emerging field of seascape genetics provides essential clues for further research guidelines. 
CHAPTER 5

Spatial and temporal patterns of dispersal of Pecten

novaezelandiae in the Coromandel fishery: implications for management 


\section{Introduction}

Abundance and distribution of highly fecund marine species with a pelagic larval stage can vary greatly in space and time. The causes of variation in larval dispersal are difficult to assess and contrasting results are commonly reported across space, time and taxa (Levin 2006; Gardner et al. 2010). The use of different tools that provide independent estimates of population genetic structure is a powerful approach to understand larval dispersal and marine connectivity. Using only one sampling scale in population genetic studies may not detect some important factors influencing gene flow (Anderson et al. 2010). Although employing different strategies cannot exclude all sources of noise, they increase precision of estimating the factors that shape population genetic structure.

Complex genetic patterns can arise even in marine species that do not have obvious barriers to gene flow. As a result, some broadcast spawning marine invertebrates can exhibit small-scale spatial genetic structure (e.g. Wood \& Gardner 2007; Zhan et al. 2009; Owen \& Rawson 2013). Restricted dispersal of larvae can be the result of (1) high levels of self-recruitment, (2) high mortality rates, and/or (3) fine scales of larval transport (reviewed in Pineda et al. 2007). However, the distance that larvae can travel, their place and timing of final settlement, and their subsequent successful reproduction depend on many interdependent factors such as biological features (e.g. larval behaviour), local hydrodynamic characteristics such as eddies and gyres, and the habitat availability at settlement locations (reviewed in Swearer et al. 2002).

Temporally variable genetic structure is sometimes observed among some marine invertebrates (e.g. Owen \& Rawson 2013; Yednock \& Neigel 2014) and can potentially influence interpretations about spatial patterns of differentiation (Toonen \& Grosberg 2011). Unexpected temporal patterns can be determined by a large variance in reproductive success rates. The hypothesis of Sweepstakes Reproductive Success (SRS), postulates that very fecund marine animals with high early mortality rates have high variance in individual reproductive success and therefore only a small number of adults contribute to the next generation (Hedgecock 1982). As a result, juveniles are expected to exhibit reduced genetic diversity, higher relatedness and smaller effective population sizes. In addition, SRS can greatly contribute to the patterns of genetic differentiation and can also result in chaotic genetic patchiness (Johnson \& Black 1982; Pujolar et al. 2006; Hogan et al. 2010; 
Hedgecock \& Pudovkin 2011; Broquet et al. 2013). A lack of temporal stability can also be the result of stochastic factors, such as variation in oceanic currents, which can affect the movement of larvae and consequently the genetic patterns (Sotka et al. 2004; Selkoe \& Toonen 2006; Pringle \& Wares 2007). Nonetheless, in most studies, only spatial patterns of genetic structure are examined and the possibility for temporal genetic variation in scallops has been rarely addressed (Lewis \& Thorpe 1994; Owen \& Rawson 2013).

One of the main goals for fisheries management is to avoid depletion of fish stocks and a crucial prerequisite is to match biologically relevant processes with management action (Reiss et al. 2009). Therefore, understanding population dynamics and patterns of connectivity is vital for the delimitation of spatial management areas with meaningful biological units. Marine species may reside in "source" or "sink" habitats, where populations occurring in "sink" habitats only make a small contribution of juveniles to the spawning stock and populations in "source" habitats contribute an excess of individuals that sustains populations both in source and sink habitats (Pulliam 1988). These source-sink dynamics have been observed for several marine species (e.g. Lipcius et al. 1997; Chiswell \& Booth 2008; Barson et al. 2009; Drake \& Griffen 2013; Thomas \& Bell 2013) and it has important implications for fisheries management. Exploiting a source population can markedly reduce productivity of a sink population and for example, marine protected areas could provide a continuous source of recruitment and bring important environmental and economic benefits (Tuck \& Possingham 2000).

It has been demonstrated for some scallop species that populations separated by only a few tens to hundreds of kilometres show genetic differentiation. For example, individuals of the Zhikong Scallop (Chlamys farreri) collected from different marine gyres showed significant genetic differentiation (Zhan et al. 2009) and the genetic structure of the Atlantic sea scallop (Placopecten magellanicus) coincides with the ocean current structure of the Eastern Maine Coastal Current (Owen \& Rawson 2013). This information on population dynamics of species at fine spatial scales is crucial when delineating meaningful management units because separate stocks may correspond to geographic areas that are smaller than expected for marine species.

The New Zealand endemic scallop Pecten novaezelandiae supports very important fisheries, particularly in the Coromandel region. The Coromandel scallop fishery encompasses the area between Cape Rodney in the north and Town Point in the south 
(Ministry of Primary Industries 2013a). Scallops occur in dense aggregations or "beds" in the region and although they are not completely sedentary, Morrison (1999) found that adults rarely move more than $10 \mathrm{~m}$. Although scallops are managed as one single stock in the Coromandel (Ministry of Primary Industries 2013a), there is no previous information on the genetic connectivity between these "beds". The Coromandel region is a key marine environment in New Zealand, supporting large wild fisheries and at the same time being on the edge of New Zealand's largest city (Auckland, 1.2 million population). The Coromandel fishery area encompasses the Hauraki Gulf, a semi-enclosed area of northeastern New Zealand, and an open coastal environment both characterized by complex hydrodynamics. The East Auckland Current (EAUC) flowing southeast influences the physical oceanography (Zeldis et al. 2004) and nutrient supply (Zeldis 2004) of both environments, the Gulf and the open coast. Depending on the predominant currents and gyre dynamics, gene flow between scallop aggregations or self-recruitment within aggregations may be facilitated. Therefore, understanding patterns of dispersal of scallops in the region is essential for an effective management and provides important knowledge about the processes that influence source-sink dynamics of populations.

In chapter 3, the first genetic structure study of Pecten novaezelandiae was conducted, encompassing the full distributional range of the species. The present chapter aimed to estimate the spatial and temporal patterns of genetic differentiation of Pecten novaezelandiae in the Coromandel fishery region using 10 microsatellite markers and a hydrodynamic modelling approach to track the dispersal path of larvae released at three locations. Temporal analysis combined with a geographic analysis of genetic structure allows inferences on whether genetic variation among populations is due to self-recruitment or sporadic recruitment from another genetically distinct source population. This study also aimed to identify source-sink relationships. If a model of source-sink dynamics occurs, an asymmetrical dispersal between two populations is expected, with individuals predominantly dispersing from the source to the sink population. However, the processes that result in source-sink dynamics are not well understood. Therefore, this study aimed to provide insight into the processes that shape recruitment of populations, with particular applications to the Coromandel scallop fishery. 


\section{Materials and methods}

\section{Sampling}

A total of 592 individual scallops, Pecten novaezelandiae, were collected from the Coromandel region of northern New Zealand for this study. Data were analysed in two groups: a spatial analysis using 5 locations sampled in 2012 (Figure 5.1a, Table 5.1a) and a temporal analysis from 3 locations using a reduced data set of samples from 2012 (chapter 3) and additional samples from the same locations collected in 2014 (Figure 5.1b, Table 5.1b). Therefore, a subset of larger scallops collected in 2012 and smaller scallops collected in 2014 were used to discern individuals derived from different recruitment seasons to test for the temporal stability of patterns of genetic structure.

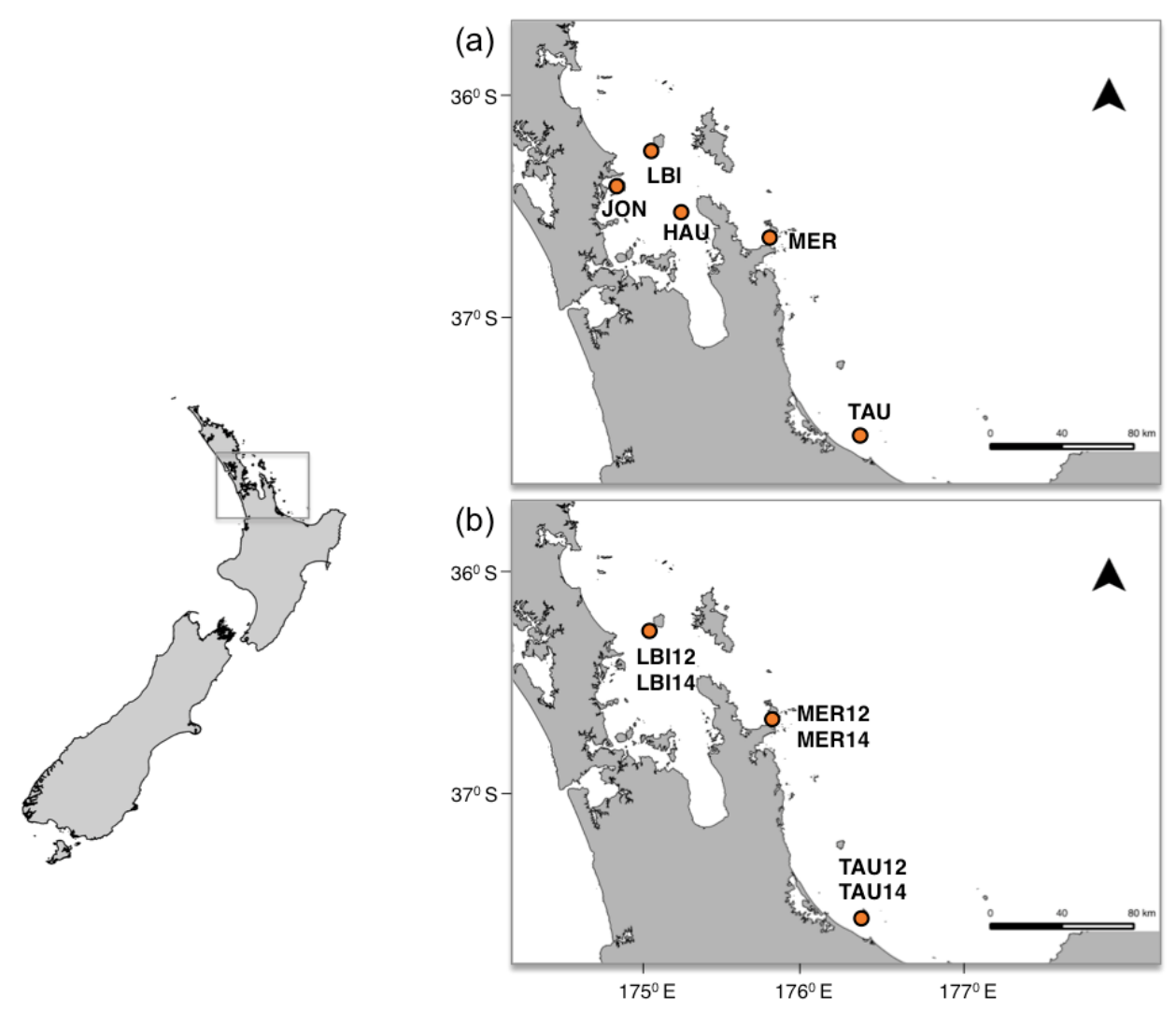

Figure 5.1 Map of sampling sites in the Coromandel region: (a) for the spatial analyses individuals of Pecten novaezelandiae were collected in 2012 and (b) for the temporal analyses a subset of individuals collected in 2012 and additional samples collected in 2014 was used. Locations abbreviations as per Table 5.1. 
All the individuals were measured (left shell length) and size structure was analysed using the software R 3.1.0 (R Core Team 2014). Individuals were genotyped for 10 loci (detailed laboratory protocols are described in Chapters 2 and 3) and the additional 192 samples collected in 2014 were analysed as per Chapters 2 and 3.

Table 5.1 Collecting site descriptions for the (a) spatial and (b) temporal analyses of Pecten novaezelandiae in the Coromandel region with sample sizes $(\mathrm{N})$, mean depth $(\mathrm{m})$, dates and geospatial coordinates.

(a)

\begin{tabular}{lllllll}
\hline Code & $\begin{array}{l}\text { Site } \\
\text { Description }\end{array}$ & $\mathrm{N}$ & $\begin{array}{l}\text { Depth } \\
(\mathrm{m})\end{array}$ & $\begin{array}{l}\text { Date } \\
\text { collected }\end{array}$ & Latitude & Longitude \\
\hline LBI & Little Barrier Is & 95 & 15 & $1 / 05 / 2012$ & $-36^{\circ} 13^{\prime} 51.0^{\prime \prime}$ & $+175^{\circ} 4^{\prime} 17.40^{\prime \prime}$ \\
JON & Jones Bay & 40 & 10 & $15 / 11 / 2012$ & $-36^{\circ} 22^{\prime} 53.6^{\prime \prime}$ & $+174^{\circ} 48^{\prime} 59.48^{\prime \prime}$ \\
HAU & Hauraki Gulf & 99 & 43 & $28 / 11 / 2012$ & $-36^{\circ} 34^{\prime} 9.60^{\prime \prime}$ & $+175^{\circ} 14^{\prime} 6.57^{\prime \prime}$ \\
MER & Mercury Is & 69 & 15 & $1 / 05 / 2012$ & $-36^{\circ} 41^{\prime} 3.60^{\prime \prime}$ & $+175^{\circ} 43^{\prime} 33.60^{\prime \prime}$ \\
TAU & Tauranga & 99 & 22 & $3 / 05 / 2012$ & $-37^{\circ} 40^{\prime} 14.4^{\prime \prime}$ & $+176^{\circ} 24^{\prime} 10.80^{\prime \prime}$ \\
\hline Total & & 402 & & & & \\
\hline
\end{tabular}

(b)

\begin{tabular}{lllllll}
\hline Code & $\begin{array}{l}\text { Site } \\
\text { Description }\end{array}$ & $\mathrm{N}$ & $\begin{array}{l}\text { Depth } \\
(\mathrm{m})\end{array}$ & $\begin{array}{l}\text { Date } \\
\text { collected }\end{array}$ & Latitude & Longitude \\
\hline LBI12 & Little Barrier Is & 63 & 15 & $1 / 05 / 2012$ & $-36^{\circ} 13^{\prime} 51.0^{\prime \prime}$ & $+175^{\circ} 44^{\prime} 17.40^{\prime \prime}$ \\
LBI14 & Little Barrier Is & 64 & 16 & $6 / 07 / 2014$ & $-36^{\circ} 12^{\prime} 40.0^{\prime \prime}$ & $+175^{\circ} 2^{\prime} 32.00^{\prime \prime}$ \\
MER12 & Mercury Is & 60 & 15 & $1 / 05 / 2012$ & $-36^{\circ} 41^{\prime} 3.60^{\prime \prime}$ & $+175^{\circ} 43^{\prime} 33.60^{\prime \prime}$ \\
MER14 & Mercury Is & 60 & 23 & $6 / 07 / 2014$ & $-36^{\circ} 40^{\prime} 25.0^{\prime \prime}$ & $+175^{\circ} 42^{\prime} 22.00^{\prime \prime}$ \\
TAU12 & Tauranga & 70 & 22 & $3 / 05 / 2012$ & $-37^{\circ} 40^{\prime} 14.4^{\prime \prime}$ & $+176^{\circ} 24^{\prime} 10.80^{\prime \prime}$ \\
TAU14 & Tauranga & 66 & 25 & $6 / 07 / 2014$ & $-37^{\circ} 37^{\prime} 57.0^{\prime \prime}$ & $+176^{\circ} 20^{\prime} 14.00^{\prime \prime}$ \\
\hline Total & & 383 & & & & \\
\hline
\end{tabular}

\section{Analysis of genetic diversity}

Genotyping artefacts were assessed using the software Micro-Checker v.2.2.0.3 (Van Oosterhout et al. 2004). Analyses of departure from Hardy-Weinberg equilibrium (HWE) and linkage disequilibrium (LD) were performed using the software GenePop on the web using the Markov chain method and Fisher's exact test (Rousset 2008). False discovery rate (FDR) control (Verhoeven et al. 2005) was applied to p-values in all statistical analyses that included multiple comparisons. 
The software Lositan (Antao et al. 2008) was used to perform an outlier analysis with 50,000 simulations and a confidence interval (CI) of 0.95 under the infinite alleles model (IAM) for a sample size of 50 .

The software HP-Rare (Kalinowski 2005) was used to quantify genetic diversity as allelic richness $\left(\mathrm{A}_{\mathrm{R}}\right)$ and private allelic richness $\left(\mathrm{P}_{\mathrm{AR}}\right)$ with a rarefaction sample size of 60 genes for the spatial analyses and 112 genes for the temporal analyses. GenAlEx 6.5 (Peakall \& Smouse 2012) was used to quantify the number of private alleles per site $(\mathrm{Pa})$, observed $\left(\mathrm{H}_{\mathrm{O}}\right)$ and expected $\left(\mathrm{H}_{\mathrm{E}}\right)$ heterozygosity and the fixation index $\left(F_{\mathrm{IS}}\right)$.

\section{Population structure}

The software GenePop on the web (Rousset 2008) was used to calculate pairwise $F_{\mathrm{ST}}$ values (adapted by Weir \& Cockerham, 1984). An exact G-test (Goudet et al. 1996) was also calculated using the $\mathrm{G} \log$ likelihood ratio with 10,000 dememorization steps, 1,000 batches and 10,000 iterations per batch for each pair. All p-values were corrected using the False Discovery Rate (FDR) (Verhoeven et al. 2005) at $\mathrm{p}<0.05$.

The neighbour joining (NJ) method (Saitou \& Nei 1987) was used to generate a population tree using the software PopTreeW (Takezaki et al. 2014) based on $F_{\mathrm{ST}}$ values with 1000 bootstrap replicates. A principal components analysis (PCoA) was performed in GenAlEx 6.5 (Peakall \& Smouse 2006) to test for variation among locations using codominant genetic distance.

A non-parametric analysis was conducted in the software package AWclust (Gao \& Starmer 2008) to evaluate population genetic structure among locations, following the approach of Gruber et al. (2013) and Wei et al. (2013a). As a comparison, a Bayesian cluster analysis was also performed in Structure 2.3.4 (Pritchard et al. 2000) using 100,000 interactions, a burn-in length of 10,000, 'admixture' model, 'correlated allele frequencies' and sampling regions were used as 'prior' for $\mathrm{k}=1$ through $\mathrm{k}=5$ for the spatial analyses and through $\mathrm{k}=6$ for the temporal analyses. Results were evaluated using the software Structure Harvester (Earl \& VonHoldt 2012) and the appropriate number of clusters was determined by the $\log$ probability $(\mathrm{L}(\mathrm{K}))$ and $\Delta \mathrm{K}$ across multiple runs according to Evanno et al. (2005).

An Analysis of Molecular Variation (AMOVA) was performed in GenAlEx 6.5 (Peakall $\&$ Smouse 2012) using 999 permutations. For the spatial analyses, the 5 locations were 
grouped into 3 regions (1: LBI, HAU, 2: JON, 3: MER, TAU) and 4 regions (1: LBI, 2: HAU, 3: JON, 4: MER, TAU) to test for the significance of the PCoA and the neighbour joining analysis. For the temporal analyses, the 6 locations were grouped in 2 regions for a temporal test between years using all locations (1: LBI12, MER12, TAU12, 2: LBI14, MER14, TAU14) and for a temporal test between years for locations MER and TAU (1: MER12, TAU12, 2: MER14, TAU14).

\section{Estimates of migration}

The Bayesian program Geneclass 2 (Piry et al. 2004) was used to identify firstgeneration migrants as well as the proportion of individuals recruiting to a location, using the Markov chain Monte Carlo (MCMC) and the algorithm of Paetkau et al. (2004). The likelihood ratio 'L_home/L_max' was used with a 0.01 rejection level and a simulated population size of 10,000 individuals per site. This approach selects an individual's genotype from the location in which it was sampled over the highest likelihood observed for this genotype in any location (Paetkau et al. 2004). The individuals were assigned to the location with the highest probability.

\section{Relatedness}

To test whether scallop samples from 2014 had a greater number of relatives than the samples from 2012, the mean population relatedness (r) (Queller and Goodnight 1989) was calculated in GenAlEx 6.5 (Peakall \& Smouse 2006) across all locations using 999 bootstrap permutations for significance. The measure $r$ is calculated based on the genetic similarity between two individuals in relation to the similarity between random individuals in a reference population (Pamilo 1990).

\section{Oceanographic model}

The online marine connectivity interface ConnIe2 (CSIRO 2014) was used to investigate the advection of propagules by oceanic currents in the Coromandel region. ConnIe 2 was developed using OFAM (Ocean Forecasting Australia Model) which, in turn, was developed using the Modular Ocean Model (MOM4) code as part of the BLUElink Project. This three-dimensional nonlinear hydrodynamic model has a spatial resolution of 0.1 degrees $(\sim 10 \mathrm{~km})$, so a finer spatial resolution would be necessary for simulations in the 
inner part of the Hauraki Gulf. Consequently, connectivity was estimated for the open coastal environment using the embedded particle-tracking module under three different scenarios: particles released from Little Barrier Island (LBI), from Mercury Island (MER) and from Tauranga (TAU). The behavioural trait of diel vertical migration was incorporated (Kaartvedt et al. 1987), particles were released in early summer (the main dispersal period of $P$. novaezelandiae in the Coromandel region) and tracked for a larval duration of 25 days (the approximate pelagic larval duration), and results were averaged across ten years (1997-2007), the latest data available.

\section{Results}

Of the 592 scallops analysed, 402 were employed in the spatial analyses and 383 were employed in the temporal analyses. For the spatial analyses all individuals were $>65 \mathrm{~mm}$ in shell length and classified as mature (Williams \& Babcock 2005). The average size of all individuals was $99.31 \pm 6.70 \mathrm{~mm}$ (Figure 5.2a) and at this length they are likely to have recruited 1.5 years prior to collection (i.e., 2010-2011). For the temporal analyses, all individuals were $>70 \mathrm{~mm}$ in shell length and classified as mature (Williams \& Babcock 2005) with an average size of $96.57 \pm 7.67 \mathrm{~mm}$. The average size of individuals collected in 2012 was $101.36 \pm 5.53 \mathrm{~mm}$. Individuals collected in 2014 had an average size of 91.42 $\pm 6.18 \mathrm{~mm}$ and at this length they are likely to have recruited in 2012-2013 (Figures 5.2b and 5.3).

(a)

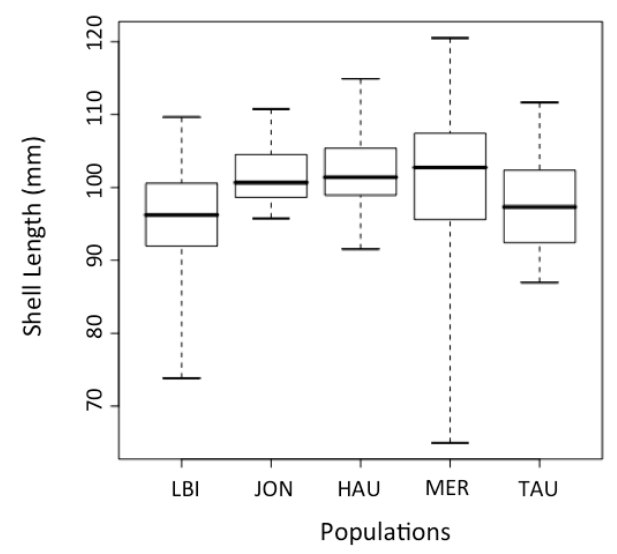

(b)

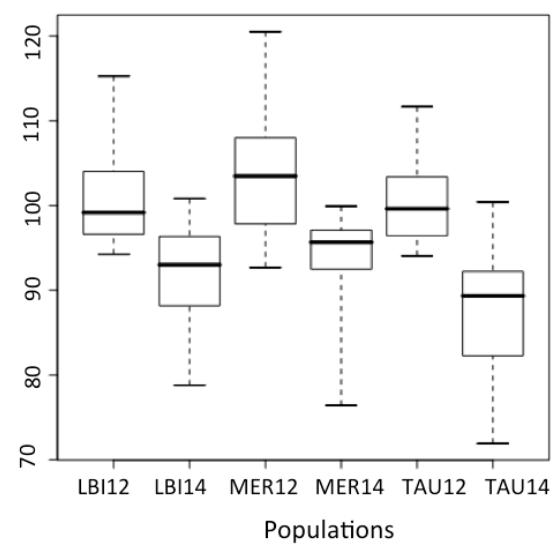

Figure 5.2 Size structure of individuals of Pecten novaezelandiae collected in the Coromandel region for the (a) spatial and (b) temporal studies. Dashed lines represent the maximum and minimum size at each site, while the bars represent the 25th and 75th 
quartile of size ranges, with the median indicated within each bar. Location abbreviations as per Table 5.1
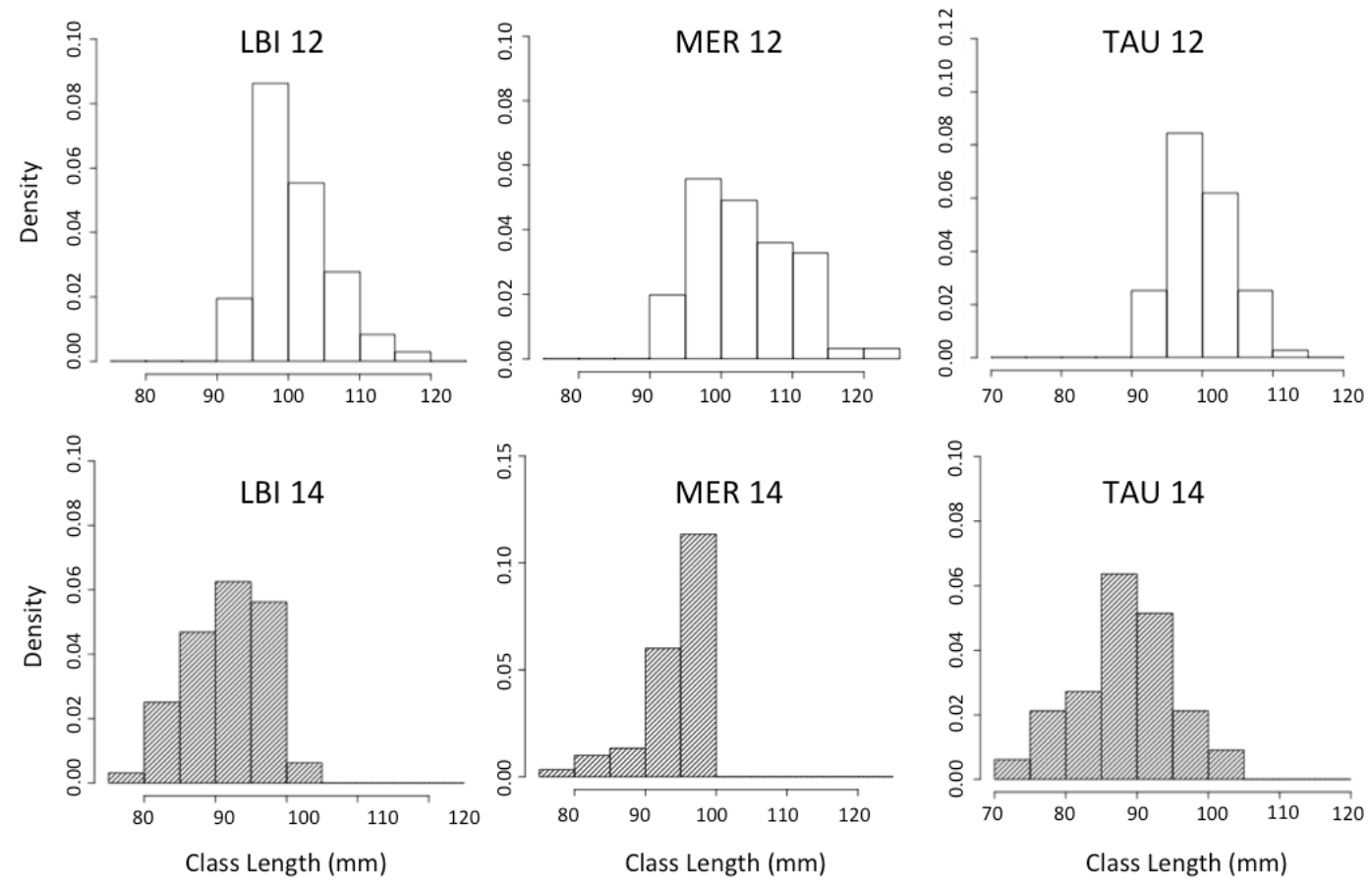

Figure 5.3 Length-frequency histograms of Pecten novaezelandiae collected in 2012 and 2014 in the Coromandel region for the temporal analyses. Location abbreviations as per Table 5.1.

\section{Genetic diversity}

For the spatial analyses, Micro-Checker analyses identified putative null alleles at Pnova_01, Pnova_02, Pnova_05, Pnova_10,Pnova_27 and Pnova_33 due to homozygote excess and no long allele dropout was detected. After FDR correction for multiple tests, Pnova_10 was identified as being significantly out of HWE at all locations and Pnova_27 at more than half of the locations. There was no evidence of significant linkage disequilibrium between locus pairs. Lositan analyses identified all 10 loci as neutral markers. Allelic richness ranged from 9.78 at TAU to 10.07 at MER. Private allelic richness ranged from 0.48 at TAU to 0.65 at HAU. In total there were 33 private alleles across all locations; LBI had the greatest number (9). The observed heterozygosity ranged from 0.564 at HAU to 0.662 at MER, and expected heterozygosity ranged from 0.658 at TAU to 0.671 at HAU. Location MER had the lowest value of $F_{\text {IS }}(0.006)$ and HAU had 
the highest value (0.146). All locations showed significant departures from HWE expectations after FDR correction, except MER (Table 5.2a).

For the temporal analyses, putative null alleles were identified at Pnova_01, Pnova_02, Pnova_10,Pnova_27, Pnova_28 and Pnova_33. After FDR correction for multiple tests, Pnova_10 and Pnova_27 were identified as being significantly out of HWE at more than half of the locations. There was no evidence of significant linkage disequilibrium between locus pairs. All 10 loci were identified as neutral markers. Allelic richness ranged from 10.73 at MER14 to 11.54 at MER12. Private allelic richness ranged from 0.11 at TAU14 to 0.65 at MER12. In total there were 25 private alleles across all locations; MER12 and TAU12 had the highest number (7 each). Overall, there were lower levels of allelic richness and private allelic richness as well as higher values of $F_{\text {IS }}$ in samples collected in 2014 than those collected in 2012. All locations showed significant departure from HWE expectations after FDR correction, except MER12 (Table 5.2b).

Table 5.2 Genetic variation in P. novaezelandiae in the Coromandel region for locations used for (a) the spatial study and (b) the temporal study. Allelic richness $\left(A_{R}\right)$, private allelic richness $\left(\mathrm{PA}_{\mathrm{R}}\right)$, number of private alleles per site $(\mathrm{Pa})$, observed heterozygosity $\left(\mathrm{H}_{\mathrm{O}}\right)$, expected heterozygosity $\left(\mathrm{H}_{\mathrm{E}}\right)$ and fixation index $\left(\mathrm{F}_{\mathrm{IS}}\right)$. $\mathrm{F}_{\mathrm{IS}}$ values in bold show significant departure from HWE expectations after FDR correction $(\mathrm{p}<0.05)$. Locations abbreviations as per Table 5.1.

(a)

\begin{tabular}{ccccccc}
\hline Location & $\mathbf{A}_{\mathbf{R}}$ & $\mathbf{P A}_{\mathbf{R}}$ & $\mathbf{P a}$ & $\mathbf{H}_{\mathbf{O}}$ & $\mathbf{H}_{\mathbf{E}}$ & $\mathbf{F}_{\mathbf{I S}}$ \\
\hline LBI & 10.00 & 0.55 & 9 & 0.605 & 0.665 & $\mathbf{0 . 0 8 8}$ \\
JON & 9.79 & 0.51 & 5 & 0.596 & 0.668 & $\mathbf{0 . 1 1 5}$ \\
HAU & 9.94 & 0.65 & 6 & 0.564 & 0.671 & $\mathbf{0 . 1 4 6}$ \\
MER & 10.07 & 0.56 & 6 & 0.662 & 0.670 & 0.006 \\
TAU & 9.78 & 0.48 & 7 & 0.596 & 0.658 & $\mathbf{0 . 0 8 3}$
\end{tabular}

(b)

\begin{tabular}{lllllll} 
LBI12 & 11.39 & 0.33 & 5 & 0.608 & 0.660 & $\mathbf{0 . 0 7 1}$ \\
LBI14 & 10.93 & 0.32 & 4 & 0.579 & 0.660 & $\mathbf{0 . 1 2 9}$ \\
MER12 & 11.54 & 0.65 & 7 & 0.666 & 0.670 & 0.002 \\
MER14 & 10.73 & 0.12 & 1 & 0.556 & 0.646 & $\mathbf{0 . 1 3 9}$ \\
TAU12 & 11.29 & 0.45 & 7 & 0.593 & 0.651 & $\mathbf{0 . 0 6 8}$ \\
TAU14 & 10.74 & 0.11 & 1 & 0.599 & 0.671 & $\mathbf{0 . 0 9 6}$ \\
\hline
\end{tabular}




\section{Population structure}

For the spatial study, $F_{\text {ST }}$ values for all comparisons ranged from 0.0004 to 0.0033 . Of the 10 comparisons, 3 were significant after FDR correction for multiple comparisons (Table 5.3). For the temporal study, $\mathrm{F}_{\mathrm{ST}}$ values for all comparisons ranged from 0 to 0.0048 . Of the 15 comparisons, 5 were significant after FDR correction for multiple comparisons. Interestingly, locations MER12-MER14 and TAU12-TAU14 were significantly different but not the locations LBI12-LBI14 (Table 5.4).

Table 5.3 Pairwise FST values for Pecten novaezelandiae for the spatial study are below the diagonal and p-values are above the diagonal. Significant values are in bold after FDR testing $(\mathrm{p}<0.05)$. Locations abbreviations as per Table 5.1.

\begin{tabular}{cccccc}
\hline Code & LBI & JON & HAU & MER & TAU \\
\hline LBI & & $\mathbf{0 . 0 0 1 8}$ & 0.2370 & 0.2194 & 0.2752 \\
JON & $\mathbf{0 . 0 0 2 2}$ & & $\mathbf{0 . 0 0 0 0}$ & 0.0430 & 0.0361 \\
HAU & 0.0008 & $\mathbf{0 . 0 0 2 1}$ & & 0.0730 & $\mathbf{0 . 0 0 9 8}$ \\
MER & 0.0032 & 0.0024 & 0.0005 & & 0.3998 \\
TAU & 0.0013 & 0.0033 & $\mathbf{0 . 0 0 2 2}$ & 0.0004 & \\
\hline
\end{tabular}

Table 5.4 Pairwise FST values for Pecten novaezelandiae for the temporal study are below the diagonal and p-values are above the diagonal. Significant values are in bold after FDR testing $(\mathrm{p}<0.05)$. Locations abbreviations as per Table 5.1.

\begin{tabular}{ccccccc}
\hline Code & LBI12 & LBI14 & MER12 & MER14 & TAU12 & TAU14 \\
\hline LBI12 & & 0.1862 & 0.3279 & 0.2563 & 0.0627 & $\mathbf{0 . 0 2 5 6}$ \\
LBI14 & 0.0000 & & 0.0939 & 0.0337 & 0.2210 & 0.0457 \\
MER12 & 0.0007 & 0.0025 & & $\mathbf{0 . 0 1 9 8}$ & 0.2319 & 0.0501 \\
MER14 & 0.0000 & 0.0025 & $\mathbf{0 . 0 0 4 8}$ & & $\mathbf{0 . 0 1 5 2}$ & $\mathbf{0 . 0 0 5 7}$ \\
TAU12 & 0.0029 & 0.0010 & 0.0007 & $\mathbf{0 . 0 0 3 0}$ & & $\mathbf{0 . 0 1 3 5}$ \\
TAU14 & $\mathbf{0 . 0 0 1 7}$ & 0.0015 & 0.0015 & $\mathbf{0 . 0 0 0 8}$ & $\mathbf{0 . 0 0 0 5}$ & \\
\hline
\end{tabular}


For the spatial analyses, the NJ tree showed locations MER and TAU grouped together with $44 \%$ support and the locations JON and LBI grouped separately (Figure 5.4a). For the temporal analyses, the locations TAU12 and MER12 were grouped together (48\% bootstrap) as well as locations LBI12 and MER14 (54\% bootstrap). The location LBI14 was grouped separately (Figure 5.4b).

(a)

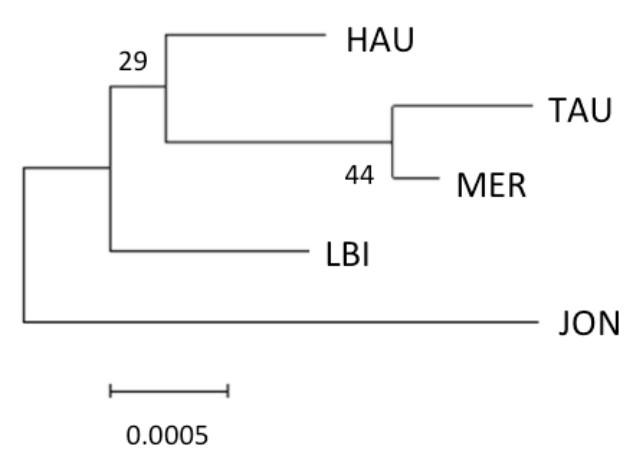

(b)

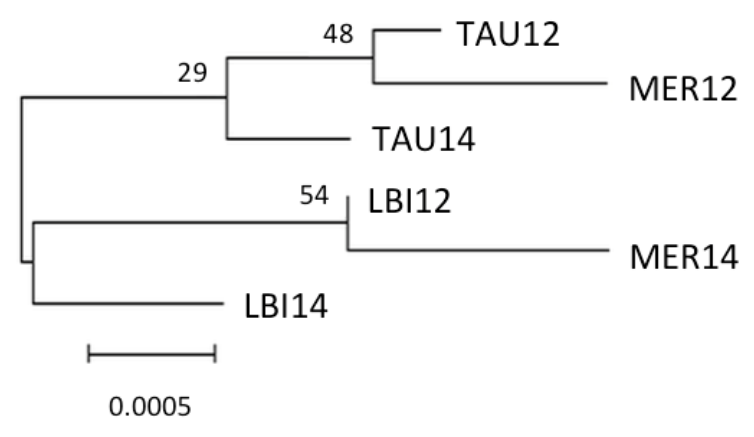

Figure 5.4 Neighbour joining population tree for Pecten novaezelandiae based on $\mathrm{F}_{\mathrm{ST}}$ values for (a) the spatial study and (b) the temporal study. Location abbreviations as per Table 5.1.

The principal components analysis (PCoA) for the spatial study showed that Axis 1 explained $63.96 \%$ of the variation and Axis 2 explained $23.25 \%$. The analysis grouped the locations TAU and MER together (Figure 5.5a). For the temporal analyses, the PCoA showed that Axis 1 explained 60.93\% of the variation and Axis 2 explained $25.47 \%$. The analysis grouped the locations TAU12 and MER12 on the left side of the axis, with the remaining locations on the right side of the axis (Figure 5.5b). 
(a)

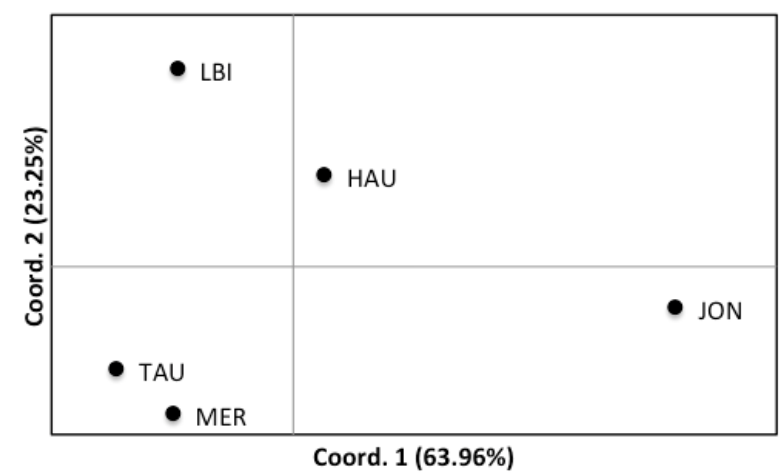

(b)

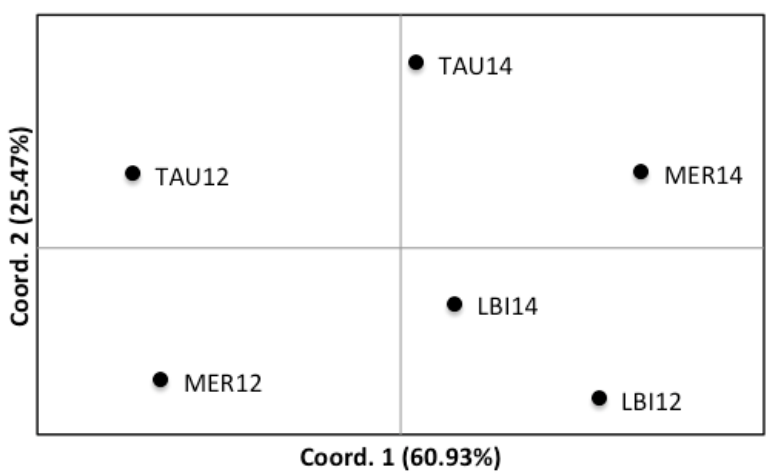

Figure 5.5 Principal components analysis (PCoA) for Pecten novaezelandiae using pairwise genetic distance for (a) the spatial and (b) the temporal study. Locations abbreviations as per Table 5.1.

For the spatial study, the non-parametric analysis (AWclust) showed a large gap value at $\mathrm{k}=3$, with some degree of overlap of confidence intervals (Figures 5.6a and 5.7). For the temporal study, the largest gap value of AWclust analyses was at $\mathrm{k}=6$, with extensive overlap of confidence intervals (Figures 5.6b and 5.8).

(a)

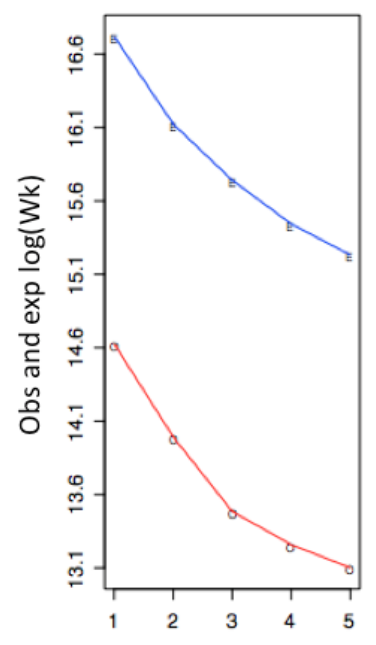

Number of clusters $\mathrm{K}$

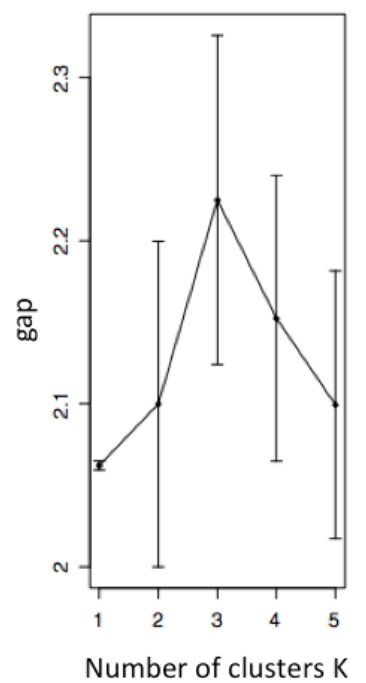

(b)

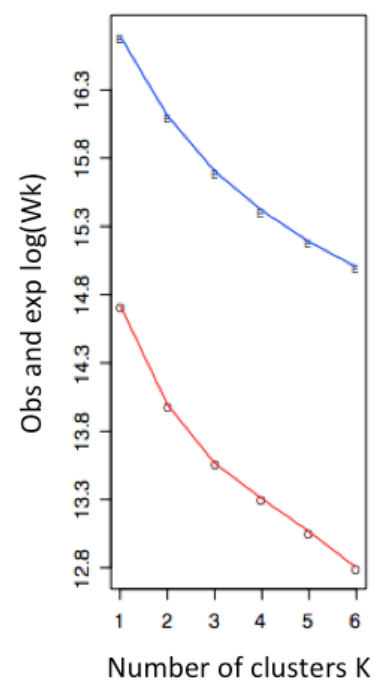

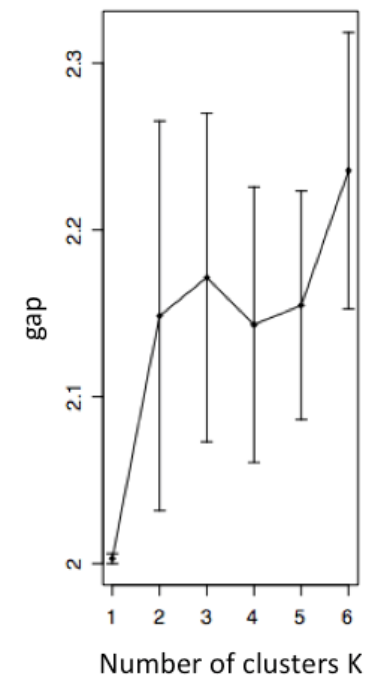

Figure 5.6 AWclust analyses for Pecten novaezelandiae for (a) the spatial study and (b) the temporal study. 


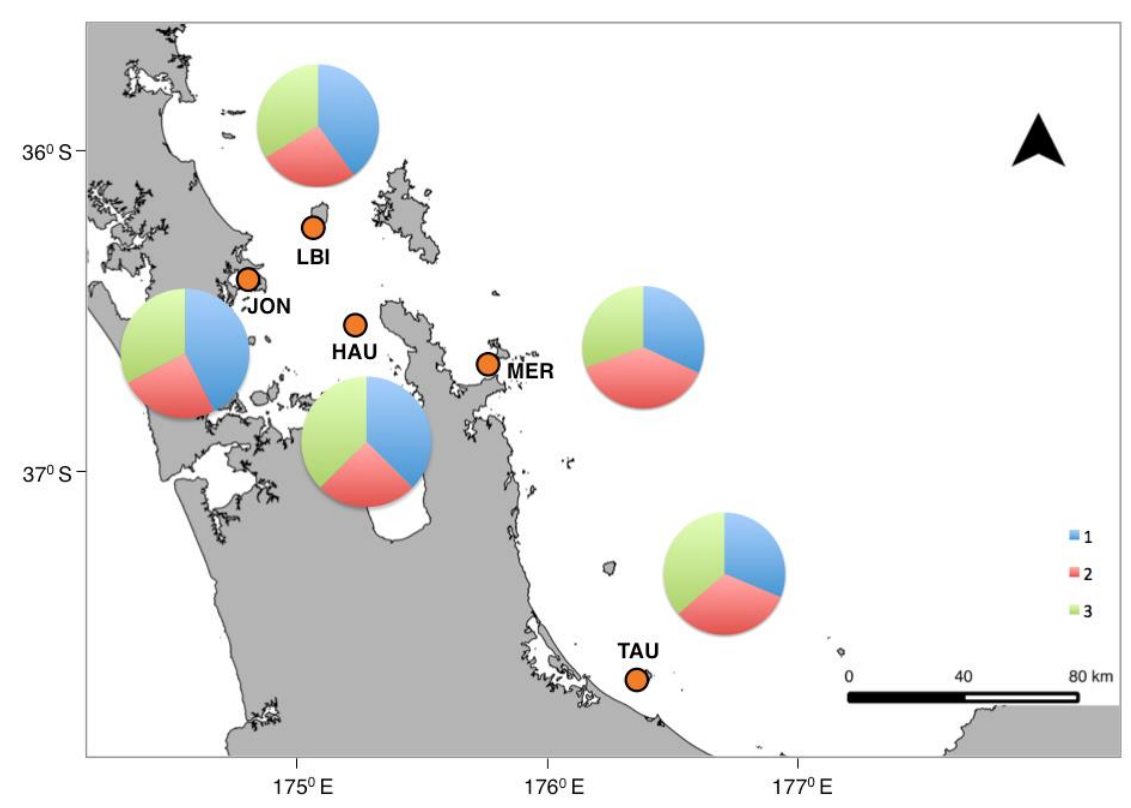

Figure 5.7 AWclust analysis results for Pecten novaezelandiae for the spatial study. Each colour represents an estimated portion of $\mathrm{K}=3$ clusters. Locations abbreviations as per Table 5.1.

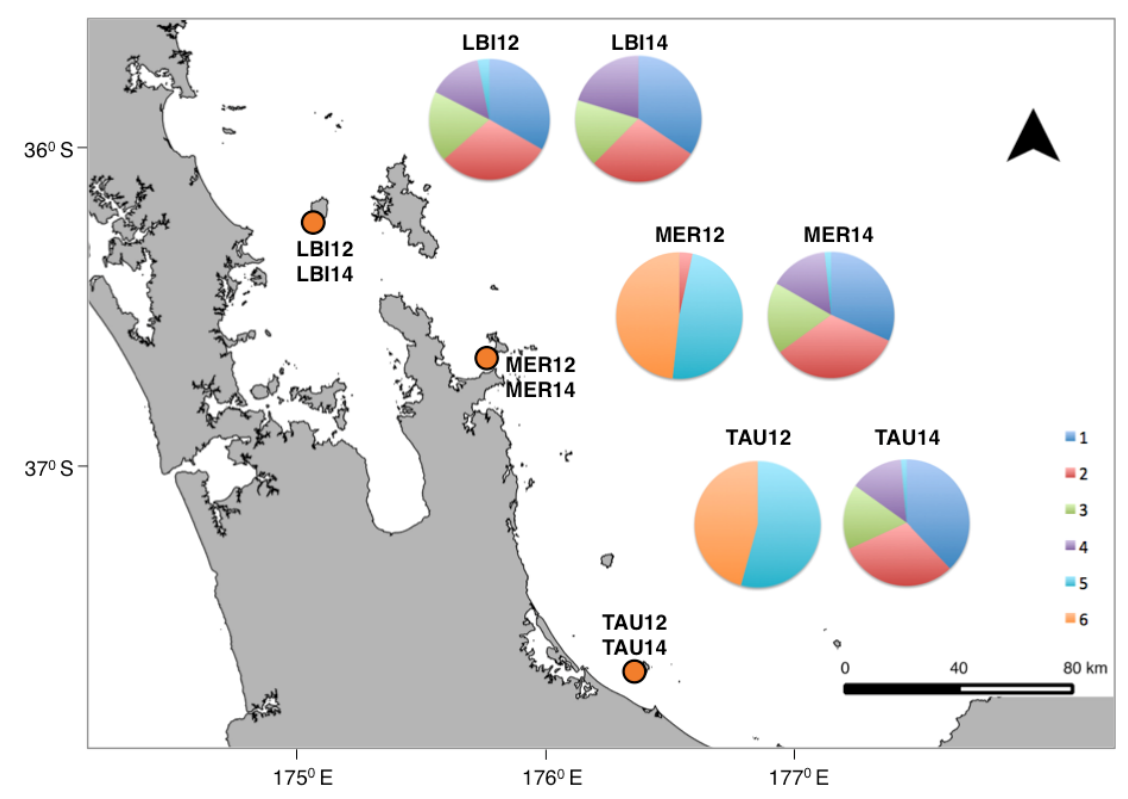

Figure 5.8 AWclust analysis results for Pecten novaezelandiae for the temporal study. Each colour represents an estimated portion of $\mathrm{K}=6$ clusters. Locations abbreviations as per Table 5.1.

The Bayesian analysis (Structure) showed the greatest value of $\Delta \mathrm{K}$ for 4 clusters for the spatial study (Table 5.5a) and 2 clusters for the temporal study (Table 5.5b). However, the 
maximum log-likelihood of $\mathrm{K}$ showed the greatest value of 1 cluster (no structure, panmixia) for both studies and the bar plot did not show any trend of proportions of clusters (results not shown).

Table 5.5 Evanno table (Evanno et al. 2005) output for (a) the spatial study and (b) the temporal study of genetic structure of Pecten novaezelandiae.

(a)

$\begin{array}{ccccccc}\mathbf{K} & \text { Reps } & \text { Mean LnP(K) } & \text { Stdev LnP(K) } & \operatorname{Ln}^{\prime}(\mathbf{K}) & |\mathbf{L n} "(\mathbf{K})| & \text { Delta K } \\ 1 & 5 & -14452.900000 & 0.484768 & - & - & - \\ 2 & 5 & -14669.660000 & 93.725226 & -216.760000 & 41.400000 & 0.441717 \\ 3 & 5 & -14927.820000 & 199.987517 & -258.160000 & 67.060000 & 0.335321 \\ 4 & 5 & -15118.920000 & 148.555737 & -191.100000 & 419.060000 & 2.820894 \\ 5 & 5 & -15729.080000 & 485.813531 & -610.160000 & - & -\end{array}$

(b)

\begin{tabular}{ccccccc} 
K & Reps & Mean LnP(K) & Stdev LnP(K) & Ln'(K) & $\mid$ Ln'(K)| & Delta K \\
\hline 1 & 5 & -12477.500000 & 1.614001 & - & - & - \\
2 & 5 & -12532.900000 & 26.360861 & -55.400000 & 59.760000 & 2.266997 \\
3 & 5 & -12648.060000 & 72.634172 & -115.160000 & 69.660000 & 0.959053 \\
4 & 5 & -12693.560000 & 123.017511 & -45.500000 & 192.760000 & 1.566931 \\
5 & 5 & -12931.820000 & 209.378478 & -238.260000 & 6.280000 & 0.029994 \\
6 & 5 & -13163.800000 & 325.368875 & -231.980000 & - & -
\end{tabular}

The analyses of molecular variance (AMOVA) for the spatial study using 3 regions showed that $0.3 \%$ of the variation was explained among regions and the models were significant at $\mathrm{p}<0.01$ (Table 5.6). AMOVA analysis using 4 regions showed that $0.4 \%$ of the variation was explained among regions and the models were significant at $\mathrm{p}<0.01$ (Table 5.7). 
Table 5.6 Analysis of molecular variance (AMOVA) for the spatial study of $P$. novaezelandiae using 3 regions.

\begin{tabular}{lllllll}
\hline $\begin{array}{l}\text { Source of } \\
\text { variation }\end{array}$ & $\begin{array}{l}\text { Degrees } \\
\text { of } \\
\text { Freedom }\end{array}$ & $\begin{array}{l}\text { Sum of } \\
\text { Squares }\end{array}$ & $\begin{array}{l}\text { Estimated } \\
\text { Variance }\end{array}$ & $\begin{array}{l}\% \\
\text { Variation }\end{array}$ & $\begin{array}{l}\text { Differentiation } \\
\text { Indices }\end{array}$ & Significance \\
\hline $\begin{array}{l}\text { Among } \\
\text { Regions }\end{array}$ & 2 & 13.94 & 0.011 & $0.3 \%$ & $\mathrm{~F}_{\mathrm{RT}}=0.003$ & 0.002 \\
$\begin{array}{l}\text { Among } \\
\text { Locations }\end{array}$ & 2 & 9.16 & 0.004 & $0.1 \%$ & $\mathrm{~F}_{\mathrm{SR}}=0.001$ & 0.133 \\
$\begin{array}{l}\text { Among } \\
\text { Individuals }\end{array}$ & 397 & 1557.23 & 0.512 & $15.0 \%$ & $\mathrm{~F}_{\mathrm{ST}}=0.004$ & 0.001 \\
$\begin{array}{l}\text { Within } \\
\text { Locations }\end{array}$ & 402 & 1165.00 & 2.898 & $84.6 \%$ & $\mathrm{~F}_{\mathrm{IS}}=0.150$ & 0.001 \\
\hline Total & 803 & 2745.34 & 3.425 & $100.0 \%$ & $\mathrm{~F}_{\mathrm{IT}}=0.154$ & 0.001 \\
\hline
\end{tabular}

Table 5.7 Analysis of molecular variance (AMOVA) for the spatial study of $P$. novaezelandiae using 4 regions.

\begin{tabular}{lllllll}
\hline $\begin{array}{l}\text { Source of } \\
\text { variation }\end{array}$ & $\begin{array}{l}\text { Degrees } \\
\text { of } \\
\text { Freedom }\end{array}$ & $\begin{array}{l}\text { Sum of } \\
\text { Squares }\end{array}$ & $\begin{array}{l}\text { Estimated } \\
\text { Variance }\end{array}$ & $\begin{array}{l}\% \\
\text { Variation }\end{array}$ & $\begin{array}{l}\text { Differentiation } \\
\text { Indices }\end{array}$ & Significance \\
\hline $\begin{array}{l}\text { Among } \\
\text { Regions }\end{array}$ & 3 & 19.35 & 0.014 & $0.4 \%$ & $\mathrm{~F}_{\mathrm{RT}}=0.004$ & 0.001 \\
$\begin{array}{l}\text { Among } \\
\text { Locations }\end{array}$ & 1 & 3.75 & 0.000 & $0.0 \%$ & $\mathrm{~F}_{\mathrm{SR}}=0.000$ & 0.544 \\
$\begin{array}{l}\text { Among } \\
\text { Individuals }\end{array}$ & 397 & 1557.23 & 0.512 & $15.0 \%$ & $\mathrm{~F}_{\mathrm{ST}}=0.004$ & 0.001 \\
$\begin{array}{l}\text { Within } \\
\text { Locations }\end{array}$ & 402 & 1165.00 & 2.898 & $84.6 \%$ & $\mathrm{~F}_{\mathrm{IS}}=0.150$ & 0.001 \\
\hline Total & 803 & 2745.34 & 3.425 & $100.0 \%$ & $\mathrm{~F}_{\mathrm{IT}}=0.154$ & 0.001 \\
\hline
\end{tabular}

For the temporal study, AMOVA analysis using all locations grouped in 2 regions (between years) showed that $0.02 \%$ of the variation was explained among regions and the model was not significant (Table 5.8). AMOVA analysis using locations MER and TAU grouped in 2 regions (between years) showed that $0.23 \%$ of the variation was explained among regions and the model was significant at $\mathrm{p}<0.05$ (Table 5.9). 
Table 5.8 Analysis of molecular variance (AMOVA) for the temporal study of $P$. novaezelandiae using all locations grouped in 2 regions (between years).

\begin{tabular}{lllllll}
\hline $\begin{array}{l}\text { Source of } \\
\text { variation }\end{array}$ & $\begin{array}{l}\text { Degrees } \\
\text { of } \\
\text { Freedom }\end{array}$ & $\begin{array}{l}\text { Sum of } \\
\text { Squares }\end{array}$ & $\begin{array}{l}\text { Estimated } \\
\text { Variance }\end{array}$ & $\begin{array}{l}\% \\
\text { Variation }\end{array}$ & $\begin{array}{l}\text { Differentiation } \\
\text { Indices }\end{array}$ & Significance \\
\hline Among & 1 & 4.72 & 0.001 & $0.02 \%$ & $\mathrm{~F}_{\mathrm{RT}}=0.000$ & 0.319 \\
$\begin{array}{l}\text { Regions } \\
\text { Among }\end{array}$ & & 17.70 & 0.004 & $0.13 \%$ & $\mathrm{~F}_{\mathrm{SR}}=0.001$ & 0.086 \\
$\begin{array}{l}\text { Locations } \\
\text { Among }\end{array}$ & 4 & 1455.59 & 0.473 & $13.94 \%$ & $\mathrm{~F}_{\mathrm{ST}}=0.002$ & 0.042 \\
$\begin{array}{l}\text { Individuals } \\
\text { Within }\end{array}$ & 377 & 1116.50 & 2.915 & $85.91 \%$ & $\mathrm{~F}_{\mathrm{IS}}=0.140$ & 0.001 \\
\hline Locations & 383 & 2594.50 & 3.393 & $100.0 \%$ & $\mathrm{~F}_{\mathrm{IT}}=0.141$ & 0.001 \\
\hline Total & 765 & & & & & \\
\hline
\end{tabular}

Table 5.9 Analysis of molecular variance (AMOVA) for the temporal study of $P$. novaezelandiae using locations MER and TAU grouped in 2 regions (between years).

\begin{tabular}{lllllll}
\hline $\begin{array}{l}\text { Source of } \\
\text { variation }\end{array}$ & $\begin{array}{l}\text { Degrees } \\
\text { of } \\
\text { Freedom }\end{array}$ & $\begin{array}{l}\text { Sum of } \\
\text { Squares }\end{array}$ & $\begin{array}{l}\text { Estimated } \\
\text { Variance }\end{array}$ & $\begin{array}{l}\% \\
\text { Variation }\end{array}$ & $\begin{array}{l}\text { Differentiation } \\
\text { Indices }\end{array}$ & Significance \\
\hline $\begin{array}{l}\text { Among } \\
\text { Regions }\end{array}$ & 1 & 5.88 & 0.008 & $0.23 \%$ & $\mathrm{~F}_{\mathrm{RT}}=0.002$ & 0.026 \\
$\begin{array}{l}\text { Among } \\
\text { Locations }\end{array}$ & 2 & 7.77 & 0.000 & $0.01 \%$ & $\mathrm{~F}_{\mathrm{SR}}=0.000$ & 0.406 \\
$\begin{array}{l}\text { Among } \\
\text { Individuals }\end{array}$ & 252 & 966.93 & 0.452 & $13.31 \%$ & $\mathrm{~F}_{\mathrm{ST}}=0.002$ & 0.022 \\
$\begin{array}{l}\text { Within } \\
\text { Locations }\end{array}$ & 256 & 751.00 & 2.934 & $86.45 \%$ & $\mathrm{~F}_{\mathrm{IS}}=0.133$ & 0.001 \\
\hline Total & 511 & 1731.50 & 3.394 & $100.00 \%$ & $\mathrm{~F}_{\mathrm{IT}}=0.136$ & 0.001 \\
\hline
\end{tabular}

\section{Estimates of migration}

First-generation of migrants analysis detected a low number of migrants for all locations, for both the spatial and temporal studies ( 6 and 11 first-generation migrants, respectively; Tables 5.10, 5.12). This low number was probably an underestimation of first-generation migrants due to low levels of differentiation between locations as a result of very similar genotypes between migrants and individuals from the original locality. For the spatial study, the location with the highest level of correct assignment was HAU (75.8\%), while 
the location with the lowest level was TAU (26.3\%). These locations also had the lowest (HAU) and highest (TAU) percentage of individuals recruited from locations other than the collection site (Table 5.11).

Table 5.10 Number of first generation (F0) migrants detected for Pecten novaezelandiae at each sampled location for the spatial study in Coromandel. Locations abbreviations as per Table 5.1.

\begin{tabular}{|c|c|c|c|c|c|c|c|}
\hline \multicolumn{8}{|c|}{ Source of $F_{0}$ migrants } \\
\hline & & LBI & JON & HAU & MER & TAU & Total $\mathrm{F}_{0}$ migrants \\
\hline \multirow{5}{*}{ 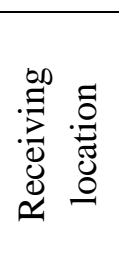 } & LBI & & & & & & 0 \\
\hline & JON & & & & 2 & & 2 \\
\hline & HAU & & 1 & & 1 & & 2 \\
\hline & MER & & & & & & 0 \\
\hline & TAU & 1 & 1 & & & & 2 \\
\hline
\end{tabular}

Table 5.11 Percentage of individuals of Pecten novaezelandiae collected from each sampling location, assigned to each sampled potential source location for the spatial study in the Coromandel. "Total other regions" is the percentage of individuals recruited from regions other than the collection site. Location abbreviations as per Table 5.1.

\begin{tabular}{|c|c|c|c|c|c|c|c|c|}
\hline & \multicolumn{7}{|c|}{ Assigned location } & \multirow[t]{2}{*}{$\begin{array}{c}\text { Total othe } \\
\text { location }\end{array}$} \\
\hline & & LBI & JON & HAU & MER & TAU & Unassigned & \\
\hline \multirow{5}{*}{ 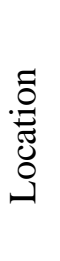 } & LBI & 55.8 & 6.3 & 28.4 & 4.2 & 5.3 & & 44.2 \\
\hline & JON & 20.0 & 50.0 & 20.0 & 7.5 & 2.5 & & 50.0 \\
\hline & HAU & 10.1 & 6.1 & 75.8 & 4.0 & 4.0 & & 24.2 \\
\hline & MER & 15.7 & 10.0 & 28.6 & 40.0 & 5.7 & & 60.0 \\
\hline & TAU & 23.2 & 10.1 & 34.3 & 5.1 & 26.3 & 1.0 & 72.7 \\
\hline
\end{tabular}


Table 5.12 Number of first generation (F0) migrants detected for Pecten novaezelandiae at each sampled location for the temporal study in the Coromandel. Location abbreviations as per Table 5.1.

\begin{tabular}{|c|c|c|c|c|c|c|c|c|}
\hline \multicolumn{9}{|c|}{ Source of F0 migrants } \\
\hline & & LBI12 & LBI14 & MER12 & MER14 & TAU12 & TAU14 & $\begin{array}{l}\text { Total F0 } \\
\text { migrants }\end{array}$ \\
\hline \multirow{6}{*}{ 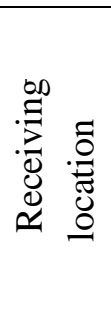 } & LBI12 & & & & 1 & & 1 & 2 \\
\hline & LBI14 & & & & & & & 0 \\
\hline & MER12 & & & & & 1 & & 1 \\
\hline & MER14 & 1 & & 1 & & 1 & & 3 \\
\hline & TAU12 & & & & & & 1 & 1 \\
\hline & TAU14 & 1 & & 2 & 1 & & & 4 \\
\hline
\end{tabular}

For the temporal study, the location with the highest level of correct assignment was LBI12 (71.4\%), while the location with the lowest level was LBI14 (40.6\%). These locations also had the lowest (LBI12) and highest (LBI14) percentage of individuals recruited from locations other than the collection site (Table 5.13).

Table 5.13 Percentage of individuals of Pecten novaezelandiae collected from each sampling location, assigned to each sampled potential source location for the temporal study in the Coromandel. "Total other regions" is the percentage of individuals recruited from regions other than the collection site. Location abbreviations as per Table 5.1.

\begin{tabular}{|c|c|c|c|c|c|c|c|c|c|}
\hline & & & & Assį & led locatic & & & & $\begin{array}{l}\text { Total } \\
\text { other }\end{array}$ \\
\hline & & LBI12 & LBI14 & MER12 & MER14 & TAU12 & TAU14 & Unassigned & \\
\hline & LBI12 & 71.4 & 1.6 & 9.5 & 3.2 & 0.0 & 14.3 & & 28.6 \\
\hline & LBI14 & 31.2 & 40.6 & 12.5 & 7.8 & 1.6 & 6.3 & & 59.4 \\
\hline ธี & MER12 & 13.3 & 1.7 & 65.0 & 6.7 & 5.0 & 8.3 & & 35.0 \\
\hline ت & MER14 & 20.0 & 3.3 & 21.7 & 45.0 & 1.7 & 8.3 & & 55.0 \\
\hline 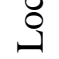 & TAU12 & 27.1 & 2.9 & 10.0 & 4.3 & 48.6 & 7.1 & & 51.4 \\
\hline & TAU14 & 12.1 & 6.1 & 15.2 & 9.1 & 4.5 & 53.0 & & 47.0 \\
\hline
\end{tabular}




\section{Relatedness}

Overall, mean relatedness ranged from -0.019 in TAU14 to 0.017 in MER14. All locations had the means within the upper and lower 95\% confidence intervals of randomly generated values (Figure 5.9) and no location was significantly different from random.

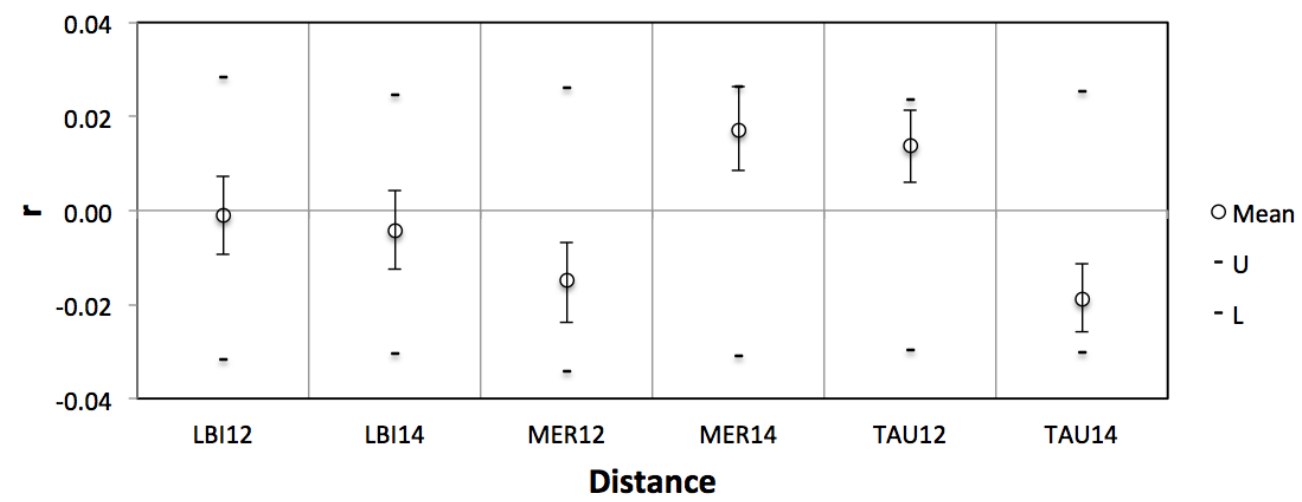

Figure 5.9 Mean values of observed relatedness for Pecten novaezelandiae for the temporal study. Upper and lower error bars represent the $95 \%$ confidence interval about the mean values as determined by 1000 bootstraps resampling. Upper (U) and lower (L) confidence limits represent the $95 \%$ confidence interval for the null hypothesis of No Difference' across the locations as determined by 999 permutations. Location abbreviations as per Table 5.1.

\section{Oceanographic model}

The connectivity estimation showed a clear pattern of oceanic currents flowing predominantly southeast for all scenarios. The highest percentage of propagules (20\%) dispersed less than a few tens of kilometres for scenario (a) when particles were released at Little Barrier Island (Figure 5.10a). However, for scenarios (b) and (c) when particles were released at Mercury Island and Tauranga, the edge of the $20 \%$ dispersal kernel was not evident (Figure 5.10b, c). The dispersal kernel of low probability (the blue area) extended a few hundred kilometres from the release point of particles for all scenarios (illustrated in Figure 5.10a-c). 


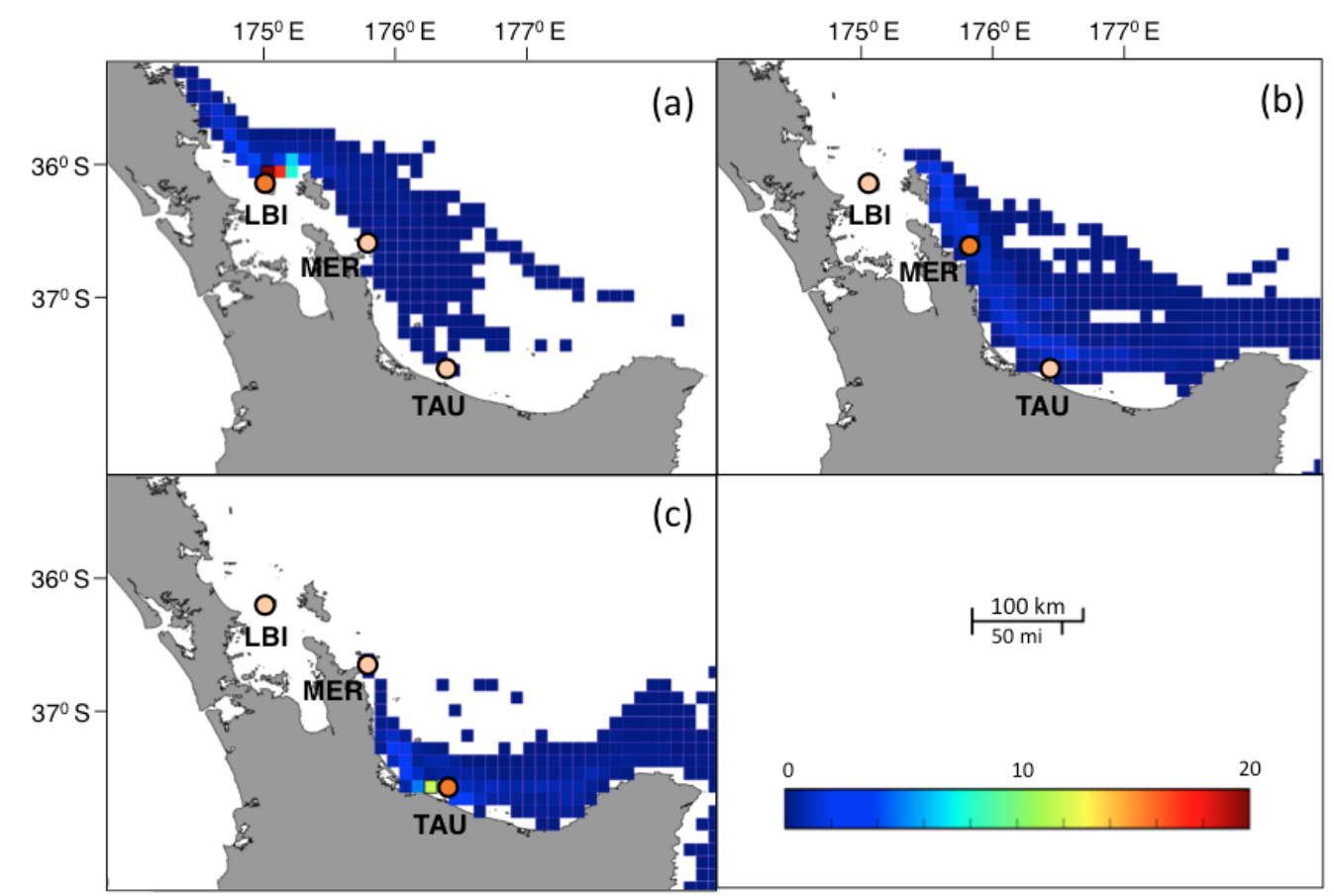

Figure 5.10 Probability distributions of particles released at (a) Little Barrier Island (LBI), (b) Mercury Island (MER) and (c) Tauranga (TAU). Maps show results of particles with diel vertical migration run for 25 days and released in early summer. Results are averaged across ten years (1997-2007). The colour bar indicates final position probability (\%). Location abbreviations as per Table 5.1.

\section{Discussion}

Information on spatial and temporal genetic structure is critical for identifying meaningful fisheries management units. Failure to incorporate this information can result in reduced productivity, local reduction of populations or even extinction of local populations (Worm et al. 2006). Therefore, studies like this one are important to understand the spatial and temporal dynamics of larval recruitment for informing fisheries management. This study shows small but significant spatial and temporal genetic differentiation in the Coromandel region, suggesting that the sampled locations did not form a single panmictic unit with free gene flow. The levels of genetic variation are consistent with the findings of chapter 3, as very low genetic differentiation was found within regional clusters and levels of differentiation were lower at a smaller spatial scale. 


\section{Genetic diversity}

Levels of genetic diversity for both spatial and temporal studies were in agreement with Chapter 3 and with other scallop species (Kenchington et al. 2006; Zhan et al. 2009; Arias et al. 2010; Hemond \& Wilbur 2011; Marín et al. 2012). Genetic diversity in terms of allelic richness showed a decrease from 2012 to 2014 and this trend was also observed for measures of private allelic richness. In addition, there was a temporal increase in inbreeding rates $\left(F_{I S}\right)$ for all locations.

A possible explanation for these observations is that these locations correspond to three very important fishing beds (LBI, MER and TAU corresponding to fishery areas of "Barrier", "Mercury" and "Plenty", respectively; Ministry of Primary Industries 2013), so the effects of fishing can be reflected in the temporal genetic pattern. Other studies have shown a significant loss of genetic diversity in overfished populations (Hauser et al. 2002; Pinsky \& Palumbi 2014) indicating that not only can very small populations suffer significant loss of genetic diversity but also many abundant marine species may have their evolutionary potential impacted by overfishing. On the other hand, a rapid decrease in genetic diversity, particularly for the locations MER and TAU, can be related to changes in larval source as a result of stochastic oceanographic conditions or to temporal variability in recruitment success.

\section{Population structure}

The different analyses of population structure showed evidence of a low but significant genetic spatial and temporal structure in the Coromandel region. The clustering analyses were unable to identify clear patterns of genetic structure, which is not surprising given the low levels of genetic differentiation. Although pairwise $\mathrm{F}_{\mathrm{ST}}$ values were very low $(0.0004$ to 0.0048), neighbor joining analysis, PCoA and AMOVA supported the $F_{S T}$ values. In addition, even low migration rates that suggest demographic independence can coincide with very small FST values (Waples et al. 2008).

This study provided evidence of moderate levels of self-recruitment of Pecten novaezelandiae at Little Barrier Island (LBI). For the temporal study, estimates of migration detected a high percentage of individuals self-assigned to LBI12 (71.4\%), while 
the location LBI14 had a high percentage of individuals assigned to both LBI12 and LBI14 (71.8\%). In addition, the oceanographic model supported the results of estimates of migration since the first scenario (particles released in LBI) produced the highest probability values of particle dispersal $(20 \%)$ at less than a few tens of kilometres from LBI. This dispersal kernel of higher probability was not observed in the two other scenarios (particles released in MER and TAU), which is also in agreement with estimates of migration suggesting that these locations have lower levels of self-recruitment as a result of the predominant ocean currents.

This study also suggested moderate to high levels of self-recruitment for locations JON and HAU as indicated by estimates of migration. These observations are in agreement with the prevailing ocean currents in the region. Although a high-resolution hydrodynamic model more suitable for simulations in the inner part of the Hauraki Gulf was not available for this study, ocean currents are expected to become slower in the inner part of the Gulf (Zeldis et al. 2004) and consequently to reduce further the potential for larval dispersal. As a comparison, there is evidence of potential of gene flow between locations MER and TAU, where ocean currents are expected to be faster (i.e., provide more connectivity). These locations were not differentiated, as they had the lowest pairwise F $_{\text {ST }}$ value and similar genetic diversity and temporal structure. As the simulation results show for the temporal study, ocean currents outside the Hauraki Gulf can facilitate gene flow between locations MER and TAU.

Interestingly, the location HAU had the highest levels of self-recruitment (75.8\%) and also a high percentage of individuals from other locations were recruited from HAU (between $20 \%$ in JON and $34.3 \%$ in TAU). It is unclear if these patterns represent accurate gene flow estimates or if they are an artefact of the analysis. Studies suggest that assignment tests can be very useful for population studies and a good indicator of gene flow between populations but their accuracy is highly dependent on the levels of population differentiation. For example, Berry et al. (2004) showed that assignment methods were $100 \%$ accurate for low to moderate levels of genetic differentiation $\left(F_{\mathrm{ST}} \sim 0.07\right)$ but the accuracy was reduced to $\sim 78 \%$ for populations with lower levels of genetic subdivision $\left(F_{\mathrm{ST}}=0.04\right)$. Therefore, results from this study need to be interpreted with caution because of the limitations of assignment tests in measuring dispersal of $P$. novaezelandiae, which has little population differentiation in the Coromandel region. Although population 
differentiation was low, both assignment tests and the PCoA analysis supported the observed levels of differentiation between locations. In addition, for the temporal analysis, the patterns of migration were also in agreement with the oceanographic model, providing some confidence that assignment tests can reasonably represent dispersal levels across sampled locations. If this is true, the location HAU can coincide with an important source population. Lastly, it has been shown for Pecten novaezelandiae that adults in deeper beds (28-40 m) spawn first (Tunbridge 1968). As the sampled location HAU coincides with the deepest bed of scallops in the Coromandel region, adults in this population can be spawning earlier in the season and therefore are more likely to occupy habitats at settlement locations early in the season.

The temporal stability in LBI, moderate self-recruitment and the oceanographic model (first scenario particles released in LBI) represent evidence that this location might be a source population. A temporally stable genetic structure in an area indicates either a consistent source of recruits or a consistent system of retention (Larson \& Julian 1999). As locations north of LBI were not sampled for this study, the former cannot be tested. However, the oceanographic model showed a clear pattern of oceanic currents flowing southeast indicating that possibly locations north of LBI can also be a source of recruits. In addition, the temporally stable genetic structure is possibly also related to a consistent system of retention, as indicated by the oceanographic model, assignment tests and mean relatedness values.

For the locations MER and TAU, the high percentage of individuals $(60 \%$ and $72.7 \%$ for MER and TAU, respectively) was recruited from locations other than the collection site and assignment tests for the temporal study and the oceanographic model suggest that these locations are sink populations. In addition, the lack of temporal stability indicates temporal changes in larval source at these two locations, which can be the result of a combination of processes such as 1) stochastic oceanographic conditions, 2) low levels of self-recruitment and 3) temporal variability in recruitment success.

Temporal instability patterns of genetic structure as a result of variable source of recruits have been observed for other marine species (Barber et al. 2002; Robainas-Barcia et al. 2008; Hogan et al. 2010; Kesäniemi et al. 2014; Yednock \& Neigel 2014). Ocean currents can greatly affect the movement of pelagic larvae even at small scales (Pringle \& Wares 2007; Wood \& Gardner 2007) and the population genetic structure of some marine species 
can correspond with ocean circulation (White et al. 2010). For example, a small-scale genetic structure has been observed for the Zhikong scallop Chlamys farreri and locations separated by strong marine currents or within different marine gyres showed significant genetic differentiation (Zhan et al. 2009). Changes in larval source can also be the result of low levels of self-recruitment. For example, Saenz-Agudelo et al. (2012) showed that the coral reef fish Amphiprion polymnus had lower levels of self-recruitment at the scale of individual sites, which was related to higher levels of connectivity between these sites. This is in agreement with the results of this study, as the locations MER and TAU had the lowest levels of self-recruitment and highest potential for gene flow. Since migrants' recruitment is greater than local self-recruitment, a change in recruits migrating from a different genetic source could contribute to a lack of temporal stability in these locations. Finally, variance in reproductive success in which only a few individuals contribute to the next generation has been shown to affect the temporal genetic structure in some marine species (Flowers et al. 2002; Christie et al. 2010; Underwood et al. 2012; Pusack et al. 2014). The small-scale spatial and temporal genetic structure observed for the Atlantic sea scallop (Placopecten magellanicus) was possibly explained by inter-annual differences in larval supply or reproductive success (Owen \& Rawson 2013). Although the variance in reproductive success cannot be tested in this study, it can be one of the processes influencing the genetic structure of Pecten novaezelandiae and can also help explain the significant between-year differences in allele frequencies found for locations MER and TAU.

\section{Fisheries management implications}

The expected relationships between locations sampled in the Coromandel region are summarised in Figure 5.11. The sampled locations MER and TAU coincide with the very productive fishing areas of "Mercury" and "Plenty", respectively, that have high levels of annual recruitment, particularly the Mercury Islands (Ministry of Primary Industries 2013). The results of this study suggest that oceanographic mechanisms in these regions do not facilitate high levels of self-recruitment, which implies that larvae recruiting to these areas are mostly coming from other sources. 


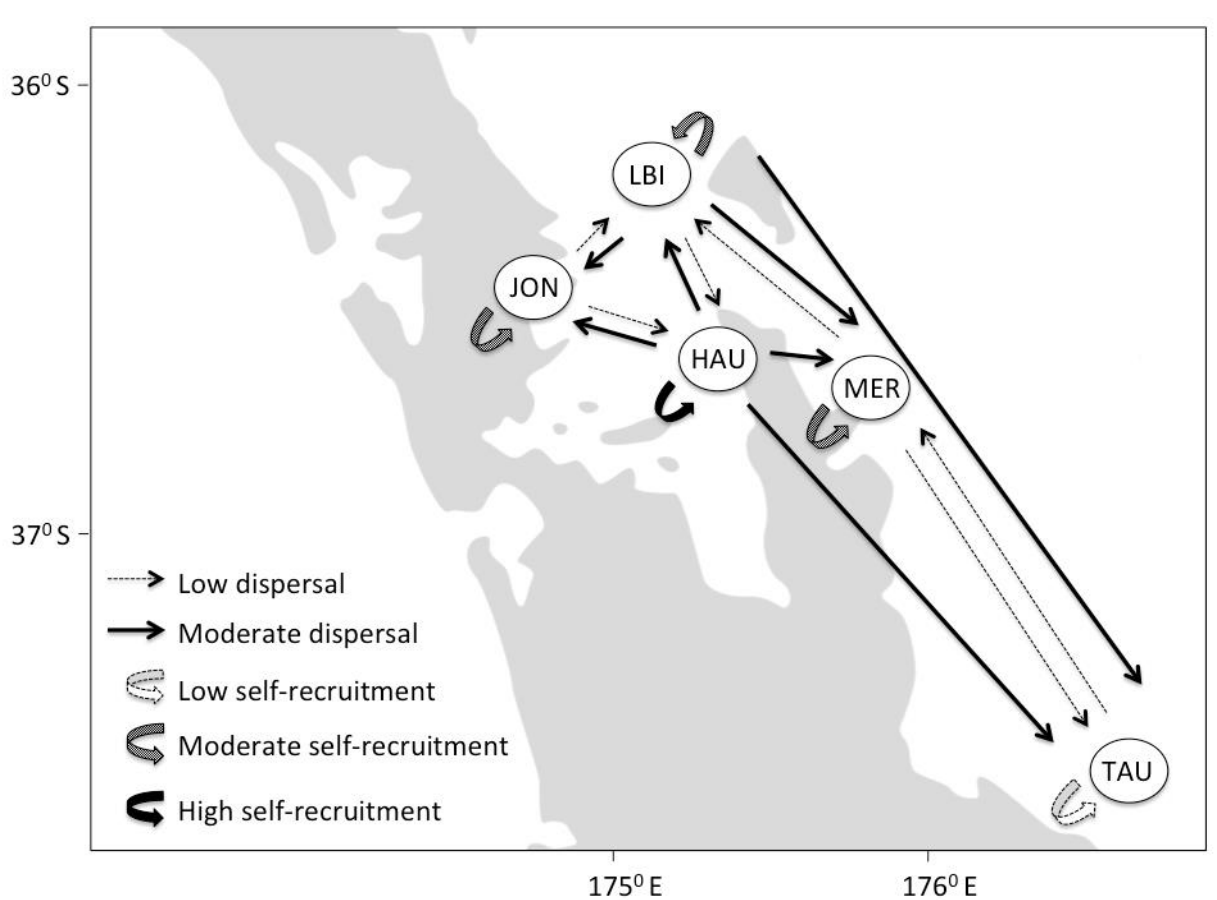

Figure 5.11 Expected relationships between locations sampled in Coromandel with estimated levels of larval dispersal and self-recruitment (low, moderate and high) indicated by the arrows. Circles are locations sampled, site abbreviations as per Table 5.1.

The genetic analyses and the oceanographic model suggest that the location LBI can be one of the sources of larvae recruiting to MER and TAU. This location (LBI) coincides with the very productive fishery area of Little Barrier Is (Ministry of Primary Industries 2013a). In addition, the genetic data also showed a stronger pattern of a source population for the location HAU. This location coincides with a recently found fishery area with good densities of scallops at 45-50 m depth that supported a large proportion of fishing in 2011 and 2012 (Ministry of Primary Industries 2013a). Therefore, as important source populations, management of these fisheries should be cautious. However, as levels of genetic differentiation between locations were very low, there are limitations in the assignment analyses and these results should be interpreted with caution. A high-resolution hydrodynamic model more suitable for simulations in the inner part of the Hauraki Gulf would help deciphering the complex nature of larval dispersal of Pecten novaezelandiae. Also, other source/sink populations can potentially be present in the region but were not sampled and are not represented in this study. 
The source-sink population dynamics detected in this study have important implications for fisheries management. For example, adults in the sink populations (MER and TAU) are less likely to contribute to the larval pool and an attempt to restock these populations would probably not be as successful as restocking source populations. Restocking of populations through translocation from sink to source populations has been successfully attempted for the Tasmanian rock lobster Jasus edwardsii (Gardner \& Van Putten 2008). Translocations can complement the management of marine resources but the creation of marine protected areas (MPAs) capable of both self-recruitment and exporting larvae to other unprotected and exploited areas is also an important tool for fisheries management. Comparing to no spatial management, strategically placed MPAs can for example substantially increase profits (Rassweiler et al. 2012) and also abundance and size of organisms (Pande et al. 2008).

This study shows complex spatial and temporal genetic patterns of $P$. novaezelandiae in the Coromandel region. The physical oceanographic characteristics likely play a significant role in generating these patterns of differentiation, which highlights how important it is to incorporate multidisciplinary approaches to understand marine population dynamics. However, this has yet to be widely applied in New Zealand and there is still a lack of empirical data using multidisciplinary tools that support connectivity patterns. This study provided important information for management and conservation applications of the New Zealand scallop. Patterns of dispersal and population dynamics have important effects on the genetic composition and resilience of populations. This study is the first step towards a better understanding of patterns of marine connectivity and dynamics of populations. 
CHAPTER 6

General discussion 
The New Zealand marine environment is a very complex and diverse system, which represents a major management and conservation challenge (Gordon et al. 2010). At the same time, it is an ideal system for studying the processes shaping the genetic structure of marine species. The increasing exploitation of marine resources and human-induced environmental changes pose a threat to the world's oceans and information on the genetic structure and population dynamics of species is increasingly important for the effective management and conservation of marine resources. Population genetics integrated with ecological data is a powerful approach to address questions about population structure and dynamics of marine organisms.

The general aims of this thesis were to investigate the genetic structure of Pecten novaezelandiae and its relationship with environmental variation around New Zealand, and also to examine spatial and temporal patterns of dispersal of $P$. novaezelandiae in the Coromandel fishery region. For this first study of population genetics of $P$. novaezelandiae, a multidisciplinary approach was used with genetic markers (specifically developed for this study), environmental data and hydrodynamic modelling. Little is known about population dynamics of the New Zealand scallop, and species inhabiting estuarine and sandy open coast habitats in general (Gardner et al. 2010; Constable 2014; Hannan 2014). Therefore, this study provides important information for management of $P$. novaezelandiae, contributes to a broader understanding of the patterns of gene flow in the New Zealand marine environment and brings important information about the processes shaping the patterns of population genetic structure.

In this general discussion the major findings of this study are first reviewed, with chapters 2, 3, 4 and 5 being addressed separately. Evolutionary consequences that arise from this research are discussed and implications for the management of $P$. novaezelandiae throughout its distribution range and in particular in the Coromandel fishery are then presented. Finally, the contributions of this study to the field of marine population genetics and future research needs are discussed.

\section{Major findings}

Microsatellite markers were specifically developed for P. novaezelandiae for this study (Chapter 2). Some loci showed significant deviation from Hardy-Weinberg equilibrium 
(HWE) and evidence of null alleles. Null alleles are frequently observed in bivalves (Hedgecock et al. 2004; Kenchington et al. 2006; Marín et al. 2012; Wei et al. 2013a) possibly causing departures from Hardy-Weinberg equilibrium (HWE) and the underestimation of heterozygote frequency. However, other factors such as spatial/temporal population structure could also contribute to deviation from HWE. In addition, results from this study are consistent with studies of other marine bivalves and simulations suggest that $F_{\text {ST }}$ values are unbiased when there is high gene flow, high effective population size $(\mathrm{Ne})$ and moderate frequencies of null alleles (Chapuis \& Estoup 2007), so null alleles are unlikely to significantly influence the conclusions of this study.

Low levels of genetic differentiation were detected for Pecten novaezelandiae across New Zealand (Chapter 3), which is expected given the large reproductive potential, the pelagic larval duration and the recent evolutionary history of this species. In general, population genetic structure was weak but there were significant differences between north, central and the southern New Zealand. The null hypothesis of panmixia was rejected since there was some evidence of genetic structure and an apparent hierarchical degree of differentiation from north to south.

The patterns of genetic variation of $P$. novaezelandiae across New Zealand were associated with a combination of environmental variables (Chapter 4). Although the geographic distance between populations was an important variable explaining the genetic variation, it appears that levels of genetic differentiation are not a simple function of distance. Evidence suggests that freshwater discharge and suspended particulate matter might be important factors contributing to the genetic structure of $P$. novaezelandiae in New Zealand.

Complex spatial and temporal patterns of genetic structure were observed for $P$. novaezelandiae in the Coromandel fishery region (Chapter 5). While low levels of genetic structure were detected, temporal instability patterns suggested a variable source of recruits. There was evidence of source-sink population dynamics, where populations in source habitats appeared to sustain populations both in source and sink habitats. Therefore, the null hypothesis of panmixia in the Coromandel fishery region was rejected. 


\section{Evolutionary consequences}

With a growing realisation that fisheries can induce evolutionary responses in the life history of harvested species (Kuparinen \& Merilä 2007), the importance of considering evolutionary processes in fisheries management and conservation is increasingly evident. Species are often distributed into spatially discrete 'populations' that form the basic units on which evolutionary forces, such as drift, gene flow, mutation and selection act over time. These forces determine the allelic composition of and variation between populations, and therefore different patterns of genetic variation can emerge within a species' range (Hedgecock et al. 2007). A full understanding of these evolutionary processes will allow fisheries management to adapt and prevent/reduce fisheries-induced impacts on productivity while maintaining the potential for evolutionary change in the future.

Complex historical and contemporary processes determine the population genetic structure of $P$. novaezelandiae. The patterns of genetic variation observed for $P$. novaezelandiae throughout New Zealand reflect a hierarchical degree of differentiation from north to south and also a latitudinal genetic diversity gradient. These patterns might be reflecting a recent colonisation event southwards and insufficient time to reach migrationdrift equilibrium. The low level of genetic differentiation observed between the Chatham Islands and mainland New Zealand suggests that larvae of $P$. novaezelandiae have a high dispersal potential, which might be preventing population subdivision. On the other hand, significant differences were observed between populations in the south of New Zealand, in habitats where other species also have shown significant genetic differentiation (e.g. Mladenov et al. 1997; reviewed in Gardner et al. 2010).

Population genetic studies are largely based on the use of neutral genetic markers, where gene flow and genetic drift are considered the main forces driving population genetic structure of organisms. Such neutral processes are likely to be shaping the genetic structure of $P$. novaezelandiae, with the south of New Zealand, for example, having highly variable oceanic currents and higher eddy diffusivity, which may be preventing larval exchange with other populations (Chiswell \& Rickard 2011) and therefore increasing levels of genetic differentiation. Also, although there is evidence that geographic distance between populations is an important variable explaining the genetic variation of $P$. novaezelandiae, it is not evident that the distance between the Chatham Islands and the mainland acts as an 
important barrier to larval dispersal. The subtropical convergence flowing eastward might be facilitating dispersal and gene flow from mainland New Zealand to the Chatham Islands and thus could explain the lower levels of differentiation.

Local adaptation has also been proposed as an indirect mechanism structuring neutral genetic variation within and among natural populations. For example, colonization processes can influence genetic variation of $P$. novaezelandiae. Rapid population growth and local adaptation of a new habitat can result in a successful monopolisation of resources, giving these resident individuals a strong priority effect (De Meester et al. 2002; Orsini et al. 2013). Genetic differentiation arising from founder events can last thousands of generations (Boileau et al. 1992). Therefore, in areas with high population densities, locally adapted individuals of $P$. novaezelandiae could be favoured over immigrants, maintaining population genetic subdivision even when levels of migration are high. Also, it has been shown for other scallop species that populations at higher latitudes spawn earlier in the season and over longer periods of time (Shumway \& Parsons 2006), which could also favour locally adapted individuals over immigrants coming from lower latitudes.

Freshwater discharge and suspended particulate matter were identified as important environmental factors shaping the genetic structure of $P$. novaezelandiae. As microsatellites are assumed to be neutral genetic markers (Freeland et al. 2011), these environmental variables can be influencing the genetic variation by neutral processes such as gene flow, genetic drift and mutation. However, it is debatable whether any locus is ever absolutely neutral (Liggins et al. 2013) because tests for selection generally have low power (Slatkin 1994, 1996) and it is unclear how many studies that used neutral genetic markers have been influenced by selection (Hudson \& Turelli 2003; Yang \& Nielsen 2008).

The association between genetic differentiation and the environmental factors 'freshwater discharge' and 'suspended particulate matter' has been shown for other marine species (Bricelj \& Malouf 1984; Bacon et al. 1998; Gardner \& Palmer 1998; Jørgensen et al. 2005; Hannan 2014). Exposure to suspended particulate matter has also been shown to affect both the feeding behaviour and growth rate of the scallop Pecten maximus (Szostek et al. 2013). Results from this study are in agreement with our knowledge about the biology and ecology of scallops regarding the low tolerance to variation in levels of suspended particulate matter and salinity (Shumway \& Parsons 2006). It is possible that exposure to higher levels of suspended particulate matter and/or freshwater discharge are placing strong 
selective pressure on $P$. novaezelandiae inhabiting such habitats, favouring locally adapted individuals, which might indirectly modify their genetic variation.

In addition, environmental features of more closed habitats such as harbours, gulfs and inlets are likely to influence larval dispersal processes and population dynamics. The Coromandel fishery area in north-eastern New Zealand encompasses the Hauraki Gulf, a semi-enclosed area and an open coastal environment, both characterised by a complex hydrodynamic environment. The physical oceanography and nutrient supply of both environments are highly influenced by the East Auckland Current (EAUC) flowing southeast (Zeldis 2004; Zeldis et al. 2004). This study suggests that patterns of dispersal of $P$. novaezelandiae in the region are characterized by a complex source-sink model of population dynamics. Also, the temporal decrease in genetic diversity observed can be the result of overfishing, stochastic oceanographic conditions or temporal variability in recruitment success. Genetic diversity is essential to maintain the evolutionary potential and adaptive ability of species (Allendorf et al. 2008). Populations with lower genetic diversity are less productive and resistant to disturbance or disease than populations with higher genetic diversity. If genetic diversity of $P$. novaezelandiae continues to decay it may lead to a long-term impact on the evolutionary potential.

Dispersal limitation, local adaptation and colonization history play an important role in shaping the genetic structure of species, both at neutral and non-neutral genetic variations (Orsini et al. 2013). It is likely that all these processes are driving the observed patterns of genetic structure in $P$. novaezelandiae and that complex relationships between these processes are placing strong selective pressure on scallops inhabiting specific habitats.

\section{Fisheries management implications}

Combining genetic markers with ecological information is a powerful approach to identify evolutionarily meaningful management units or stocks. This study was the first to investigate genetic variation of $P$. novaezelandiae at different geographic and temporal scales with the aim of representing the complexity of population dynamics. The findings of this study have important implications for the management of the scallop $P$. novaezelandiae throughout New Zealand and in particular, in the Coromandel scallop fishery. 


\section{Fisheries management in New Zealand}

The complexity of the New Zealand marine environment is favourable to produce a variety of patterns of genetic structure. Comparing connectivity patterns among species can help establish common biogeographic barriers and provide knowledge at the ecosystem level, both of which are required for management efforts. Levels of connectivity were high between populations of $P$. novaezelandiae, but there was an evident hierarchical degree of differentiation from north to south. Similar patterns of genetic structure have been observed for other species in New Zealand (Gardner et al. 2010; Westfall 2011; Ross et al. 2012), but in this study there was not evidence of a clear barrier to gene flow for $P$. novaezelandiae.

Compared to northern New Zealand, the southern region showed higher levels of differentiation as a result of lower genetic and demographic connectivity. Management of these southern stocks should be particularly cautious because they showed lower levels of genetic diversity and separate stocks may correspond to smaller geographic areas than the quota management areas currently accounted for by the quota management system. Populations within inlets and fiords are likely to experience lower connectivity to populations in the open marine environment, resulting in higher genetic differentiation. There is also the possibility that individuals within these areas such as Fiordland and Paterson Inlet on Stewart Island are locally adapted to specific environmental conditions. This would have serious implications for stock recovery if dependent on external sources of recruits that are adapted to open coast environment conditions.

Findings from this study that freshwater discharge and suspended particulate matter might be shaping the genetic structure of $P$. novaezelandiae have important management implications. Individuals can be adapted to the local environment and a shift in conditions such as, for example, variation in oceanographic cycles and high rainfall or an increase in sediment discharge in urbanised areas or from areas of heavy logging activity, can compromise the productivity of stocks. Terrestrial and marine ecosystems are intricately linked; however, current management practices rarely consider the effects of land-sea 
interactions and ignoring such interactions can result in failing to achieve management and conservation goals (Stoms et al. 2005).

Determining meaningful marine biogeographic patterns in New Zealand is an important step to develop effective planning units at a large spatial scale and appropriate scales and boundaries for management. In marine systems these biogeographic boundaries are intrinsically dynamic because of the complex environmental conditions and different life histories of species. The New Zealand marine environment is particularly complex with small currents and eddies, variable wind, waves and tides as well as coastal currents shaped by interactions with ocean floor topography (Ross et al. 2009). These characteristics represent a challenge to scientists, fisheries managers and conservationists, and have created difficulties in predicting the influence of the marine environment on population structure of species and in defining clear biogeographic provinces in the New Zealand marine environment (Shears et al. 2008).

As a result of higher genetic connectivity, populations in the north of New Zealand tend to show a lower degree of population differentiation compared to the southern region. However, unexpected small-scale patterns of differentiation can arise and may change over time. There was also evidence that self-recruitment may play an important role in maintaining productivity of stocks in the region. Understanding this complex dynamic at different geographic scales is essential for establishing effective management plans therefore, management implications of this study for the Coromandel scallop fishery are discussed below.

\section{The Coromandel scallop fishery (SCA CS)}

The Coromandel fishery area is characterized by a complex hydrodynamic environment. The East Auckland Current (EAUC) flowing southeast influences both the Hauraki Gulf and the open coast environment, creating complex eddies that can greatly influence population dynamics of Pecten novaezelandiae at small spatial scales (Chiswell \& Rickard 2011). This study detected a source-sink model of population dynamics, which has important implications for fisheries management of the New Zealand scallop in the Coromandel region. Gene flow estimates between locations need to be taken with caution because of the limitations of the assignment tests (Berry et al. 2004). However, the 
oceanographic model supported the results of assignment tests, which provides some confidence that dispersal levels in the Coromandel fishery are reasonably well represented.

Scallop populations that conform to a source-sink model may be best managed by precautionary management of the source populations. There was evidence that some of the sampled locations, which coincided with important fisheries such as 'Mercury' and 'Plenty', were sink populations. This means that adults of scallops inhabiting these sink habitats are less likely to contribute to the larval pool. On the other hand, some of the sampled locations that coincided with the very productive fishing areas 'Hauraki' and 'Barrier' appeared to sustain populations both in source and sink habitats, and therefore are likely to be source populations.

The complex dynamics of Pecten novaezelandiae in the Coromandel region is potentially influenced by a combination of oceanographic conditions such as the East Auckland Current (EAUC) that may be facilitating larval transport from 'Barrier' to southern populations and other environmental factors such as water depth in 'Hauraki', where individuals may spawn earlier in the season (Tunbridge 1968) and are more likely to occupy habitats at settlement locations early in the season. In addition, some populations such as 'Hauraki' may rely heavily on self-recruitment, in which case a localised precautionary management approach would be appropriate. The reliance on selfrecruitment poses a risk when stocks are heavily exploited and can become depleted, since recovery and rebuilding of biomass to sustainable levels may take a long time (Worm et al. 2009). This recovery can be even more problematic if recruits are not adapted to local environmental conditions such as water depth in 'Hauraki'. Given this, this population is probably more vulnerable to over-exploitation.

\section{Contribution to the field and future directions}

Findings of this study bring important contributions to fisheries management of Pecten novaezelandiae, significant knowledge about marine connectivity in New Zealand and important findings regarding the processes that can shape population genetic structure of species. New microsatellite markers were developed for $P$. novaezelandiae and these powerful molecular markers were used in this first study of population genetics of the endemic New Zealand scallop to address important ecological questions. The 
multidisciplinary approach used in this research highlights the importance of considering environmental factors in population genetic studies. In general, these findings can be applied to the management of $P$. novaezelandiae around New Zealand and in the Coromandel fishery area, and potentially to other bivalve species with similar life history characteristics and inhabiting similar habitats. Identifying common patterns among species and factors shaping those patters is crucial to our understanding of evolutionary and ecological processes as well as for management and conservation efforts. Therefore, the results of this study add to the increasing evidence that many marine species can show considerable genetic structure despite high dispersal potential, and can contributed to our understanding of evolutionary and population processes influencing marine connectivity and shaping population structure of marine species.

It has been recognised that a comprehensive understanding of population dynamics involves integration of methods that estimate dispersal over different spatial and temporal scales (Anderson et al. 2010). Results from this study highlight the importance of using different temporal and spatial scales in population genetic studies. Genetic differentiation can change across multiple temporal scales, which can influence interpretations about spatial patterns of differentiation (Toonen \& Grosberg 2011). Population genetic studies with samples obtained over multiple years can better elucidate about the complex processes producing temporal instability. However, there are several difficulties associated with sampling marine invertebrates over multiple temporal and spatial scales.

The use of high resolution physical modelling is a helpful approach that can effectively predict the patterns of connectivity (Werner et al.; Gallego et al. 2007), helping to answer several ecological questions. Physical models are frequently combined with detailed biological variables to provide realistic estimates of dispersal. Individual-Based Coupled Physical-Biological Models (ICPBMs), which incorporate biological characteristics of species such as larval behaviour and estimates of mortality are becoming increasingly popular (Miller 2007; Reidenbach et al. 2009; Bolle et al. 2009; Berry et al. 2012). One of the first studies integrating oceanographic and genetic models was developed by Galindo et al. (2006) to predict gene flow among locations of a reef-building coral across the Caribbean Sea. ICPBMs are evolving rapidly, becoming more spatially resolved and including more biological detail (Miller 2007). This is an increasingly important tool in advancing our understanding of larval dispersal and recruitment but it has yet to be widely 
applied in population genetic studies, particularly in New Zealand (but see Le Port et al. 2014). A particle tracking technique was used in this study as an exploratory analysis to estimate connectivity from specified source regions (Chapter 5). Unfortunately, a higher resolution model was not available for this study but a better understanding of processes shaping the population dynamics of $P$. novaezelandiae in the Coromandel fishery region and across New Zealand can be potentially achieved with an improved model.

An important finding of this research was the evidence of a source-sink model of population dynamics for P. novaezelandiae in the Coromandel fishery area. However, these results must be interpreted with caution since other sink/source populations may be present in the region but not sampled, and the assignment analyses have limitations given the low levels of genetic differentiation among populations (Berry et al. 2004). Applying a suitable hydrodynamic model for the entire Coromandel region, including the inner part of the Hauraki Gulf, would help elucidate the patterns of larval dispersal and connectivity between scallop's beds. In addition, the translocation of animals from sink to source populations can potentially be implemented as a complementary management tool for maintaining the genetic diversity of populations, which has decreased temporally (Gardner \& Van Putten 2008).

To fully understand evolutionary and population dynamic processes shaping patterns of population structure in the New Zealand marine environment, it is necessary to compare studies among multiple species. Meta-analyses can significantly improve our knowledge and resolve uncertainty about common patterns of genetic structure by combining the results from multiple studies. For example, Weersing \& Toonen (2009) used a metaanalysis approach to investigate the link between pelagic larval duration (PLD) and connectivity among populations, challenging the conventional view that PLD is a good predictor of the extent of gene flow in marine systems. Also, Toonen et al. (2011) conducted a study of 27 taxonomically and ecologically diverse species and showed the presence of four concordant barriers to dispersal within the Hawaiian Archipelago which were not detected in single-species studies. A meta-analysis of population genetic studies conducted for New Zealand marine species linking the patterns of genetic variation with environmental variation, would be the first step towards a better understanding of the processes shaping coastal population structure and therefore, defining meaningful boundaries at which to apply ecosystem-based fishery management (EBFM). 
In general, the typical fisheries management approach focuses on a single target species and often ignores interactions with other species and with the ecosystem. Prioritising the ecosystem rather than the target species is the main objective of ecosystem-based fishery management (EBFM; Slocombe 1993; Pikitch et al. 2004). A full understanding of ecosystem processes is essential for implementing EBFM; however, data are not always available when implementing management strategies. Because fisheries systems are complex, management should embrace uncertainty and be precautionary. Management should utilise an adaptive approach, continually testing how ecosystems respond to fisheries pressure and management strategies, which has been shown to improve our understanding of ecosystem dynamics (Hughes et al. 2007; Silva et al. 2013). Finally, intensive fishing can induce changes in life-history traits, behaviour, physiology, and morphology of species, which in turn can dramatically change the monetary value of resources. Therefore it is vital to assess the impacts of fisheries induced evolution (FIE) and assess the evolutionary consequences of fishing within an ecosystem-based management approach (Laugen et al. 2014).

This study provides important information about the processes that influence population dynamics of species. Further development should include improving models of larval dispersal and marine connectivity by expanding the use of multidisciplinary tools in areas such as physics, statistics, larval ecology and genetics (Selkoe et al. 2008; Cowen \& Sponaugle 2009). Successful management of $P$. novaezelandiae and other marine resources will require on-going monitoring and a good understanding of relevant ecological and evolutionary processes in the face of growing exploitation and changing environmental conditions. 


\section{References}

Abdelkrim J, Robertson B, Stanton J-A, Gemmell N (2009) Fast, cost-effective development of species-specific microsatellite markers by genomic sequencing. BioTechniques, 46, 185-92.

Aguirre JD, Marshall DJ (2012) Genetic diversity increases population productivity in a sessile marine invertebrate. Ecology, 93, 1134-1142.

Allendorf FW, Berry O, Ryman N (2014) So long to genetic diversity, and thanks for all the fish. Molecular Ecology, 23, 23-5.

Allendorf FW, England PR, Luikart G, Ritchie PA, Ryman N (2008) Genetic effects of harvest on wild animal populations. Trends in Ecology \& Evolution, 23, 327-337.

Anderson CD, Epperson BK, Fortin M-J, Holderegger R, James PMA, Rosenberg MS, Scribner KT, Spear S (2010) Considering spatial and temporal scale in landscapegenetic studies of gene flow. Molecular Ecology, 19, 3565-3575.

Antao T, Lopes A, Lopes RJ, Beja-Pereira A, Luikart G (2008) LOSITAN: a workbench to detect molecular adaptation based on a Fst-outlier method. BMC bioinformatics, 9 , 323.

Apte S, Gardner JPA (2001) Absence of population genetic differentiation in the New Zealand greenshell mussel Perna canaliculus (Gmelin 1791) as assessed by allozyme variation. Journal of Experimental Marine Biology and Ecology, 258, 173-194.

Apte S, Gardner JPA (2002) Population genetic subdivision in the New Zealand greenshell mussel (Perna canaliculus) inferred from single-strand conformation polymorphism analysis of mitochondrial DNA. Molecular Ecology, 11, 1617-28.

Arias A, Freire R, Méndez J, Insua A (2010) Isolation and characterization of microsatellite markers in the queen scallop Aequipecten opercularis and their application to a population genetic study. Aquatic Living Resources, 23, 199-207.

Avise JC (2004) Molecular Markers, Natural History and Evolution (Second Edition). Sunderland: Sinauer Associates, MA.

Ayers KL, Waters JM (2005) Marine biogeographic disjunction in central New Zealand. Marine Biology, 147, 1045-1052.

Bacon GS, Macdonald BA, Ward JE (1998) Physiological responses of infaunal (Mya arenaria) and epifaunal (Placopecten magellanicus) bivalves to variations in the concentration and quality of suspended particles I. Feeding activity and selection. Journal of Experimental Marine Biology and Ecology, 219, 105-125. 
Barber PH, Moosa MK, Palumbi SR (2002) Rapid recovery of genetic diversity of stomatopod populations on Krakatau: temporal and spatial scales of marine larval dispersal. Proceedings of The Royal Society Biological Sciences, 269, 1591-1597.

Barber PH, Palumbi SR, Erdmann M V, Moosa MK (2000) A marine Wallace's line? Science, 406, 692-693.

Barson NJ, Cable J, Van Oosterhout C (2009) Population genetic analysis of microsatellite variation of guppies (Poecilia reticulata) in Trinidad and Tobago: evidence for a dynamic source-sink metapopulation structure, founder events and population bottlenecks. Journal of Evolutionary Biology, 22, 485-97.

Beaumont AR, Barnes DA (1992) Aspects of veliger larval growth and byssus drifting of the spat of Pecten maximus and Aequipecten (Chlamys) opercularis. ICES Journal of Marine Science, 49, 417-423.

Berry O, England P, Fairclough D, Jackson G, Greenwood J (2012) Microsatellite DNA analysis and hydrodynamic modelling reveal the extent of larval transport and gene flow between management zones in an exploited marine fish (Glaucosoma hebraicum). Fisheries Oceanography, 21, 243-254.

Berry O, Tocher MD, Sarre SD (2004) Can assignment tests measure dispersal? Molecular Ecology, 13, 551-561.

Beu AG (2010) Marine Mollusca of oxygen isotope stages of the last 2 million years in New Zealand. Part 2. Biostratigraphically useful and new Pliocene to recent bivalves. Journal of the Royal Society of New Zealand, 36, 151-338.

Beu AG, Maxwell PA (1990) Cenozoic Mollusca of New Zealand. New Zealand Geological Survey, Lower Hutt, New Zealand.

Bohonak AJ (1999) Dispersal, gene flow, and population structure. The quarterly review of Biology, 74, 21-45.

Boileau MG, Hebert PDN, Schwartz SS (1992) Non-equilibrium gene frequency divergence: persistent founder effects in natural populations. Journal of Evolutionary Biology, 5, 25-39.

Bolle L, Dickey-Collas M, van Beek J, Erftemeijer P, Witte J, van der Veer H, Rijnsdorp A (2009) Variability in transport of fish eggs and larvae. III. Effects of hydrodynamics and larval behaviour on recruitment in plaice. Marine Ecology Progress Series, 390, $195-211$.

Bonin A, Nicole F, Pompanon F, Miaud C, Taberlet P (2007) Population adaptive index: a new method to help measure intraspecific genetic diversity and prioritize populations for conservation. Conservation biology: the journal of the Society for Conservation Biology, 21, 697-708. 
Boyer T, Levitus S, Garcia H, Locarnini R, Stephens C, Antonov J (2005) Objective analyses of annual, seasonal, and monthly temperature and salinity for the world on a 1/4degree Grid. International Journal of Climatology, 25, 931-945.

Bricelj VM, Krause MK (1992) Resource allocation and population genetics of the bay scallop, Argopecten irradians irradians: effects of age and allozyme heterozygosity on reproductive output. Marine Biology, 113, 253-261.

Bricelj VM, Malouf RE (1984) Influence of algal and suspended sediment concentrations on the feeding physiology of the hard clam Mercenaria mercenaria. Marine Biology, 165, 155-165.

Broquet T, Viard F, Yearsley JM (2013) Genetic drift and collective dispersal can result in chaotic genetic patchiness. Evolution, 67, 1660-1675.

Bull MF (1976) Aspects of the biology of the New Zealand scallop, Pecten novaezelandiae Reeve 1853, in the Marlborough Sounds. Victoria University of Wellington.

Cadrin SX, Friedland KD, Waldman JR (2005) Stock identification methods - Applications in fishery science. Elsevier Inc.

Carbines GD, Michael KP (2007) Status of the non-commercial scallop (Pecten novaezealandiae) fishery in Paterson Inlet, Stewart Island, and information needs for future management. New Zealand Fisheries Assessment Report 2007/4.

Carvalho GR (1998) Advances in Molecular Ecology (NATO Science Series A: Life Sciences). IOS Press Amsterdam, pp. 1-24.

Carvalho GR, Hauser L (1994) Molecular genetics and the stock concept in fisheries. Reviews in Fish Biology and Fisheries, 4, 326-350.

Chapuis M-P, Estoup A (2007) Microsatellite null alleles and estimation of population differentiation. Molecular Biology and Evolution, 24, 621-631.

Charrier G, Morvezen R, Calves I, Laroche J (2012) Development of new microsatellite markers derived from expressed sequence tags for the great scallop (Pecten maximus). Conservation Genetics Resources, 4, 931-934.

Chiswell S, Booth J (2008) Sources and sinks of larval settlement in Jasus edwardsii around New Zealand: Where do larvae come from and where do they go? Marine Ecology Progress Series, 354, 201-217.

Chiswell SM, Rickard GJ (2011) Larval connectivity of harbours via ocean currents: A New Zealand study. Continental Shelf Research, 31, 1057-1074.

Christie MR, Johnson DW, Stallings CD, Hixon MA (2010) Self-recruitment and sweepstakes reproduction amid extensive gene flow in a coral-reef fish. Molecular Ecology, 19, 1042-57. 
Clarke KR, Gorley RN (2006) PRIMER v6: User Manual/Tutorial. PRIMER-E, Plymouth.

Cole RG, Hull PJ, Healy TR (2000) Assemblage structure, spatial patterns, recruitment, and post-settlement mortality of subtidal bivalve molluscs in a large harbour in northeastern New Zealand. New Zealand Journal of Marine and Freshwater Research, 34, 317-329.

Conover DO, Clarke LM, Munch SB, Wagner GN (2006) Spatial and temporal scales of adaptive divergence in marine fishes and the implications for conservation. Journal of Fish Biology, 69, 21-47.

Constable HB (2014) Population structure, temporal stability and seascape genetics of two endemic New Zealand Pleuronectiformes, Rhombosolea plebeia (sand flounder) and $R$. leporina (yellowbelly flounder). PhD thesis. Victoria University of Wellington.

Cowen RK, Gawarkiewicz G, Pineda J, Thorrold SR, Werner FE (2007) Population Connectivity in Marine Systems - an overview. Oceanography, 20, 14-21.

Cowen RK, Sponaugle S (2009) Larval dispersal and marine population connectivity. Annual Review of Marine Science, 1, 443-66.

CSIRO (2014) “Connie 2.0”. CSIRO Connectivity Interface, http://www.csiro.au/connie2/.

D’Aloia CC, Bogdanowicz SM, Harrison RG, Buston PM (2014) Seascape continuity plays an important role in determining patterns of spatial genetic structure in a coral reef fish. Molecular Ecology, 23, 2902-2913.

D'Sa EJ, Miller RL, McKee BA (2007) Suspended particulate matter dynamics in coastal waters from ocean color: Application to the northern Gulf of Mexico. Geophysical Research Letters, 34, 1-6.

Dawson MN, Hays CG, Grosberg RK, Raimondi PT (2014) Dispersal potential and population genetic structure in the marine intertidal of the eastern North Pacific. Ecological Monographs, 84, 435-456.

Drake JM, Griffen BD (2013) Experimental demonstration of accelerated extinction in source-sink metapopulations. Ecology and Evolution, 3, 3369-3378.

Duchesne P, Turgeon J (2012) FLOCK provides reliable solutions to the "number of populations" problem. The Journal of Heredity, 103, 734-743.

Earl DA, VonHoldt BM (2012) STRUCTURE HARVESTER: a website and program for visualizing STRUCTURE output and implementing the Evanno method. Conservation Genetics Resources, 4, 359-361.

Eckert CG, Samis KE, Lougheed SC (2008) Genetic variation across species' geographical ranges: the central-marginal hypothesis and beyond. Molecular Ecology, 17, 11701188 . 
Evanno G, Regnaut S, Goudet J (2005) Detecting the number of clusters of individuals using the software STRUCTURE: a simulation study. Molecular Ecology, 14, 26112620.

Excoffier L, Lischer HEL (2010) Arlequin suite ver 3.5: a new series of programs to perform population genetics analyses under Linux and Windows. Molecular Ecology Resources, 10, 564-567.

Excoffier L, Smouse PE, Quattro JM (1992) Analysis of molecular variance inferred from metric distances among DNA haplotypes: application to human mitochondrial DNA restriction data. Genetics, 131, 479-91.

Fleming CA (1979) The geological history of NZ and its life. University of Auckland Bindery.

Flowers JM, Schroeter SC, Burton RS (2002) The recruitment sweepstakes has many winners: genetic evidence from the sea urchin Strongylocentrotus purpuratus. Evolution, 56, 1445-1453.

Frantz AC, Cellina S, Krier A, Schley L, Burke T (2009) Using spatial Bayesian methods to determine the genetic structure of a continuously distributed population: clusters or isolation by distance? Journal of Applied Ecology, 46, 493-505.

Freeland JR (2005) Molecular Ecology. The Open University, West Sussex, England.

Freeland JR, Petersen SD, Kirk H (2011) Molecular Ecology. Wiley-Blackwell, West Sussex, England.

Gaggiotti OE, Bekkevold D, Jørgensen HBH, Foll M, Carvalho GR, Andre C, Ruzzante DE (2009) Disentangling the effects of evolutionary, demographic, and environmental factors influencing genetic structure of natural populations: Atlantic herring as a case study. Evolution, 63, 2939-51.

Galindo HM, Olson DB, Palumbi SR (2006) Seascape genetics: a coupled oceanographicgenetic model predicts population structure of Caribbean corals. Current Biology, 16, 1622-6.

Galindo HM, Pfeiffer-Herbert AS, McManus MA, Chao Y, Chai F, Palumbi SR (2010) Seascape genetics along a steep cline: using genetic patterns to test predictions of marine larval dispersal. Molecular Ecology, 19, 3692-707.

Gallego A, North EW, Petitgas P (2007) Introduction: status and future of modelling physical-biological interactions during the early life of fishes. Marine Ecology Progress Series, 347, 121-126.

Gao X, Starmer JD (2008) AWclust: point-and-click software for non-parametric population structure analysis. BMC Bioinformatics, 9, 77. 
Gardner JPA, Bell JJ, Constable HB, Hannan D, Ritchie PA, Zuccarello GC (2010) Multispecies coastal marine connectivity: a literature review with recommendations for further research. Wellington. New Zealand Aquatic Environment and Biodiversity Report No. 58.

Gardner JPA, Palmer NL (1998) Size-dependent, spatial and temporal genetic variation at a leucine aminopeptidase (LAP) locus among blue mussel (Mytilus galloprovincialis) populations along a salinity gradient. Marine Biology, 132, 275-281.

Gardner C, Van Putten EI (2008) Biological Modeling of Translocation as a Management Tool for a Rock Lobster Fishery. Reviews in Fisheries Science, 16, 81-90.

Goldstien SJ, Schiel DR, Gemmell NJ (2006) Comparative phylogeography of coastal limpets across a marine disjunction in New Zealand. Molecular Ecology, 15, 3259-68.

González-Wangüemert M, Cánovas F, Pérez-Ruzafa A, Marcos C, Alexandrino P (2010) Connectivity patterns inferred from the genetic structure of white seabream (Diplodus sargus L.). Journal of Experimental Marine Biology and Ecology, 383, 23-31.

Gordon DP, Beaumont J, MacDiarmid A, Robertson DA, Ahyong ST (2010) Marine biodiversity of Aotearoa New Zealand. PloS one, 5, e10905.

Goudet J, Raymond M, de Meeüs T, Rousset F (1996) Testing differentiation in diploid populations. Genetics, 144, 1933-40.

Gruber MAM, Hoffmann BD, Ritchie PA, Lester PJ (2013) The conundrum of the yellow crazy ant (Anoplolepis gracilipes) reproductive mode: no evidence for dependent lineage genetic caste determination. Insectes Sociaux, 60, 135-145.

Guichoux E, Lagache L, Wagner S, Chaumeil P, Léger P, Lepais O, Lepoittevin C, Malausa T, Revardel E, Salin F, Petit RJ (2011) Current trends in microsatellite genotyping. Molecular Ecology Resources, 11, 591-611.

Guillot G (2008) Inference of structure in subdivided populations at low levels of genetic differentiation - the correlated allele frequencies model revisited. Bioinformatics, 24, 2222-2228.

Guillot G, Renaud S, Ledevin R, Michaux J, Claude J (2012) A unifying model for the analysis of phenotypic, genetic, and geographic data. Systematic Biology, 61, 897-911.

Hall SJ (1994) Physical disturbance and marine benthic communities: life in unconsolidated sediments. Oceanography and Marine Biology - An Annual Review, 32, 179-239.

Hannan DA (2014) Population genetics and connectivity in Paphies subtriangulata and Paphies australis (Bivalvia: Mesodesmatidae). $\mathrm{PhD}$ thesis. Victoria University of Wellington. 
Hasselman DJ, Ricard D, Bentzen P (2013) Genetic diversity and differentiation in a wide ranging anadromous fish, American shad (Alosa sapidissima), is correlated with latitude. Molecular Ecology, 1558-1573.

Hauser L, Adcock GJ, Smith PJ, Ramiréz JHB, Carvalho GR (2002) Loss of microsatellite diversity and low effective population size in an overexploited population of New Zealand snapper (Pagrus auratus). Proceedings of the National Academy of Sciences of the United States of America, 99, 11742-7.

Hauser L, Carvalho GR (2008) Paradigm shifts in marine fisheries genetics: ugly hypotheses slain by beautiful facts. Fish and Fisheries, 9, 333-362.

Hedgecock D (1982) Genetic consequences of larval retention: theoretical and methodological aspects. In: Estuarine comparisons (ed Kennedy V), pp. 553-568. Academic Press, New York.

Hedgecock D (1986) Is gene flow from pelagic larval dispersal important in the adaptation and evolution of marine invertebrates? Bulletin of Marine Science, 39, 550-564.

Hedgecock D (1994) Does variance in reproductive success limit effective population size of marine organisms? In: Genetics and evolution of aquatic organisms. (ed Beaumont A), pp. 122-134. Chapman and Hall, London.

Hedgecock D, Barber PH, Edmands S (2007) Genetic Approaches to Measuring Connectivity. Oceanography, 20, 70-79.

Hedgecock D, Li G, Hubert S, Bucklin K, Ribes V (2004) Widespread null alleles and poor cross-species amplification of microsatellite DNA loci cloned from the Pacific oyster, Crassostrea gigas. Journal of Shellfish Research, 23, 379-385.

Hedgecock D, Pudovkin AI (2011) Sweepstakes Reproductive Success in Highly Fecund Marine Fish and Shellfish: A Review and Commentary. Bulletin of Marine Science, 87, 971-1002.

Heipel DA, Bishop JDD, Brand AR, Thorpe JP (1998) Population genetic differentiation of the great scallop Pecten maximus in western Britain investigated by randomly amplified polymorphic DNA. Marine Ecology Progress Series, 162, 163-171.

Hellberg ME, Burton RS, Neigel JE, Palumbi SR (2002) Genetic assessment of connectivity among marine populations. Bulletin of Marine Science, 70, 273-290.

Hemond EM, Wilbur AE (2011) Microsatellite loci indicate population structure and selection between Atlantic and Gulf of Mexico populations of the bay scallop Argopecten irradians. Marine Ecology Progress Series, 423, 131-142.

Hewitt GM (1996) Some genetic consequences of ice ages, and their role in divergence and speciation. Biological Journal of the Linnean Society, 58, 247-276. 
Hilbish TJ (1985) Demographic and temporal structure of an allele frequency cline in the mussel Mytilus edulis. Marine Biology, 87, 163-171.

Hogan J, Thiessen R, Heath D (2010) Variability in connectivity indicated by chaotic genetic patchiness within and among populations of a marine fish. Marine Ecology Progress Series, 417, 263-275.

Holleley CE, Geerts PG (2009) Multiplex Manager 1.0: a cross-platform computer program that plans and optimizes multiplex PCR. BioTechniques, 46, 511-7.

Hudson RR, Turelli M (2003) Stochasticity overrules the "three-times rule"": genetic drift, genetic draft and coalescence times for nuclear loci versus mitochondrial DNA. Evolution, 57, 182-190.

Hughes TP, Gunderson LH, Folke C, Baird AH, Bellwood D, Berkes F, Crona B, Helfgott A, Leslie H, Norberg J, Nystro M, Scheffer M, Schuttenberg H, Steneck RS, Olsson P, Henrik O, Troell M, Walker B, Wilson J et al. (2007) Adaptive Management of the Great Barrier Reef and the Grand Canyon World Heritage Areas. Ambio, 36, 586-592.

Johannesson K, André C (2006) Life on the margin: genetic isolation and diversity loss in a peripheral marine ecosystem, the Baltic Sea. Molecular Ecology, 15, 2013-29.

Johannesson K, Johannesson B (1989) Differences in allele frequencies of Aat between high- and mid-rocky shore populations of Littorina saxatilis (Olivi) suggest selection in this enzyme locus. Genetical Research, 54, 7-12.

Johannesson K, Johannesson B, Lundgren U (1995) Strong natural selection causes microscale allozyme variation in a marine snail. Proceedings of the National Academy of Sciences of the United States of America, 92, 2602-2606.

Johnson MS, Black R (1982) Chaotic Genetic Patchiness in an Intertidal Limpet, Siphonaria sp. Marine Biology, 70, 157-164.

Jong NE de (2013) Reproduction and larval development of the New Zealand scallop, Pecten novaezelandiae. MSc Thesis, Auckland University of Technology.

Jørgensen HBH, Hansen MM, Bekkevold D, Ruzzante DE, Loeschcke V (2005) Marine landscapes and population genetic structure of herring (Clupea harengus L.) in the Baltic Sea. Molecular Ecology, 14, 3219-34.

Jost L (2008) GST and its relatives do not measure differentiation. Molecular Ecology, 17, 4015-4026.

Kaartvedt S, Aksnes DL, Egge JK (1987) Effect of light on the vertical distribution of Pecten maximus larvae. Marine Ecology Progress Series, 40, 195-197.

Kalinowski ST (2005) Hp-Rare 1.0: a Computer Program for Performing Rarefaction on Measures of Allelic Richness. Molecular Ecology Notes, 5, 187-189. 
Kenchington EL, Patwary MU, Zouros E, Bird CJ (2006) Genetic differentiation in relation to marine landscape in a broadcast-spawning bivalve mollusc (Placopecten magellanicus). Molecular Ecology, 15, 1781-96.

Kesäniemi JE, Mustonen M, Boström C, Hansen BW, Knott KE (2014) Temporal genetic structure in a poecilogonous polychaete: the interplay of developmental mode and environmental stochasticity. BMC evolutionary biology, 14, 12.

Kovach AI, Breton TS, Berlinsky DL, Maceda L, Wirgin I (2010) Fine-scale spatial and temporal genetic structure of Atlantic cod off the Atlantic coast of the USA. Marine Ecology Progress Series, 410, 177-195.

Kuparinen A, Merilä J (2007) Detecting and managing fisheries-induced evolution. Trends in Ecology \& Evolution, 22, 652-659.

Larkin PA (1972) The Stock Concept in Pacific Salmon. In: stock identification workshop (eds Simon RC, Larkin PA), p. 231. University of British Columbia, Seattle, Washington.

Larson RJ, Julian RM (1999) Spatial and temporal genetic patchiness in marine populations and their implications for fisheries management. CalCOFl, 40, 94-99.

Laugen AT, Engelhard GH, Whitlock R, Arlinghaus R, Dankel DJ, Dunlop ES, Eikeset AM, Enberg K, Jørgensen C, Matsumura S, Nusslé S, Urbach D, Baulier L, Boukal DS, Ernande B, Johnston FD, Mollet F, Pardoe H, Therkildsen NO et al. (2014) Evolutionary impact assessment: accounting for evolutionary consequences of fishing in an ecosystem approach to fisheries management. Fish and Fisheries, 15, 65-96.

Leathwick JR, Rowden A, Nodder S, Gorman R, Bardsley S, Pinkerton MH, Baird SJ, Hadfield M, Currie K, Goh A (2012) A Benthic-optimised Marine Environment Classification (BOMEC) for New Zealand waters. New Zealand Aquatic Environment and Biodiversity Report No. 88.

Levin LA (2006) Recent progress in understanding larval dispersal: new directions and digressions. Integrative and comparative biology, 46, 282-97.

Lewis KB, Carter L, Davey FJ (1994) The opening of Cook Strait: Interglacial tidal scour and aligning basins at a subduction to transform plate edge. Marine Geology, 116, 293-312.

Lewis RI, Thorpe JP (1994) Temporal stability of gene frequencies within genetically heterogeneous populations of the queen scallop Aequipecten (Chlamys) opercularis. Marine Biology, 121, 117-126.

Li WH (1997) Molecular Evolution. Sinauer Associates, Sunderland, Massachusetts. 
Liggins L, Treml EA, Riginos C (2013) Taking the Plunge: An Introduction to Undertaking Seascape Genetic Studies and using Biophysical Models. Geography Compass, 7, 173-196.

Lipcius RN, Stockhausen WT, Eggleston DB, Marshall Jr LS, Hickey B (1997) Hydrodynamic decoupling of recruitment, habitat quality and adult abundance in the Caribbean spiny lobster: source-sink dynamics? Marine Freshwater Research, 48, 807-815.

Ma H, Yu Z (2009) Isolation and characterization of twenty-three microsatellite loci in the noble scallop, Chlamys nobilis. Conservation Genetics Resources, 1, 131-134.

Macleod JAA, Thorpe JP, Duggan NA (1985) A biochemical genetic study of population structure in queen scallop (Chlamys opercularis) stocks in the Northern Irish Sea. Marine Biology, 87, 77-82.

Manel S, Schwartz MK, Luikart G, Taberlet P (2003) Landscape genetics: combining landscape ecology and population genetics. Trends in Ecology \& Evolution, 18, 189197.

Marín A, Fujimoto T, Arai K (2012) Isolation and characterization of 12 microsatellite loci in the Peruvian scallop Argopecten purpuratus and cross-species amplification in other scallop species (family Pectinidae). Conservation Genetics Resources, 4, 179-182.

McFarlane GA, Wydoski RS, Prince EO (1990) A historical review of the development of external tags and marks. In: Proceedings of the International Tagging Symposium., Symp.7, pp. 9-29.

McLeod RJ, Wing SR (2008) Influence of an altered salinity regime on the population structure of two infaunal bivalve species. Estuarine, Coastal and Shelf Science, 78, $529-540$.

De Meester L, Gómez A, Okamura B, Schwenk K (2002) The Monopolization Hypothesis and the dispersal-gene flow paradox in aquatic organisms. Acta Oecologica, 23, 121135 .

Meglécz E, Costedoat C, Dubut V, Gilles A, Malausa T, Pech N, Martin J-F (2010) QDD: a user-friendly program to select microsatellite markers and design primers from large sequencing projects. Bioinformatics, 26, 403-4.

Meirmans PG (2006) Using the Amova framework to estimate a standardized genetic differentiation measure. Evolution, 60, 2399-2402.

Meirmans PG, Van Tienderen PH (2004) Genotype and Genodive: Two Programs for the Analysis of Genetic Diversity of Asexual Organisms. Molecular Ecology Notes, 4, 792-794. 
Miller TJ (2007) Contribution of individual-based coupled physical-biological models to understanding recruitment in marine fish populations. Marine Ecology Progress Series, 347, 127-138.

Ministry of Primary Industries (2012) The Status of New Zealand's Fisheries 2012. Wellington, New Zealand.

Ministry of Primary Industries (2013a) Fisheries Assessment Plenary November 2013: Stock Assessments and Yield Estimates - Scallops Coromandel (SCA CS). Wellington, New zealand.

Ministry of Primary Industries (2013b) The Status of New Zealand's Fisheries 2013. Wellington, New Zealand.

Ministry of Primary Industries (2014) Te Whaka $\bar{a}$ Te Wera/Paterson Inlet Mataitai Reserve Fishing Bylaws and Recreational Fishing Rules. Invercargill, New Zealand.

Mladenov P V., Allibone RM, Wallis GP (1997) Genetic differentiation in the New Zealand sea urchin Evechinus chloroticus (Echinodermata: Echinoidea). New Zealand Journal of Marine and Freshwater Research, 31, 261-269.

Morrison MA (1999) Population dynamics of the scallop Pecten novaezelandiae in the Hauraki Gulf. University of Auckland.

Nagashima K, Sato M, Kawamata K, Nakamura A, Ohta T (2005) Genetic structure of Japanese scallop population in Hokkaido, analyzed by mitochondrial haplotype distribution. Marine Biotechnology, 7, 1-10.

Nanninga GB, Saenz-Agudelo P, Manica A, Berumen ML (2014) Environmental gradients predict the genetic population structure of a coral reef fish in the Red Sea. Molecular Ecology, 23, 591-602.

NASA (2014) Ocean colour satellite data used courtesy of NASA Goddard Space Flight Center (MODIS project). Products generated by Matt Pinkerton, NIWA, Wellington.

Navarro E, Iglesias JIP (1993) Infaunal filter-feeding bivalves and the physiological response to short-term fluctuations in food availability and composition. In: Bivalve Filter Feeders in Estuarine and Coastal Ecosystem Processes (ed Dame RF), pp. 2556. NATO ASI Series, Vol. G33, Springer-Verlag, Berlin.

Neethling M, Matthee CA, Bowie RCK, von der Heyden S (2008) Evidence for panmixia despite barriers to gene flow in the southern African endemic, Caffrogobius caffer (Teleostei: Gobiidae). BMC Evolutionary Biology, 8, 325.

New Zealand Ministry for the Environment (2005) The New Zealand Marine Environment Classification. Wellington, $80 \mathrm{pp}$. 
Van Oosterhout C, Hutchinson WF, Wills DPM, Shipley P (2004) Micro-Checker: Software for Identifying and Correcting Genotyping Errors in Microsatellite Data. Molecular Ecology Notes, 4, 535-538.

Orsini L, Vanoverbeke J, Swillen I, Mergeay J, De Meester L (2013) Drivers of population genetic differentiation in the wild: isolation by dispersal limitation, isolation by adaptation and isolation by colonization. Molecular Ecology, 22, 5983-5999.

Owen EF, Rawson PD (2013) Small-scale spatial and temporal genetic structure of the Atlantic sea scallop (Placopecten magellanicus) in the inshore Gulf of Maine revealed using AFLPs. Marine Biology, 160, 3015-3025.

Paetkau DP, Slade R, Burden M, Estoup A (2004) Genetic assignment methods for the direct, real-time estimation of migration rate: a simulation-based exploration of accuracy and power. Molecular Ecology, 13, 55-65.

Pamilo P (1990) Comparison of Relatedness Estimators. Evolution, 44, 1378-1382.

Pande A, MacDiarmid AB, Smith PJ, Davidson RJ, Cole RG, Freeman D, Kelly S, Gardner JPA (2008) Marine reserves increase the abundance and size of blue cod and rock lobster. Marine Ecology Progress Series, 366, 147-158.

Park LK, Moran P (1994) Developments in molecular genetic techniques in fisheries. Reviews in Fish Biology and Fisheries, 4, 272-299.

Peakall R, Smouse PE (2006) GENALEX 6: genetic analysis in Excel. Population genetic software for teaching and research. Molecular Ecology Notes, 6, 288-295.

Peakall R, Smouse PE (2012) GenAlEx 6.5: genetic analysis in Excel. Population genetic software for teaching and research-an update. Bioinformatics, 28, 2537-9.

Pennec M Le, Paugam A, Pennec GL (2003) The pelagic life of the pectinid Pecten maximus - a review. ICES Journal of Marine Science, 60, 211-223.

Perrier C, Guyomard R, Bagliniere J-L, Nikolic N, Evanno G (2013) Changes in the genetic structure of Atlantic salmon populations over four decades reveal substantial impacts of stocking and potential resiliency. Ecology and Evolution, 3, 2334-2349.

Perrin C, Wing SR, Roy MS (2004) Effects of hydrographic barriers on population genetic structure of the sea star Coscinasterias muricata (Echinodermata, Asteroidea) in the New Zealand fiords. Molecular Ecology, 13, 2183-2195.

Petersen JL, Ibarra AM, May B (2010) Nuclear and mtDNA lineage diversity in wild and cultured Pacific lion-paw scallop, Nodipecten subnodosus (Baja California Peninsula, Mexico). Marine Biology, 157, 2751-2767.

Pikitch EK, Santora C, Babcock EA, Bakun A, Bonfil R, Conover DO, Dayton P, Doukakis P, Fluharty D, Heneman B, Houde ED, Link J, Livingston PA, Mangel M, McAllister 
MK, Pope J, Sainsbury KJ (2004) Ecosystem-based fishery management. Science, 305, 346-347.

Pilgrim BL, Perry RC, Keefe DG, Perry E a., Dawn Marshall H (2012) Microsatellite variation and genetic structure of brook trout (Salvelinus fontinalis) populations in Labrador and neighboring Atlantic Canada: evidence for ongoing gene flow and dual routes of post-Wisconsinan colonization. Ecology and Evolution, 2, 885-898.

Pineda J, Hare J, Sponaugle S (2007) Larval Transport and Dispersal in the Coastal Ocean and Consequences for Population Connectivity. Oceanography, 20, 22-39.

Pinkerton MH, Richardson KR (2005) Case 2 climatology of New Zealand: final report. NIWA Client Report WLG2005-49.

Pinsky ML, Palumbi SR (2014) Meta-analysis reveals lower genetic diversity in overfished populations. Molecular Ecology, 23, 29-39.

Piry S, Alapetite A, Cornuet J-M, Paetkau D, Baudouin L, Estoup A (2004) GENECLASS2: a software for genetic assignment and first-generation migrant detection. Journal of Heredity, 95, 536-539.

Pitcher TJ, Watson R, Forrest R, Valtysson HP, Guénette S (2002) Estimating illegal and unreported catches from marine ecosystems: a basis for change. Fish and Fisheries, $\mathbf{3}$, $317-339$.

Le Port A, Montgomery JC, Croucher AE (2014) Biophysical modelling of snapper Pagrus auratus larval dispersal from a temperate MPA. Marine Ecology Progress Series, 515, 203-215.

Pringle JM, Wares JP (2007) Going against the flow: maintenance of alongshore variation in allele frequency in a coastal ocean. Marine Ecology Progress Series, 335, 69-84.

Pritchard JK, Stephens M, Donnelly P (2000) Inference of population structure using multilocus genotype data. Genetics, 155, 945-59.

Pujolar JM, Maes GE, Volckaert FAM (2006) Genetic patchiness among recruits in the European eel Anguilla anguilla. Marine Ecology Progress Series, 307, 209-217.

Pulliam HR (1988) Sources, Sinks, and Population Regulation. The American Naturalist, 132, 652-661.

Pusack TJ, Christie MR, Johnson DW, Stallings CD, Hixon MA (2014) Spatial and temporal patterns of larval dispersal in a coral-reef fish metapopulation: evidence of variable reproductive success. Molecular Ecology, 23, 3396-3408.

Puslednik L, Serb JM (2008) Molecular phylogenetics of the Pectinidae (Mollusca: Bivalvia) and effect of increased taxon sampling and outgroup selection on tree topology. Molecular Phylogenetics and Evolution, 48, 1178-88. 
QGIS Development Team (2014) QGIS Geographic Information System. Open Source Geospatial Foundation Project. http://qgis.osgeo.org.

R Core Team (2014) R: A language and environment for statistical computing.

Rand DM, Spaeth PS, Sackton TB, Schmidt PS (2002) Ecological Genetics of Mpi and Gpi Polymorphisms in the Acorn Barnacle and the Spatial Scale of Neutral and Nonneutral Variation. Integrative and Comparative Biology, 42, 825-836.

Rassweiler A, Costello C, Siegel DA (2012) Marine protected areas and the value of spatially optimized fishery management. Proceedings of the National Academy of Sciences of the United States of America, 109, 11884-9.

Reidenbach MA, Koseff JR, Koehl MAR (2009) Hydrodynamic forces on larvae affect their settlement on coral reefs in turbulent, wave-driven flow. Limnology and Oceanography, 54, 318-330.

Reiss H, Hoarau G, Dickey-Collas M, Wolff WJ (2009) Genetic population structure of marine fish: mismatch between biological and fisheries management units. Fish and Fisheries, 10, 361-395.

Reisser CMO, Bell JJ, Gardner JPA (2014) Correlation between pelagic larval duration and realised dispersal: long-distance genetic connectivity between northern New Zealand and the Kermadec Islands archipelago. Marine Biology, 161, 297-312.

Reusch TBH, Bolte S, Sparwel M, Moss AG, Javidpour J (2010) Microsatellites reveal origin and genetic diversity of Eurasian invasions by one of the world's most notorious marine invader, Mnemiopsis leidyi (Ctenophora). Molecular Ecology, 19, 2690-9.

Riginos C, Liggins L (2013) Seascape Genetics: Populations, Individuals, and Genes Marooned and Adrift. Geography Compass, 7, 197-216.

Rios C, Sanz S, Saavedra C, Pena JB (2002) Allozyme variation in populations of scallops, Pecten jacobaeus (L.) and P. maximus (L.) (Bivalvia: Pectinidae), across the AlmeriaOran front. Journal of Experimental Marine Biology and Ecology, 267, 223-244.

Rioux Paquette S (2011) PopGenKit: useful function for (batch) file conversion and data resampling in microsatellite datasets. $\mathrm{R}$ package.

Robainas-Barcia A, Blanco G, Sánchez JA, Monnerot M, Solignac M, García-Machado E (2008) Spatiotemporal genetic differentiation of Cuban natural populations of the pink shrimp Farfantepenaeus notialis. Genetica, 133, 283-294.

Ross PM, Hogg ID, Pilditch CA, Lundquist CJ (2009) Phylogeography of New Zealand' s coastal benthos. New Zealand Journal of Marine and Freshwater Research, 43, 10091027. 
Ross PM, Hogg ID, Pilditch CA, Lundquist CJ, Wilkins RJ (2012) Population Genetic Structure of the New Zealand Estuarine Clam Austrovenus stutchburyi (Bivalvia: Veneridae) Reveals Population Subdivision and Partial Congruence with Biogeographic Boundaries. Estuaries and Coasts, 35, 143-154.

Rousset F (2008) genepop'007: a complete re-implementation of the genepop software for Windows and Linux. Molecular Ecology Resources, 8, 103-6.

Ryman N, Palm S (2006) POWSIM: a computer program for assessing statistical power when testing for genetic differentiation. Molecular Ecology, 6, 600-602.

Saavedra C, Peña JB (2006) Phylogenetics of American scallops (Bivalvia: Pectinidae) based on partial 16S and 12S ribosomal RNA gene sequences. Marine Biology, 150, $111-119$.

Saenz-Agudelo P, Jones GP, Thorrold SR, Planes S (2012) Patterns and persistence of larval retention and connectivity in a marine fish metapopulation. Molecular Ecology, 21, 4695-705.

Sagarin RD, Gaines SD (2002) The "abundant centre" distribution: to what extent is it a biogeographical rule? Ecology Letters, 5, 137-147.

Saillant EA, Renshaw MA, Cummings NJ, Gold JR (2012) Conservation genetics and management of yellowtail snapper, Ocyurus chrysurus, in the US Caribbean and South Florida. Fisheries Management and Ecology, 19, 301-312.

Saitou N, Nei M (1987) The neighbor-joining method: a new method for reconstructing phylogenetic trees. Molecular Biology and Evolution, 4, 406-425.

Salvi D, Bellavia G, Cervelli M, Mariottini P (2010) The analysis of rRNA sequencestructure in phylogenetics: An application to the family Pectinidae (Mollusca: Bivalvia). Molecular Phylogenetics and Evolution, 56, 1059-67.

Sato M, Kawamata K, Zaslavskaya N, Nakamura A, Ohta T, Nishikiori T, Brykov V, Nagashima K (2005) Development of microsatellite markers for Japanese scallop (Mizuhopecten yessoensis) and their application to a population genetic study. Marine Biotechnology, 7, 713-28.

Schuelke M (2000) An economic method for the fluorescent labeling of PCR fragments. Nature Biotechnology, 18, 233-234.

Schunter C, Carreras-Carbonell J, Macpherson E, Tintoré J, Vidal-Vijande E, Pascual A, Guidetti P, Pascual M (2011) Matching genetics with oceanography: directional gene flow in a Mediterranean fish species. Molecular Ecology, 20, 5167-5181.

Selkoe KA, Henzler CM, Gaines SD (2008) Seascape genetics and the spatial ecology of marine populations. Fish and Fisheries, 9, 363-377. 
Selkoe KA, Toonen RJ (2006) Microsatellites for ecologists: a practical guide to using and evaluating microsatellite markers. Ecology Letters, 9, 615-29.

Selkoe KA, Watson JR, White C, Horin T Ben, Iacchei M, Mitarai S, Siegel DA, Gaines SD, Toonen RJ (2010) Taking the chaos out of genetic patchiness: seascape genetics reveals ecological and oceanographic drivers of genetic patterns in three temperate reef species. Molecular Ecology, 19, 3708-3726.

Shanks AL (2009) Pelagic larval duration and dispersal distance revisited. The Biological Bulletin, 216, 373-85.

Shears NT, Smith F, Babcock RC, Duffy CAJ, Villouta E (2008) Evaluation of Biogeographic Classification Schemes for Conservation Planning: Application to New Zealand's Coastal Marine Environment. Conservation Biology, 22, 467-481.

Shirk RY, Hamrick JL, Zhang C, Qiang S (2014) Patterns of genetic diversity reveal multiple introductions and recurrent founder effects during range expansion in invasive populations of Geranium carolinianum (Geraniaceae). Heredity, 112, 497507.

Shumway SE, Parsons GJ (2006) Scallops: Biology, Ecology and Aquaculture (SE Shumway, GJ Parsons, Eds,). Elsevier B.V.

Siegel DA, Mitarai S, Costello CJ, Gaines SD, Kendall BE, Warner RR, Winters KB (2008) The stochastic nature of larval connectivity among nearshore marine populations. Proceedings of the National Academy of Sciences of the United States of America, 105, 8974-8979.

Silva CNS, Broadhurst MK, Medeiros RP, Dias JH (2013) Resolving environmental issues in the southern Brazilian artisanal penaeid-trawl fishery through adaptive comanagement. Marine Policy, 42, 133-141.

Silva CNS, Gardner JPA (2014) Development and characterisation of 12 microsatellite markers for the New Zealand endemic scallop Pecten novaezelandiae. Conservation Genetics Resources, 6, 327-328.

Slatkin M (1994) An exact test for neutrality based on the Ewens sampling distribution. Genetical Research, 64, 71-74.

Slatkin M (1995) A measure of population subdivision based on microsatellite allele frequencies. Genetics, 139, 457-62.

Slatkin M (1996) A correction to the exact test based on the Ewens sampling distribution. Genetical Research, 68, 259-260.

Slocombe DS (1993) Implementing ecosystem-based Management. BioScience, 43, 612622. 
Snelder T, Leathwick J, Day K, Weatherhead M, Fenwick G, Francis M, Gorman R, Grieve J, Hadfield M, Hewitt J, Hume T, Richardson K, Rowden A, Uddstrom M, Wild M, Zeldis J (2005) The New Zealand Marine Environment Classification. Wellington, New zealand.

Soria G, Munguía-Vega A, Marinone S, Moreno-Báez M, Martínez-Tovar I, CudneyBueno R (2012) Linking bio-oceanography and population genetics to assess larval connectivity. Marine Ecology Progress Series, 463, 159-175.

Sotka EE, Wares JP, Barth JA, Grosberg RK, Palumbi SR (2004) Strong genetic clines and geographical variation in gene flow in the rocky intertidal barnacle Balanus glandula. Molecular Ecology, 13, 2143-2156.

St-Onge P, Sévigny J-M, Strasser C, Tremblay R (2013) Strong population differentiation of softshell clams (Mya arenaria) sampled across seven biogeographic marine ecoregions: possible selection and isolation by distance. Marine Biology, 160, 10651081.

Stoms DM, Davis FW, Andelman SJ, Carr MH, Gaines SD, Halpern BS, Hoenicke R, Leibowitz SG, Leydecker A, Madin EMP, Tallis H, Warner RR (2005) Integrated coastal reserve planning: making the land-sea connection. Frontiers in Ecology and the Environment, 3, 429-436.

Strathmann RR, Hughes TP, Kuris AM, Lindeman KC, Morgan SG, Pandolfi JM, Warner RR (2002) Evolution of local recruitment and its consequences for marine populations. Bulletin of Marine Science, 70, 377-396.

Swearer SE, Shima JS, Hellberg ME, Thorrold SR, Jones GP, Robertson DR, Morgan SG, Selkoe KA, Ruiz GM, Warner RR (2002) Evidence of self-recruitment in demersal marine populations. Bulletin of Marine Science, 70, 251-271.

Sweijd NA, Bowie RCK, Evans BS, Lopata AL (2000) Molecular genetics and the management and conservation of marine organisms. Hydrobiologia, 420, 153-164.

Szostek CL, Davies AJ, Hinz H (2013) Effects of elevated levels of suspended particulate matter and burial on juvenile king scallops Pecten maximus. Marine Ecology Progress Series, 474, 155-165.

Takezaki N, Nei M, Tamura K (2014) POPTREEW: web version of POPTREE for constructing population trees from allele frequency data and computing some other quantities. Molecular Biology and Evolution, 31, 1622-4.

Talman SG, Norkko A, Thrush SF, Hewitt JE (2004) Habitat structure and the survival of juvenile scallops Pecten novaezelandiae: comparing predation in habitats with varying complexity. Marine Ecology Progress Series, 269, 197-207.

Thomas L, Bell JJ (2013) Testing the consistency of connectivity patterns for a widely dispersing marine species. Heredity, 111, 345-54. 
Thomas D, Franz B (2005) Overview of MODIS Aqua Data Processing and Distribution. NASA, National Aeronautics and Space Administration.

Thrush SF, Hewitt JE, Cummings VJ, Ellis JI, Hatton C, Lohrer A, Norkko A (2004) Muddy waters: elevating sediment input to coastal and estuarine habitats. Frontiers in Ecology and the Environment, 2, 299-306.

Toonen RJ, Andrews KR, Baums IB, Bird CE, Concepcion GT, Daly-Engel TS, Eble JA, Faucci A, Gaither MR, Iacchei M, Puritz JB, Schultz JK, Skillings DJ, Timmers MA, Bowen BW (2011) Defining Boundaries for Ecosystem-Based Management: A Multispecies Case Study of Marine Connectivity across the Hawaiian Archipelago. Journal of Marine Biology, 2011, 1-13.

Toonen RJ, Grosberg RK (2011) Causes of chaos: spatial and temporal genetic heterogeneity in the intertidal anomuran crab Petrolisthes cinctipes. In: Phylogeography and Population Genetics in Crustacea (eds Koenemann S, Held C, Schubard C), pp. 75-107. CRC Press Crustacean Issues Series.

Tuck GN, Possingham HP (2000) Marine protected areas for spatially structured exploited stocks. Marine Ecology Progress Series, 192, 89-101.

Tunbridge BR (1968) The Tasman Bay scallop fishery. N.Z. Mar. Dept. Fish. Tech. Rep. $18, \mathrm{pp} 78$.

Underwood JN, Travers MJ, Gilmour JP (2012) Subtle genetic structure reveals restricted connectivity among populations of a coral reef fish inhabiting remote atolls. Ecology and Evolution, 1-14.

Veale AJ, Lavery SD (2011) Phylogeography of the snakeskin chiton Sypharochiton pelliserpentis (Mollusca: Polyplacophora) around New Zealand: are seasonal nearshore upwelling events a dynamic barrier to gene flow? Biological Journal of the Linnean Society, 104, 552-563.

Verhoeven KJF, Simonsen KL, Mcintyre LM (2005) Implementing false discovery rate control: increasing your power. OIKOS, 3, 643-647.

Vucetich J, Waite T (2003) Spatial patterns of demography and genetic processes across the species' range: null hypotheses for landscape conservation genetics. Conservation Genetics, 4, 639-645.

Wallis GP, Trewick SA (2009) New Zealand phylogeography: evolution on a small continent. Molecular Ecology, 18, 3548-3580.

Wang IJ, Bradburd GS (2014) Isolation by Environment. Molecular Ecology, 23, 56495662 . 
Wang IJ, Summers K (2010) Genetic structure is correlated with phenotypic divergence rather than geographic isolation in the highly polymorphic strawberry poison-dart frog. Molecular Ecology, 19, 447-458.

Waples RS, Punt AE, Cope JM (2008) Integrating genetic data into management of marine resources: how can we do it better? Fish and Fisheries, 9, 423-449.

Ward RD (2000) Genetics in fisheries management. Hydrobiologia, 420, 191-201.

Watts PC, Mallanaphy PJ, Mccarthy C, Beukers-Stewart BD, Mosley MWJ, Brand AR, Saccheri IJ (2005) Polymorphic microsatellite loci isolated from the great scallop, Pecten maximus (Bivalvia: Pectinidae). Molecular Ecology Notes, 5, 902-904.

Weersing K, Toonen RJ (2009) Population genetics, larval dispersal, and connectivity in marine systems. Marine Ecology Progress Series, 393, 1-12.

Wei K, Wood AR, Gardner JPA (2013a) Population genetic variation in the New Zealand greenshell mussel: locus-dependent conflicting signals of weak structure and high gene flow balanced against pronounced structure and high self-recruitment. Marine Biology, 160, 931-949.

Wei K, Wood AR, Gardner JPA (2013b) Seascape genetics of the New Zealand greenshell mussel: sea surface temperature explains macrogeographic scale genetic variation. Marine Ecology Progress Series, 477, 107-121.

Weir BS, Cockerham CC (1984) Estimating F-Statistics for the Analysis of Population Structure. Evolution, 38, 1358-1370.

Werner FE, Cowen RK, Paris CB Coupled biological and physical models: present capabilities and necessary developments for future studies of population connectivity. Oceanography, 20, 54-69.

Westfall KM (2011) Molecular ecology and systematics of blue mussels (genus Mytilus) (Mytilidae; Bivalvia; Mollusca) in the Southern hemisphere. PhD thesis, Victoria University of Wellington.

White C, Selkoe KA, Watson J, Siegel DA, Zacherl DC, Toonen RJ (2010) Ocean currents help explain population genetic structure. Proceedings of The Royal Society Biological Sciences, 277, 1685-94.

Wilding CS, Beaumont AR, Latchford JW (1997) Mitochondrial DNA variation in the scallop Pecten maximus (L.) assessed by a PCR-RFLP method. Heredity, 79, 178-89.

Williams LR, Babcock RC (2004) Comparison of multiple techniques to evaluate reproductive variability in a marine bivalve: application to the scallop Pecten novaezelandiae. Marine and Freshwater Research, 55, 457-468. 
Williams JR, Babcock RC (2005) Assessment of size at maturity and gonad index methods for the scallop Pecten novaezelandiae. New Zealand Journal of Marine and Freshwater Research, 39, 851-864.

Williams JR, Parkinson DM (2010) Biomass survey and stock assessment for the Coromandel scallop fishery, 2010. Ministry of fisheries, Wellington.

Wood A, Gardner J (2007) Small spatial scale population genetic structure in two limpet species endemic to the Kermadec Islands, New Zealand. Marine Ecology Progress Series, 349, 159-170.

Woodburn L (1990) Genetic variation in southern Australian Pecten, in MCL Dredge, WF Zacharin \& LM Joll (eds). In: Proceedings of the Australasian Scallop Workshop, Tasmanian Government Printer, Hobart , pp. 226-240.

Worm B, Barbier EB, Beaumont N, Duffy JE, Folke C, Halpern BS, Jackson JBC, Lotze HK, Micheli F, Palumbi SR, Sala E, Selkoe KA, Stachowicz JJ, Watson R (2006) Impacts of biodiversity loss on ocean ecosystem services. Science, 314, 787-790.

Worm B, Hilborn R, Baum JK, Branch TA, Collie JS, Costello C, Fogarty MJ, Fulton EA, Hutchings JA, Jennings S, Jensen OP, Lotze HK, Mace PM, McClanahan TR, Minto C, Palumbi SR, Parma AM, Ricard D, Rosenberg AA et al. (2009) Rebuilding global fisheries. Science, 325, 578-585.

Yang Z, Nielsen R (2008) Mutation-selection models of codon substitution and their use to estimate selective strengths on codon usage. Molecular Biology and Evolution, 25, $568-579$.

Yednock BK, Neigel JE (2014) An investigation of genetic population structure in blue crabs, Callinectes sapidus, using nuclear gene sequences. Marine Biology, 161, 871886.

Zeldis JR (2004) New and remineralised nutrient supply and ecosystem metabolism on the northeastern New Zealand continental shelf. Continental Shelf Research, 24, 563-581.

Zeldis JR, Walters RA, Greig MJN, Image K (2004) Circulation over the northeastern New Zealand continental slope, shelf and adjacent Hauraki Gulf, during spring and summer. Continental Shelf Research, 24, 543-561.

Zhan A, Hu J, Hu X, Zhou Z, Hui M, Wang S, Peng W, Wang M, Bao Z (2009) Fine-scale population genetic structure of Zhikong scallop (Chlamys farreri): do local marine currents drive geographical differentiation? Marine Biotechnology, 11, 223-35. 


\section{Appendices}

Supplementary Table 1 PCR conditions for 12 microsatellite loci of Pecten novaezelandiae (Chapter 2).

\begin{tabular}{|c|c|c|c|c|c|}
\hline $\begin{array}{l}\text { Multiplex } \\
\text { group }\end{array}$ & Locus & Repeat & Forward primer sequence $\left(5^{\prime}-3^{\prime}\right)$ & Dye & Cycling conditions \\
\hline \multicolumn{6}{|l|}{1 (Pool 1) } \\
\hline & Pnova_09 & $(\mathrm{AGTT})_{5}$ & GGCGTCCACTGACAGATAAG & VIC & \\
\hline & Pnova_24 & $(\mathrm{AGAC})_{5}$ & CACTGACGAAACGTTGGTGT & PET & \\
\hline \multirow[t]{3}{*}{2 (Pool 1) } & Pnova_02 & $(\mathrm{TA})_{11}$ & CAGTAGCTCCTGCCCCATTA & 6-FAM & $94^{\circ} \mathrm{C}(5 \mathrm{~min})$ \\
\hline & Pnova_10 & $(\mathrm{TA})_{11}$ & GGAAGGAAGCACAGCAGGTA & 6-FAM & 36 cycles at \\
\hline & Pnova_27 & $(\text { TATC })_{33}$ & CAGATACGTCAGAGTGCTGATTC & NED & $94^{\circ} \mathrm{C}(30 \mathrm{~s}) / 61^{\circ} \mathrm{C}(45 \mathrm{~s}) / 74^{\circ} \mathrm{C}(60 \mathrm{~s})$ \\
\hline \multirow[t]{3}{*}{3 (Pool 1) } & Pnova_01 & $(\mathrm{TA})_{8}$ & GTAGCACACGCAAATGCCTA & 6-FAM & $74^{\circ} \mathrm{C}(10 \mathrm{~min})$ \\
\hline & Pnova_04 & $(\mathrm{TTGT})_{7}$ & AATGCAGGAAGGTTTTGGTTT & PET & \\
\hline & Pnova_28 & $(\mathrm{CAA})_{19}$ & AAGGCATATAACTCAGCTGGAA & VIC & \\
\hline \multirow[t]{4}{*}{$4($ Pool 2) } & Pnova_05 & $(\mathrm{ACAG})_{7}$ & AGCGACATACAATGGGGAAG & VIC & $94^{\circ} \mathrm{C}(5 \mathrm{~min})$ \\
\hline & Pnova_31 & $(\mathrm{TA})_{11}$ & TGCGCTACAGTGTCGTTCTT & 6-FAM & 36 cycles at \\
\hline & Pnova_32 & $(\mathrm{AT})_{11}$ & GTGTCGTCTACAGCCGGAAT & NED & $94^{\circ} \mathrm{C}(30 \mathrm{~s}) / 62^{\circ} \mathrm{C}(45 \mathrm{~s}) / 74^{\circ} \mathrm{C}(60 \mathrm{~s})$ \\
\hline & Pnova_33 & $\begin{array}{c}(\mathrm{AATAT})_{1} \\
0\end{array}$ & CTCCAGGAGTAGAGCCGATG & PET & $74^{\circ} \mathrm{C}(10 \mathrm{~min})$ \\
\hline
\end{tabular}



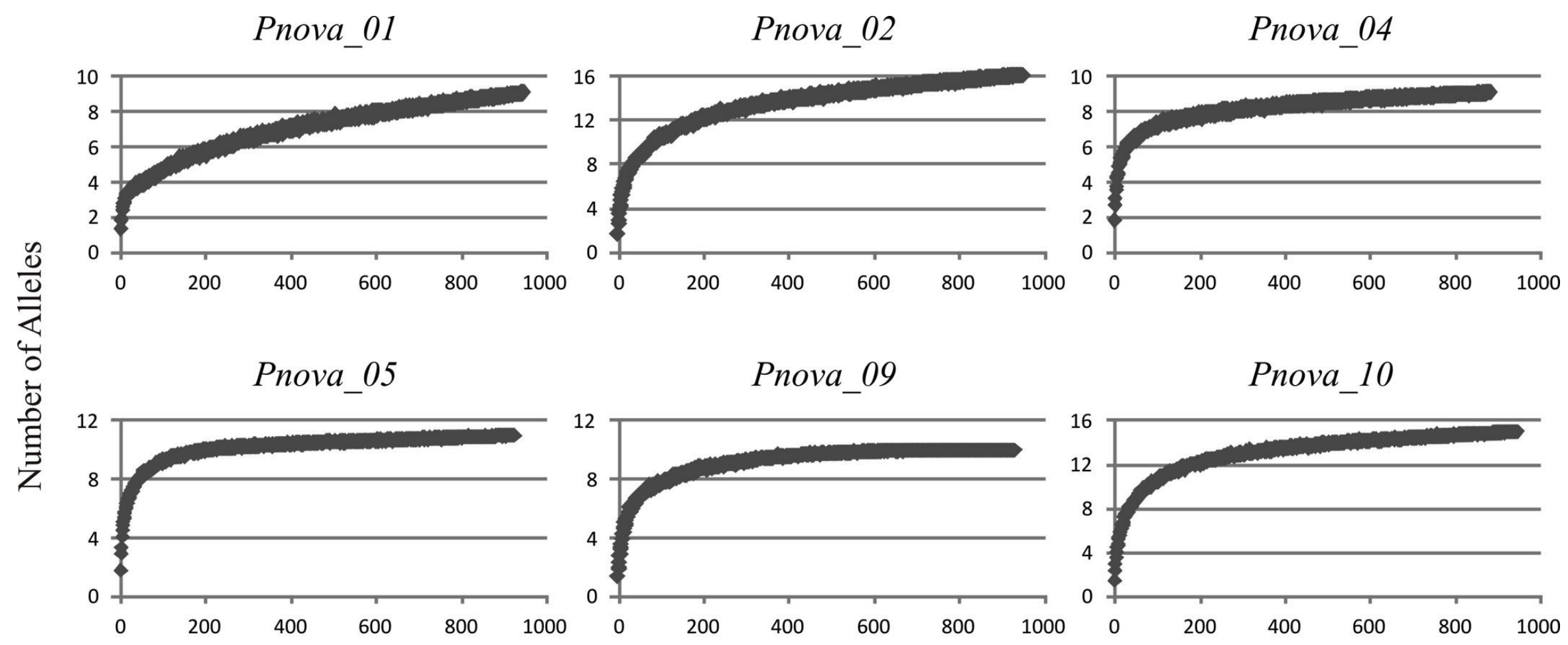

Number of individuals

Supplementary Figure 1 Allele discovery curves for Pecten novaezelandiae (Chapter 3). 

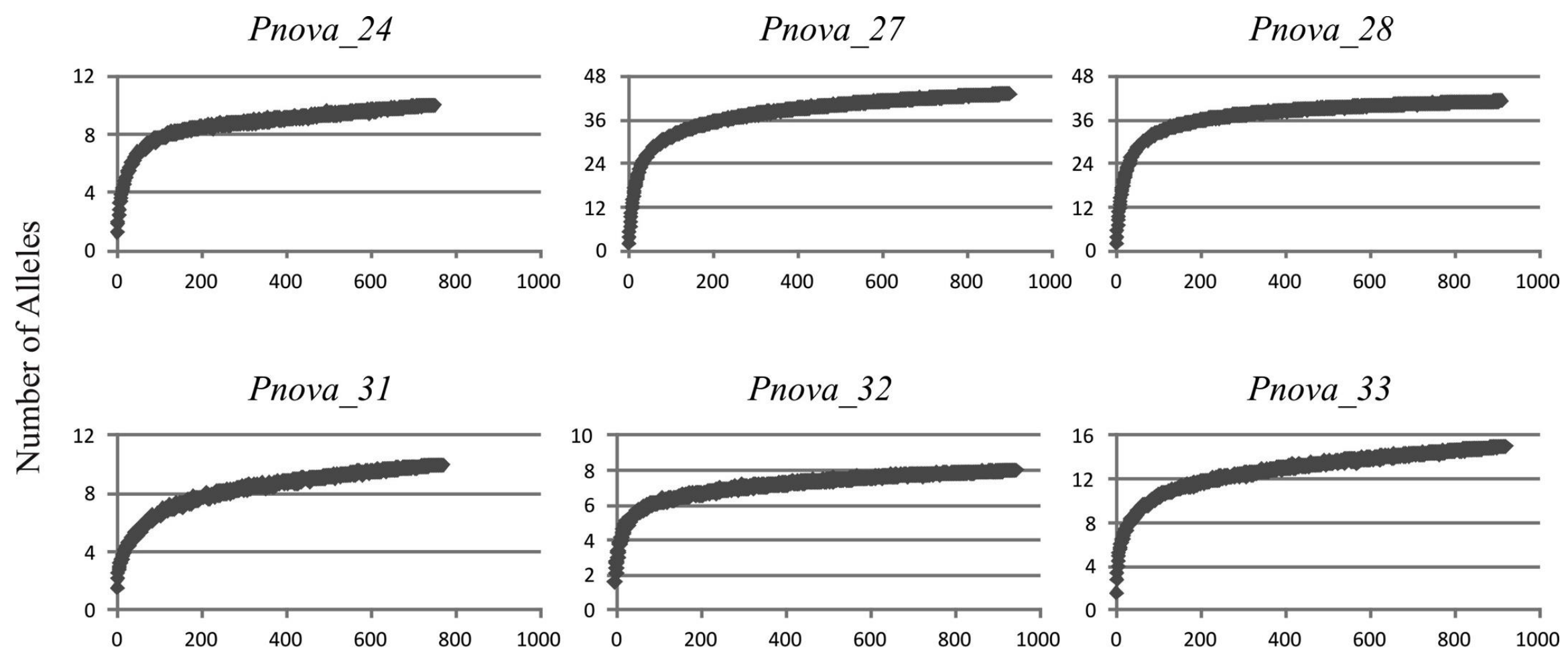

Number of individuals

Supplementary Figure 1 (Cont.) 
Supplementary Table 2 Allele frequencies for 10 microsatellite loci of Pecten novaezelandiae for 15 locations (Chapter 3 ).

\begin{tabular}{|c|c|c|c|c|c|c|c|c|c|c|c|c|c|c|c|c|}
\hline Locus & Allele & RAN & LBI & JON & HAU & MER & TAU & WEL12 & WEL13 & MAR & TAS & GOL & FIO & STE12 & STE14 & СНА \\
\hline \multirow[t]{9}{*}{ Pnova_01 } & 207 & 0.000 & 0.011 & 0.000 & 0.000 & 0.000 & 0.000 & 0.007 & 0.021 & 0.000 & 0.000 & 0.000 & 0.000 & 0.000 & 0.000 & 0.000 \\
\hline & 217 & 0.000 & 0.000 & 0.013 & 0.000 & 0.000 & 0.000 & 0.000 & 0.000 & 0.000 & 0.000 & 0.000 & 0.000 & 0.000 & 0.000 & 0.000 \\
\hline & 221 & 0.143 & 0.101 & 0.050 & 0.121 & 0.123 & 0.051 & 0.075 & 0.135 & 0.198 & 0.115 & 0.094 & 0.206 & 0.200 & 0.281 & 0.087 \\
\hline & 223 & 0.832 & 0.878 & 0.800 & 0.869 & 0.848 & 0.918 & 0.896 & 0.802 & 0.771 & 0.854 & 0.885 & 0.794 & 0.800 & 0.719 & 0.903 \\
\hline & 225 & 0.026 & 0.005 & 0.113 & 0.005 & 0.029 & 0.031 & 0.015 & 0.021 & 0.010 & 0.031 & 0.021 & 0.000 & 0.000 & 0.000 & 0.005 \\
\hline & 227 & 0.000 & 0.000 & 0.025 & 0.000 & 0.000 & 0.000 & 0.007 & 0.010 & 0.010 & 0.000 & 0.000 & 0.000 & 0.000 & 0.000 & 0.000 \\
\hline & 229 & 0.000 & 0.005 & 0.000 & 0.000 & 0.000 & 0.000 & 0.000 & 0.000 & 0.000 & 0.000 & 0.000 & 0.000 & 0.000 & 0.000 & 0.000 \\
\hline & 231 & 0.000 & 0.000 & 0.000 & 0.005 & 0.000 & 0.000 & 0.000 & 0.000 & 0.000 & 0.000 & 0.000 & 0.000 & 0.000 & 0.000 & 0.005 \\
\hline & 233 & 0.000 & 0.000 & 0.000 & 0.000 & 0.000 & 0.000 & 0.000 & 0.010 & 0.010 & 0.000 & 0.000 & 0.000 & 0.000 & 0.000 & 0.000 \\
\hline \multirow[t]{16}{*}{ Pnova_02 } & 153 & 0.005 & 0.000 & 0.000 & 0.000 & 0.000 & 0.000 & 0.000 & 0.000 & 0.000 & 0.000 & 0.000 & 0.000 & 0.000 & 0.000 & 0.000 \\
\hline & 157 & 0.000 & 0.000 & 0.000 & 0.000 & 0.000 & 0.000 & 0.000 & 0.000 & 0.000 & 0.000 & 0.000 & 0.000 & 0.000 & 0.000 & 0.005 \\
\hline & 159 & 0.000 & 0.000 & 0.000 & 0.000 & 0.000 & 0.000 & 0.000 & 0.000 & 0.010 & 0.000 & 0.000 & 0.014 & 0.000 & 0.000 & 0.000 \\
\hline & 161 & 0.005 & 0.000 & 0.000 & 0.010 & 0.007 & 0.000 & 0.000 & 0.032 & 0.000 & 0.010 & 0.021 & 0.000 & 0.000 & 0.052 & 0.010 \\
\hline & 163 & 0.005 & 0.000 & 0.013 & 0.000 & 0.000 & 0.010 & 0.007 & 0.000 & 0.000 & 0.000 & 0.010 & 0.000 & 0.000 & 0.000 & 0.000 \\
\hline & 165 & 0.015 & 0.021 & 0.013 & 0.025 & 0.036 & 0.035 & 0.045 & 0.021 & 0.040 & 0.042 & 0.031 & 0.214 & 0.050 & 0.094 & 0.015 \\
\hline & 167 & 0.250 & 0.266 & 0.295 & 0.197 & 0.239 & 0.288 & 0.239 & 0.191 & 0.250 & 0.250 & 0.188 & 0.257 & 0.150 & 0.208 & 0.245 \\
\hline & 169 & 0.505 & 0.479 & 0.500 & 0.556 & 0.522 & 0.510 & 0.470 & 0.564 & 0.540 & 0.490 & 0.458 & 0.414 & 0.650 & 0.469 & 0.587 \\
\hline & 171 & 0.061 & 0.069 & 0.090 & 0.045 & 0.094 & 0.045 & 0.090 & 0.106 & 0.060 & 0.073 & 0.104 & 0.043 & 0.100 & 0.125 & 0.102 \\
\hline & 173 & 0.097 & 0.128 & 0.000 & 0.101 & 0.065 & 0.051 & 0.067 & 0.032 & 0.050 & 0.083 & 0.094 & 0.000 & 0.000 & 0.000 & 0.000 \\
\hline & 175 & 0.031 & 0.016 & 0.064 & 0.035 & 0.014 & 0.030 & 0.060 & 0.043 & 0.050 & 0.042 & 0.073 & 0.043 & 0.000 & 0.052 & 0.031 \\
\hline & 177 & 0.015 & 0.011 & 0.026 & 0.005 & 0.014 & 0.010 & 0.000 & 0.011 & 0.000 & 0.000 & 0.010 & 0.000 & 0.000 & 0.000 & 0.000 \\
\hline & 179 & 0.000 & 0.011 & 0.000 & 0.010 & 0.007 & 0.010 & 0.000 & 0.000 & 0.000 & 0.010 & 0.010 & 0.000 & 0.000 & 0.000 & 0.000 \\
\hline & 181 & 0.005 & 0.000 & 0.000 & 0.010 & 0.000 & 0.005 & 0.015 & 0.000 & 0.000 & 0.000 & 0.000 & 0.014 & 0.050 & 0.000 & 0.000 \\
\hline & 183 & 0.000 & 0.000 & 0.000 & 0.005 & 0.000 & 0.000 & 0.000 & 0.000 & 0.000 & 0.000 & 0.000 & 0.000 & 0.000 & 0.000 & 0.000 \\
\hline & 185 & 0.005 & 0.000 & 0.000 & 0.000 & 0.000 & 0.005 & 0.007 & 0.000 & 0.000 & 0.000 & 0.000 & 0.000 & 0.000 & 0.000 & 0.005 \\
\hline
\end{tabular}


Supplementary Table 2 (cont.)

\begin{tabular}{|c|c|c|c|c|c|c|c|c|c|c|c|c|c|c|c|c|}
\hline Locus & Allele & RAN & LBI & JON & HAU & MER & TAU & WEL12 & WEL13 & MAR & TAS & GOL & FIO & STE12 & STE14 & CHA \\
\hline \multirow[t]{9}{*}{ Pnova_04 } & 272 & 0.000 & 0.000 & 0.000 & 0.000 & 0.007 & 0.000 & 0.000 & 0.000 & 0.000 & 0.000 & 0.000 & 0.000 & 0.000 & 0.000 & 0.000 \\
\hline & 276 & 0.137 & 0.131 & 0.163 & 0.148 & 0.104 & 0.117 & 0.185 & 0.159 & 0.288 & 0.192 & 0.211 & 0.157 & 0.000 & 0.125 & 0.087 \\
\hline & 280 & 0.005 & 0.023 & 0.013 & 0.022 & 0.007 & 0.010 & 0.000 & 0.000 & 0.013 & 0.000 & 0.000 & 0.043 & 0.000 & 0.021 & 0.031 \\
\hline & 284 & 0.089 & 0.097 & 0.063 & 0.099 & 0.030 & 0.071 & 0.083 & 0.037 & 0.050 & 0.051 & 0.053 & 0.043 & 0.300 & 0.167 & 0.138 \\
\hline & 288 & 0.500 & 0.511 & 0.525 & 0.495 & 0.634 & 0.561 & 0.444 & 0.573 & 0.450 & 0.577 & 0.513 & 0.471 & 0.450 & 0.354 & 0.367 \\
\hline & 292 & 0.211 & 0.193 & 0.200 & 0.192 & 0.179 & 0.199 & 0.269 & 0.220 & 0.125 & 0.154 & 0.171 & 0.214 & 0.200 & 0.323 & 0.316 \\
\hline & 296 & 0.053 & 0.034 & 0.025 & 0.044 & 0.022 & 0.031 & 0.019 & 0.012 & 0.038 & 0.013 & 0.039 & 0.071 & 0.050 & 0.010 & 0.041 \\
\hline & 300 & 0.005 & 0.011 & 0.013 & 0.000 & 0.007 & 0.005 & 0.000 & 0.000 & 0.025 & 0.013 & 0.013 & 0.000 & 0.000 & 0.000 & 0.020 \\
\hline & 304 & 0.000 & 0.000 & 0.000 & 0.000 & 0.007 & 0.005 & 0.000 & 0.000 & 0.013 & 0.000 & 0.000 & 0.000 & 0.000 & 0.000 & 0.000 \\
\hline \multirow[t]{11}{*}{ Pnova_05 } & 131 & 0.036 & 0.043 & 0.033 & 0.052 & 0.059 & 0.041 & 0.022 & 0.031 & 0.020 & 0.010 & 0.021 & 0.000 & 0.000 & 0.010 & 0.026 \\
\hline & 135 & 0.117 & 0.161 & 0.117 & 0.063 & 0.096 & 0.112 & 0.164 & 0.094 & 0.130 & 0.177 & 0.115 & 0.129 & 0.000 & 0.198 & 0.149 \\
\hline & 139 & 0.168 & 0.140 & 0.167 & 0.092 & 0.154 & 0.168 & 0.104 & 0.198 & 0.080 & 0.167 & 0.125 & 0.100 & 0.200 & 0.156 & 0.180 \\
\hline & 143 & 0.357 & 0.403 & 0.233 & 0.385 & 0.397 & 0.434 & 0.358 & 0.271 & 0.360 & 0.375 & 0.469 & 0.486 & 0.300 & 0.344 & 0.371 \\
\hline & 147 & 0.240 & 0.167 & 0.300 & 0.293 & 0.213 & 0.163 & 0.246 & 0.302 & 0.290 & 0.188 & 0.229 & 0.243 & 0.400 & 0.198 & 0.216 \\
\hline & 151 & 0.046 & 0.054 & 0.067 & 0.075 & 0.051 & 0.046 & 0.067 & 0.063 & 0.060 & 0.042 & 0.021 & 0.000 & 0.100 & 0.042 & 0.031 \\
\hline & 155 & 0.020 & 0.016 & 0.033 & 0.011 & 0.029 & 0.015 & 0.022 & 0.010 & 0.010 & 0.031 & 0.010 & 0.014 & 0.000 & 0.031 & 0.010 \\
\hline & 159 & 0.005 & 0.005 & 0.000 & 0.011 & 0.000 & 0.000 & 0.015 & 0.010 & 0.020 & 0.000 & 0.010 & 0.029 & 0.000 & 0.010 & 0.005 \\
\hline & 163 & 0.010 & 0.011 & 0.050 & 0.000 & 0.000 & 0.010 & 0.000 & 0.010 & 0.010 & 0.000 & 0.000 & 0.000 & 0.000 & 0.010 & 0.005 \\
\hline & 167 & 0.000 & 0.000 & 0.000 & 0.017 & 0.000 & 0.005 & 0.000 & 0.010 & 0.020 & 0.010 & 0.000 & 0.000 & 0.000 & 0.000 & 0.005 \\
\hline & 171 & 0.000 & 0.000 & 0.000 & 0.000 & 0.000 & 0.005 & 0.000 & 0.000 & 0.000 & 0.000 & 0.000 & 0.000 & 0.000 & 0.000 & 0.000 \\
\hline \multirow[t]{4}{*}{ Pnova_09 } & 104 & 0.020 & 0.000 & 0.000 & 0.000 & 0.000 & 0.000 & 0.000 & 0.000 & 0.000 & 0.000 & 0.000 & 0.000 & 0.000 & 0.000 & 0.000 \\
\hline & 108 & 0.000 & 0.000 & 0.000 & 0.000 & 0.000 & 0.000 & 0.000 & 0.000 & 0.000 & 0.000 & 0.000 & 0.000 & 0.000 & 0.034 & 0.000 \\
\hline & 112 & 0.015 & 0.043 & 0.013 & 0.046 & 0.030 & 0.026 & 0.007 & 0.011 & 0.000 & 0.000 & 0.021 & 0.015 & 0.000 & 0.000 & 0.016 \\
\hline & 116 & 0.051 & 0.033 & 0.038 & 0.056 & 0.075 & 0.052 & 0.030 & 0.022 & 0.043 & 0.000 & 0.031 & 0.000 & 0.150 & 0.011 & 0.053 \\
\hline
\end{tabular}


Supplementary Table 2 (cont.)

\begin{tabular}{|c|c|c|c|c|c|c|c|c|c|c|c|c|c|c|c|c|}
\hline Locus & Allele & RAN & LBI & JON & HAU & MER & TAU & WEL12 & WEL13 & MAR & TAS & GOL & FIO & STE12 & STE14 & CHA \\
\hline \multirow[t]{6}{*}{ Pnova_09 } & 120 & 0.740 & 0.766 & 0.800 & 0.724 & 0.679 & 0.742 & 0.888 & 0.880 & 0.840 & 0.885 & 0.885 & 0.882 & 0.800 & 0.898 & 0.700 \\
\hline & 124 & 0.051 & 0.054 & 0.063 & 0.066 & 0.090 & 0.052 & 0.052 & 0.054 & 0.053 & 0.083 & 0.042 & 0.000 & 0.050 & 0.023 & 0.147 \\
\hline & 128 & 0.092 & 0.087 & 0.063 & 0.082 & 0.082 & 0.098 & 0.015 & 0.011 & 0.000 & 0.021 & 0.010 & 0.015 & 0.000 & 0.000 & 0.063 \\
\hline & 132 & 0.020 & 0.011 & 0.025 & 0.026 & 0.030 & 0.015 & 0.000 & 0.011 & 0.043 & 0.000 & 0.010 & 0.088 & 0.000 & 0.011 & 0.011 \\
\hline & 136 & 0.005 & 0.000 & 0.000 & 0.000 & 0.007 & 0.010 & 0.000 & 0.011 & 0.011 & 0.010 & 0.000 & 0.000 & 0.000 & 0.023 & 0.011 \\
\hline & 144 & 0.005 & 0.005 & 0.000 & 0.000 & 0.007 & 0.005 & 0.007 & 0.000 & 0.011 & 0.000 & 0.000 & 0.000 & 0.000 & 0.000 & 0.000 \\
\hline \multirow[t]{13}{*}{ Pnova_10 } & 254 & 0.010 & 0.000 & 0.000 & 0.000 & 0.000 & 0.000 & 0.000 & 0.000 & 0.000 & 0.000 & 0.000 & 0.000 & 0.000 & 0.000 & 0.000 \\
\hline & 256 & 0.021 & 0.000 & 0.026 & 0.000 & 0.000 & 0.000 & 0.000 & 0.010 & 0.000 & 0.000 & 0.000 & 0.000 & 0.000 & 0.000 & 0.005 \\
\hline & 258 & 0.026 & 0.043 & 0.051 & 0.041 & 0.058 & 0.027 & 0.015 & 0.052 & 0.020 & 0.010 & 0.042 & 0.000 & 0.000 & 0.010 & 0.010 \\
\hline & 260 & 0.067 & 0.092 & 0.090 & 0.107 & 0.101 & 0.048 & 0.112 & 0.094 & 0.120 & 0.156 & 0.177 & 0.338 & 0.188 & 0.188 & 0.179 \\
\hline & 262 & 0.010 & 0.033 & 0.000 & 0.046 & 0.007 & 0.011 & 0.067 & 0.052 & 0.040 & 0.021 & 0.063 & 0.000 & 0.000 & 0.000 & 0.015 \\
\hline & 264 & 0.273 & 0.293 & 0.372 & 0.245 & 0.254 & 0.293 & 0.194 & 0.219 & 0.340 & 0.344 & 0.208 & 0.147 & 0.375 & 0.240 & 0.245 \\
\hline & 266 & 0.438 & 0.397 & 0.372 & 0.434 & 0.420 & 0.426 & 0.463 & 0.448 & 0.430 & 0.385 & 0.406 & 0.500 & 0.438 & 0.500 & 0.444 \\
\hline & 268 & 0.108 & 0.109 & 0.064 & 0.097 & 0.138 & 0.138 & 0.112 & 0.115 & 0.050 & 0.052 & 0.063 & 0.015 & 0.000 & 0.042 & 0.061 \\
\hline & 270 & 0.026 & 0.016 & 0.013 & 0.005 & 0.007 & 0.016 & 0.037 & 0.010 & 0.000 & 0.010 & 0.031 & 0.000 & 0.000 & 0.010 & 0.036 \\
\hline & 272 & 0.005 & 0.005 & 0.000 & 0.020 & 0.000 & 0.005 & 0.000 & 0.000 & 0.000 & 0.021 & 0.000 & 0.000 & 0.000 & 0.000 & 0.000 \\
\hline & 274 & 0.015 & 0.005 & 0.013 & 0.005 & 0.000 & 0.027 & 0.000 & 0.000 & 0.000 & 0.000 & 0.000 & 0.000 & 0.000 & 0.000 & 0.005 \\
\hline & 278 & 0.000 & 0.005 & 0.000 & 0.000 & 0.007 & 0.011 & 0.000 & 0.000 & 0.000 & 0.000 & 0.010 & 0.000 & 0.000 & 0.010 & 0.000 \\
\hline & 280 & 0.000 & 0.000 & 0.000 & 0.000 & 0.007 & 0.000 & 0.000 & 0.000 & 0.000 & 0.000 & 0.000 & 0.000 & 0.000 & 0.000 & 0.000 \\
\hline \multirow[t]{8}{*}{ Pnova_27 } & 151 & 0.000 & 0.011 & 0.000 & 0.005 & 0.000 & 0.000 & 0.000 & 0.000 & 0.000 & 0.000 & 0.000 & 0.000 & 0.000 & 0.000 & 0.000 \\
\hline & 167 & 0.000 & 0.000 & 0.000 & 0.000 & 0.008 & 0.000 & 0.000 & 0.000 & 0.000 & 0.000 & 0.000 & 0.000 & 0.000 & 0.000 & 0.000 \\
\hline & 171 & 0.000 & 0.000 & 0.000 & 0.011 & 0.000 & 0.000 & 0.000 & 0.000 & 0.000 & 0.000 & 0.000 & 0.000 & 0.000 & 0.000 & 0.000 \\
\hline & 183 & 0.018 & 0.000 & 0.000 & 0.005 & 0.000 & 0.000 & 0.000 & 0.000 & 0.000 & 0.000 & 0.000 & 0.000 & 0.000 & 0.000 & 0.000 \\
\hline & 187 & 0.006 & 0.005 & 0.000 & 0.005 & 0.000 & 0.000 & 0.000 & 0.022 & 0.000 & 0.011 & 0.011 & 0.000 & 0.000 & 0.000 & 0.000 \\
\hline & 191 & 0.006 & 0.011 & 0.028 & 0.016 & 0.000 & 0.011 & 0.008 & 0.011 & 0.010 & 0.000 & 0.000 & 0.000 & 0.000 & 0.011 & 0.000 \\
\hline & 195 & 0.006 & 0.005 & 0.028 & 0.005 & 0.008 & 0.005 & 0.031 & 0.000 & 0.010 & 0.021 & 0.011 & 0.000 & 0.000 & 0.021 & 0.028 \\
\hline & 199 & 0.006 & 0.021 & 0.014 & 0.026 & 0.030 & 0.027 & 0.008 & 0.000 & 0.000 & 0.021 & 0.043 & 0.000 & 0.000 & 0.011 & 0.006 \\
\hline
\end{tabular}


Supplementary Table 2 (cont.)

\begin{tabular}{|c|c|c|c|c|c|c|c|c|c|c|c|c|c|c|c|c|}
\hline Locus & Allele & RAN & LBI & JON & HAU & MER & TAU & WEL12 & WEL13 & MAR & TAS & GOL & FIO & STE12 & STE14 & CHA \\
\hline \multirow[t]{27}{*}{ Pnova_27 } & 203 & 0.018 & 0.005 & 0.014 & 0.021 & 0.061 & 0.011 & 0.015 & 0.011 & 0.010 & 0.011 & 0.000 & 0.000 & 0.000 & 0.011 & 0.044 \\
\hline & 207 & 0.029 & 0.032 & 0.014 & 0.032 & 0.023 & 0.049 & 0.069 & 0.022 & 0.063 & 0.011 & 0.043 & 0.086 & 0.050 & 0.021 & 0.050 \\
\hline & 211 & 0.029 & 0.016 & 0.014 & 0.047 & 0.015 & 0.038 & 0.038 & 0.011 & 0.052 & 0.032 & 0.033 & 0.129 & 0.100 & 0.021 & 0.083 \\
\hline & 215 & 0.035 & 0.032 & 0.042 & 0.021 & 0.015 & 0.049 & 0.031 & 0.033 & 0.063 & 0.011 & 0.033 & 0.057 & 0.100 & 0.074 & 0.078 \\
\hline & 219 & 0.024 & 0.069 & 0.042 & 0.026 & 0.030 & 0.055 & 0.031 & 0.065 & 0.052 & 0.032 & 0.054 & 0.057 & 0.000 & 0.085 & 0.044 \\
\hline & 223 & 0.012 & 0.053 & 0.014 & 0.032 & 0.061 & 0.066 & 0.054 & 0.054 & 0.052 & 0.064 & 0.011 & 0.057 & 0.150 & 0.128 & 0.050 \\
\hline & 227 & 0.065 & 0.048 & 0.111 & 0.053 & 0.030 & 0.060 & 0.023 & 0.043 & 0.042 & 0.085 & 0.043 & 0.100 & 0.050 & 0.043 & 0.056 \\
\hline & 231 & 0.053 & 0.064 & 0.042 & 0.053 & 0.061 & 0.049 & 0.038 & 0.022 & 0.094 & 0.032 & 0.011 & 0.086 & 0.100 & 0.096 & 0.089 \\
\hline & 235 & 0.053 & 0.064 & 0.042 & 0.047 & 0.053 & 0.044 & 0.062 & 0.076 & 0.083 & 0.064 & 0.054 & 0.086 & 0.000 & 0.053 & 0.100 \\
\hline & 239 & 0.059 & 0.059 & 0.042 & 0.037 & 0.053 & 0.071 & 0.054 & 0.022 & 0.021 & 0.074 & 0.054 & 0.057 & 0.050 & 0.064 & 0.061 \\
\hline & 243 & 0.106 & 0.059 & 0.097 & 0.042 & 0.053 & 0.077 & 0.031 & 0.033 & 0.073 & 0.043 & 0.022 & 0.086 & 0.050 & 0.053 & 0.061 \\
\hline & 247 & 0.076 & 0.064 & 0.069 & 0.079 & 0.023 & 0.071 & 0.031 & 0.065 & 0.031 & 0.096 & 0.130 & 0.057 & 0.000 & 0.053 & 0.033 \\
\hline & 251 & 0.041 & 0.032 & 0.069 & 0.063 & 0.053 & 0.077 & 0.077 & 0.011 & 0.031 & 0.053 & 0.043 & 0.014 & 0.100 & 0.074 & 0.056 \\
\hline & 255 & 0.041 & 0.016 & 0.014 & 0.074 & 0.076 & 0.049 & 0.023 & 0.043 & 0.073 & 0.043 & 0.065 & 0.071 & 0.000 & 0.074 & 0.056 \\
\hline & 259 & 0.065 & 0.059 & 0.083 & 0.053 & 0.061 & 0.027 & 0.038 & 0.033 & 0.042 & 0.074 & 0.033 & 0.014 & 0.000 & 0.043 & 0.028 \\
\hline & 263 & 0.029 & 0.048 & 0.111 & 0.063 & 0.083 & 0.044 & 0.054 & 0.033 & 0.031 & 0.032 & 0.065 & 0.029 & 0.100 & 0.011 & 0.022 \\
\hline & 267 & 0.035 & 0.037 & 0.028 & 0.032 & 0.030 & 0.016 & 0.038 & 0.065 & 0.000 & 0.043 & 0.033 & 0.000 & 0.100 & 0.021 & 0.022 \\
\hline & 271 & 0.035 & 0.043 & 0.014 & 0.026 & 0.023 & 0.033 & 0.038 & 0.043 & 0.021 & 0.053 & 0.000 & 0.000 & 0.000 & 0.011 & 0.006 \\
\hline & 275 & 0.035 & 0.027 & 0.028 & 0.026 & 0.030 & 0.016 & 0.046 & 0.043 & 0.031 & 0.011 & 0.022 & 0.000 & 0.000 & 0.000 & 0.011 \\
\hline & 279 & 0.035 & 0.043 & 0.014 & 0.005 & 0.023 & 0.005 & 0.023 & 0.000 & 0.021 & 0.032 & 0.033 & 0.014 & 0.000 & 0.000 & 0.017 \\
\hline & 283 & 0.029 & 0.016 & 0.028 & 0.042 & 0.053 & 0.005 & 0.031 & 0.011 & 0.031 & 0.011 & 0.022 & 0.000 & 0.000 & 0.000 & 0.000 \\
\hline & 287 & 0.024 & 0.005 & 0.000 & 0.011 & 0.000 & 0.005 & 0.015 & 0.076 & 0.021 & 0.032 & 0.022 & 0.000 & 0.000 & 0.011 & 0.000 \\
\hline & 291 & 0.006 & 0.016 & 0.000 & 0.011 & 0.015 & 0.005 & 0.023 & 0.011 & 0.000 & 0.000 & 0.033 & 0.000 & 0.050 & 0.000 & 0.000 \\
\hline & 295 & 0.000 & 0.005 & 0.000 & 0.005 & 0.008 & 0.000 & 0.008 & 0.033 & 0.031 & 0.000 & 0.043 & 0.000 & 0.000 & 0.011 & 0.000 \\
\hline & 299 & 0.000 & 0.011 & 0.000 & 0.005 & 0.015 & 0.005 & 0.000 & 0.033 & 0.010 & 0.000 & 0.022 & 0.000 & 0.000 & 0.000 & 0.000 \\
\hline & 303 & 0.006 & 0.005 & 0.000 & 0.000 & 0.000 & 0.000 & 0.008 & 0.022 & 0.000 & 0.000 & 0.000 & 0.000 & 0.000 & 0.000 & 0.000 \\
\hline & 307 & 0.012 & 0.000 & 0.000 & 0.011 & 0.000 & 0.000 & 0.008 & 0.033 & 0.000 & 0.000 & 0.000 & 0.000 & 0.000 & 0.000 & 0.000 \\
\hline
\end{tabular}


Supplementary Table 2 (cont.)

\begin{tabular}{|c|c|c|c|c|c|c|c|c|c|c|c|c|c|c|c|c|}
\hline Locus & Allele & RAN & LBI & JON & HAU & MER & TAU & WEL12 & WEL13 & MAR & TAS & GOL & FIO & STE12 & STE14 & CHA \\
\hline \multirow[t]{8}{*}{ Pnova_27 } & 311 & 0.000 & 0.011 & 0.000 & 0.011 & 0.000 & 0.005 & 0.000 & 0.000 & 0.000 & 0.000 & 0.011 & 0.000 & 0.000 & 0.000 & 0.000 \\
\hline & 315 & 0.000 & 0.000 & 0.000 & 0.000 & 0.000 & 0.011 & 0.015 & 0.011 & 0.000 & 0.000 & 0.000 & 0.000 & 0.000 & 0.000 & 0.000 \\
\hline & 319 & 0.000 & 0.000 & 0.000 & 0.000 & 0.000 & 0.000 & 0.015 & 0.000 & 0.000 & 0.011 & 0.000 & 0.000 & 0.000 & 0.000 & 0.000 \\
\hline & 323 & 0.000 & 0.011 & 0.000 & 0.000 & 0.000 & 0.000 & 0.000 & 0.011 & 0.000 & 0.000 & 0.000 & 0.000 & 0.000 & 0.000 & 0.000 \\
\hline & 327 & 0.000 & 0.000 & 0.000 & 0.000 & 0.000 & 0.005 & 0.008 & 0.000 & 0.000 & 0.000 & 0.000 & 0.000 & 0.000 & 0.000 & 0.000 \\
\hline & 339 & 0.006 & 0.000 & 0.000 & 0.000 & 0.000 & 0.000 & 0.000 & 0.000 & 0.000 & 0.000 & 0.000 & 0.000 & 0.000 & 0.000 & 0.000 \\
\hline & 343 & 0.000 & 0.000 & 0.000 & 0.000 & 0.000 & 0.000 & 0.008 & 0.000 & 0.000 & 0.000 & 0.000 & 0.000 & 0.000 & 0.000 & 0.000 \\
\hline & 379 & 0.000 & 0.000 & 0.000 & 0.000 & 0.008 & 0.000 & 0.000 & 0.000 & 0.000 & 0.000 & 0.000 & 0.000 & 0.000 & 0.000 & 0.000 \\
\hline \multirow[t]{19}{*}{ Pnova_28 } & 248 & 0.006 & 0.000 & 0.000 & 0.000 & 0.000 & 0.005 & 0.000 & 0.000 & 0.000 & 0.000 & 0.000 & 0.000 & 0.000 & 0.000 & 0.000 \\
\hline & 251 & 0.000 & 0.000 & 0.000 & 0.000 & 0.000 & 0.000 & 0.000 & 0.000 & 0.000 & 0.010 & 0.000 & 0.000 & 0.000 & 0.000 & 0.000 \\
\hline & 254 & 0.022 & 0.022 & 0.025 & 0.011 & 0.031 & 0.022 & 0.008 & 0.021 & 0.021 & 0.042 & 0.021 & 0.074 & 0.167 & 0.083 & 0.047 \\
\hline & 257 & 0.011 & 0.000 & 0.013 & 0.000 & 0.000 & 0.000 & 0.000 & 0.000 & 0.000 & 0.000 & 0.000 & 0.000 & 0.000 & 0.000 & 0.026 \\
\hline & 263 & 0.000 & 0.000 & 0.000 & 0.000 & 0.000 & 0.000 & 0.000 & 0.011 & 0.000 & 0.000 & 0.000 & 0.000 & 0.000 & 0.000 & 0.000 \\
\hline & 266 & 0.000 & 0.005 & 0.013 & 0.000 & 0.008 & 0.000 & 0.008 & 0.032 & 0.010 & 0.021 & 0.000 & 0.000 & 0.000 & 0.010 & 0.000 \\
\hline & 269 & 0.000 & 0.022 & 0.000 & 0.000 & 0.000 & 0.000 & 0.000 & 0.000 & 0.000 & 0.000 & 0.000 & 0.000 & 0.000 & 0.000 & 0.005 \\
\hline & 272 & 0.000 & 0.000 & 0.000 & 0.000 & 0.008 & 0.000 & 0.000 & 0.000 & 0.000 & 0.000 & 0.000 & 0.000 & 0.000 & 0.000 & 0.005 \\
\hline & 275 & 0.006 & 0.005 & 0.000 & 0.006 & 0.000 & 0.011 & 0.015 & 0.000 & 0.000 & 0.000 & 0.010 & 0.000 & 0.056 & 0.000 & 0.005 \\
\hline & 278 & 0.017 & 0.000 & 0.000 & 0.011 & 0.000 & 0.005 & 0.015 & 0.011 & 0.021 & 0.010 & 0.000 & 0.000 & 0.000 & 0.000 & 0.005 \\
\hline & 281 & 0.034 & 0.027 & 0.063 & 0.011 & 0.015 & 0.005 & 0.038 & 0.021 & 0.010 & 0.021 & 0.010 & 0.000 & 0.000 & 0.010 & 0.010 \\
\hline & 284 & 0.028 & 0.016 & 0.038 & 0.022 & 0.015 & 0.022 & 0.069 & 0.043 & 0.042 & 0.021 & 0.021 & 0.044 & 0.000 & 0.021 & 0.026 \\
\hline & 287 & 0.039 & 0.016 & 0.025 & 0.011 & 0.015 & 0.016 & 0.015 & 0.053 & 0.010 & 0.000 & 0.010 & 0.015 & 0.000 & 0.000 & 0.005 \\
\hline & 290 & 0.022 & 0.016 & 0.063 & 0.017 & 0.062 & 0.027 & 0.062 & 0.032 & 0.063 & 0.010 & 0.021 & 0.000 & 0.056 & 0.010 & 0.052 \\
\hline & 293 & 0.051 & 0.032 & 0.088 & 0.034 & 0.031 & 0.022 & 0.069 & 0.085 & 0.063 & 0.052 & 0.010 & 0.088 & 0.000 & 0.052 & 0.052 \\
\hline & 296 & 0.045 & 0.048 & 0.050 & 0.062 & 0.062 & 0.060 & 0.046 & 0.053 & 0.042 & 0.063 & 0.052 & 0.015 & 0.056 & 0.052 & 0.078 \\
\hline & 299 & 0.079 & 0.108 & 0.038 & 0.062 & 0.069 & 0.038 & 0.062 & 0.064 & 0.021 & 0.104 & 0.115 & 0.044 & 0.056 & 0.094 & 0.063 \\
\hline & 302 & 0.056 & 0.097 & 0.050 & 0.101 & 0.054 & 0.130 & 0.031 & 0.043 & 0.083 & 0.094 & 0.073 & 0.103 & 0.111 & 0.083 & 0.094 \\
\hline & 305 & 0.056 & 0.054 & 0.038 & 0.079 & 0.069 & 0.103 & 0.077 & 0.106 & 0.063 & 0.052 & 0.115 & 0.074 & 0.000 & 0.083 & 0.089 \\
\hline
\end{tabular}


Supplementary Table 2 (cont.)

\begin{tabular}{|c|c|c|c|c|c|c|c|c|c|c|c|c|c|c|c|c|}
\hline Locus & Allele & RAN & LBI & JON & HAU & MER & TAU & WEL12 & WEL13 & MAR & TAS & GOL & FIO & STE12 & STE14 & СНА \\
\hline \multirow[t]{22}{*}{ Pnova_28 } & 308 & 0.062 & 0.038 & 0.063 & 0.096 & 0.046 & 0.054 & 0.069 & 0.138 & 0.104 & 0.115 & 0.052 & 0.088 & 0.167 & 0.073 & 0.057 \\
\hline & 311 & 0.056 & 0.081 & 0.013 & 0.062 & 0.046 & 0.065 & 0.077 & 0.053 & 0.073 & 0.073 & 0.031 & 0.044 & 0.111 & 0.073 & 0.068 \\
\hline & 314 & 0.056 & 0.038 & 0.063 & 0.017 & 0.038 & 0.065 & 0.062 & 0.021 & 0.083 & 0.021 & 0.104 & 0.044 & 0.000 & 0.063 & 0.104 \\
\hline & 317 & 0.051 & 0.054 & 0.038 & 0.034 & 0.015 & 0.049 & 0.046 & 0.011 & 0.073 & 0.104 & 0.031 & 0.029 & 0.000 & 0.042 & 0.057 \\
\hline & 320 & 0.034 & 0.038 & 0.025 & 0.045 & 0.038 & 0.027 & 0.046 & 0.000 & 0.021 & 0.042 & 0.031 & 0.118 & 0.000 & 0.021 & 0.016 \\
\hline & 323 & 0.034 & 0.027 & 0.063 & 0.017 & 0.054 & 0.011 & 0.023 & 0.032 & 0.031 & 0.000 & 0.063 & 0.044 & 0.000 & 0.042 & 0.031 \\
\hline & 326 & 0.022 & 0.022 & 0.013 & 0.011 & 0.038 & 0.016 & 0.015 & 0.032 & 0.031 & 0.042 & 0.031 & 0.103 & 0.056 & 0.052 & 0.026 \\
\hline & 329 & 0.022 & 0.016 & 0.038 & 0.045 & 0.038 & 0.027 & 0.023 & 0.021 & 0.021 & 0.021 & 0.052 & 0.029 & 0.056 & 0.042 & 0.016 \\
\hline & 332 & 0.028 & 0.022 & 0.038 & 0.039 & 0.015 & 0.027 & 0.031 & 0.021 & 0.042 & 0.031 & 0.021 & 0.015 & 0.056 & 0.042 & 0.016 \\
\hline & 335 & 0.017 & 0.022 & 0.000 & 0.011 & 0.023 & 0.027 & 0.031 & 0.021 & 0.021 & 0.031 & 0.010 & 0.000 & 0.000 & 0.010 & 0.010 \\
\hline & 338 & 0.028 & 0.022 & 0.025 & 0.034 & 0.031 & 0.005 & 0.015 & 0.032 & 0.010 & 0.010 & 0.021 & 0.000 & 0.056 & 0.021 & 0.016 \\
\hline & 341 & 0.000 & 0.022 & 0.025 & 0.028 & 0.023 & 0.016 & 0.008 & 0.021 & 0.000 & 0.000 & 0.000 & 0.000 & 0.000 & 0.010 & 0.005 \\
\hline & 344 & 0.022 & 0.005 & 0.013 & 0.028 & 0.031 & 0.038 & 0.015 & 0.011 & 0.000 & 0.000 & 0.052 & 0.015 & 0.000 & 0.010 & 0.000 \\
\hline & 347 & 0.006 & 0.032 & 0.025 & 0.028 & 0.015 & 0.011 & 0.015 & 0.000 & 0.010 & 0.000 & 0.010 & 0.000 & 0.000 & 0.000 & 0.005 \\
\hline & 350 & 0.039 & 0.027 & 0.038 & 0.022 & 0.031 & 0.022 & 0.008 & 0.000 & 0.021 & 0.000 & 0.010 & 0.015 & 0.000 & 0.000 & 0.000 \\
\hline & 353 & 0.022 & 0.016 & 0.000 & 0.006 & 0.023 & 0.038 & 0.000 & 0.000 & 0.000 & 0.000 & 0.010 & 0.000 & 0.000 & 0.000 & 0.000 \\
\hline & 356 & 0.022 & 0.016 & 0.013 & 0.011 & 0.031 & 0.022 & 0.000 & 0.000 & 0.000 & 0.000 & 0.010 & 0.000 & 0.000 & 0.000 & 0.005 \\
\hline & 359 & 0.006 & 0.011 & 0.000 & 0.017 & 0.000 & 0.000 & 0.000 & 0.000 & 0.010 & 0.010 & 0.000 & 0.000 & 0.000 & 0.000 & 0.000 \\
\hline & 362 & 0.000 & 0.011 & 0.000 & 0.000 & 0.008 & 0.011 & 0.000 & 0.000 & 0.000 & 0.000 & 0.000 & 0.000 & 0.000 & 0.000 & 0.005 \\
\hline & 365 & 0.000 & 0.005 & 0.000 & 0.017 & 0.015 & 0.000 & 0.000 & 0.011 & 0.000 & 0.000 & 0.000 & 0.000 & 0.000 & 0.000 & 0.000 \\
\hline & 368 & 0.000 & 0.005 & 0.013 & 0.006 & 0.000 & 0.000 & 0.000 & 0.000 & 0.000 & 0.000 & 0.000 & 0.000 & 0.000 & 0.000 & 0.000 \\
\hline & 377 & 0.000 & 0.005 & 0.000 & 0.000 & 0.000 & 0.000 & 0.000 & 0.000 & 0.000 & 0.000 & 0.000 & 0.000 & 0.000 & 0.000 & 0.000 \\
\hline \multirow[t]{5}{*}{ Pnova_32 } & 167 & 0.010 & 0.000 & 0.000 & 0.000 & 0.000 & 0.000 & 0.000 & 0.000 & 0.000 & 0.000 & 0.000 & 0.000 & 0.000 & 0.000 & 0.000 \\
\hline & 173 & 0.015 & 0.000 & 0.000 & 0.010 & 0.007 & 0.010 & 0.000 & 0.010 & 0.000 & 0.021 & 0.011 & 0.000 & 0.000 & 0.000 & 0.005 \\
\hline & 175 & 0.658 & 0.670 & 0.625 & 0.606 & 0.558 & 0.536 & 0.642 & 0.635 & 0.660 & 0.594 & 0.660 & 0.529 & 0.375 & 0.396 & 0.633 \\
\hline & 177 & 0.240 & 0.229 & 0.275 & 0.298 & 0.333 & 0.347 & 0.276 & 0.260 & 0.280 & 0.281 & 0.266 & 0.397 & 0.375 & 0.219 & 0.270 \\
\hline & 179 & 0.041 & 0.043 & 0.025 & 0.025 & 0.036 & 0.051 & 0.030 & 0.042 & 0.020 & 0.073 & 0.021 & 0.015 & 0.000 & 0.052 & 0.026 \\
\hline
\end{tabular}


Supplementary Table 2 (cont.)

\begin{tabular}{|c|c|c|c|c|c|c|c|c|c|c|c|c|c|c|c|c|}
\hline Locus & Allele & RAN & LBI & JON & HAU & MER & TAU & WEL12 & WEL13 & MAR & TAS & GOL & FIO & STE12 & STE14 & СНA \\
\hline \multirow[t]{3}{*}{ Pnova_32 } & 181 & 0.036 & 0.032 & 0.050 & 0.056 & 0.014 & 0.036 & 0.022 & 0.042 & 0.020 & 0.010 & 0.011 & 0.044 & 0.188 & 0.333 & 0.041 \\
\hline & 183 & 0.000 & 0.027 & 0.025 & 0.005 & 0.051 & 0.015 & 0.022 & 0.010 & 0.020 & 0.021 & 0.032 & 0.000 & 0.063 & 0.000 & 0.026 \\
\hline & 185 & 0.000 & 0.000 & 0.000 & 0.000 & 0.000 & 0.005 & 0.007 & 0.000 & 0.000 & 0.000 & 0.000 & 0.015 & 0.000 & 0.000 & 0.000 \\
\hline \multirow[t]{15}{*}{ Pnova_33 } & 149 & 0.005 & 0.000 & 0.000 & 0.000 & 0.000 & 0.000 & 0.000 & 0.000 & 0.000 & 0.000 & 0.000 & 0.000 & 0.000 & 0.000 & 0.000 \\
\hline & 154 & 0.000 & 0.000 & 0.000 & 0.000 & 0.000 & 0.000 & 0.000 & 0.000 & 0.000 & 0.000 & 0.000 & 0.000 & 0.000 & 0.010 & 0.000 \\
\hline & 159 & 0.000 & 0.000 & 0.000 & 0.000 & 0.008 & 0.011 & 0.000 & 0.000 & 0.000 & 0.000 & 0.000 & 0.015 & 0.000 & 0.000 & 0.000 \\
\hline & 164 & 0.095 & 0.070 & 0.063 & 0.052 & 0.061 & 0.084 & 0.053 & 0.063 & 0.130 & 0.052 & 0.048 & 0.045 & 0.167 & 0.052 & 0.082 \\
\hline & 169 & 0.211 & 0.279 & 0.213 & 0.222 & 0.159 & 0.216 & 0.250 & 0.250 & 0.190 & 0.333 & 0.310 & 0.212 & 0.222 & 0.260 & 0.296 \\
\hline & 174 & 0.295 & 0.297 & 0.350 & 0.330 & 0.402 & 0.289 & 0.326 & 0.250 & 0.300 & 0.292 & 0.298 & 0.424 & 0.222 & 0.260 & 0.281 \\
\hline & 179 & 0.211 & 0.192 & 0.263 & 0.196 & 0.242 & 0.263 & 0.182 & 0.219 & 0.150 & 0.167 & 0.155 & 0.273 & 0.167 & 0.260 & 0.173 \\
\hline & 184 & 0.126 & 0.058 & 0.038 & 0.113 & 0.053 & 0.053 & 0.076 & 0.125 & 0.120 & 0.083 & 0.083 & 0.000 & 0.111 & 0.094 & 0.071 \\
\hline & 189 & 0.021 & 0.058 & 0.075 & 0.036 & 0.015 & 0.047 & 0.076 & 0.073 & 0.020 & 0.031 & 0.060 & 0.030 & 0.056 & 0.031 & 0.020 \\
\hline & 194 & 0.005 & 0.017 & 0.000 & 0.010 & 0.008 & 0.005 & 0.015 & 0.010 & 0.010 & 0.021 & 0.048 & 0.000 & 0.000 & 0.010 & 0.026 \\
\hline & 199 & 0.021 & 0.012 & 0.000 & 0.010 & 0.023 & 0.016 & 0.000 & 0.010 & 0.030 & 0.010 & 0.000 & 0.000 & 0.000 & 0.000 & 0.015 \\
\hline & 204 & 0.000 & 0.012 & 0.000 & 0.021 & 0.008 & 0.016 & 0.015 & 0.000 & 0.010 & 0.000 & 0.000 & 0.000 & 0.056 & 0.021 & 0.015 \\
\hline & 209 & 0.011 & 0.006 & 0.000 & 0.005 & 0.000 & 0.000 & 0.000 & 0.000 & 0.040 & 0.010 & 0.000 & 0.000 & 0.000 & 0.000 & 0.020 \\
\hline & 214 & 0.000 & 0.000 & 0.000 & 0.005 & 0.015 & 0.000 & 0.008 & 0.000 & 0.000 & 0.000 & 0.000 & 0.000 & 0.000 & 0.000 & 0.000 \\
\hline & 229 & 0.000 & 0.000 & 0.000 & 0.000 & 0.008 & 0.000 & 0.000 & 0.000 & 0.000 & 0.000 & 0.000 & 0.000 & 0.000 & 0.000 & 0.000 \\
\hline
\end{tabular}


Supplementary Table 3 Allele frequencies for 10 microsatellite loci of Pecten novaezelandiae for 5 locations in the Coromandel fishery (Chapter 5 - spatial study).

\begin{tabular}{|c|c|c|c|c|c|c|}
\hline Locus & Allele & LBI & JON & HAU & MER & TAU \\
\hline \multirow[t]{8}{*}{ Pnova_01 } & 207 & 0.011 & 0.000 & 0.000 & 0.000 & 0.000 \\
\hline & 217 & 0.000 & 0.013 & 0.000 & 0.000 & 0.000 \\
\hline & 221 & 0.101 & 0.050 & 0.121 & 0.123 & 0.051 \\
\hline & 223 & 0.878 & 0.800 & 0.869 & 0.848 & 0.918 \\
\hline & 225 & 0.005 & 0.113 & 0.005 & 0.029 & 0.031 \\
\hline & 227 & 0.000 & 0.025 & 0.000 & 0.000 & 0.000 \\
\hline & 229 & 0.005 & 0.000 & 0.000 & 0.000 & 0.000 \\
\hline & 231 & 0.000 & 0.000 & 0.005 & 0.000 & 0.000 \\
\hline \multirow[t]{13}{*}{ Pnova_02 } & 161 & 0.000 & 0.000 & 0.010 & 0.007 & 0.000 \\
\hline & 163 & 0.000 & 0.013 & 0.000 & 0.000 & 0.010 \\
\hline & 165 & 0.021 & 0.013 & 0.025 & 0.036 & 0.035 \\
\hline & 167 & 0.266 & 0.295 & 0.197 & 0.239 & 0.288 \\
\hline & 169 & 0.479 & 0.500 & 0.556 & 0.522 & 0.510 \\
\hline & 171 & 0.069 & 0.090 & 0.045 & 0.094 & 0.045 \\
\hline & 173 & 0.128 & 0.000 & 0.101 & 0.065 & 0.051 \\
\hline & 175 & 0.016 & 0.064 & 0.035 & 0.014 & 0.030 \\
\hline & 177 & 0.011 & 0.026 & 0.005 & 0.014 & 0.010 \\
\hline & 179 & 0.011 & 0.000 & 0.010 & 0.007 & 0.010 \\
\hline & 181 & 0.000 & 0.000 & 0.010 & 0.000 & 0.005 \\
\hline & 183 & 0.000 & 0.000 & 0.005 & 0.000 & 0.000 \\
\hline & 185 & 0.000 & 0.000 & 0.000 & 0.000 & 0.005 \\
\hline \multirow[t]{9}{*}{ Pnova_04 } & 272 & 0.000 & 0.000 & 0.000 & 0.007 & 0.000 \\
\hline & 276 & 0.131 & 0.163 & 0.148 & 0.104 & 0.117 \\
\hline & 280 & 0.023 & 0.013 & 0.022 & 0.007 & 0.010 \\
\hline & 284 & 0.097 & 0.063 & 0.099 & 0.030 & 0.071 \\
\hline & 288 & 0.511 & 0.525 & 0.495 & 0.634 & 0.561 \\
\hline & 292 & 0.193 & 0.200 & 0.192 & 0.179 & 0.199 \\
\hline & 296 & 0.034 & 0.025 & 0.044 & 0.022 & 0.031 \\
\hline & 300 & 0.011 & 0.013 & 0.000 & 0.007 & 0.005 \\
\hline & 304 & 0.000 & 0.000 & 0.000 & 0.007 & 0.005 \\
\hline \multirow[t]{10}{*}{ Pnova_05 } & 131 & 0.043 & 0.033 & 0.052 & 0.059 & 0.041 \\
\hline & 135 & 0.161 & 0.117 & 0.063 & 0.096 & 0.112 \\
\hline & 139 & 0.140 & 0.167 & 0.092 & 0.154 & 0.168 \\
\hline & 143 & 0.403 & 0.233 & 0.385 & 0.397 & 0.434 \\
\hline & 147 & 0.167 & 0.300 & 0.293 & 0.213 & 0.163 \\
\hline & 151 & 0.054 & 0.067 & 0.075 & 0.051 & 0.046 \\
\hline & 155 & 0.016 & 0.033 & 0.011 & 0.029 & 0.015 \\
\hline & 159 & 0.005 & 0.000 & 0.011 & 0.000 & 0.000 \\
\hline & 163 & 0.011 & 0.050 & 0.000 & 0.000 & 0.010 \\
\hline & 167 & 0.000 & 0.000 & 0.017 & 0.000 & 0.005 \\
\hline
\end{tabular}


Supplementary Table 3 (Cont.)

\begin{tabular}{|c|c|c|c|c|c|c|}
\hline Locus & Allele & LBI & JON & HAU & MER & TAU \\
\hline Pnova_05 & 171 & 0.000 & 0.000 & 0.000 & 0.000 & 0.005 \\
\hline \multirow[t]{8}{*}{ Pnova_09 } & 112 & 0.043 & 0.013 & 0.046 & 0.030 & 0.026 \\
\hline & 116 & 0.033 & 0.038 & 0.056 & 0.075 & 0.052 \\
\hline & 120 & 0.766 & 0.800 & 0.724 & 0.679 & 0.742 \\
\hline & 124 & 0.054 & 0.063 & 0.066 & 0.090 & 0.052 \\
\hline & 128 & 0.087 & 0.063 & 0.082 & 0.082 & 0.098 \\
\hline & 132 & 0.011 & 0.025 & 0.026 & 0.030 & 0.015 \\
\hline & 136 & 0.000 & 0.000 & 0.000 & 0.007 & 0.010 \\
\hline & 144 & 0.005 & 0.000 & 0.000 & 0.007 & 0.005 \\
\hline \multirow[t]{12}{*}{ Pnova_10 } & 256 & 0.000 & 0.026 & 0.000 & 0.000 & 0.000 \\
\hline & 258 & 0.043 & 0.051 & 0.041 & 0.058 & 0.027 \\
\hline & 260 & 0.092 & 0.090 & 0.107 & 0.101 & 0.048 \\
\hline & 262 & 0.033 & 0.000 & 0.046 & 0.007 & 0.011 \\
\hline & 264 & 0.293 & 0.372 & 0.245 & 0.254 & 0.293 \\
\hline & 266 & 0.397 & 0.372 & 0.434 & 0.420 & 0.426 \\
\hline & 268 & 0.109 & 0.064 & 0.097 & 0.138 & 0.138 \\
\hline & 270 & 0.016 & 0.013 & 0.005 & 0.007 & 0.016 \\
\hline & 272 & 0.005 & 0.000 & 0.020 & 0.000 & 0.005 \\
\hline & 274 & 0.005 & 0.013 & 0.005 & 0.000 & 0.027 \\
\hline & 278 & 0.005 & 0.000 & 0.000 & 0.007 & 0.011 \\
\hline & 280 & 0.000 & 0.000 & 0.000 & 0.007 & 0.000 \\
\hline \multirow[t]{20}{*}{ Pnova_27 } & 151 & 0.011 & 0.000 & 0.005 & 0.000 & 0.000 \\
\hline & 167 & 0.000 & 0.000 & 0.000 & 0.008 & 0.000 \\
\hline & 171 & 0.000 & 0.000 & 0.011 & 0.000 & 0.000 \\
\hline & 183 & 0.000 & 0.000 & 0.005 & 0.000 & 0.000 \\
\hline & 187 & 0.005 & 0.000 & 0.005 & 0.000 & 0.000 \\
\hline & 191 & 0.011 & 0.028 & 0.016 & 0.000 & 0.011 \\
\hline & 195 & 0.005 & 0.028 & 0.005 & 0.008 & 0.005 \\
\hline & 199 & 0.021 & 0.014 & 0.026 & 0.030 & 0.027 \\
\hline & 203 & 0.005 & 0.014 & 0.021 & 0.061 & 0.011 \\
\hline & 207 & 0.032 & 0.014 & 0.032 & 0.023 & 0.049 \\
\hline & 211 & 0.016 & 0.014 & 0.047 & 0.015 & 0.038 \\
\hline & 215 & 0.032 & 0.042 & 0.021 & 0.015 & 0.049 \\
\hline & 219 & 0.069 & 0.042 & 0.026 & 0.030 & 0.055 \\
\hline & 223 & 0.053 & 0.014 & 0.032 & 0.061 & 0.066 \\
\hline & 227 & 0.048 & 0.111 & 0.053 & 0.030 & 0.060 \\
\hline & 231 & 0.064 & 0.042 & 0.053 & 0.061 & 0.049 \\
\hline & 235 & 0.064 & 0.042 & 0.047 & 0.053 & 0.044 \\
\hline & 239 & 0.059 & 0.042 & 0.037 & 0.053 & 0.071 \\
\hline & 243 & 0.059 & 0.097 & 0.042 & 0.053 & 0.077 \\
\hline & 247 & 0.064 & 0.069 & 0.079 & 0.023 & 0.071 \\
\hline
\end{tabular}


Supplementary Table 3 (Cont.)

\begin{tabular}{|c|c|c|c|c|c|c|}
\hline Locus & Allele & LBI & JON & HAU & MER & TAU \\
\hline \multirow[t]{20}{*}{ Pnova_27 } & 251 & 0.032 & 0.069 & 0.063 & 0.053 & 0.077 \\
\hline & 255 & 0.016 & 0.014 & 0.074 & 0.076 & 0.049 \\
\hline & 259 & 0.059 & 0.083 & 0.053 & 0.061 & 0.027 \\
\hline & 263 & 0.048 & 0.111 & 0.063 & 0.083 & 0.044 \\
\hline & 267 & 0.037 & 0.028 & 0.032 & 0.030 & 0.016 \\
\hline & 271 & 0.043 & 0.014 & 0.026 & 0.023 & 0.033 \\
\hline & 275 & 0.027 & 0.028 & 0.026 & 0.030 & 0.016 \\
\hline & 279 & 0.043 & 0.014 & 0.005 & 0.023 & 0.005 \\
\hline & 283 & 0.016 & 0.028 & 0.042 & 0.053 & 0.005 \\
\hline & 287 & 0.005 & 0.000 & 0.011 & 0.000 & 0.005 \\
\hline & 291 & 0.016 & 0.000 & 0.011 & 0.015 & 0.005 \\
\hline & 295 & 0.005 & 0.000 & 0.005 & 0.008 & 0.000 \\
\hline & 299 & 0.011 & 0.000 & 0.005 & 0.015 & 0.005 \\
\hline & 303 & 0.005 & 0.000 & 0.000 & 0.000 & 0.000 \\
\hline & 307 & 0.000 & 0.000 & 0.011 & 0.000 & 0.000 \\
\hline & 311 & 0.011 & 0.000 & 0.011 & 0.000 & 0.005 \\
\hline & 315 & 0.000 & 0.000 & 0.000 & 0.000 & 0.011 \\
\hline & 323 & 0.011 & 0.000 & 0.000 & 0.000 & 0.000 \\
\hline & 327 & 0.000 & 0.000 & 0.000 & 0.000 & 0.005 \\
\hline & 379 & 0.000 & 0.000 & 0.000 & 0.008 & 0.000 \\
\hline \multirow[t]{21}{*}{ Pnova_28 } & 248 & 0.000 & 0.000 & 0.000 & 0.000 & 0.005 \\
\hline & 254 & 0.022 & 0.025 & 0.011 & 0.031 & 0.022 \\
\hline & 257 & 0.000 & 0.013 & 0.000 & 0.000 & 0.000 \\
\hline & 266 & 0.005 & 0.013 & 0.000 & 0.008 & 0.000 \\
\hline & 269 & 0.022 & 0.000 & 0.000 & 0.000 & 0.000 \\
\hline & 272 & 0.000 & 0.000 & 0.000 & 0.008 & 0.000 \\
\hline & 275 & 0.005 & 0.000 & 0.006 & 0.000 & 0.011 \\
\hline & 278 & 0.000 & 0.000 & 0.011 & 0.000 & 0.005 \\
\hline & 281 & 0.027 & 0.063 & 0.011 & 0.015 & 0.005 \\
\hline & 284 & 0.016 & 0.038 & 0.022 & 0.015 & 0.022 \\
\hline & 287 & 0.016 & 0.025 & 0.011 & 0.015 & 0.016 \\
\hline & 290 & 0.016 & 0.063 & 0.017 & 0.062 & 0.027 \\
\hline & 293 & 0.032 & 0.088 & 0.034 & 0.031 & 0.022 \\
\hline & 296 & 0.048 & 0.050 & 0.062 & 0.062 & 0.060 \\
\hline & 299 & 0.108 & 0.038 & 0.062 & 0.069 & 0.038 \\
\hline & 302 & 0.097 & 0.050 & 0.101 & 0.054 & 0.130 \\
\hline & 305 & 0.054 & 0.038 & 0.079 & 0.069 & 0.103 \\
\hline & 308 & 0.038 & 0.063 & 0.096 & 0.046 & 0.054 \\
\hline & 311 & 0.081 & 0.013 & 0.062 & 0.046 & 0.065 \\
\hline & 314 & 0.038 & 0.063 & 0.017 & 0.038 & 0.065 \\
\hline & 317 & 0.054 & 0.038 & 0.034 & 0.015 & 0.049 \\
\hline
\end{tabular}


Supplementary Table 3 (Cont.)

\begin{tabular}{|c|c|c|c|c|c|c|}
\hline Locus & Allele & LBI & JON & HAU & MER & TAU \\
\hline \multirow[t]{18}{*}{ Pnova_28 } & 320 & 0.038 & 0.025 & 0.045 & 0.038 & 0.027 \\
\hline & 323 & 0.027 & 0.063 & 0.017 & 0.054 & 0.011 \\
\hline & 326 & 0.022 & 0.013 & 0.011 & 0.038 & 0.016 \\
\hline & 329 & 0.016 & 0.038 & 0.045 & 0.038 & 0.027 \\
\hline & 332 & 0.022 & 0.038 & 0.039 & 0.015 & 0.027 \\
\hline & 335 & 0.022 & 0.000 & 0.011 & 0.023 & 0.027 \\
\hline & 338 & 0.022 & 0.025 & 0.034 & 0.031 & 0.005 \\
\hline & 341 & 0.022 & 0.025 & 0.028 & 0.023 & 0.016 \\
\hline & 344 & 0.005 & 0.013 & 0.028 & 0.031 & 0.038 \\
\hline & 347 & 0.032 & 0.025 & 0.028 & 0.015 & 0.011 \\
\hline & 350 & 0.027 & 0.038 & 0.022 & 0.031 & 0.022 \\
\hline & 353 & 0.016 & 0.000 & 0.006 & 0.023 & 0.038 \\
\hline & 356 & 0.016 & 0.013 & 0.011 & 0.031 & 0.022 \\
\hline & 359 & 0.011 & 0.000 & 0.017 & 0.000 & 0.000 \\
\hline & 362 & 0.011 & 0.000 & 0.000 & 0.008 & 0.011 \\
\hline & 365 & 0.005 & 0.000 & 0.017 & 0.015 & 0.000 \\
\hline & 368 & 0.005 & 0.013 & 0.006 & 0.000 & 0.000 \\
\hline & 377 & 0.005 & 0.000 & 0.000 & 0.000 & 0.000 \\
\hline \multirow[t]{7}{*}{ Pnova_32 } & 173 & 0.000 & 0.000 & 0.010 & 0.007 & 0.010 \\
\hline & 175 & 0.670 & 0.625 & 0.606 & 0.558 & 0.536 \\
\hline & 177 & 0.229 & 0.275 & 0.298 & 0.333 & 0.347 \\
\hline & 179 & 0.043 & 0.025 & 0.025 & 0.036 & 0.051 \\
\hline & 181 & 0.032 & 0.050 & 0.056 & 0.014 & 0.036 \\
\hline & 183 & 0.027 & 0.025 & 0.005 & 0.051 & 0.015 \\
\hline & 185 & 0.000 & 0.000 & 0.000 & 0.000 & 0.005 \\
\hline \multirow[t]{13}{*}{ Pnova_33 } & 159 & 0.000 & 0.000 & 0.000 & 0.008 & 0.011 \\
\hline & 164 & 0.070 & 0.063 & 0.052 & 0.061 & 0.084 \\
\hline & 169 & 0.279 & 0.213 & 0.222 & 0.159 & 0.216 \\
\hline & 174 & 0.297 & 0.350 & 0.330 & 0.402 & 0.289 \\
\hline & 179 & 0.192 & 0.263 & 0.196 & 0.242 & 0.263 \\
\hline & 184 & 0.058 & 0.038 & 0.113 & 0.053 & 0.053 \\
\hline & 189 & 0.058 & 0.075 & 0.036 & 0.015 & 0.047 \\
\hline & 194 & 0.017 & 0.000 & 0.010 & 0.008 & 0.005 \\
\hline & 199 & 0.012 & 0.000 & 0.010 & 0.023 & 0.016 \\
\hline & 204 & 0.012 & 0.000 & 0.021 & 0.008 & 0.016 \\
\hline & 209 & 0.006 & 0.000 & 0.005 & 0.000 & 0.000 \\
\hline & 214 & 0.000 & 0.000 & 0.005 & 0.015 & 0.000 \\
\hline & 229 & 0.000 & 0.000 & 0.000 & 0.008 & 0.000 \\
\hline
\end{tabular}


Supplementary Table 4 Allele frequencies for 10 microsatellite loci of Pecten novaezelandiae for 6 locations in the Coromandel fishery (Chapter 5 - temporal study).

\begin{tabular}{|c|c|c|c|c|c|c|c|}
\hline Locus & Allele & LBI & LBI14 & MER & MER14 & TAU & TAU14 \\
\hline \multirow[t]{5}{*}{ Pnova_01 } & 219 & 0.000 & 0.008 & 0.000 & 0.000 & 0.000 & 0.000 \\
\hline & 221 & 0.095 & 0.056 & 0.133 & 0.102 & 0.057 & 0.154 \\
\hline & 223 & 0.897 & 0.913 & 0.842 & 0.864 & 0.929 & 0.831 \\
\hline & 225 & 0.000 & 0.024 & 0.025 & 0.034 & 0.014 & 0.015 \\
\hline & 229 & 0.008 & 0.000 & 0.000 & 0.000 & 0.000 & 0.000 \\
\hline \multirow[t]{13}{*}{ Pnova_02 } & 159 & 0.000 & 0.016 & 0.000 & 0.000 & 0.000 & 0.000 \\
\hline & 161 & 0.000 & 0.016 & 0.000 & 0.017 & 0.000 & 0.000 \\
\hline & 163 & 0.000 & 0.000 & 0.000 & 0.000 & 0.014 & 0.000 \\
\hline & 165 & 0.032 & 0.040 & 0.042 & 0.034 & 0.050 & 0.008 \\
\hline & 167 & 0.242 & 0.290 & 0.250 & 0.216 & 0.236 & 0.250 \\
\hline & 169 & 0.460 & 0.492 & 0.517 & 0.543 & 0.557 & 0.540 \\
\hline & 171 & 0.073 & 0.040 & 0.100 & 0.086 & 0.029 & 0.081 \\
\hline & 173 & 0.145 & 0.081 & 0.075 & 0.086 & 0.064 & 0.073 \\
\hline & 175 & 0.024 & 0.016 & 0.000 & 0.017 & 0.021 & 0.024 \\
\hline & 177 & 0.008 & 0.000 & 0.017 & 0.000 & 0.007 & 0.008 \\
\hline & 179 & 0.016 & 0.000 & 0.000 & 0.000 & 0.007 & 0.016 \\
\hline & 181 & 0.000 & 0.008 & 0.000 & 0.000 & 0.007 & 0.000 \\
\hline & 185 & 0.000 & 0.000 & 0.000 & 0.000 & 0.007 & 0.000 \\
\hline \multirow[t]{8}{*}{ Pnova_04 } & 276 & 0.129 & 0.197 & 0.103 & 0.167 & 0.101 & 0.103 \\
\hline & 280 & 0.026 & 0.016 & 0.009 & 0.018 & 0.014 & 0.000 \\
\hline & 284 & 0.069 & 0.090 & 0.034 & 0.088 & 0.065 & 0.071 \\
\hline & 288 & 0.552 & 0.451 & 0.638 & 0.561 & 0.558 & 0.540 \\
\hline & 292 & 0.198 & 0.230 & 0.172 & 0.140 & 0.210 & 0.214 \\
\hline & 296 & 0.026 & 0.008 & 0.026 & 0.026 & 0.036 & 0.056 \\
\hline & 300 & 0.000 & 0.008 & 0.009 & 0.000 & 0.007 & 0.016 \\
\hline & 304 & 0.000 & 0.000 & 0.009 & 0.000 & 0.007 & 0.000 \\
\hline \multirow[t]{10}{*}{ Pnova_05 } & 131 & 0.057 & 0.094 & 0.067 & 0.058 & 0.043 & 0.045 \\
\hline & 135 & 0.131 & 0.102 & 0.108 & 0.133 & 0.116 & 0.136 \\
\hline & 139 & 0.148 & 0.164 & 0.175 & 0.175 & 0.159 & 0.167 \\
\hline & 143 & 0.385 & 0.414 & 0.367 & 0.383 & 0.457 & 0.364 \\
\hline & 147 & 0.189 & 0.156 & 0.208 & 0.167 & 0.145 & 0.212 \\
\hline & 151 & 0.049 & 0.031 & 0.050 & 0.067 & 0.043 & 0.030 \\
\hline & 155 & 0.025 & 0.031 & 0.025 & 0.008 & 0.014 & 0.038 \\
\hline & 159 & 0.008 & 0.000 & 0.000 & 0.008 & 0.000 & 0.000 \\
\hline & 163 & 0.008 & 0.008 & 0.000 & 0.000 & 0.014 & 0.008 \\
\hline & 167 & 0.000 & 0.000 & 0.000 & 0.000 & 0.007 & 0.000 \\
\hline \multirow[t]{8}{*}{ Pnova_09 } & 112 & 0.065 & 0.063 & 0.034 & 0.061 & 0.022 & 0.023 \\
\hline & 116 & 0.032 & 0.031 & 0.060 & 0.044 & 0.065 & 0.054 \\
\hline & 120 & 0.734 & 0.773 & 0.690 & 0.798 & 0.739 & 0.792 \\
\hline & 124 & 0.048 & 0.039 & 0.095 & 0.009 & 0.051 & 0.062 \\
\hline & 128 & 0.105 & 0.078 & 0.078 & 0.079 & 0.094 & 0.062 \\
\hline & 132 & 0.008 & 0.008 & 0.026 & 0.009 & 0.022 & 0.000 \\
\hline & 136 & 0.000 & 0.008 & 0.009 & 0.000 & 0.007 & 0.008 \\
\hline & 144 & 0.008 & 0.000 & 0.009 & 0.000 & 0.000 & 0.000 \\
\hline
\end{tabular}


Supplementary Table 4 (Cont.)

\begin{tabular}{|c|c|c|c|c|c|c|c|}
\hline Locus & Allele & LBI & LBI14 & MER & MER14 & TAU & TAU14 \\
\hline \multirow[t]{12}{*}{ Pnova_10 } & 256 & 0.000 & 0.000 & 0.000 & 0.042 & 0.000 & 0.016 \\
\hline & 258 & 0.049 & 0.047 & 0.050 & 0.100 & 0.022 & 0.070 \\
\hline & 260 & 0.107 & 0.109 & 0.092 & 0.100 & 0.051 & 0.078 \\
\hline & 262 & 0.016 & 0.023 & 0.008 & 0.008 & 0.014 & 0.039 \\
\hline & 264 & 0.295 & 0.242 & 0.250 & 0.225 & 0.275 & 0.242 \\
\hline & 266 & 0.410 & 0.406 & 0.425 & 0.383 & 0.435 & 0.422 \\
\hline & 268 & 0.107 & 0.125 & 0.158 & 0.108 & 0.138 & 0.078 \\
\hline & 270 & 0.016 & 0.023 & 0.008 & 0.008 & 0.022 & 0.031 \\
\hline & 272 & 0.000 & 0.008 & 0.000 & 0.025 & 0.007 & 0.008 \\
\hline & 274 & 0.000 & 0.016 & 0.000 & 0.000 & 0.036 & 0.000 \\
\hline & 278 & 0.000 & 0.000 & 0.000 & 0.000 & 0.000 & 0.016 \\
\hline & 280 & 0.000 & 0.000 & 0.008 & 0.000 & 0.000 & 0.000 \\
\hline \multirow[t]{33}{*}{ Pnova_27 } & 151 & 0.016 & 0.000 & 0.000 & 0.000 & 0.000 & 0.000 \\
\hline & 167 & 0.000 & 0.000 & 0.009 & 0.000 & 0.000 & 0.000 \\
\hline & 191 & 0.016 & 0.008 & 0.000 & 0.000 & 0.016 & 0.000 \\
\hline & 195 & 0.008 & 0.033 & 0.009 & 0.035 & 0.008 & 0.016 \\
\hline & 199 & 0.016 & 0.025 & 0.026 & 0.018 & 0.016 & 0.023 \\
\hline & 203 & 0.008 & 0.057 & 0.044 & 0.018 & 0.016 & 0.023 \\
\hline & 207 & 0.008 & 0.033 & 0.026 & 0.053 & 0.047 & 0.008 \\
\hline & 211 & 0.016 & 0.016 & 0.009 & 0.044 & 0.047 & 0.047 \\
\hline & 215 & 0.032 & 0.025 & 0.018 & 0.018 & 0.063 & 0.047 \\
\hline & 219 & 0.089 & 0.025 & 0.035 & 0.018 & 0.008 & 0.086 \\
\hline & 223 & 0.032 & 0.057 & 0.061 & 0.053 & 0.086 & 0.094 \\
\hline & 227 & 0.048 & 0.041 & 0.035 & 0.088 & 0.047 & 0.055 \\
\hline & 231 & 0.048 & 0.107 & 0.061 & 0.070 & 0.070 & 0.055 \\
\hline & 235 & 0.073 & 0.082 & 0.061 & 0.070 & 0.047 & 0.047 \\
\hline & 239 & 0.048 & 0.049 & 0.044 & 0.026 & 0.070 & 0.094 \\
\hline & 243 & 0.056 & 0.066 & 0.061 & 0.088 & 0.086 & 0.055 \\
\hline & 247 & 0.056 & 0.066 & 0.018 & 0.009 & 0.063 & 0.094 \\
\hline & 251 & 0.040 & 0.033 & 0.053 & 0.105 & 0.102 & 0.039 \\
\hline & 255 & 0.016 & 0.033 & 0.079 & 0.044 & 0.047 & 0.039 \\
\hline & 259 & 0.089 & 0.057 & 0.061 & 0.053 & 0.023 & 0.055 \\
\hline & 263 & 0.056 & 0.041 & 0.079 & 0.018 & 0.047 & 0.008 \\
\hline & 267 & 0.040 & 0.025 & 0.035 & 0.044 & 0.023 & 0.023 \\
\hline & 271 & 0.032 & 0.016 & 0.018 & 0.026 & 0.016 & 0.031 \\
\hline & 275 & 0.016 & 0.000 & 0.035 & 0.035 & 0.016 & 0.016 \\
\hline & 279 & 0.040 & 0.025 & 0.018 & 0.018 & 0.000 & 0.008 \\
\hline & 283 & 0.016 & 0.008 & 0.053 & 0.009 & 0.008 & 0.016 \\
\hline & 287 & 0.008 & 0.025 & 0.000 & 0.000 & 0.000 & 0.008 \\
\hline & 291 & 0.016 & 0.016 & 0.018 & 0.009 & 0.000 & 0.000 \\
\hline & 295 & 0.008 & 0.008 & 0.009 & 0.009 & 0.000 & 0.000 \\
\hline & 299 & 0.016 & 0.000 & 0.018 & 0.009 & 0.008 & 0.008 \\
\hline & 303 & 0.008 & 0.008 & 0.000 & 0.000 & 0.000 & 0.000 \\
\hline & 307 & 0.000 & 0.000 & 0.000 & 0.009 & 0.000 & 0.000 \\
\hline & 311 & 0.016 & 0.000 & 0.000 & 0.000 & 0.008 & 0.000 \\
\hline
\end{tabular}


Supplementary Table 4 (Cont.)

\begin{tabular}{|c|c|c|c|c|c|c|c|}
\hline Locus & Allele & LBI & LBI14 & MER & MER14 & TAU & TAU14 \\
\hline \multirow[t]{4}{*}{ Pnova_27 } & 315 & 0.000 & 0.000 & 0.000 & 0.000 & 0.016 & 0.000 \\
\hline & 319 & 0.000 & 0.016 & 0.000 & 0.000 & 0.000 & 0.000 \\
\hline & 323 & 0.008 & 0.000 & 0.000 & 0.009 & 0.000 & 0.008 \\
\hline & 379 & 0.000 & 0.000 & 0.009 & 0.000 & 0.000 & 0.000 \\
\hline \multirow[t]{35}{*}{ Pnova_28 } & 254 & 0.025 & 0.056 & 0.036 & 0.025 & 0.016 & 0.062 \\
\hline & 266 & 0.008 & 0.000 & 0.009 & 0.000 & 0.000 & 0.000 \\
\hline & 269 & 0.033 & 0.000 & 0.000 & 0.000 & 0.000 & 0.000 \\
\hline & 272 & 0.000 & 0.000 & 0.009 & 0.000 & 0.000 & 0.000 \\
\hline & 275 & 0.000 & 0.000 & 0.000 & 0.017 & 0.016 & 0.000 \\
\hline & 278 & 0.000 & 0.024 & 0.000 & 0.000 & 0.008 & 0.031 \\
\hline & 281 & 0.041 & 0.016 & 0.009 & 0.017 & 0.000 & 0.031 \\
\hline & 284 & 0.025 & 0.024 & 0.018 & 0.025 & 0.008 & 0.031 \\
\hline & 287 & 0.008 & 0.024 & 0.018 & 0.025 & 0.016 & 0.038 \\
\hline & 290 & 0.008 & 0.016 & 0.054 & 0.025 & 0.032 & 0.038 \\
\hline & 293 & 0.033 & 0.008 & 0.027 & 0.033 & 0.032 & 0.069 \\
\hline & 296 & 0.049 & 0.048 & 0.054 & 0.083 & 0.056 & 0.023 \\
\hline & 299 & 0.131 & 0.048 & 0.080 & 0.075 & 0.040 & 0.023 \\
\hline & 302 & 0.098 & 0.089 & 0.045 & 0.117 & 0.143 & 0.092 \\
\hline & 305 & 0.057 & 0.105 & 0.071 & 0.075 & 0.119 & 0.092 \\
\hline & 308 & 0.041 & 0.056 & 0.036 & 0.083 & 0.056 & 0.062 \\
\hline & 311 & 0.082 & 0.065 & 0.045 & 0.017 & 0.071 & 0.015 \\
\hline & 314 & 0.049 & 0.048 & 0.045 & 0.042 & 0.079 & 0.031 \\
\hline & 317 & 0.049 & 0.032 & 0.018 & 0.017 & 0.056 & 0.031 \\
\hline & 320 & 0.033 & 0.056 & 0.036 & 0.042 & 0.040 & 0.031 \\
\hline & 323 & 0.016 & 0.048 & 0.045 & 0.025 & 0.008 & 0.038 \\
\hline & 326 & 0.008 & 0.040 & 0.036 & 0.025 & 0.024 & 0.031 \\
\hline & 329 & 0.008 & 0.016 & 0.045 & 0.025 & 0.008 & 0.046 \\
\hline & 332 & 0.025 & 0.040 & 0.018 & 0.033 & 0.032 & 0.015 \\
\hline & 335 & 0.008 & 0.000 & 0.027 & 0.008 & 0.008 & 0.023 \\
\hline & 338 & 0.033 & 0.032 & 0.027 & 0.025 & 0.008 & 0.008 \\
\hline & 341 & 0.033 & 0.000 & 0.027 & 0.008 & 0.024 & 0.015 \\
\hline & 344 & 0.008 & 0.016 & 0.036 & 0.017 & 0.032 & 0.015 \\
\hline & 347 & 0.033 & 0.032 & 0.018 & 0.025 & 0.016 & 0.038 \\
\hline & 350 & 0.025 & 0.016 & 0.036 & 0.042 & 0.008 & 0.023 \\
\hline & 353 & 0.000 & 0.008 & 0.018 & 0.008 & 0.024 & 0.008 \\
\hline & 356 & 0.008 & 0.016 & 0.036 & 0.025 & 0.016 & 0.023 \\
\hline & 359 & 0.008 & 0.000 & 0.000 & 0.017 & 0.000 & 0.008 \\
\hline & 362 & 0.016 & 0.008 & 0.009 & 0.000 & 0.008 & 0.008 \\
\hline & 365 & 0.000 & 0.008 & 0.018 & 0.000 & 0.000 & 0.000 \\
\hline \multirow[t]{5}{*}{ Pnova_32 } & 173 & 0.000 & 0.000 & 0.008 & 0.000 & 0.000 & 0.008 \\
\hline & 175 & 0.685 & 0.578 & 0.583 & 0.690 & 0.536 & 0.538 \\
\hline & 177 & 0.202 & 0.297 & 0.308 & 0.267 & 0.348 & 0.311 \\
\hline & 179 & 0.065 & 0.016 & 0.033 & 0.034 & 0.058 & 0.045 \\
\hline & 181 & 0.016 & 0.070 & 0.017 & 0.000 & 0.036 & 0.061 \\
\hline
\end{tabular}


Supplementary Table 4 (Cont.)

\begin{tabular}{lrrrrrrr}
\hline Locus & Allele & \multicolumn{1}{c}{ LBI } & LBI1 & \multicolumn{1}{l}{ MER } & \multicolumn{1}{l}{ MER14 } & \multicolumn{1}{l}{ TAU } & \multicolumn{1}{c}{ TAU14 } \\
\hline Pnova_32 & $\mathbf{1 8 3}$ & 0.032 & 0.039 & 0.050 & 0.009 & 0.014 & 0.038 \\
& $\mathbf{1 8 5}$ & 0.000 & 0.000 & 0.000 & 0.000 & 0.007 & 0.000 \\
\hline Pnova_33 & $\mathbf{1 5 9}$ & 0.000 & 0.000 & 0.009 & 0.009 & 0.000 & 0.000 \\
& $\mathbf{1 6 4}$ & 0.061 & 0.056 & 0.060 & 0.044 & 0.065 & 0.144 \\
& $\mathbf{1 6 9}$ & 0.298 & 0.306 & 0.164 & 0.404 & 0.225 & 0.303 \\
& $\mathbf{1 7 4}$ & 0.316 & 0.395 & 0.388 & 0.219 & 0.283 & 0.235 \\
& $\mathbf{1 7 9}$ & 0.193 & 0.137 & 0.241 & 0.254 & 0.275 & 0.235 \\
& $\mathbf{1 8 4}$ & 0.035 & 0.073 & 0.060 & 0.026 & 0.051 & 0.068 \\
& $\mathbf{1 8 9}$ & 0.053 & 0.032 & 0.017 & 0.035 & 0.058 & 0.008 \\
& $\mathbf{1 9 4}$ & 0.026 & 0.000 & 0.009 & 0.000 & 0.007 & 0.000 \\
& $\mathbf{1 9 9}$ & 0.000 & 0.000 & 0.026 & 0.000 & 0.022 & 0.000 \\
& $\mathbf{2 0 4}$ & 0.009 & 0.000 & 0.000 & 0.009 & 0.014 & 0.000 \\
& $\mathbf{2 0 9}$ & 0.009 & 0.000 & 0.000 & 0.000 & 0.000 & 0.008 \\
& $\mathbf{2 1 4}$ & 0.000 & 0.000 & 0.017 & 0.000 & 0.000 & 0.000 \\
& $\mathbf{2 2 9}$ & 0.000 & 0.000 & 0.009 & 0.000 & 0.000 & 0.000 \\
\hline
\end{tabular}

